\title{
Knowing in Medical Practice
}

Expertise, Imaging Technologies and Interdisciplinarity

Sophie van Baalen

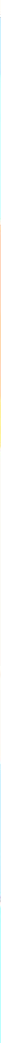





\section{KNOWING IN MEDICAL PRACTICE}

EXPERTISE, IMAGING TECHNOLOGIES AND INTERDISCIPLINARITY 



\title{
KNOWING IN MEDICAL PRACTICE
}

EXPERTISE, IMAGING TECHNOLOGIES AND INTERDISCIPLINARITY

\author{
PROEFSCHRIFT
}

ter verkrijging van

de graad van doctor aan de Universiteit Twente,

op gezag van de rector magnificus,

prof. dr. T.T.M. Palstra,

volgens besluit van het College voor Promoties

in het openbaar te verdedigen

op donderdag 31 januari 2019 om 14:45

door

Sophie Jacobine van Baalen

Geboren op 19 februari 1988

te Woerden, Nederland 
Dit proefschrift is goedgekeurd door:

de promotor:

prof. dr. ir. M. Boon

de co-promotoren:

dr. A. Carusi

prof. dr. ir. B. ten Haken

Cover design: Marilou Maes

Printed by: Ipskamp printing

Lay-out: Marilou Maes

ISBN: 978-90-365-4693-5

DOI: $10.3990 / 1.9789036546935$

(C) 2018 Sophie van Baalen, The Netherlands. All rights reserved. No parts of this thesis may be reproduced, stored in a retrieval system or transmitted in any form or by any means without permission of the author. Alle rechten voorbehouden. Niets uit deze uitgave mag worden vermenigvuldigd, in enige vorm of op enige wijze, zonder voorafgaande schriftelijke toestemming van de auteur. 


\section{Promotiecommissie}

\begin{tabular}{|c|c|c|}
\hline Voorzitter/secretaris & prof. dr. Th.A.J. Toonen & Universiteit Twente \\
\hline Promotor & prof. dr. ir. M. Boon & Universiteit Twente \\
\hline \multirow[t]{2}{*}{ Co-promotoren } & dr. A. Carusi. & Universiteit Twente \\
\hline & prof.dr.ir. B. ten Haken & Universiteit Twente \\
\hline Referee & dr. F. Russo & Universiteit van Amsterdam \\
\hline \multirow[t]{4}{*}{ Members } & prof. dr. ir. P.P.C.C. Verbeek & Universiteit Twente \\
\hline & dr. M. Boenink & Universiteit Twente \\
\hline & prof. dr. H.W. de Regt & Vrije Universiteit \\
\hline & prof. dr. I. Sabroe & University of Sheffield \\
\hline
\end{tabular}


This work is financed by an Aspasia grant (409.40216) of the Dutch National Science Foundation (NWO) for the project Philosophy of Science for the Engineering Sciences; by the work package Interdisciplinary Engineering Education at the 4TU-CEE (Centre for Engineering Education); by the Twente Graduate School (Bridging Grand and TGS Award 2014); and the Jo Kolk Studiefonds (external research at the University of Sheffield). The diffusion MRI research that was performed in this project was supported with a financial contribution from the UT Technical Medical Centre. 


\section{Acknowledgements}

This dissertation would not have been possible without my promotor, supervisor and mentor Mieke Boon. Dear Mieke, you have supported my ambition of writing a dissertation in which I would combine my background in technical medicine and my interest in philosophy of science in practice from well before I was close to graduating my masters. I am very grateful for the space you gave me to pursue my own interest while also always helping me make my work sharper, deeper and better. Thank you for having been there every step of the way, from first idea to the final revisions of this dissertation.

Others have made substantial contributions too, and I am grateful to them. Annamaria, thank you for a wonderful and very productive time in Sheffield. The empirical work we did and our discussions has truly been a catalyst for the development of the ideas I present in this thesis, and I have learned a lot from working with you. I also wish to thank the $\mathrm{PH}$-team, especially Ian and David, for being so hospitable and open to discussing the themes we were interested in.

Bennie, thanks for giving the opportunity to keep working in the diffusion-MRI field in parallel to my philosophical ambitions. I am also very grateful to all the colleagues and students who have worked with me on that project, especially Martijn for always taking the time to help me with scripts and technical questions, and Caroline and Marino for enabling the patient inclusion and their invaluable clinical input.

A very big thank you to my dear colleagues, for making work enjoyable most of the time, and supporting me when it was not. Many thanks to my friends and family for providing much-needed distractions from work. A special thanks to my parents for their unconditional support. I am so happy to be able to share and celebrate this with you.

Finally, to the two men in my life: Jori and Kasper. Working on this PhD was like a rollercoaster ride, but much longer. I am very grateful for the warm and happy home base the two of you provide, giving me confidence but also reminding me that there's more to life than a $\mathrm{PhD}$. I am very proud to have you.

Iedereen bedankt en heel veel liefs,

Sophie 


\section{Table of contents}

$\begin{array}{ll}\text { Introduction } & 13\end{array}$

$\begin{array}{ll}\text { Thesis outline } & 18\end{array}$

$\begin{array}{ll}\text { References } & 21\end{array}$

Chapter 1: An epistemological shift: from evidence-based medicine to 23

$\begin{array}{lr}\text { Abstract } & \text { epistemological responsibility. } \\ 1.1 . & 24\end{array}$

1.1. Introduction 25

1.2. Evidence-Based Medicine 26

1.3. 'Objective science' versus 'personal judgment' 30

1.4. Tensions between EBM and medical practice: a narrow view of science 32

1.5. Alternative Epistemologies 36

1.6. An alternative to EBM: the epistemological responsibility of doctors 43

$\begin{array}{ll}\text { References } & 47\end{array}$

Chapter 2: Evidence-Based Medicine versus Expertise: knowledge, skills and 51

Abstract $\quad 52$

2.1. Introduction 53

2.2. Epistemological responsibility in clinical practice $\quad 57$

$\begin{array}{ll}\text { 2.3. Medical expertise } & 61\end{array}$

2.4. Epistemic activities $\quad 63$

2.5. Tacit knowledge 65

2.6. The epistemological responsibility of experts in clinical decision-making $\quad 72$

References $\quad 75$

Chapter 3: Disciplinary perspectives and interdisciplinary research, a problem- $\quad 79$ oriented account $\quad 80$

$\begin{array}{lr}\text { Abstract } & 80\end{array}$

3.1. Introduction $\quad 81$

3.2. Disciplinary perspectives 83

3.3. Combining disciplinary perspectives 100

3.4. Interdisciplinary collaboration in developing medical technology for clinical practice 104

3.5. Disciplinary and interdisciplinary experts: The role of technical medicine 106

$\begin{array}{ll}\text { 3.6. Understanding interdisciplinarity } & 108\end{array}$

$\begin{array}{ll}\text { 3.7. Interdisciplinary expertise } & 113\end{array}$

3.8. Teaching interdisciplinarity - technical medicine 116

$\begin{array}{ll}\text { 3.9. Conclusion } & 119\end{array}$

$\begin{array}{ll}\text { References } & 121\end{array}$ 
4.1. Introduction $\quad 127$

4.2. Philosophical literature on imaging and scientific representation 131

4.3. Case study 138

4.4. Searching for evidence 142

4.5. Searching for clinical claims 152

4.6. Conclusion 162

$\begin{array}{ll}\text { References } & 166\end{array}$

Chapter 5: A social-technological epistemology of clinical decision-making as $\quad 169$

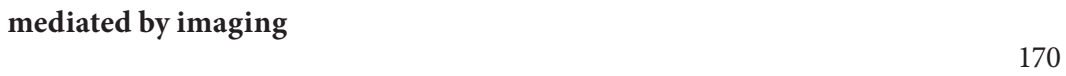

5.2. Methods 175

5.3. Results 176

$\begin{array}{ll}\text { 5.4. Discussion } & 187\end{array}$

$\begin{array}{ll}\text { References } & 190\end{array}$

Chapter 6: Implicit trust in clinical decision-making by multidisciplinary teams 195 Abstract 196

6.1. Introduction 197

6.2. Pulmonary hypertension 203

6.3. Implicit trust in a multidisciplinary team 206

6.4. Imaging mediates trust practices in clinical decision-making: developing 215 common ways of seeing

$\begin{array}{ll}\text { 6.5. Discussion } & 219\end{array}$

References 225

$\begin{array}{ll}\text { Discussion and conclusions } & 229\end{array}$

Expertise 232

Interdisciplinarity $\quad 232$

Imaging technologies $\quad 235$

$\begin{array}{ll}\text { Methodology } 239 & 235\end{array}$

$\begin{array}{ll}\text { Appendix } & 245\end{array}$

Summary 246

Samenvatting 254

List of publications $\quad 262$

Biography 263 


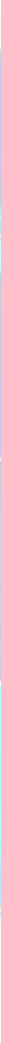




\section{Introduction}

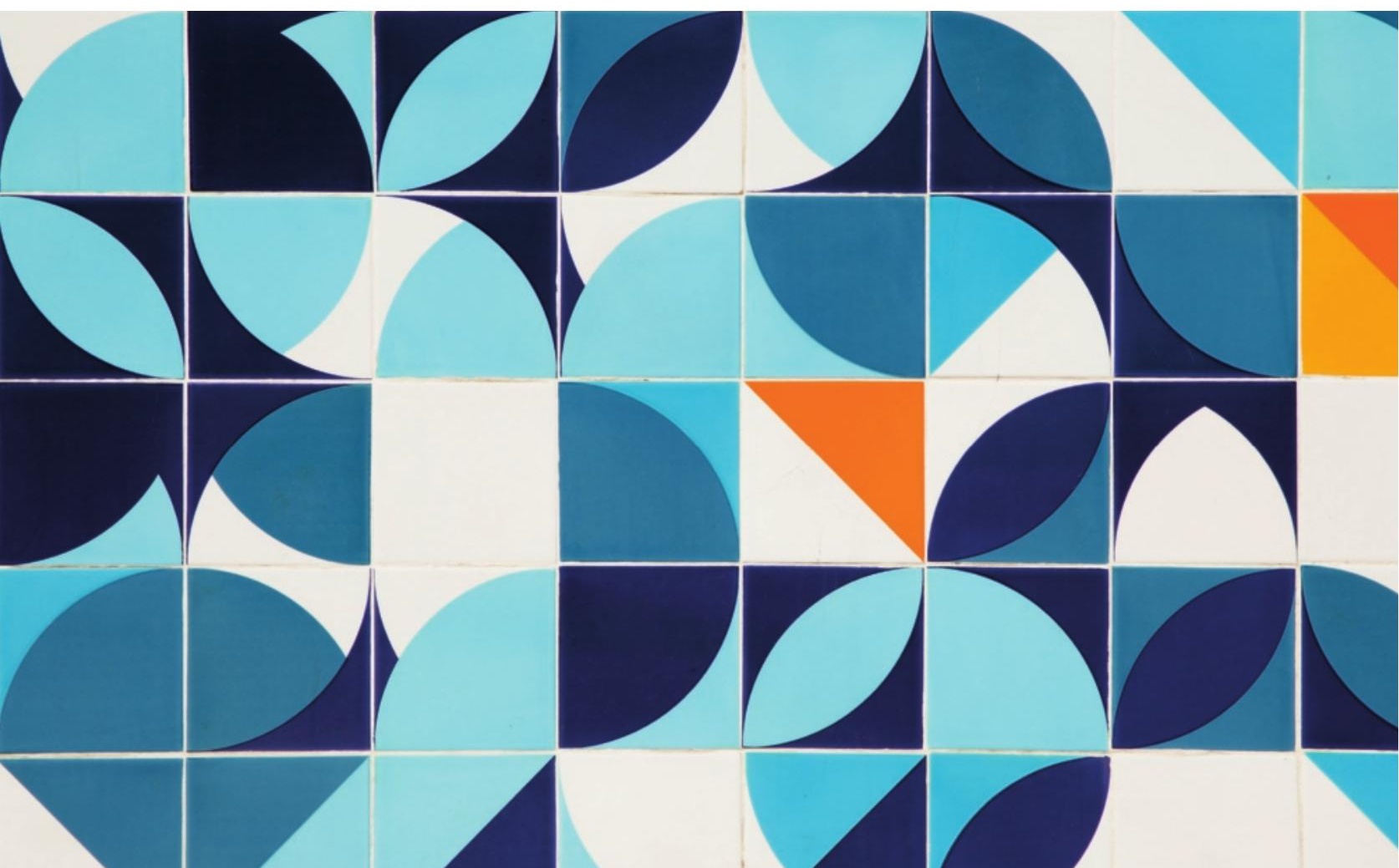


This dissertation is about knowing in medical practice. But, instead of a formal epistemological approach to knowledge, in which I would analyse whether knowledge is justified and applied following specific rules, this thesis revolves around the question, how exactly is knowledge $e^{1}$ constructed and used in medical practice? More specifically, I analyse the role of knowledge in medical decision-making concerning diagnosis and treatment; how knowledge is constructed in interdisciplinary collaborations; why and how knowledge is accepted; what knowledge is if not objective truth; what kind of expertise is needed to use and produce knowledge; what role social processes such as trust play in the construction and use of knowledge in multidisciplinary teams; how information that is provided by new imaging technologies is accepted as evidence to make diagnosis and treatment decisions; and the role that imaging technologies play in clinical decision-making. The main object of study for this dissertation is thus not theories or natural laws - the outcome of science - and how well they represent the real world (e.g., patients and their condition). Rather, I turn to the practice and the professionals who operate within this practice, their goals, their values and their cognitive abilities. In other words, in my analyses I have turned the focus from what is known to who is the knower and how does he or she come to know. For example, medical professionals construct knowledge - or as I will call it later in this dissertation a 'picture' or an 'epistemic tool' - of a patient for decision-making concerning diagnosis and treatment. The epistemological question that I ask is what people (e.g., scientists or medical professionals) $d o$ when they construct and use knowledge, and what criteria play a role in evaluating epistemic results. Therefore, I have analysed several real-life practices of clinical-decision-making and imaging technology development.

My interest in reasoning in medical practice, interdisciplinarity and the development and use of imaging technologies is a result of my experience as a student and researcher in Technical Medicine. During internships at clinical departments I talked with clinicians about their work, some of who were quite keen on entering in a philosophical discussion of their epistemic practices (e.g., regarding the epistemic activities related to the construction and use of knowledge in their professional practices). Based on these experiences, I have learned that current ideas about decision-making in clinical practice - i.e., the epistemology of evidence-based medicine (EBM) and the focus on individual knowers - are limited and because of that not always useful to evaluate and improve these practices. This has led me to study the conceptual underpinnings and history of EBM and perform a qualitative, empirical study of decision-making in actual clinical decision-making practices aiming

1 In this dissertation I use 'knowledge' as a loosely defined and broad concept, covering all kinds of results from epistemic processes, such as theories, concepts, natural laws, principles and models. 
to gain a well-informed conceptual understanding of how knowledge is generated and used in these practices. Additionally, I was interested in the epistemological aspects of developing and using imaging technology in clinical practice. Therefore, I have combined a focus on practice with concepts from the philosophy of science, by taking a philosophy of science in practice approach. The aim of the Society for Philosophy of Science in Practice (SPSP) is to develop an 'analytic framework that takes into consideration theory, practice and the world simultaneously,' by re-framing debates in traditional epistemology in terms of activities. ${ }^{2}$ Taking a philosophy of science in practice approach gave me the opportunity to develop an in-depth epistemological analysis of the activities involved in clinical decision-making, and the role of expertise, imaging technologies and interdisciplinarity in these practices. In addition, in the past four years I have worked as a researcher on the development of a new MRI tool for clinical practice together with engineers, radiologists, urologists and physicists (for a detailed description of this project, see Chapter 3). By being involved in this research I have myself encountered the epistemological issues and difficulties that researchers in this type of research face, for example issues related to interdisciplinary collaborations. I have analysed such issues using concepts from the philosophy of science, which I will introduce below. By introducing concepts from the philosophy of science, I have aimed to provide a vocabulary to knowers involved in (interdisciplinary) reasoning processes, in order for them to discuss, explain and justify their epistemic (i.e., related to knowledge construction or use) activities. Formal epistemology usually deals with knowledge abstracted away from the actual practices and seems to place heavy constraints on knowing and reasoning. This 'golden standard' for knowing and reasoning is quite impossible for knowers in practice to live up to, while at the same time accepting knowledge claims or decisions based on the 'authority' of experts is epistemically not adequate either. Therefore, I aim to find a middle way between the technical philosophical terms from formal epistemology and the imprecise and sometimes vague language practitioners themselves incline to use to describe their reasoning, using terms such as 'intuition' and 'the art of medicine' - which leaves a large part of the reasoning process implicit and seems to defer a lot of its justification to authority. The middle way that I aim to provide here requires leaving space for individual knowers to shape epistemic results according to their specific purposes and disciplinary perspectives while also enabling to open up reasoning processes for scrutiny and setting criteria to evaluate them.

The current 'golden standard' for knowing and reasoning in medicine - EBM - does not seem to suit the practice of medicine. The first question that I have asked is, in what way

2 See: http://www.philosophy-science-practice.org/about/mission-statement 
do doctors develop knowledge about their patients, and how can the quality of this process be warranted without placing unrealistic constraints or demands. To do this, I introduce the concept of epistemological responsibility, a concept that focuses on epistemic agents and underlines that the epistemological tasks of doctors - to gather, assess and integrate heterogeneous types of information and fit them into a 'picture' of a patient - involve a considerable amount of choice, deliberation and justification, for which they should be held accountable (Code 1984, 1987). To operate as an epistemologically responsible agent involves using and producing knowledge in a reliable way. Therefore, focusing on epistemological responsibility provides clinical decision-makers the flexibility to include all types of knowledge and use complex styles of reasoning while also demanding that certain epistemic criteria are met. To better understand these criteria, I use the concept of knowledge as epistemic tools (Boon and Knuuttila 2009; Boon 2017; Knuuttila and Boon 2011). Regarding the knowledge that a physician constructs of his patient as an epistemic tool that enables to execute further reasoning introduces other criteria than truthfulness. In addition to criteria such as internal consistency and coherency to available information, these criteria are related to the utility of the tool. The question that I then ask is, if physicians have epistemological responsibility, how can they meet this responsibility? For this, I turn to medical expertise, and ask what it entails. I introduce the idea that expertise is about what experts can do rather than (primarily) about what they know (Collins and Evans 2007). When it comes to knowledge construction, expertise involves the ability to perform epistemic activities (Chang 2012, 2014). Many authors consider tacit knowledge to be a crucial aspect of expertise, but see it as inarticulate and therefore closed off for reflection. In contrast to this, I return to Michael Polanyi's original notion of tacit knowledge to argue that knowledge is part of one's subsidiary or focal awareness, depending on the situation. Knowledge in one's subsidiary awareness can be reflected on and opened to scrutiny after an act of knowing (Polanyi 1958, 1966).

The question that comes up after introducing expertise is, how does one become an expert in a certain discipline? Philosophers such as Thomas Kuhn and Ludwig Fleck argue that experts are educated within a disciplinary community by which they internalize a 'disciplinary matrix' or 'thought style' (Fleck 1979; Kuhn 1970). I introduce the concept of disciplinary perspective to further analyse what this means. A disciplinary perspective is a coherent set of aspects such as goals, phenomena of interest, object of investigation, ways of modelling, theories and concepts, methodology, and instruments, practical constraints, epistemic values and scientific ideals that shapes what aspects of a target system disciplinary experts will 'see' or 'filter-out' and how they will approach research concerning the target system. This leads to the question, what does this notion of disciplinary expertise mean for interdisciplinary collaborations? I focus on problem-oriented 
interdisciplinary research (Schmidt 2011; Thorén and Persson 2013) to show that in cases were disciplinary experts collaborate to solve a problem concerning a certain target system - such as developing an imaging tool for clinical practice - it is not about integration of theories but about interaction between disciplines. In such collaborations, professionals from different disciplines each contribute to a solution that is internally consistent and coherent with each disciplinary perspective. This requires interdisciplinary expertise in addition to disciplinary expertise.

Concerning the development of a new imaging tool, I asked how a images gain meaning about the patient's condition in clinical decision-making. The assumption underlying current research and development practices is that innovation takes place in different stages, which starts with technology development and ends with adopting the technology into clinical practice, where engineers are mostly involved in the first stages and clinicians in that last stage. This practice does not always seem to be successful. In my view, one of the reasons that many innovative imaging tools do not reach practice is that clinicians (as future users) need to be involved in the development of imaging technologies from early on. By that I mean that clinicians have to make epistemic contributions to the development of the technology by clarifying what relevant information the images can provide, to make what clinical claims. Therefore, I have studied a multidisciplinary team involved in the development of a new imaging tool for clinical practice to illustrate that an interdisciplinary collaboration between engineers and clinicians is imperative to develop images that can provide information that is reliable, interpretable, useful and relevant for the specific context. I characterize the epistemic process by which a multidisciplinary team comes to a shared conclusion about what can be 'seen' in an image and what this 'tells' about a patient as interdisciplinary collaborative reasoning. In an interdisciplinary collaborative reasoning process team members enter into multiple cycles of abduction deduction - induction, in the sense of C.S. Peirce (1908).

Finally, I have studied decision-making in image assisted diagnosis and treatment of a complex disease called pulmonary hypertension $(\mathrm{PH})$ to understand how disciplinary professionals (i.e., pulmonary physicians, radiologists, nurses and pharmacologists) collaborate in clinical decision-making practice. Here, I introduce a socio-technological epistemology of clinical decision-making, as my qualitative study of this practice implied that clinical decision-making is distributed over professionals with different expertises and roles who collaborate to establish a shared decision concerning the diagnosis and treatment of their patients. Medical images are pervasive in medical practice, and play a key mediating role in three ways: first, as enablers or tools in acquiring information about patients; second, as communication facilitators between experts; and third, as framing the 
epistemic domain by setting standards by which novel imaging tools are assessed, serving as a reference point to which new tools are compared. The collaborative character of clinical decision-making leads to complex social practices of trust. I use the idea of implicit trust to characterise the type of trust that plays a role in these practices. Implicit trust is trust that is given without reasons in advance of a situation of epistemic dependence but instead is established within that situation. Another way that trust can be implicit is that it is contained or nested within other actions that are not directly about the epistemic dependability of others but about a task at hand - i.e., decision-making concerning diagnosis or treatment of a patient. In my qualitative study of this practice I aimed to uncover the 'mechanisms' by which trust operates. The processes of establishing reasons for trust are primarily related to accomplishing a task, and only indirectly about trusting someone else's expertise or competence. These processes establish a space of reasons (Carusi 2009) within which what it means to have reasons for trust, or not, gains a meaning and traction in these team-work settings. Based on the analyses I have sketched above, I will defend the following claims in this dissertation:

1. Doctors have epistemological responsibility for their clinical decisions, which means that they are responsible for the collection, critical appraisal, interpretation and fitting together of heterogeneous sources of evidence into a 'picture' of the patient, instead of deferring (a part of) their responsibility to clinical guidelines as is envisioned and allowed for by evidence-based medicine.

2. Understanding and evaluating medical expertise involves an account of the epistemic activities that clinicians should be able to perform and the cognitive skills that allow them to perform these activities.

3. Experts develop a disciplinary perspective that shapes how they deal with a target system. In interdisciplinary research aimed at problems in professional practices, multidisciplinary teams consisting of disciplinary experts interact around a problem, each exercising their own disciplinary perspective in dealing with aspects of the target system, rather than integrating theories.

4. For the development of a new imaging technology for clinical practice, the involvement of future users, the clinicians, is imperative. Technology developers and clinicians enter into a process of interdisciplinary collaborative reasoning to establish what is 'seen' in the image and what it can 'tell' about the patient's condition.

5. Knowing in current medical practice has an inherently social character - in the sense of knowledge being distributed over professionals with different expertises and roles, who collaborate to establish a shared decision. Medical imaging plays a mediating role in these practices. 
6. The collaborative character of epistemic practices in clinical decision-making leads to complex social practices of trust. Trust in these practices is implicit, in the sense that trusting the expertise of others occurs while the members of a team focus on other tasks, most importantly, building up a collection of appropriate reasons for accepting evidence that all team members share and have access to. This forms an intersubjective framework of common ways of identifying and assessing evidence. It is within these frameworks that trusting or mistrusting becomes meaningful.

Throughout the chapters of this dissertation, in which I defend the above claims, I develop two more general claims that bring out new ways of approaching epistemological questions and challenges in practice:

I. Knowers play an active and irreducible role in the construction and use of knowledge in practice.

II. Knowing in real-world practices has an inherently collaborative character.

\section{Thesis outline}

In Chapters 1 and 2, I will analyse clinical decision-making by referring to the central paradigm for constructing medical knowledge and using it in clinical decision-making, to wit, evidence-based medicine (EBM). In Chapter 1 I argue that the methodology preferred by EBM and the way EBM envisions the application of scientific results (rule-based reasoning following guidelines) does not accommodate clinical decision-making. Making justified decisions concerning diagnosis and treatment is more complex and refined than the rule-based reasoning that EBM theory promotes. It involves the gathering and assessment of many different types of information, qualitative and quantitative, general and particular, personal and detached, local and universal. Instead of dismissing professional judgment and expertise in medical decision-making as unscientific, I argue that doctors have a responsibility to gather and integrate relevant, yet heterogeneous information about each individual patient so as to construct a coherent 'picture' of the patient that enables further reasoning about the patient's condition. This aspect of their professional responsibility will be called epistemological responsibility.

In Chapter 2 I argue that a more detailed account of expertise will help to better understand the epistemological responsibility of doctors, in terms of how it can be developed and how it can be assessed. Therefore, I focus on epistemic tools and epistemic activities to analyse how knowledge and information are used in the reasoning processes to reach 
clinical decisions. Performing actions and using tools well requires skills that are developed as a part of a professional's expertise. I endorse a skill-based view of expertise, but add that an important aspect of expertise is the ability to perform epistemic activities related to medical reasoning at an expert level. As a consequence, expertise does not only involve epistemic content and physical skills, but also cognitive skills to perform epistemic activities. Gathering and critical assessment of relevant information, the construction of a coherent 'picture' of the individual patient from these heterogeneous pieces of information, and the application and adaptation of this 'picture' to the specific situation at hand are epistemic activities that clinicians should be able to perform skillfully. I will argue that cognitive skills and competence in epistemic activities are crucial aspects of medical expertise and doctors have a responsibility to develop, acquire and cultivate these skills and competence.

In Chapters 3 and 4 I turn to a different practice by presenting two cases involving the development of new medical imaging technologies, in which multiple experts from different disciplines are involved. In Chapter 3 I analyse interdisciplinary expertise by presenting a case of an interdisciplinary research project developing a new imaging tool for the characterisation of kidney tumours. I was involved in this project as principal investigator. Expertise is usually acquired within a certain discipline, and I argue that, as a result, experts have a disciplinary perspective that shapes what aspects of a target-system they will 'see' or 'filter-out' and how they will approach research concerning the target system. I view disciplinary perspectives as coherent sets of aspects such as goals, phenomena of interest, objects of investigation, ways of modelling, theories and concepts, methodology and instruments, practical constraints, epistemic values, and scientific ideals. An interdisciplinary solution is achieved by taking each perspective on the target system and using each of them to reason about a phenomenon of interest studied or dealt with from one disciplinary perspective (i.e., which problem concerning this phenomenon has to be addressed, and how, leading to what solution) and how that affects the phenomenon of interest studied by experts from another disciplinary perspective. Instead of integration of theories and concepts, I argue for a 'problem-oriented' account of interdisciplinarity, where disciplinary perspectives interact around a problem concerning a specific target system.

In Chapter $4 \mathrm{I}$ zoom in on actual reasoning processes in an interdisciplinary research project in order to point out why interactions between different experts during the development of the imaging technology are imperative for the successful development of a clinical imaging tool. Establishing how new images are interpreted and what can be inferred about the patient's condition based on the images results from an interdisciplinary collaborative reasoning process. It is through the interaction of the technology, 
the image, and the experts' input that the representation and interpretation of an image become established.

In Chapter 5 and 6 I return to examples of decision-making in clinical practice to argue that knowing in current medical practice has an inherently social character - in the sense of knowledge being distributed over professionals with different expertises and roles, who collaborate to establish a shared decision. In Chapter 1 and 2 I have taken the individual physicians as the central object of analysis, as do most authors in the literature on the epistemology of clinical decision-making. In Chapters 5 I will argue that rather than focusing on the individual doctor's reasoning and knowledge, it is more fruitful to think of clinical decision-making as a form of distributed knowing in which technologies play a key mediating role. In my account, decision-making is not performed by any individual but is instead performed by an assemblage of people and instruments in coordinated actions. I will analyze clinical decision-making within a medical expert team involved in diagnosis and treatment of patients with a severe and complex disease called pulmonary hypertension, in order to show how multidisciplinary teams involved in collaborative clinical decision-making function in practice and how their collaborations are mediated by medical images.

In Chapter 6 I show the extent to which trust plays a pivotal role in the collaborations between team members and how trust is mediated by the imaging technologies. In repeated interactions, medical teams develop a collection of appropriate reasons for accepting evidence that all team members share and have access to, building up an intersubjective framework within which claims and interpretations can be justified, and decisions can be arrived at and shared by others. Medical images play a mediating role in these complex trust practices by allowing to build up these intersubjective frameworks. Images fulfil this role in two ways, firstly as epistemic objects that can be distributed among all members of a team, as well as interpreted and discussed, thus facilitating communication and sharing of information in a specific way. In addition, the sharing of images and communicating through them produces shared vision, through which the team members come to see and perceive in a common way. This reinforces the intersubjective framework of common ways of identifying and assessing evidence within which implicit trust in medical decision-making teams operate.

Finally, in the concluding chapter, I will draw connections between themes that have emerged in the chapters, reflect on how they relate to each other and where different approaches from chapters might conflict with each other. For example, how epistemological responsibility of clinicians relates to implicit trust in medical teams. I end with an reflection on my methodology. 


\section{References}

Boon, M. (2017), 'An engineering paradigm in the biomedical sciences: Knowledge as epistemic tool', Progress in Biophysics and Molecular Biology, 129, 25-39 DOI: 10.1016/j.pbiomolbio.2017.04.001.

Boon, M. and Knuuttila, T. (2009), 'Models as epistemic tools in engineering sciences: a pragmatic approach', in A. Meijers and Dov M. Gabbay (eds.), Philosophy of Technology an Engineering Sciences (Handbook of the philosophy of science; North Holland: Elsevier Science \& Technol), 687-720 ISBN: 978-0-444-51667-1

Carusi, A. (2009), 'Implicit Trust in the Space of Reasons and Implications for Technology Design: A Response to Justine Pila', Social Epistemology, 23 (1), 25-43 DOI: 10.1080/02691720902741423.

Chang, H. (2012), Is Water H2O? - Evidence, Realism and Pluralism (Boston Studies in the Philosophy and History of Science; Dordrecht: Springer) ISBN: 978-94-007-3931-4.

Chang, H. (2014), 'Epistemic Activities and Systems of Practice: Units of Analysis in Philosophy of Science After the Practice Turn.', in L. Soler, et al. (eds.), Science After the Practice Turn in the Philosophy, History and Social Studies of Science. (London: Routledge), 67-79 ISBN: 978-0415722957

Code, L. (1984), 'Toward a 'Responsibilist' Epistemology', Philosophy and Phenomenological Research, 45 (1), 29-50 DOI: $10.2307 / 2107325$.

Code, L. (1987), Epistemic Responsibility (99: Published for Brown University Press by University Press of New England) 123-26 ISBN: 087451407X

Collins, H. and Evans, R. (2007), Rethinking Expertise (Chicago, London: The University of Chicago Press) ISBN: 978-0-226-11360-9.

Fleck, L. (1979), Genesis and development of a scientific fact (Chicago: University of Chicago Press) ISBN: 9780226253251.

Knuuttila, T. and Boon, M. (2011), 'How do models give us knowledge? The case of Carnot's ideal heat engine', European Journal for Philosophy of Science, 1 (3), 309-34 DOI: 10.1007/s13194-011-0029-3.

Kuhn, T.S. (1970), The Structure of Scientific Revolutions, Second Edition, Enlarged, ed. O. Neurath (International Encyclopedia of Unified Science; Chicago and London: The University of Chicago Press.) ISBN: 0-22645803-2.

Peirce, C.S. (2018), 'A Neglected argument for the reality of God', <http://en.wikisource.org/wiki/A_Neglected_ Argument_for_the_Reality_of_God $>$, accessed 24-0.

Polanyi, M (1958), Personal Knowledge: Towards a Post-Critical Philosophy (1974 edn.; Chicago and London: The University of Chicago Press) ISBN: 041515149X.

Polanyi, M (1966), The Tacit Dimension (Chicago and London: The University of Chicago Press) ISBN: 9780226672984.

Schmidt, J. C. (2011), 'What is a problem?: On problem-oriented interdisciplinarity', Poiesis \& Praxis, 7 (4), 249-74 DOI: 10.1007/s10202-011-0091-0.

Thorén, H. and Persson, J. (2013), 'The Philosophy of Interdisciplinarity: Sustainability Science and ProblemFeeding', Journal for General Philosophy of Science, 44 (2), 337-55 DOI: 10.1007/s10838-013-9233-5. 


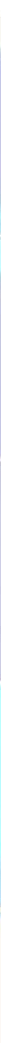




\section{An epistemological shift: \\ from evidence-based medicine to epistemological responsibility.}

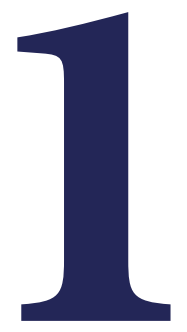

This chapter is adapted from: van Baalen, S. \& Boon, M. (2015). An epistemological shift: from evidence-based medicine to epistemological responsibility. J Eval Clin Pract, 21(3), 433-439. DOI: $10.1111 /$ jep.12282

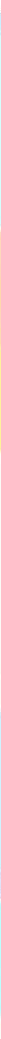




\begin{abstract}
Medicine is a field in which science and practice closely interact: doctors are expected to approach their work scientifically and to apply results from science in their everyday practice. However, its central epistemological ideal - evidence-based medicine (EBM) overlooks the complexities involved in the application of science in practice. EBM strives for objective and detached knowledge by promoting a single scientific methodology (comparative clinical studies) as the preferred source of knowledge and by formulating guidelines for clinical decision-making based on available evidence from RCTs. This epistemological ideal reflects the idea from traditional philosophy of science that application of science in practice merely involves deductive reasoning from general rules. By referring to contemporary philosophers of science and medicine, I argue that the use of scientific knowledge in practice requires knowledge from a variety of sources, ranging from general empirical or causal laws to very localised, qualitative and rich descriptions of the context, and knowledge of patient specificities, values and preferences. I present several alternative epistemologies of clinical decision-making to show that complex reasoning styles are involved in decision-making in practice and argue that the decision-maker (e.g., the medical doctor) plays a central role in the application of science to practice. Instead of striving for an objective and detached practice, it is, therefore, more fruitful to acknowledge the role of decision-makers and insist on their epistemological responsibility. Doctors have epistemological responsibility for their clinical decisions, being responsible for the collection, critical appraisal, interpretation and fitting together of heterogeneous sources of evidence into a 'picture' of the specific situation.
\end{abstract}




\subsection{Introduction}

Often, the application of science in practice is thought of as an uncomplicated and straightforward process. The outcome of science is general laws, and application involves deductive reasoning from these laws to the specific situation. Although this view has been contested by contemporary philosophers of science (for example, Nancy Cartwright, Steven Toulmin, Mieke Boon, and many others), and the biomedical sciences usually do not think in terms of laws but rather 'regularities,' it can still be recognized as the central ideal of the evidence-based medicine (EBM) movement. Therefore, EBM and the criticism that it has evoked can be regarded to be illustrative of the complexities that are involved in applying scientific results in a real-world context. My analysis of this specific situation will provide insights that are useful for the understanding of the application of science in other practical contexts as well.

In this chapter, I will argue that EBM does not serve the epistemic task of clinicians: to treat patients to the best of their abilities. To do this they need both particular information about the patient and also general scientific information about diseases and their prevention or treatment. So doctors have a professional responsibility to stay up-to-date with scientific developments in their field of work, to critically review the quality of all information they use, and to gather and integrate relevant information for clinical decision-making. In this chapter, I will take for granted the willingness of doctors to adopt this responsibility. My main focus is the epistemological difficulties of this responsibility in clinical decision-making.

I will argue that the epistemological ideal of the evidence-based medicine (EBM) paradigm currently favoured by health service providers, does not accommodate the epistemological challenges and responsibilities of doctors in their everyday clinical practice. In support of this argument, I will refer to several critiques of EBM, as well as arguments from the philosophy of science. Against the background of these critiques, I will propose that one of the problems with EBM in clinical practice is that its definition of 'scientific' is too narrow and that greater emphasis should be put on the epistemological responsibility of doctors. At the core of this shift is how knowledge about a specific situation, in this case, diagnosis and treatment of an individual patient, is realized. In the literature there seem to be two opposing ideas about medical epistemology in clinical decision-making. On the one hand, there are those who support the 'objective' or 'scientific' rule-based reasoning that underpins EBM, while on the other hand there are those who defend a more 'subjective' or 'personal' approach, often referred to as the 'art of medicine.' I evaluate several alternative medical epistemologies proposed in the literature, on which I build in 
developing an epistemology of high-quality clinical decision-making, which I consider more adequate than the normative ideal of scientifically supported, rule-based reasoning for guiding clinical practice as promoted by EBM. My alternative epistemology holds doctors accountable for epistemic considerations in clinical decision-making towards the diagnosis and treatment plan of individual patients, whereas EBM allows for deferring this part of their professional responsibility to strict clinical guidelines. I call this, 'epistemological responsibility,' by which I aim to grasp this alternative, more adequate ideal of clinical decision-making.

\subsection{Evidence-Based Medicine}

In current medical practice, like in other fields, the authority of doctors is no longer unquestionable: physicians cannot simply refer to their experience and authority when they make decisions, they are also expected to provide justification. In a response to the then-ubiquitous expert-opinion based medical practice, an anti-authoritarian ideal was formalized by the introduction of Evidence-based medicine (EBM) in 1992, when EBM was presented as a 'new paradigm for medical practice.' According to the evidence-based working group, the former paradigm was characterized by the idea that experience, clinical education, knowledge of pathophysiological mechanisms, and 'common sense' are sufficient for the evaluation of the efficacy of treatments and the value of diagnostic test. In their 'evidence-based' paradigm, these aspects are a 'crucial and necessary part' in medicine but both unsystematic observations and pathophysiological rationale can be misleading and are, therefore, 'insufficient grounds for clinical practice.' In the new paradigm, results from medical research, more specifically, controlled clinical studies, should be the basis for clinical decisions and physicians should be acquainted with the rules and skills required for critical appraisal of the literature (Guyatt et al. 1992). After criticism, mostly arguing that EBM promoted 'cookbook medicine' and could be misused by healthcare managers to make budget cuts, EBM was re-defined by Sackett et al. as "the conscientious, explicit, and judicious use of current best evidence in making decisions about the care of individual patients," (Sackett et al. 1996). The overall aim of EBM was to encourage the development of scientific methods such as clinical epidemiology and randomized controlled trials (RCTs) that could be used in the production of clinically applicable and unbiased data. Furthermore, EBM promoted the development and use of electronic databases that could be used to search the ever-increasing number of scientific medical publications. Alongside the databases and their associated search engines, methods were developed to guide doctors in their assessment of this mass of scientific literature. In the so-called 'hierarchy of evidence,' RCTs and systematic reviews of RCTs were presented as 


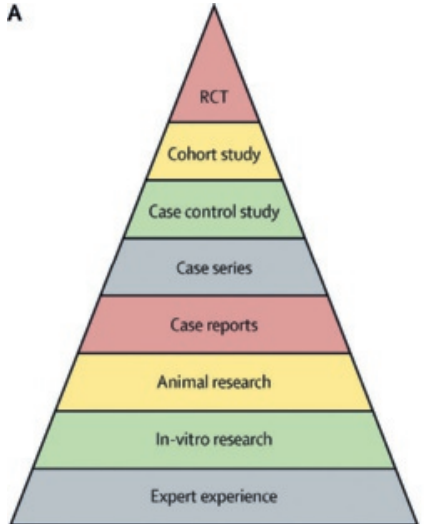

\begin{tabular}{|c|c|c|c|}
\hline Quality of evidence & Study design & Lower quality if* & Higher quality ift \\
\hline High & Randomised trial & \multirow{4}{*}{$\begin{array}{l}\text { Study limitations } \\
-1 \text { serious } \\
-2 \text { very serious } \\
\text { Inconsistency } \\
-1 \text { serious } \\
-2 \text { very serious } \\
\text { Indirectness } \\
-1 \text { serious } \\
-2 \text { very serious } \\
\text { Imprecision } \\
-1 \text { serious } \\
-2 \text { very serious } \\
\text { Publication bias } \\
-1 \text { likely } \\
-2 \text { very likely }\end{array}$} & \multirow{4}{*}{$\begin{array}{l}\text { Large effect } \\
+1 \text { large } \\
+2 \text { very large } \\
\text { Dose response } \\
+1 \text { evidence of a gradient } \\
\text { All plausible confounders } \\
+ \text { Would reduce a } \\
\text { demonstrated effect or } \\
+ \text { Would suggest a } \\
\text { spurious effect when } \\
\text { results show no effect }\end{array}$} \\
\hline Moderate & & & \\
\hline Low & Observational study & & \\
\hline Very low & & & \\
\hline
\end{tabular}

Figure 1.1: Hierarchy of evidence: traditional EBM versus GRADE (Djulbegovic and Guyatt 2017)3 Comparison of traditional EBM hierarchy of evidence (1991-2004) with GRADE classification of the quality of evidence (confidence, certainty; 2004 to present). (A) Traditional EBM hierarchy of evidence. (B) GRADE classification of the quality of evidence. EBM=evidence-based medicine. GRADE=Grading of Recommendations Assessment, Development, and Evaluation. RCT=randomised controlled trial. ${ }^{\star}$ Quality of study moves down one or two grades. †Quality of study moves up one or two grades

Despite the widespread use of the EBM paradigm, it is not without its critics. Several authors have expressed concerns about the translation of EBM research into clinical practice. Tonelli (1998) points out, for example, that there is an 'intrinsic gap' between the population-based research of EBM and its application to individual patients in the clinic. What applies in general to large groups does not necessarily apply to a specific person. And although EBM tries to minimize this gap by making differences between individuals explicit, this gap can never be closed completely since not all variations are quantifiable (Tonelli 1998). Along the same lines, Wyer and Silva (2009) argue that particular aspects of clinical decision-making, like "contextual impediments to implementation and considerations of patient values, patient preferences and experiences of disease" are poorly defined and integrated in EBM as a model for clinical practice (Wyer and Silva 2009, p.894). Therefore, according to Wyer and Silva, the weakness of EBM lies in its ambition to dictate clinical reasoning and action. Other authors, such as Worrall (2002), contest the status of RCTs as the only methodology for the production of scientific evidence. Worrall especially argues that the epistemic power of 'randomization' is continuously overestimated (Worrall 2002). Furthermore, Worrall says that some treatments cannot

3 Reprinted from The Lancet, 390 (10092), Djulbegovic B and Guyatt GH, Progress in evidence-based medicine: a quarter century on, 415-423., Copyright (2017), with permission from Elsevier. 
be assessed by RCTs because of moral or practical reasons. Finally, he argues that some often-used yet uncontested treatments like penicillin have not been tested in RCTs, which means that in actual clinical situations justification for treatments can result from other kinds of evidence than RCTs. In other words, Worrall believes that other types of evidence, like non-randomized studies, should not be dismissed or placed so low in the hierarchy of evidence for best clinical practice.

In response to criticisms about the rigidity of the hierarchy of evidence, proponents of EBM have developed a new system for evidence assessment, the Grading of Recommendations Assessment, Development and Evaluation (GRADE, see figure 1.1). The GRADE system allows for a more nuanced evaluation of clinical evidence, taking into account the quality of a study rather than the research method only (Vist et al. 2004). Still, the GRADE system only includes randomized trials and omits evidence obtained from pathophysiological mechanisms. Jeremy Howick (2011), who is generally in favour of the EBM view, argues that instead of completely dismissing the evidentiary role of mechanisms in favour of comparative clinical studies, mechanistic reasoning should be assigned a (slightly) more important role in EBM. Howick distinguishes between high- and low-quality mechanisms, where high-quality mechanisms should not be incomplete ${ }^{4}$ and the probabilistic and complex nature of biomedical mechanisms are taken into account when inferring claims about efficacy. According to Howick, high-quality mechanisms can add evidential weight, but are never necessary or sufficient to confirm the efficacy of a treatment or to solve the problem of 'external validity' of population research. However, Howick argues that comparative clinical studies have their shortcomings too, and therefore the strongest support for the efficacy of treatment combines evidence from high-quality mechanisms and evidence from high-quality comparative clinical studies. Moreover, Howick points out that "while the methodology of comparative clinical studies has been investigated for decades, standards for mechanistic reasoning have yet to be provided" (Howick 2011, p. 135). Recently, this project was taken up by the EBM+ network who "seek to improve the ways in which evidence-based medicine handles evidence of mechanisms." ${ }^{5}$ They argue that, although comparative clinical studies can establish causal relationships, mechanis-

4 With 'no incompleteness' Howick means to say that "there are no obvious gaps in knowledge of the inferential chain linking the intervention and the patient-relevant outcome." Whereas 'high quality mechanisms' for Howick means that "the probabilistic and complex nature of the mechanisms are explicitly taken into account when inferring from mechanisms to any claims that a particular intervention has a patient-relevant benefit," (Howick 2011, p. 144)

5 http://ebmplus.org/about/, visited 11-7-2018 
tic studies can play a role strengthening or weakening the evidence for this relationship. In addition, they present a method to assess mechanistic studies, evaluating the efficacy and external validity of causal claims and gathering and evaluating the evidence for the mechanism (Parkinnen et al. 2018). ${ }^{6}$

In short, the main problem with EBM identified by the authors cited above concerns the value of different types of medical research for clinical practice and the applicability of EBM research to clinical decision-making concerning diagnosis and treatment. From this, I conclude that EBM prefers particular methodology and knowledge that do not sufficiently accommodate clinical decision-making regarding individual patients. This is the consequence of doctors working with a specific goal: to improve the situation of individual patients. This goal conflicts with the general knowledge that EBM prioritises (basically produced in RCTs), and the methodology that EBM envisions for application of this generic knowledge in clinical practice (by formulating guidelines for diagnosis and treatment). EBM-based clinical guidelines prescribe clinical-decision making in an algorithmic manner. This apparent preference for rule-based reasoning conflicts with reasoning about individual patients, which requires integration of both general and particular information.

In 2014, advocates of the EBM movement claimed that the movement was in crisis due to a number of unintended consequences, among which the promotion of rule-based reasoning following clinical guidelines rather than careful deliberation and sound clinical judgment taking into account the localized and individual nature of medical practice (Greenhalgh et al. 2014). Timo Bolt and Frank Huisman (2015) characterize the current prominence of clinical guidelines in practice as a new form of authority-based practice that EBM originally aimed to move away from. They argue that although EBM has had an important role in eliminating bad medicine, in order to promote good medicine aspects such as clinical expertise, shared decision making and patient values should also be involved. In addition, Benjamin Djulbegovic and Gordon Guyatt, two of the EBM pioneers, argued that "the main challenge for EBM remains how to develop a coherent theory of decision making" and the right tools for clinicians to make point-of-care clinical decisions (Djulbegovic and Guyatt 2017).

It seems, therefore, that there are two important shortcomings with the epistemology for clinical decision-making defended by EBM: The hierarchy of evidence, which prefers

6 From overview on leaflet: http://ebmplus.org/wp-content/uploads/2017/12/leaflet.pdf, visited 16-7-2018 
RCTs over other methodologies, and the formulation of clinical guidelines that promote rule-based reasoning. These shortcomings are reflected in (traditional) philosophy of science, in which science is considered to produce one type of knowledge, that is, general laws, or regularities, and theories, while the application of science is often regarded to be a relatively straightforward process mainly involving deductive reasoning from the theories that are the product of science (e.g., Hempel 1965). EBM and the criticism that it has evoked are an illustration of the complexities that are involved in applying scientific results in a local, real-world context. It shows that when professionals use scientific knowledge to solve problems in practice, two issues arise. The first is that such epistemic products are not directly fit for use in real-world contexts: general laws have been derived from experiments in standardized or very specific situations and might not be applicable to the current practice. This is the problem of 'external validity' that Nancy Cartwright has put forward on the use of general laws or regularities in natural sciences (Cartwright 1999) and on the use of RCTs in social sciences and medicine (Cartwright 2011, 2012). The second is that, in order to use epistemic results in practice, professionals themselves need to have a specific set of abilities that cannot easily be captured in guidelines or general rules. These abilities involve knowledge and critical appraisal of products from research and science, as well as recognizing relevant particularities from the current context and bringing them together into a suitable problem definition and solution.

To address these epistemological problems, I will focus on the second issue - the nature of the abilities that are needed to apply epistemic results in (clinical) practice - by proposing a re-envisioning of best practice for clinical reasoning. According to EBM epistemology, the most effective way to come to a clinical decision is to use rule-based reasoning. I would argue that it is more appropriate to view clinical decision-making as a highly complex but still systematic epistemological process. To support this claim I will take a closer look at the epistemological difficulties inherent in EBM-based clinical decision-making, and I will also analyse the clinical reasoning behind 'best possible' diagnoses and treatments for individual patients. A fruitful starting-point for this discussion is the epistemological analysis of EBM by Michael Loughlin $(2008,2009)$, who also suggests an alternative approach (Loughlin 2008, 2009).

\section{3. 'Objective science' versus 'personal judgment'}

Loughlin's analysis evaluates EBM's philosophical claims about reasoning in medical practice and research. On the basis of this evaluation, Loughlin suggests that the idea of a hierarchy of evidence that prefers standardized trials (RCTs) over professional judgment is 
an impairment of the professional autonomy of physicians. According to Loughlin, EBM presupposes that objective decisions require impersonal mechanisms in order to rule out unwanted influences by emotions or self-interest. Therefore, in EBM-practice, objective reasoning is achieved by drawing general guidelines based on scientific research, which neglect the complexity of medical practice. Loughlin holds that in EBM, the demand for 'objective' and 'impersonal' decisions, as opposed to 'personal' or 'subjective,' results in professional autonomy being constrained by general guidelines.

According to Loughlin, EBM assumes a 'conceptual map' that dichotomizes concepts like 'objective' and 'rational' versus 'subjective' and 'personal.' He holds that this dichotomy between objective and subjective is a heritage of the logical positivist movement in the first half of the twentieth century. Logical positivism defines 'objectivity' and 'reality' in a very narrow sense: knowledge is grounded in observable facts and strict logical rules and these are required to draw general conclusions. It assumes that the only method to learn something about reality is through repeatable controlled experiments. By inference, this makes other methodologies and forms of knowledge 'biased'. In this light, the problem with EBM, according to Loughlin, is not its goal of working scientifically, but its reliance on a hierarchy of evidence that gives preference to RCTs as the 'gold standard' and so devaluates other forms of clinical proof.

The philosophical problem that underlies EBM, according to Loughlin, is that the (logical positivist) notion of 'science' assumed by EBM fails to acknowledge that the gathering, interpretation and application of evidence require human subjects making judgments. He believes that what is needed instead is "a robust defence of 'sound judgment' - not the pretence that knowledge can somehow be 'untainted' by the judgment of human subjects, but a rejection of the simplistic idea that all judgments are necessarily 'tainted"' (Loughlin 2008 p. 667). 'Sound judgments' require the judge to take into account several perspectives and weigh several options before coming to his or her own conclusion, and it is this that makes these kinds of judgments 'personal' and not 'objective' from an EBM point of view. But for Loughlin, judgments can be unbiased if they are not merely based on self-interest, emotions or opinion.

Loughlin continues his attempt to overcome the objective/subjective dichotomy by proposing an approach to medical epistemology that places personal judgments of professionals at the centre. According to Loughlin, this implies that, instead of prescribing standard guidelines for decision-making in diagnosis and treatment as in the epistemology of EBM, medical epistemology should assist practitioners in "developing their own rationally defensible conceptions of good practice and the intellectual basis of their activities," (Lough- 
lin 2009, p. 939). I agree with Loughlin's point that EBM assumes a view of 'scientific' that is too narrow, and with his explanation of how a (strict) dichotomy between subjective and objective leads to a dismissal of other valuable kinds of information. However, the concept of 'personal judgment' obfuscates the importance of studying relevant epistemological processes in a doctor's clinical reasoning. Calling a judgment 'personal' suggests that a physician's own consideration is the primary aspect of a judgment, and that what is considered, how this is considered and why a certain judgment is found the most appropriate, are secondary. In contrast, I will argue below that these latter aspects are primary in a physician's reasoning and determine the way he or she comes to a judgment that is sound.

The role of personal judgment and the insight that sound judgment does not necessarily follow the logic of EBM implies that other types of clinical reasoning can be acknowledged. A crucial additional aspect of this insight is that it requires doctors to be critically aware of the quality of their reasoning. Therefore, the concept of personal judgment should be extended to include the epistemological responsibility of doctors. The importance of introducing the concept of 'epistemological responsibility' to medical practice is to emphasize that doctors can be held accountable for how they make decisions concerning diagnosis and treatment.

\subsection{Tensions between EBM and medical practice: a narrow view of science}

One aspect of the tension between clinical practice and the presuppositions of EBM outlined above is the mismatch between knowledge provided by EBM methodologies (and formalized in guidelines) and the various kinds of knowledge used in clinical reasoning. From Kathryn Montgomery's analysis of clinical judgment and medical practice in How doctors think (Montgomery 2006) ${ }^{7}$ it is clear that they integrate many different kinds of information about a patient, such as, clinical observations, clinical measurements expressed in numbers, graphs or images (rather than clear-cut diagnoses), and the patient's account of his or her disease. Moreover, knowledge of (patho)physiological mechanisms plays an important role: it enables the physician to understand the relationships between these pieces of information, what other information is needed to make a sound judgment,

7 Montgomery's analysis of the intricate character of clinical judgements agrees with my own experience in clinical practices gained during a two-year clinical internships at several medical departments as a (former) student in technical medicine. 
to understand how symptoms relate to each other, and to understand how treatments cure diseases.

In other words, whereas EBM seeks to deliver epistemic products that represent the effects of treatments in a (statistically) truthful way, physicians seek knowledge that serves a specific epistemic purpose that not only focuses on 'justified knowledge,' but also takes into account the relevance, adequateness and usefulness (of different pieces of information) of knowledge for its local practical use, that is, to make diagnosis or treatment decisions concerning individual patients. This mismatch between the results from scientific research and the epistemic demands of the practices in which these results are meant to be applied is not unique to EBM and clinical practice. Similar issues have been raised for other sciences. According to Toulmin (2001), all sciences were, at least until the twentieth century, modelled after Newtonian/Euclid science, focusing on objective and detached observation and description in order to uncover the necessity and predictability of nature. Toulmin argues that no single way of theoretical reasoning should be placed above others. Rather, theories, as well as graphs, maps, physical models or computer graphics operate as models of phenomena that they represent. "If a representation pulls the phenomena together in a way that makes intelligible sense, giving a grasp of the field concerned, that is enough: the vivid image of airflow over a wing is both very memorable and entirely intelligible," (Toulmin 2001, p.173). According to Toulmin, there is not one explanatory representation that can serve all purposes; different models will have different results serving different practical needs. Similarly, in medicine, different ways of representing clinical knowledge (statistical, mechanistic, guidelines) serve as different kinds of input for different aspects of decision-making.

When considering the mismatch between EBM and clinical practice more closely, two questions arise. First, what is 'scientific'? According to Loughlin, the particular idea of science in the medical epistemology underlying EBM entails characteristic concepts of rationality and objectivity as opposites of personal, subjective and contextual. Second, why should medicine work scientifically? Doctors, patients, policy-makers and biomedical scientists regard medicine as a 'scientific endeavour,' its basic knowledge informed by scientific findings and methods, and its advancement enabled by technological inventions. I assume that the desire of medicine to work 'scientifically' derives from the confidence people have in scientific 'objectivity' and 'rationality', which is associated with an absence of personal interest, emotions or bias.

When addressing these two questions in order to understand how clinical practices produce good quality diagnosis and treatment, it appears that in a more appropriate account, 
the epistemology of these practices is interlinked with its ethics. Using the concept 'scientific' implies that the quality of knowledge is warranted, and that physicians base their decisions on detached knowledge rather than personal interest. However, as Loughlin shows, medical science and practice solely based on the concept of science in the narrow sense will not function satisfactorily, since the quality of diagnosis and treatment depends on a broader range of evidence than the evidence provided by RCTs and other methods of reasoning than the rule-based reasoning favoured in EBM. Furthermore, the scientific information produced by the approach preferred by EBM (RCTs) rarely provides all relevant knowledge that is crucial for a good quality diagnosis and treatment. Being 'scientific' in the narrow sense cannot warrant this quality. As a consequence, the ideal of attaining objectivity through rule-based reasoning, as promoted by the epistemology of EBM, cannot be maintained. Instead, following Toulmin, what I consider to be the central aspect of 'working scientifically' is systematic reasoning based on relevant information, one of the sources of which is scientific research but other sources are more local, contextual information that together make the situation intelligible.

Diagnosis and treatment involve gathering all relevant pieces of information, which, in turn, must be fitted together into a coherent 'picture' that best suits the specific situation of a patient. This 'picture' is not only a correct description of the patient's condition (i.e., a diagnosis that is either correct or incorrect) but also comprises a treatment plan and the expected effectivity based on the patient's specificities as well as general knowledge. In medical practice, doctors are held accountable both for the gathering of relevant information and for how they fit this information together. As has been argued, this does not only involve rule-based reasoning. While considering the available information, options are continually deduced and verified by doctors - this is because they understand, for instance, that one effect can have multiple causes and one cause can have multiple effects. Besides algorithmic, rule-based reasoning, 'creative' thinking and nuanced styles of reasoning are an inherent aspect of good clinical decision-making concerning diagnosis and treatment of a patient: the doctor aims to solve problems and to find compromises rather than strive for an 'objective truth.' Yet, the epistemology that underlies EBM dismisses these other ways of reasoning as subjective and less reliable. However, as I have claimed referring to Loughlin and Toulmin, both the sharp distinction between objective and subjective ways of reasoning and the disapproval of supposed subjective ways of reasoning enshrined in the epistemology of EBM are inappropriate for understanding the epistemology of actual clinical practice. Rather than being merely guided in their clinical reasoning by medical knowledge provided by RCTs and the preferred reasoning strategies outlined in the EBM guidelines, doctors carry responsibility for how they produce a diagnosis and treatment plan using more intricate reasoning methods. In order to account for this 
situation, I argue that a medical epistemology that takes into consideration the quality of the diagnosis and treatment plans necessarily needs to incorporate the responsibility of doctors. Below, I will give a more detailed description of this responsibility.

The adjusted hierarchy of evidence (GRADE) takes into account the quality of a study instead of just the method, and the EBM+ network has made an effort to find a role for evidence from mechanisms within the evidence-based epistemology. These recent developments in EBM are an important step towards a more diverse evidence-base in EBM. However, taking the quality of evidence into consideration also means a bigger involvement of the individual clinician who has to evaluate the evidence (according to criteria) and assess the applicability to the patient at hand. Moreover, EBM proponents have become aware of the complexities of clinical decision-making in practice and have expressed as the main challenge to progress as a movement that benefits patient outcome (Djulbegovic and Guyatt 2017; Greenhalgh et al. 2014; Howick 2011). However, the approaches they have proposed are still characterized by 'technoscientific reductionism' (Miles 2018). For example, EBM literature proposes that a method called 'decision analysis' should be used to include several components in a decision (Dowding and Thompson 2009). This method aims to provide guidance for the systematic combination of elements required for an evidence-based decision. Decisions are formalized by decision-trees, in which all possible outcomes of different options are identified. To weigh the options against each other, the probabilities of different outcomes are added along with patient values and preferences that are assigned a so-called 'utility', on a scale of $0-1$, and this is combined with probabilities to define the 'expected utility'. To identify the best option, the option with the highest expected utility is selected. Besides being laborious, the problem with this method is that it relies heavily on the availability of strong evidence, or, if strong evidence is not available, on educated guesswork. Clearly, this method aims to safeguard the objectivity of decision-making by proposing a more refined way of rule-based reasoning that relies on the objectivity of statistical reasoning. However, although this method combines the ideal of scientific objectivity with the need to include particular information about the patient, it does not accommodate the practice of clinical decision-making. Instead of focusing on the objectivity of a decision thereby rejecting all personal and particular aspects, an alternative medical epistemology should overcome this dichotomy. In developing this alternative epistemology, insights from other, richer, (medical) epistemologies will be utilized. 


\subsection{Alternative Epistemologies}

\subsubsection{Medicine as a natural and human science}

Hubert Dreyfus (2011) argues that "medicine is unique in being a combination of natural science and human science in which both are essential," assuming that there is an essential difference between natural and human science (Dreyfus 2011). In natural sciences, the studied object is first decontextualized and then objective measurements are performed. Based on these measurements, a theory about invisible objects is formed. In human sciences, this method is also attempted, but, according to Dreyfus, it is impossible to decontextualize humans, for the right interpretation, knowledge and understanding of the context is needed, which makes "objective" measurements impossible.

Dreyfus's analysis of medical practice is valuable because he shows that, to practice medicine, another approach besides that of "natural science" is needed. However, in my view, instead of emphasizing the supposedly essential difference between natural and human sciences, an epistemology that explains how contextual information and scientific knowledge are integrated will provide a better interpretation of medicine as a practice and as a science. I aim to grasp this by using the notion 'epistemological responsibility,' which describes the specific professional attitude and epistemological approach of clinical doctors who, rather than considering themselves followers of EBM rules and guidelines, consider themselves responsible for producing good quality diagnoses and treatments.

\subsubsection{The application of RCTs in practice}

EBM (implicitly) holds that correlations (or even causations) found in RCTs are true in the sense of generally applicable. This basic belief about scientific results (or the idea that scientific laws are universal) has been criticized for fundamental philosophical reasons by Nancy Cartwright (1999). She does not oppose the idea that laws are true but opposes the idea that they are true in every situation, regardless of the context. Instead, they are only true in the controlled environment (i.e., laboratory) that they were created in. The real challenge is to "to develop methodologies [...] for life in the messy world that we inevitably inhabit," (Cartwright 1999, p. 18).

In later work, Cartwright argues that extrapolating from RCTs or other (well performed) studies is not a good approach to make decisions about interventions in real-world contexts (Cartwright 2012). She shows that pilot studies or RCTs performed in one context are not sufficient to predict whether a policy will be effective or not in another context. According to Cartwright, RCTs can support the claim that an intervention 'works somewhere' but not predict that 'it will work here.' What is needed is to make sure that general 
laws, or regularities, are shared by both the study context and the target context. To do this, the relevant properties of the study context that support the outcome ('supporting factors') have to be identified and the presence of these properties in the target context has to be evaluated. These 'supporting factors' should be abstractions of the particular instance in the original context (e.g., from 'taking the medication at 8:00, 16:00 and 0:00' to 'taking the medication three times a day at regular intervals') and consequently translated into a particular instance in the target context (e.g., from 'taking the medication three times a day at regular intervals' to 'taking the pill at 6:00, 14:00 and 22:00'). Finally, users of generals laws need to ensure that causal factors interlock at both levels of abstraction (e.g., taking medication at regular intervals causes a stable drug level, which prevents the infection to return). According to Cartwright, these aspects require (additional) evidence of a different type than that RCTs provide, most importantly a great deal of local information on the study context and target context.

In short, Cartwright argues that the results from RCTs cannot be applied in practice in any straightforward manner but that it requires reasoning that is more dynamic and complex than the formal rule-based logic assumed by EBM epistemology and prescribed in clinical guidelines. She shows that for these results to give any information on whether an intervention might work in a specific situation involves identifying why an intervention works in a study population and predicting whether that also applies to the target population (or individual patient). According to Cartwright, this involves more than a simple 'compare and contrast.' It involves systematic reasoning to abstract away from particular instances to find those factors that have a share in the success of the intervention, and translate them to the context at hand. For clinicians, this means that they should not only identify whether the patient they are treating is similar to or different from the patients in the study population but also in what respect and predict whether that may affect the treatment. This requires a deeper understanding of the clinical study itself than simply knowing the outcome ('effective' or 'not effective'). A basic understanding of the mechanism with which the intervention operates and detailed information about the study population and the case at hand are all necessary. In my view, this also means that clinicians making these judgments have to be skilful at performing this type of systematic reasoning. They have to maintain a certain quality in identifying the relevant (local) information, finding the right level of abstractions, making correct inferences from theories, etc. All this is not accounted for by the kind of reasoning that is envisioned by EBM.

\subsubsection{The reflective practitioner}

EBM implicitly assumes that the only way to obtain scientific rigour in practice is by applying scientifically proven correlations in a standardized manner. As argued above, this 
position fails to account for the complexities involved with applying scientific results in practice. How can the reasoning that allows practitioners to deal with this in a systematic way be better understood? Donald Schön (1983) aims to provide an answer to this question. He analysed how practitioners in urban planning, social work and engineering deal with uncertainty, complexity and conflicting information and argues that practitioners have to find a balance between 'rigour and relevance' or between strictly following general rules prescribed by science (which he calls 'technical rationality') and doing something that is relevant to the current situation. Schön shows that practitioners seek out the particulars of a specific situation (rather than map it onto a standardized solution) and frame the situation to then explore the implications and unexpected consequences of this way of framing the situation. In the medical context, this could mean that the medical history of a patient is framed in terms of a particular diagnosis, and then there is an investigation of which other symptoms or test results would be expected if the patient did indeed have this disease, or what treatment would follow (and whether that fits the situation of the patient).

Epistemologically, this approach can be described as follows. In order to make sense of a unique situation, a practitioner sees the situation as another one from his or her repertoire of exemplars, from the whole of his or her prior experience. This 'seeing as' means seeing a situation as similar and at the same time different from another, while not yet being able to say in what respect - which can be determined through reflecting on seeing as. Schön gives an account of systematic reasoning in practices by arguing that practitioners perform 'on the spot experimentation,' with which he envisions a type of exploratory thought experiment. In their minds practitioners envision what it would be like if the hypothesis came true for a specific situation (e.g., if a building were placed in a certain environment) and then test the consequences (e.g., what would it mean for the internal and external structure of the building? How can the building be divided up in rooms? Will the building fit in with the surroundings? etc.) In this process, practitioners attempt to make the situation fit to a certain hypothesis but remain open to the possibility that it will not (e.g., the building in mind does not fit the environment). By reflecting on the consequences of a tested hypothesis and to a situation's resisting a certain framing, the practitioner can assess the suitability of a certain hypothesis. Schön calls this process reflection-in-action: "when someone reflects-in-action he becomes a researcher in the practice context. He is not dependent on the categories of established theory and technique but constructs a new theory of the unique case," (Schön 1983, p. 68). In short, Schön illustrates that, in practice, scientific knowledge is not directly applied but practitioners go beyond formal knowledge in a conscious and systematic process of going back and forth between the particular situation and his or her understanding of it. Schön's goal is to develop an "epistemology of practice which places technical problem solving within a 
broader context of reflective inquiry, shows how reflection-in-action may be rigorous in its own right." (Schön 1983, p.69)

\subsubsection{Case-based reasoning}

Rachel Ankeny (2010) in her analysis of the epistemic role of cases in medicine, shows that EBM is not the only relevant method for gaining and sharing knowledge in medical science (Ankeny 2010). Case reports are descriptions of individuals or small groups, which present us with 'surprising' or 'problematic' symptoms. Cases do not provide evidence in the same manner as RCTs, but, according to Ankeny, serve as vehicles for collecting facts and putting them in contact with each other. By studying a series of similar cases, researchers can prioritize certain facts and omit others. This, in the end, leads to the formulation of a working hypothesis, which can be confirmed by more 'conventional' research methods. Furthermore, a series of cases can provide knowledge of diagnosis, treatment and causations when RCTs or other conventional scientific methods are not possible for either ethical reasons (e.g., a disease, sign, or symptom cannot be induced deliberately for research purposes) or pragmatic reasons (e.g., a low frequency of a certain disease).

Ankeny (2014) argues that, although case-descriptions are based on uncontrolled observations (in contrast to controlled experiments, like RCTs) causal relationships can be derived from a series of cases (Ankeny 2014). In clinical practice, (a series of) case-descriptions from medical literature function as a source of evidence in the identification of the causes of the signs and symptoms observed in individual patients. In light of the goal of medicine, the clinical reasoning employed to find these causes is related to the instrumental nature of medicine, rather than a search for the objective truth. Clinical reasoning prefers identifying causes that are easy to remove and control in order to cure the patient or prevent disease. Therefore, the key to the determination of causes, according to Ankeny, is "what can be 'manipulated,' not as judged in principle but in practice," (Ankeny 2014, p.1008).

The use of cases in medical practice is an example of complex reasoning with a specific aim (finding a cause that can be intervened with to cure disease) using a different source of evidence than that from the RCTs promoted by EBM. Ankeny's analysis illustrates how in clinical practice, doctors use case reports from medical literature to gather (seemingly unrelated) facts, identify possible causes and consult experience from other practitioners with patients that present similar patterns of symptoms when diagnostic tools are not readily available. Ankeny illustrates that complex reasoning by means of cases presupposes that facts about individual patients can be projected onto other patients, which requires a rational process of systemizing, smoothing out the particulars of the individuals and refining the facts. This specific kind of reasoning is the work of experienced doctors and requires 
a specific set of skills that relate to what some would call the 'art of medicine,' which goes beyond the objective ruled-based reasoning of EBM epistemology. To ensure quality in such case-based reasoning in diagnosis and treatment, doctors must take responsibility for gathering relevant case reports from medical literature, drawing conclusions and translating the information to the situation of their specific patient. Hence, I argue that the responsible use of case-based reasoning should be considered a part of the epistemological responsibility of doctors, on which I will elaborate more in the Paragraph 1.6.

\subsubsection{Narrative reasoning}

In clinical practice, the patient's story is the starting point for identifying relevant facts of a particular case. Doctors usually ask their patients to describe the developments of signs and symptoms relating to the disease, putting these facts in a structure of time and space. An epistemology that focuses on reasoning based on these kinds of stories in medicine has been called 'narrative medicine.' Miriam Solomon (2008) analyses Rita Charon's Narrative Medicine (2008). Charon claims that techniques from the humanities are required to further develop and apply the technical achievements of medicine and that in this light narrative reasoning "constitutes a logic in its own right," distinguished from 'logico-scientific' reasoning. According to Solomon, Charon's work convincingly shows that narrative medicine can improve diagnosis and treatment because it yields more information, captures the uniqueness of a patient's situation, organizes coherence in otherwise disconnected facts, and allows for physicians to think creatively about and reflect upon their own practice. However, Solomon also warns that there can be 'narrative fallacy' when patients edit or omit certain aspects of their account in order to maximize coherence of the narrative or story.

Following up on the work of Charon, Solomon argues that the traditional epistemological dichotomy between medicine as a traditional science and the 'art of medicine' that encompasses the necessary skill of applying general knowledge to individual patients is 'not helpful.' To consider the intellectual skills often associated with 'the art of medicine' as 'non-scientific' is, according to Solomon, the result of a narrow sense of 'scientific' (according to Solomon, the hypothetico-deductive model). Solomon argues that narrative reasoning is a way to discover and hypothesize about causal connections, both in medicine and natural science. Making a good narrative is, according to Solomon, "an intellectual achievement that paves the way for more precise causal hypotheses" because "the requirements of narrative coherence and inclusion of all relevant facts put constraints on the stories that are told," (Solomon 2008, p. 416). Hence, the use of narrative is another complex reasoning method that can help doctors gather and fit together relevant information for diagnosis and treatment of a specific patient. This approach is very different from 
rule-based reasoning as it requires specific intellectual skills, and, as Charon suggests, even training in or knowledge of narrative theory from the humanities. The responsible use of narrative reasoning thus entails the epistemological skill to develop a coherent and complete narrative as part of constructing a coherent and consistent story about an individual patient's illness.

\subsubsection{Aesthetic judgments}

George Khushf (1999) argues that in addition to a scientific character, clinical judgement has an aesthetic character. He refers to Kant's aesthetic judgements, reflective judgements and determinative judgements to draw connections between the 'art' and 'science' in medicine. Khushf regards the diagnostic process as involving both determinative judgement (bringing a particular instance under a general concept) and reflective judgement (beginning with a particular and seeking out a concept). In the initial clinical encounter, a clinician develops a preliminary grasp of what is the matter with a patient (a possible set of diagnoses that integrate the particular signs and symptoms of the patient) in a reflective judgement, which he or she feels will benefit the aim of understanding (the diagnoses) which is then obtained through determinative judgment (establishing that the observed signs and symptoms fit with the diagnosis). Because reflective judgement draws on a broad knowledge base, this cannot be captured in the "cookbook format associated with guidelines and determinative judgment," (Khusfh 1999, p.149). According to Khushf, in order to understand how practitioners arrive at a preliminary understanding, or how they make reflective judgements "two aspects of understanding in medical practice must be addressed: 1) the current knowledge base, including medical theory and experience regarding its application; and 2) an awareness of the way determinative judgment proceeds, and the way it is nested within the broader practice of medicine," (ibid, p. 149). The merit of the reflective judgement ('diagnostic elegance') is assessed as a harmonisation between the preliminary idea and the demands of understanding that are placed on the preliminary idea by medical practice. These demands are rooted in the meta-scientific ideals of medicine, which enable the practice of medicine as a scientific practice (i.e., the diagnosis that follows from the preliminary grasp can be tested in a way that follows accepted epistemological standards in medicine).

According to Khushf, this harmonisation implies the feeling that the theory that a practitioner has developed for a specific situation (i.e., the diagnosis of a particular patient) and the practice in which that situation occurs fit together nicely according to the scientific ideal or epistemic standards of medicine. According to Khushf, this is an aesthetic judgment. "Kant associates this feeling accord between understanding (with its ends) and the particular, non-conceptualized manifold with aesthetic pleasure. Aesthetic judgment 
is made possible by spontaneous activity in which imagination and understanding are brought into harmony. [...] This harmony arouses a feeling of pleasure" (Khusfh 1999, p.148).

In short, Khushf shows that diagnosis is more than a simple 'categorization problem,' i.e., a matter of bringing a particular instance (a patient) under a universal (the disease). Rather, each patient requires the formation of a theory (diagnosis) that fits the particulars (signs and symptoms) of the patient at hand. Clinicians get a feeling of 'that's right' when their preliminary grasp of the situation, based on a range of information about the patient and their knowledge base, fits well together and that it can also be tested in a way that also fits in with the epistemic standards of medicine as a scientific practice. Striving for this feeling directs a physician's reasoning and gathering of information (i.e., initial patient history and physical exam) towards a coherent whole. This process is closely intertwined with clinicians' personal and collective (i.e., shared by the medical community) ideals concerning the goal of medicine as a scientific endeavour, directing a specific sense of rightness when formulating a diagnosis.

\subsubsection{Fitting together a 'picture' of a patient}

From the previous evaluation of alternative epistemologies, it follows that making justified decisions is more complex and refined than the rule-based reasoning that EBM theory promotes. It involves systematic reasoning towards adapting general knowledge to the specific situation (Cartwright), thought experiments to explore how a specific framing of the situation fits with that particular situation (Schön), bringing together heterogeneous information of a patient into a narrative (Solomon) or 'picture' of a patient that fits in nicely with all available information and epistemic standards (Khusfh). From this follows that doctors have a responsibility to 1) gather and use relevant information and knowledge, 2) employ different types of reasoning for specific situations, and 3) make the intellectual effort to use these different sources of knowledge and types of reasoning so as to come up with good diagnoses and treatment plans that fit with current perceptions of best practice. I believe that this is an important aspect of the professional responsibility of doctors that involves a certain professional attitude and epistemological ability, which I call epistemological responsibility. 


\subsection{An alternative to EBM: the epistemological responsibility of doctors}

Part of the professional responsibility of medical doctors consists of the responsibility to come up with the best possible diagnosis and treatment plan for individual patients. This responsibility involves epistemic activities that generate knowledge about the disease of a particular patient (the diagnosis) and about possible treatments. Therefore, the responsibility and epistemological challenges of doctors involves that they have to gather and integrate different sources of information - such as scientific-medical knowledge on diseases and treatments, diagnostic data on the patient, but also contextual information (e.g., particularities of the patient, availability of specific medical expertise, and constraints of the medical system). In other words, their responsibility and epistemological challenge are gathering and integrating relevant, yet heterogeneous aspects so as to construct a coherent picture of the patient's disease and possible treatment. A 'picture' of a patient cannot be constructed from heterogeneous sources using rule-based reasoning, as suggested in EBM epistemology. It requires complex other kinds of reasoning such as case-based and narrative reasoning, as well as professional judgments. Therefore, I propose that doctors should consider themselves epistemologically responsible for producing good quality diagnosis and treatment, instead of deferring their responsibility (and with that accountability) to strict clinical guidelines.

Lorraine Code (1984) introduces the concept of epistemic responsibility as a "potential new focal point for theory of knowledge," (Code 1984, p. 29). Code draws analogies between ethical and epistemological reasoning processes to show how an epistemological inquiry can be approached by a study of intellectual virtues, instead of searching for foundations as in traditional foundationalism. Important for my argument is Code's insight that, first, cognitive agents (such as doctors) have an important degree of choice when it comes to reasoning, and second, they are accountable for these choices. ${ }^{8}$ Therefore, in contrast to passive information-processers that are at best reliable, these agents should be evaluated in terms of responsibility. Following Code, I argue that the epistemological tasks of doctors involve a considerable amount of choice, deliberation and justification, for which they are held responsible in my account of medical decision-making. The notion

Code argues that this epistemology would parallel an ethics based upon moral virtues, and hence investigates which intellectual virtues an epistemologically responsible agent should possess. In the next chapter, in order to connect to theories of expertise, I will focus on skills rather than virtues, but still similar to Code, emphasize the importance of cognitive skills. 
of the epistemological responsibility of doctors as an alternative to the EBM epistemology, therefore, aims to grasp, on the one hand, the epistemological challenges of clinical decision-making, and the non-trivial responsibilities that doctors have concerning the epistemic activities in diagnosis and treatment, on the other. I will argue that this notion better suits the professional ideal of doctors in a clinical practice than the normative picture of EBM reasoning. Instead of emphasizing the importance of what is known, as in the epistemological ideal of EBM, the notion of epistemological responsibility focuses on the knowers and their epistemic location in time, place and other epistemologically relevant circumstances (Code 1987).

The epistemological tasks of doctors involve a considerable amount of choice and deliberation, for which they are held responsible. This starts with the gathering and assessment of relevant information. Furthermore, in contrast to EBM epistemology, I have argued that doctors are held responsible for the way in which they construct a coherent 'picture' of a patient from heterogeneous pieces of information. In fitting the different pieces of information together, these pieces need to be mutually adjusted, which requires epistemological choices. Finally, clinicians are held epistemologically responsible for applying and adapting this 'picture' in the process of dealing with individual patients.

The difficulties of these epistemological tasks are insufficiently recognized in current ideas on how to meet scientific and professional standards, both by EBM as a model for clinical decision-making, as well as by traditional philosophy of science. Firstly, EBM, although recognizing that local information must be integrated when applying clinical guidelines, does not address how this should be done, thereby suggesting that knowledge-formation about individual patients involves a more or less straightforward algorithmic way of reasoning. Secondly, scientific and objectified approaches such as EBM involve a narrow view of science that may not suit medical practices. This view suggests that the aim of a scientific approach in clinical decision-making is to find the objective 'truth' about the patient and that the method of EBM is the best possible method to reach this truth. However, I propose that the epistemological aim of scientific and objective approaches in clinical practice is not primarily to find objective 'truth,' but instead, to generate a 'picture' of individual patients, that is used for further reasoning about the diagnosis and treatment of the patient. I have argued that generating this picture usually involves complex kinds of reasoning as well as the rule-based reasoning proposed by EBM. As a consequence, I believe that the epistemological challenge for which doctors bear responsibility is how they build-up the 'picture' of their patient. 
So, whereas scientific approaches such as EBM aim at 'true descriptions,' I propose that epistemic results of doctors (e.g., the coherent 'picture' a doctor constructs of a specific patient, based on heterogeneous sources of information) must be considered in terms of their epistemic use (Boon and Knuuttila 2009; Knuuttila and Boon 2011). The objectivity and quality of these epistemic products are warranted by additional epistemic criteria - not only logical consistency and coherency with relevant knowledge (including knowledge generated in RCTs), but also pragmatic criteria that warrant the utility for this specific situation: to make the best possible diagnosis and treatment decisions for the individual patient, and for the specific situation. For example, if a doctor has more or better experience with one treatment for a certain case, whereas the results of RCTs suggest another, the treatment favoured in the RCTs may not be the most appropriate. In that case, doctors can argue to opt for a different choice of treatment, for example by referring to their personal success rates with that specific treatment or specificities of the case that makes that treatment more appropriate than another. Therefore, contextual and personal information, like the availability of a certain device in a hospital, a doctor's experience, and a patient's preferences are all relevant because they impact on what is the best decision in a specific situation.

What is scientific and why should medical practice work scientifically? By shifting the focus from 'objective truth' to 'epistemic use,' thus introducing alternative scientific criteria for guiding and assessing clinical reasoning, the strict dichotomy between subjective and objective resulting from the narrow view of science can be overcome. This alternative idea about the quality of clinical reasoning is covered by the notion 'epistemological responsibility.' This notion seems to fit well with the clinical context in which doctors are both epistemically challenged and inherently bound to a specific situation. A crucial aspect of this epistemological responsibility is that knowers bear a significant degree of responsibility in deciding what and how to (cognitively) process heterogeneous information into a coherent 'picture' of individual patients.

Finally, the analysis of reasoning processes and the application of science in clinical practice can be regarded as an interesting case for the philosophy of science in practice. The attempts of EBM to formulate simple rules to apply science in practice and the issues and complexities that have been raised in response are instructive for the study of the application of science in other practices. What can be taken away from this analysis for other contexts is that 'evidence-based' decision making, medical and other practices, requires a variety of types of evidence, from general empirical or causal laws to very localised, qualitative and rich descriptions. What is more, the decision-maker plays a central role in the application of science to practice, as he or she is responsible for the collection, critical 
appraisal, interpretation and fitting together of heterogeneous sources of evidence into a 'picture' of the specific situation. It is therefore unfruitful to strive for a practice that is 'objective' in the sense that it is detached from human involvement. To warrant the quality of decision-making in professional practice informed by science, it is more valuable to acknowledge the role of decision-makers and insist on their epistemological responsibility.

In this chapter, I have argued for epistemological responsibility as an alternative epistemology of clinical decision-making to EBM and have indicated different kinds of information and different types of reasoning that are involved in developing a 'picture' of a patient. In the following chapter, I will elaborate on my view of (medical) expertise that follows from this analysis. 
An epistemological shift: from evidence-based medicine to epistemological responsibility

\section{References}

Ankeny, R. (2010), 'Using Cases to Establish Novel Diagnoses: Creating Generic Facts by Making Particular Facts Travel Together', in How well do facts travel? The dissemination of reliable knowledge (ed.), How Well Do Facts Travel? (Cambridge: Cambridge University Press), 252-72 ISBN: 9780521159586 DOI: 10.1017/ cbo9780511762154.013.

Ankeny, R. (2014), 'The Overlooked Role of Cases in Causal Attribution in Medicine', Philosophy of Science, 81 (5), 999-1011 DOI: 10.1086/677693.

Bolt, T. and Huisman, F. (2015), 'Towards person-centered healthcare via realistic evidence-based medicine - informing the debate historically', 3, 275 DOI: 10.5750/ejpch.v3i3.1071.

Boon, M. and Knuuttila, T. (2009), 'Models as epistemic tools in engineering sciences: a pragmatic approach', in A. Meijers and Dov M. Gabbay (eds.), Philosophy of Technology an Engineering Sciences (Handbook of the philosophy of science; North Holland: Elsevier Science \& Technol), 687-720 ISBN: 978-0-444-51667-1

Cartwright, N. (1999), The Dappled Worlds: a study of the boundaries of science (Cambridge: Cambridge University Press) ISBN: 9780521644112.

Cartwright, N. (2011), 'A philosopher's view of the long road from RCTs to effectiveness', The Lancet, 377 (9775), 1400-01 DOI: 10.1016/S0140-6736(11)60563-1.

Cartwright, N. (2012), 'Will This Policy Work for You? Predicting Effectiveness Better: How Philosophy Helps', Philosophy of Science, 79 (5), 973-89 DOI: 10.1086/668041.

Charon, R. (2008), Narrative Medicine (Oxford: Oxford University Press) ISBN: 9780195340228.

Code, L. (1984), 'Toward a 'Responsibilist' Epistemology', Philosophy and Phenomenological Research, 45 (1), 29-50 DOI: $10.2307 / 2107325$.

Code, L. (1987), Epistemic Responsibility (99: Published for Brown University Press by University Press of New England) 123-26 ISBN: 087451407X

Djulbegovic, B. and Guyatt, G. H. (2017), 'Progress in evidence-based medicine: a quarter century on', The Lancet, 390 (10092), 415-23 DOI: 10.1016/S0140-6736(16)31592-6.

Dowding, D. and Thompson, C. (2009), 'Evidence-based decisions: the role of decision analysis.', in C. Thompson and D. Dowding (eds.), Essential Decision Making and Clinical Judgment for Nurses (London: Elsevier ), 173-95 ISBN: 9780702061622

Dreyfus, H. L. (2011), 'Medicine as Combining Natural and Human Science', The Journal of Medicine and Philosophy: A Forum for Bioethics and Philosophy of Medicine, 36 (4), 335-41 DOI: 10.1093/jmp/jhr027.

Greenhalgh, T. et al. (2014), 'Evidence based medicine: a movement in crisis?', BMJ, 348, g3725 DOI: 10.1136/ bmj.g3725.

Guyatt, G. H. et al. (1992), 'Evidence-based medicine: A new approach to teaching the practice of medicine', JAMA, 268 (17), 2420-25 DOI: 10.1001/jama.1992.03490170092032.

Guyatt, G. H. et al. (1995), 'Users' guides to the medical literature. IX. A method for grading health care recommendations', JAMA, 274 (22), 1800-04 DOI: 10.1001/jama.1995.03530220066035.

Hempel, C.G. (1965), Aspects of scientific explanation (New York: Free Press) ISBN: 0029143403

Howick, J. (2011), The Philosophy of Evidence-Based Medicine (West Sussex: Wiley-Blackwell) ISBN: 9781405196673.

Khushf, G. (1999), 'The Aesthetics of Clinical Judgment: Exploring the Link between Diagnostic Elegance and Effective Resource Utilization', Medicine, Health Care and Philosophy, 2 (2), 141-59 DOI: 10.1023/a:1009941101276.

Knuuttila, T. and Boon, M. (2011), 'How do models give us knowledge? The case of Carnot's ideal heat engine', European Journal for Philosophy of Science, 1 (3), 309-34 DOI: 10.1007/s13194-011-0029-3. 
Loughlin, M. (2008), 'Reason, reality and objectivity--shared dogmas and distortions in the way both 'scientistic' and 'postmodern' commentators frame the EBM debate', J Eval Clin Pract, 14 (5), 665-71 DOI: $10.1111 / j .1365-2753.2008 .01075 . x$.

Loughlin, M. (2009), 'The basis of medical knowledge: judgement, objectivity and the history of ideas', J Eval Clin Pract, 15 (6), 935-40 DOI: 10.1111/j.1365-2753.2009.01318.x.

Miles, A. (2018), 'Evidence-based medicine - 2018. Quo Vadis?', Journal of Evaluation in Clinical Practice, 24 (1), 3-6 DOI: 10.1111/jep.12924.

Montgomery, K (2006), How Doctors Think: Clinical Judgment and the practice of medicine (New York: Oxford University Press) ISBN: 9780195187120.

Parkinnen, V-P. et al. (2018), Evaluating Evidence of Mechanisms in Medicine. Principles and Procedures (SpringerBriefs in Philosophy; Dordrecht: Springer) ISBN: 978-3-319-94609-2.

Sackett, D. L. et al. (1996), 'Evidence based medicine: what it is and what it isn't', BMJ, 312 (7023), 71-72 DOI: 10.1136/bmj.312.7023.71.

Schön, D. (1983), The Reflective Practitioner - How professionals think in action. (New York: Taylor \& Francis Ltd.) ISBN: 978-0465068784.

Solomon, M. (2008), 'Epistemological reflections on the art of medicine and narrative medicine', Perspect Biol Med, 51 (3), 406-17 DOI: 10.1353/pbm.0.0038.

Tonelli, M. R. (1998), 'The Philosophical Limits of Evidence-Based Medicine', Academic Medicine, 73 (12), 7

Toulmin, S. (2001), Return to Reason (Cambridge, MA \& London: Harvard University Press) ISBN: 9780674012356.

Vist, G.E. et al. (2004), 'Grading quality of evidence and strength of recommendations', BMJ, 328 (7454), 1490 DOI: $10.1136 / \mathrm{bmj} .328 .7454 .1490$.

Worrall, J. (2002), 'What Evidence in Evidence-Based Medicine? ', Philosophy of Science, 69 (S3), S316-S30 DOI: $10.1086 / 341855$.

Wyer, P. C. and Silva, S. A. (2009), 'Where is the wisdom? I--a conceptual history of evidence-based medicine', J Eval Clin Pract, 15 (6), 891-8 DOI: 10.1111/j.1365-2753.2009.01323.x. 


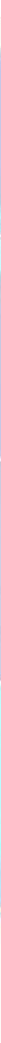




\section{Evidence-Based Medicine versus Expertise: knowledge, skills and epistemic activities}

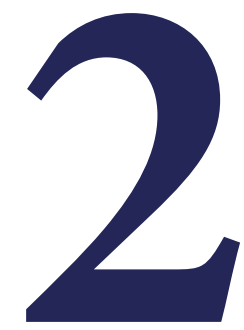

This chapter is adapted from: Van Baalen, S. \& Boon, M. (2017) Evidence-Based Medicine versus Expertise: knowledge, skills and epistemic activities. In: Bluhm, R. (ed.) Knowing and Acting in Medicine. Rowman \& Littlefield. ISBN: 978-178348810

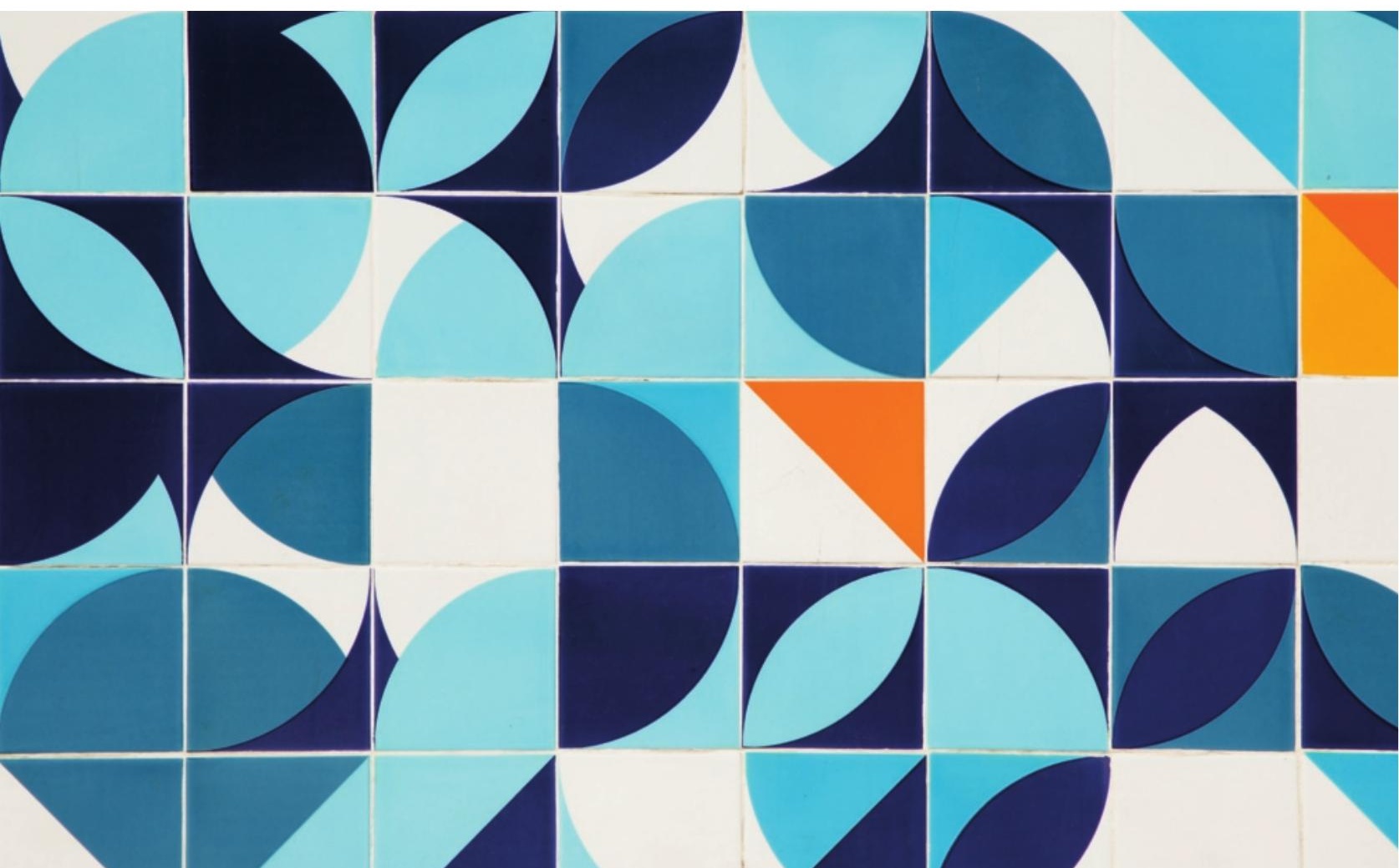




\section{Abstract}

Acknowledging the role of the knower in the construction of scientific knowledge and the use of science in practice, as is argued for by critics of EBM as well as by several contemporary philosophers of science, requires turning attention to (medical) expertise. A more detailed account of expertise will give guidance to the epistemological responsibility of doctors, in terms of how it can be developed and how it can be assessed. I follow Collins and Evans (2007) in their assertion that expertise is about what a person can do rather than what they know and that 'tacit knowledge' plays a key role in expertise. However, I expand on their ideas by arguing that an account of medical expertise should also include a doctor's competence in performing epistemic activities. Following Hasok Chang (2012, 2014), I interpret clinical decision-making as a 'system of practice' that consists of three general epistemic activities: gathering and assessment of information; constructing a 'picture' of a patient that can be used as an epistemic tool for reasoning about diagnosis; and, the adaption and application of this epistemic tool. I argue that being competent in performing epistemic activities requires cognitive skills, and similar to physical skills, mastering cognitive skills entails internalization or tacit knowledge. However, I do not think of tacit knowledge as inarticulate and therefore hidden for evaluation. Instead, I revisit Michael Polanyi's $(1958,1966)$ original, richer and more detailed conception of tacit knowledge. Polanyi proposed a distinction between subsidiary and focal attention and explained that knowledge in one's subsidiary awareness can be opened up to scrutiny. I adopt this idea in my account of expertise. It means that the use of knowledge in systematic reasoning by experts is largely tacit, but that it can be brought to their focal attention thus allowing for its explicit justification. Accordingly, doctors can and should be held accountable for how well they perform epistemic activities in decision-making. This entails that they can be held responsible for acquiring, developing and maintaining competence in cognitive skills and that they bear responsibility for the quality of the knowledge and reasoning. This is what I have called their epistemological responsibility. 


\subsection{Introduction}

Since its inception in the early 1990's, evidence-based medicine (EBM) has been promoted as a way to make clinical practice more scientific, though its epistemology has been criticized on many grounds, for example for being based on a narrow view of science that focuses on quantitative, clinical evidence and rule-following instead of basic science, theories and judgments (Loughlin 2008, 2009; Tonelli 1998; Worrall 2002; Wyer and Silva 2009). In Chapter 1, I have provided an overview of these critiques and some of the reactions from EBM proponents. In that chapter, my main issue was EBM's preference for only one type of scientific knowledge (produced in comparative clinical studies) over other sources of information and EBM's envisioned algorithmic style of clinical reasoning prescribed by guidelines. This chapter will focus on another line of critique, that is, EBM's disregard of 'expert opinion,' in particular the role of physician's expertise in clinical decision-making.

Very shortly after EBM was announced as a "new paradigm for medicine" (Guyatt et al. 1992), Sandra Tanenbaum (1993) pointed out some of its philosophical challenges, among which is a misrepresentation of clinical reasoning: "In an act of interpretation, not application, physicians make clinical sense of a case, rather than placing it in a general category of cases. As interpreters, physicians draw on all their knowledge, including their own experience of patients and laboratory-science models of cause and effect," (Tanenbaum p. 1269). She argues that clinical medicine inherently has to deal with the uncertainty of a situation and with incomplete information. For physicians to make wise decisions requires them necessarily to rely on 'personal knowledge,' including experience and sensory input. In a similar fashion, Mark Tonelli (1998) defended 'expert opinion' by first arguing that expertise is wrongfully put on the lowest rung in the 'hierarchy of evidence' ladder because it is different in kind rather than in degree from the other types of evidence that EBM ranks. According to him, expert opinion is crucial to overcome the epistemic gap between the outcomes of population-based clinical trials and those of the individual patient, as well as the normative gap between what is regarded as the 'best' treatment according to EBM (i.e., the treatment that gives the best outcome to a group of patients with a particular disease in a clinical trial) and what will be the 'best' treatment for an individual patient. With a clinical case description, Tonelli (2006) illustrates how an experienced physician uses "all relevant kinds of medical knowledge, along with patient goals, values and preferences, in order to reach the best possible decision for the patient-at-hand," (Tonelli 2006, p. 73). The role of expertise in such decision-making requires a physician to be aware of the strengths and weaknesses of each kind of medical knowledge and to cope with the 
fact that evidence derived from clinical research cannot be prescriptive but still requires experience and pathophysiological knowledge.

One of EBM's initial aims was to improve the quality of clinical decision-making by minimizing bias, subjectivity and uncertainty through statistical evidence and rule-based reasoning (Guyatt et al. 1992). As Tanenbaum (1993) and Tonelli $(1998,2006)$ argue, EBM falls short in its initial aims when neglecting the crucial role of expert opinion in making clinical decisions about individual patients. Together with other critiques, this has led proponents of EBM to ask whether it is "a movement in crisis" and to contend that "evidence-based medicine has not resolved the problems it set out to address (especially evidence biases and the hidden hand of vested interests)" (Greenhalgh, Howick and Maskrey, p. 5). Greenhalgh, Howick and Maskrey (2014) argue that in order for EBM to overcome this crisis it needs to refocus its attention to training doctors to use intuitive reasoning based on experience, followed by formal EBM methods to "check, explain, and communicate diagnoses and decisions," and to share uncertainty with patients. Furthermore, they argue that drawing on a "wider range of underpinning disciplines," including qualitative research, would enrich the field. In other words, EBM proponents admit that EBM falls short as a guiding principle for clinical decision-making. Tonelli (1998), in turn, admits that the danger of overly relying on the authority of medical experts is that experts may become 'authoritarian,' rigid and not receptive to new evidence and insights. In addition, expert opinion does not necessarily provide good grounds for decision-making, as experts can disagree or have biased opinions. Thus, opponents and proponents of EBM are showing signs of rapprochement in the sense that both sides acknowledge that evidence from clinical research alone is not sufficient to make diagnosis and treatment decisions for individual patients, whereas reliance on expert opinion holds the danger of an unwanted return to 'authority-based' decision-making. The challenge that is raised by this rapprochement is to develop an epistemology of clinical decision-making that acknowledges the central role of medical expertise in decision-making, but at the same time allows us to assess the quality of knowledge and reasoning in this these practices.

In the philosophy of science, several authors have argued for a greater involvement of the 'knower' in the study of science, in contrast to traditional positivist approaches to philosophy of science. In Chapter 1, I have elaborated on Lorraine Code's argument that cognitive agents are active in forming their beliefs, and that a knower has an important degree of choice in cognitive processing, for which they can (and should) be held accountable (Code 1984). More recently, similar ideas have been taken up by other authors as well. For example, Hasok Chang (2012) argues that ("standard Anglophone") philosophy of science has been limited by "viewing science as a body of propositions, focusing on the truth-value 
of those propositions and the logical relationships between them" (Chang 2012, p. 67). Therefore, he proposes to take the concepts of 'epistemic activity' and 'system of practice' as units of analysis (more about these concepts later). According to Chang (2014), this also requires a "full and coherent account of the epistemic agent" (Chang 2014, p.70). This involves recognizing that agents have a purpose from which they act, that are shaped by the agent's beliefs and desires, as well as a degree of freedom, for example in choice of theory, and capabilities and limitations. ${ }^{9}$ Henk de Regt (2015) argues that the idea that scientific theories provide understanding by virtue of them being true descriptions of the world conflicts with the reality of scientific practice. De Regt illustrates this by providing examples in life sciences and social sciences in which theories or models can be unrealistic but nevertheless productive for understanding, as they allow making inferences about a target system and to predict and control its behaviour. As an alternative, De Regt characterizes understanding as contextual and depending on skills. In his alternative conception, scientific theories or models are not objectively true, but rather true with regard to a particular context and purpose. Using theories or models for understanding does not depend on them being accurate representations of phenomena, but rather their intelligibility, which is in turn dependent on the skills of the person working with them. ${ }^{10}$ Hence, for De Regt the knower and his or her ability to perform epistemic activities, plays a crucial role in scientific understanding. Finally, Mieke Boon (Boon 2017a) argues for 'epistemological constructivism' in which the central question is "how is the construction of knowledge for epistemic uses possible?” (Boon 2017a, p.293). According to Boon, mainstream philosophy of science regards the construction of knowledge as belonging to the context of discovery and therefore not as something that philosophy of science is concerned with (which is the 'logic of knowledge', or the context of justification). Additionally, philosophers of science (such as Hempel 1965) implicitly assume that concrete problems can be solved by deductive reasoning from scientific laws and theories. In contrast, Boon argues that construction, justification and application of knowledge are entangled: “Constructing [...]

9 "Rational decisions should be based on an accurate sense of the agent's own capacities and skills," (Chang 2014, p. 71)

10 De Regt elaborates on 'intelligibility' and skills in (De Regt 2009). Here, he regards skills as “the ability to construct deductive arguments from the available knowledge. [...] the ability to make judgments about how to proceed without an algorithm" (De Regt 2009, p. 26, his emphasis). In addition, he argues that, usually, scientific explanations involve more than simple deduction. Rather, theories first have to be contextualized through a process of modeling that involves approximation and idealization and for which there are no formal algorithms or formal principles. Relevant skills thus also include those that are needed to build models, which depends on the context and the theories a scientist wants to use. 
involves its justification, not only in view of established knowledge and data, but also in view of intended applications. Using it (in new, real-world situations) involves assessment and justification of whether it suits this particular application, but also constructive activities [...] the two aspects of using knowledge require an understanding of its original construction, such as the idealizations and simplifications" (Boon 2017a p. 296). Boon refers to Kant's synthetic a priori as the 'conditions for the possibility' of knowledge or regulative principles ${ }^{11}$ to argue that knowledge production is the product of a tri-partite relationship between world, knowledge and human cognition and that the role of the user and producer of knowledge cannot be eliminated in philosophy of science, as their cognitive make up, philosophical presuppositions (as expressed in regulative principles), and intended epistemic goals play an ineliminable role in how experiences are conceptualized and structured.

In short, within the philosophy of science there is a movement that touches upon a similar discussion and similar dilemmas as EBM epistemology, revolving around the question if and in what way the knower plays a role in the construction and application of knowledge and, if so, what epistemic criteria should be used to evaluate this. In view of this dilemma, the aim of this chapter is to explicate what aspects of medical expertise allow doctors to make sound clinical decisions, and reflect on this as a case of application of science in practice. I will argue that medical expertise does not consist only of formal knowledge, practical skills or experience (or any combination of these traits), but should also include cognitive skills. In other words, medical expertise also involves the ability to perform the epistemic activities needed to produce adequate knowledge about individual patients, by combining knowledge and experience from different sources fitted to the specific situation of that patient. Therefore, becoming an expert involves learning to use scientific knowledge and medical evidence in clinical-decision making. This is not, however, simply something that is tacitly learned by being immersed in the tradition and authority of existing medical practice. Instead, expertise means that clinicians can actually justify their decisions which requires, as I will argue, that experts can justify how they reach their decisions.

Yet, this idea is seemingly at odds with authors who, when defending the importance of expertise in clinical decision making, emphasize the role of tacit knowledge in these reasoning processes (Henry 2010; Malterud 2001). My point will be that tacit knowing

11 Regulative principles that Boon mentions are: the principle of induction; the principle that every event has a cause; the principle that nature forms a system according to laws; and the principle of unity of nature. (Boon 2017, 299) 
and reasoning in these accounts are regarded as inarticulate and therefore inaccessible. As a consequence, clinical decision-making remains a mysterious and vague process that cannot be reflected on, running into the danger that the original problem of tradition and authority-based decision-making will persist. In order to address this problem, I will analyse the notion of tacit knowledge and its role in expertise by starting from Collins' and Evans' (2007) skill-based account of expertise. Next, in order to open up and evaluate the tacit dimension in their notion of expertise I will revisit Polanyi's (1958) original account of tacit knowing. As I will show, Polanyi's more subtle notion allows for better understanding how clinicians can indeed be held accountable for their decisions. I have labelled this type of accountability the epistemological responsibility of clinicians (see Chapter 1) which I consider an inherent aspect of an enriched understanding of medical expertise.

Based on my analysis of the role of tacit knowledge in expertise, in Paragraph 2.6, I will explain in more depth the meaning of epistemological responsibility of clinicians as a way to overcome the dangers of tradition and authority-based decision-making. The proposed account of expertise in terms of epistemological responsibility is relevant for two reasons, first in understanding how the quality of clinical decision-making can be accounted for, and second in specifying learning-aims of the education of medical students. Finally, the framework of medical expertise that I present here can also provide a more general guide to evaluating epistemic skills in other scientific research practices and in practices in which science-based judgments are made. An epistemology that emphasizes the role of the expert and the responsibility that this entails can help to deal with the fear of subjectivism and relativism in those practices.

\subsection{Epistemological responsibility in clinical practice}

In Chapter 1 I have argued that medical doctors have a professional responsibility to come up with the best possible diagnosis and treatment plan for individual patients, by generating knowledge about the disease of a particular patient. To meet this responsibility, the key epistemological challenge of doctors involves gathering and integrating all relevant yet heterogeneous sources of information, including not only scientific-medical knowledge of diseases and treatments, and diagnostic data on the patient, but also contextual information (e.g., the patient's situation, goals and values, the availability of specific medical equipment and expertise and the constraints of the medical system) so as to construct a coherent 'picture' of the patient's disease and its possible treatments. 
In this chapter, I add that this 'picture' must be constructed such that it can be used as an epistemic tool, which means to say that it must be constructed such that it enables further reasoning about the patient's condition, for instance, in formulating alternative hypotheses on the disease (the diagnosis), predicting which cure may work (the treatment-plan), or in explaining why a treatment causes side-effects.

The notion of 'epistemic tools' for reasoning about a target-system was coined by Mieke Boon and Tarja Knuuttila (Boon and Knuuttila 2009; Knuuttila and Boon 2011) who propose the notion of scientific models as epistemic tools as an alternative to a generally held representational notion of scientific models. Boon (Boon 2017b) aligns the notion of 'knowledge as epistemic tool' with an engineering paradigm of science. The core of this paradigm is, according to Boon, the epistemic aim of producing useful knowledge for solving problems external to the scientific practice, in contrast to the physics paradigm of science, which presupposes a representational notion of knowledge. In the representational notion of knowledge, the aim is to produce true knowledge (rather than useful or applicable knowledge) that corresponds with the object of study, believing that 'true' knowledge would be automatically useful. Therefore, the physics paradigm of science is also associated with reductionist metaphysical and epistemological presuppositions. Boon shows that reductionist approaches are limited in the biomedical sciences because they do not accommodate an understanding of how studied entities interact with the environment or function in a large, complex system. Therefore, she proposes to characterize knowledge produced for problem-solving and creating new things and functions (as in the engineering paradigm of science) as epistemic tools. In this view, knowledge is constructed (by epistemic agents) with specific epistemic aims in mind.

The analogy I wish to draw is between scientific models and the 'picture' of a patient. According to Knuuttila and Boon (2011), the construction of a scientific model involves constructing a coherent whole out of relevant but heterogeneous epistemic sources for a specific epistemic purpose. Knuuttila and Boon claim that the process of constructing scientific models (the modelling) is already part of the justification of the model, because constructing a model - or in my case the 'picture' of the patient - involves justification of the information that is built into this 'picture' (e.g., information on the individual patient and more general theoretical and clinical information need to be relevant as well as reliable) and requires that these heterogeneous bits of information are coherently tied together. In the vocabulary of this chapter one could say that modellers have the expertiseknowledge, experience and cognitive skills - to build models that are at least partly correct about the specific target-system for which the model is constructed. When translated to the medical context, the scientific model (the 'picture') constructed by the modeller (a 
medical doctor) is about a specific target-system (an individual patient), and functions as an epistemic tool that allows for scientific reasoning about the target-system. Hence, instead of truly representing the target-system, the scientific model is a tool that allows for scientific reasoning about the system. Similarly, instead of finding objective 'truth' about the individual patient by scientific approaches such as EBM, 'pictures' of individual patients constructed by medical experts allow for reasoning about the patient in clinical decision-making, for instance, in formulating relevant and testable questions and hypotheses that eventually guide towards proper diagnoses, in predicting which cure may work as part of the treatment plan, or in explaining why a treatment causes side effects. In clinical practice, the concept 'model' has a different connotation, therefore the word 'picture' is used to characterize similar epistemic processes.

When general knowledge and specific information are brought together to form a coherent picture of an individual patient in a specific context, doctors develop an epistemic tool that enables them to reason about this patient in the best possible way. The objectivity and scientific quality of this epistemic tool is not related to truth but to its relation to other relevant epistemic criteria, such as logical consistency and coherency with relevant knowledge (including knowledge generated in RCTs). Another important epistemic criterion is its utility for this specific situation: to make the best possible diagnosis and treatment decisions for the individual patient, and for the specific situation. For example, if a doctor has more or better experience with one treatment for a certain case, whereas the results of RCTs suggest another, the treatment favoured in the RCTs may not be the most appropriate. In that case, doctors can argue to opt for a different choice of treatment, for example by referring to their personal success rates with that specific treatment or specificities of the case that make that treatment more appropriate than another. Therefore, contextual and personal information, like the availability of a certain device in a hospital, a doctor's experience, and a patient's preferences are all relevant because they have an impact on what is the best decision in a specific situation.

Due to the heterogeneous character of the various aspects playing a role in the construction of an epistemic tool, it is not possible to construct this tool by means of rule-based reasoning only. Instead, constructing an epistemic tool (the 'picture' of a patient) requires complex reasoning and assessment of evidence. Therefore, in Chapter 1 I have proposed that, instead of deferring responsibility to general clinical guidelines, as at least some interpretations of EBM seem to suggest, doctors should consider themselves epistemologically responsible for producing good quality diagnosis and treatment. I argue that the epistemological tasks of doctors involve a considerable amount of choice, deliberation and justification, for which they are held responsible in our account of medical decision-making. 
In other words, by introducing the concept of epistemological responsibility, I allow for a richer and less rigid epistemology of clinical decision-making that leaves space for alternative modes of reasoning better suited to the epistemological challenges of clinical decision-making. However, in spite of the space for personal judgments by medical experts defended here, I wish to avoid a return to the justification of decisions on the basis of cognitively empty authority or 'professional opinion.' By introducing the notion of epistemological responsibility, I point at specific responsibilities of clinicians towards the best possible execution of epistemic activities in diagnosis and treatment, and at the accountability of clinicians for the quality of their decisions. Specific skills are required to perform epistemic activities responsibly, and these skills are an important aspect of medical expertise. A further characterization of these skills will therefore help clarify what it means for a clinician to be epistemologically responsible and to help assess the quality of expert decision-making.

In this chapter, I introduce the notion of 'a 'picture' of a specific patient as an epistemic tool for reasoning' in regard of the diagnosis and treatment of that patient (see Chapter 1). Similar to the paradigm shift between the physics paradigm and the engineering paradigm described by Boon (2017b), I will shift the focus from 'epistemic truth' and passive rule-following to 'epistemic use' and active knowledge-construction. Within the epistemological responsibility framework of medical decision-making, it makes sense to focus on what doctors actually do when they generate knowledge of a patient and make diagnostic and treatment decisions, in other words to the epistemic activities they perform.

Parallel to my concern with the epistemic activities performed by clinicians in medical practice is Hasok Chang's $(2012,2014)$ proposal that philosophy of science should concern itself with what scientist $d o$, hence focus on activities instead of only on theory as a set of propositions. In the paragraph 'epistemic activities' later in the chapter, I will give a more detailed account of Chang's notion of empirical activities and add my understanding of empirical activities in clinical decision-making.

In short, in Chapter 1, I have argued that clinicians are epistemologically responsible for constructing a 'picture' of each individual patient from heterogeneous sources of information. Additionally, in the previous paragraphs of this chapter, I have argued that constructing a 'picture' of the patient as an epistemic tool for clinical reasoning is a demanding epistemic task. For doctors, students and policy-makers to know what a clinician's accountability involves when expecting doctors to bear responsibility for this epistemic task requires a detailed account of the kind of expertise needed in these tasks - in particular, how this competence is developed, how epistemic activities relate to medical 
expertise, and how the performance of this task can be assessed. My framework provides useful leads for developing such an account: by shifting the attention from what is known to the knower I return to a more doctor-centred account of clinical decision-making, and by focusing on epistemic tools and epistemic activities, I point out that what matters for clinical decision-making is not only the knowledge and information that is used but also what doctors do with it, in other words, how that knowledge and information are used in the reasoning process to reach clinical decisions. Performing activities and using tools well requires skills that are developed as a part of a professional's expertise. In explicating how clinical expertise is developed and what it means to develop skills in performing epistemic activities, I will first evaluate Collins and Evans' (2007) skill-based account of expertise.

\subsection{Medical expertise}

Collins and Evans have developed a view of expertise in which expertise is not solely defined by the amount of formal knowledge possessed by individuals, but as something practical: ${ }^{12}$ "something based in what you can do rather than what you can calculate or learn" (Collins and Evans 2007, p. 23). Central to their thesis on expertise is an immersion within a certain community of experts, which is necessary in order to gain expertise. They distinguish between two levels of specialist expertise, ${ }^{13}$ interactional and contributory expertise. They define interactional expertise as "expertise in the language of a specialism, in the absence of expertise in its practice" (ibid, p. 28). In other words, only immersion in

12 Collins and Evans introduce this 'wisdom-based' or 'competence-based model' as an alternative to the logical and mathematical way that expertise has been understood by philosophers and sociologists up to about a half-century ago by philosophers and sociologists. Collins and Evans' account finds its origin in ideas from phenomenological philosophers such as Heidegger and Merleau-Ponty, and ideas from philosophers like Polanyi (and his concept 'tacit knowledge' - see later in this chapter) and Wittgenstein (and his idea that the meaning of a concept can be understood only through its use). The other approach to expertise focuses on the cognitive state of experts, defining expertise in terms of the amount of true and false beliefs within a domain that a person possesses (Goldman 2016). This can be relative to others ("an expert has more true and less false beliefs about a domain than other people") or in absolute terms ("an expert has a substantial amount of true beliefs about a domain") or both at the same time.

13 Collins and Evans contrast "specialist expertise" to "ubiquitous expertise", the latter including "all the endlessly indescribable skills it takes to live in a human society." (Collins and Evans, 2007, p.16). They argue that ubiquitous expertise requires ubiquitous tacit knowledge, which is acquired through immersion in society in general, whereas specialist tacit knowledge requires immersion in a community of specialists. 
the world of language is needed to acquire this kind of expertise, which requires only a 'minimal body' that only fulfils the requirements that are necessary to learn a language. This is what they call "the minimal embodiment thesis" (ibid, p. 79). In contrast, they define contributory expertise as "enabling those who have acquired it [contributory expertise] to contribute to the domain to which the expertise pertains: contributory experts have the ability to do things within the domain of expertise" (ibid, p. 24). According to Collins and Evans, this requires full embodiment ${ }^{14}$ and immersion in a community of experts in a domain.

Collins and Evans point out that all specialist expertise (interactional as well as contributory) involves a great deal of specialist tacit knowledge, defined by them as "things you know how to do without being able to explain the rules for how you do them" (ibid, p. 13). Therefore, in order to acquire any degree of specialist expertise requires interactive immersion in a specialist culture or enculturation "because it is only through common practice with others that the rules that cannot be written down can come to be understood" (ibid, p. 24). For medical specialists, this would mean that to master medical expertise requires to be immersed in the culture of day-to-day medical practice. An endorsement of this idea is the extensive system of apprenticeship teaching in the education of medical professionals: a large part of what a clinician learns is learned by "doing" in internships, residencies and fellowships. Collins and Evans present a five-stage model of the acquisition of contributory expertise - adopted from Dreyfus and Dreyfus (1986) - in which successive steps (from novice to expert) represent an increasing internalization of physical skills, exemplified by the process of learning to drive a car (ibid, p. 24-25). This process hinges on the acquisition and mastering of skills, unselfconsciously recognizing contexts and unselfconsciously acting accordingly. This unselfconscious decision-making in response to a certain context is what is considered to be 'tacit knowledge' by Collins and Evans. In addition, Collins and Evans emphasize that to understand expertise one should focus on what experts do instead of what they know.

14 One thing that might be argued for when discussing medical expertise is that only a minimal body is required for epistemic activities, and therefore, people with only interactional medical expertise are capable of performing clinical judgment. However, the point that I want to make in this paper is not that medical expertise is a particular kind of expertise that can be fully characterized by considering the relevant epistemic activities. Rather, in order to fully explicate medical expertise, our account of expertise should be widened to include, besides knowledge and physical skills, also cognitive skills. 
In short, in Collins' and Evans' account of what makes somebody an expert in a certain specialism involves - besides being familiar with the epistemic content - skills and enculturation, which in the case of high-level experts are largely tacit. Their account presents important reasons for the crucial role of (medical) expertise, but may unintendedly vindicate non-transparent authority-based reasoning in medical decision-making. Therefore, in my view, it has two shortcomings. First, it does not address the acquisition and cultivation of cognitive skills required to perform epistemic activities, and second, it does not provide an adequate account of tacit knowledge to analyse the quality of medical reasoning.

\subsection{Epistemic activities}

In everyday clinical practice, doctors are continuously performing epistemic activities, for example hypothesizing which treatments will be beneficial, predicting the risk of adverse effects, or adjusting treatment plans and interpreting new information in light of what is already known about a patient. Collins and Evans, in their examples and explanation of their account, focus mainly on the acquisition of physical skills and on enculturation (i.e., understanding and use of language and unwritten social rules) into the relevant group practices. Although I agree with them that in medical practice skills (such as surgical skills and communication skills), epistemic content (such as basic biomedical knowledge, knowledge of treatments and up-to-date knowledge of clinical science) and acquaintance with the medical (hospital) culture (e.g., traditions, hierarchy and behavioural etiquette) are all highly important, my point is that focusing on these aspect of expertise leaves out another aspect that is particularly crucial for clinical decision-making, which is the ability to perform specific epistemic tasks and activities related to medical reasoning at an expert level.

Hasok Chang (2012) develops a 'framework for activity-based analysis' in which he defines an epistemic activity as a "more-or-less coherent set of mental or physical operations that are intended to contribute to the production or improvement of knowledge in a particular way, in accordance with some discernible rules (though they may be unarticulated)," (Chang 2012, p. 16). Crucial for this framework is the presence of an identifiable aim as succeeding in this aim is crucial to assess the coherence of an activity. In addition, (a coherent set of) epistemic activities occur in a 'system of practice' and, similar to the coherence of epistemic activities, "it is the overall aims of a system of practice [which go beyond the specific purposes of the activities that make up the system] that define what it means for the system to be coherent" (ibid, p. 16). Coherency, for Chang, "goes beyond mere consistency between the propositions involved in its activities; rather, coherence 
consists in various activities coming together in an effective way toward the achievement of the aims of the system" (ibid, p. 16). According to Chang, focus on activities and aims also allows for normative evaluations of scientific practices by making overall evaluative notions (such as truth and success) applicable to the concrete context of an epistemic activity. Hence, focusing on epistemic activities opens up the possibility to evaluate the quality of decision-making without having to defer to overly general rules or concepts. Rather, focusing on specific epistemic activities also allows defining specific criteria for specific contexts and requires an analysis of what abilities one should have in order to perform epistemic activities concerning good quality clinical decision-making. Going back to Collins's and Evans's skills-based notion of expertise, it becomes evident that, just as expertise in a certain specialism involves a specific set of physical activities that a person should master, it also involves a set of epistemic activities that experts are able to perform skillfully. Consequently, Collins and Evans's ideas about the acquisition of expertise through experience and apprenticeships should be extended to include the acquisition and deployment of cognitive skills.

Epistemic activities that Chang mentions are: describing, predicting, explaining, hypothesizing, testing, observing, detecting, measuring, classifying, representing, modelling, simulating, synthesizing, analysing, abstracting, idealizing (Chang 2012), counting, narrating and ordering (Chang 2009). This illustrates that epistemic activities can, in turn, be composed of other epistemic activities (i.e., testing might be composed of observing and measuring). Chang himself admits that "the activity-system distinction is indeed relative and context-dependent; a system of practice, if it has a clear inherent purpose, can be taken as a single activity that may form part of a larger system" (Chang 2012, p. 75). In addition, Chang stresses the non-reductive nature of his framework: component activities are not necessarily simpler than the main activity (Chang 2014). Adopting Chang's framework, I interpret clinical decision-making in general as a 'system of practice' in which several levels of epistemic activities can be identified, described and assessed. I identify three main activities consisting of several component activities: gathering and assessment of information; constructing the epistemic tool in the sense of a 'picture' of the patient; and the adaptation and application of 'picture' of the patient.

Each of these activities consists of yet other epistemic activities. The epistemic activities 'gathering and assessment of information' involve activities such as asking the patient questions about his or her signs and symptoms, performing measurements and identifying scientific knowledge. To perform such activities also requires the clinician performing activities that amount to identifying information that is both reliable and relevant to the specific situation. For example, formulating questions; reasoning about possibilities and 
limitations of measurement technologies; and, critical appraisal of scientific knowledge. 'Constructing the epistemic tool' in the sense of a 'picture' of the patient involves activities such as the interpretation of measurements; explaining an observation, for instance, by 'subsuming' an observed phenomenon under a general law; contextualizing; ${ }^{15}$ and drawing creative relationships between different types of information. Finally, 'further reasoning using the epistemic tool,' involves asking new kinds of questions; crafting hypotheses; conceptualizing aspects of the 'picture' of the patient; constructing models of aspects of the target-system (partially based on those conceptualizations); and, coming up with new kinds of possible or necessary interventions and their expected outcome.

Performing these activities is not a matter of simple execution but require a lot of coordination and fine-tuning, keeping in mind the overall and specific aims of the activities and system of practice. In other words, specific abilities ${ }^{16}$ are required to perform these activities, which are cognitive in nature. Being able to perform these activities thus require a specific type of skills that are different from physical skills or communicative skills, i.e., cognitive skills. Expert clinical decision-makers thus need to master specific cognitive skills, and following Collins and Evans, mastering cognitive skills at an expert level involves having tacit knowledge. In Paragraph 2.6 I will show this for the epistemic activities involved in clinical decision-making. However, as the activities and abilities outline mentioned above for a large part take place in the mind, referring to tacit knowledge as "things you know how to do without being able to explain the rules for how you do them" does not bring evaluation of the quality with which such activities are performed any closer. I will address this problem in the next section.

\subsection{Tacit knowledge}

The second shortcoming of Collins and Evans's account of expertise is their notion of tacit knowledge. They assign a pivotal role to tacit knowledge in expertise, for example by describing expertise as an ability to tacitly make decisions in response to a certain situa-

15 This could mean two different things: (a) attributing details relevant to the specific context, (b) subsuming the specific problem under relevant scientific disciplines to perform further epistemic activities.

16 These abilities are, for example, to articulate the patient's problem (i.e., complaints, signs and symptoms); to consider which information is relevant for further thinking about this problem; to identify what information is missing that is required to make reliable judgments; to foresee the possible (positive and negative) consequences of an intervention; and, to find explanations for unexpected effects. 
tion or context. However, in their definition, tacit knowledge is inarticulate and therefore inaccessible for inquiry and evaluation. As a consequence, their account obscures an analysis of what it involves to make these decisions and what it means to be an expert in that respect. The problem with thinking about the skilful execution of epistemic actions in this way seems to be that expert decision-making and the knowledge used in the process become increasingly inaccessible to others, which increases the likelihood of referring to authority rather than epistemic quality in clinical decision-making.

In contrast, a fruitful interpretation of 'tacit knowledge' in clinical reasoning for diagnosis and treatment would focus on what kind of knowledge is used and how reasoning is performed tacitly so that its strength, relevance and application can be evaluated. For example, Michael Loughlin (2010) argues that "features of our knowledge that function tacitly in many contexts can, without contradiction, be made the object of explicit attention in others" (Loughlin, 2010, pg. 298). Loughlin argues that it is a mistake to assume that tacit knowledge is completely inarticulate in all situations. Stephen Henry (2010), analysing how a physician in a neurological exam focuses on observing neurological symptoms while being tacitly aware of a patient's body part, writes: "Whether information is tacit or explicit has less to do with its content than it does with how it functions in a particular situation" (Henry 2010, pg. 294). In other words, tacit knowledge is not essentially different from explicit knowledge, and therefore, knowledge that functions tacitly in one situation can be reconstructed in another situation to study how it informs clinical decision-making. Hence, an account of tacit knowledge in medical reasoning need not be implicit and mysterious.

Summing up, Collins and Evans show that tacit knowledge plays a pivotal role in expertise, but their view of tacit knowledge as inarticulate does not clarify how to evaluate the reasoning process. Referring to inarticulate tacit knowledge as a justification for clinical decisions and actions is not enough to hold clinicians accountable for the decisions they make. In order to ensure the quality of clinical decision-making for diagnosis and treatment, a better understanding of tacit knowledge is needed, which is why I will revisit the original definition by Michael Polanyi.

\subsubsection{Polanyi's tacit knowledge}

Michael Polanyi first introduced the concept 'tacit knowledge' in his book Personal Knowledge: Towards a Post-Critical Philosophy (1958) and further explicates this notion in The Tacit Dimension (1966). His idea has been widely taken up to make sense of situations in which experience, know-how, and practice play important roles. For example, the notion of tacit knowledge has been important in the (sociological) analysis of scientific practices, 
such as gravitational physics labs, and the realization that in such practices, - in addition to knowledge, theories and concepts - skills and personal contacts are crucial (for example see: Collins 2001). However, over the years, tacit knowledge has come to mean 'inarticulate, and therefore as not transferable to others and inaccessible by others. This conception of tacit knowledge leads to obfuscating and mystifying expertise and is therefore seemingly unsuitable as a concept to clarify expert knowing. In contrast, Polanyi's original notion is much more detailed and refined and therefore appears to be very helpful in better understanding expertise.

In Polanyi's epistemology, knowing comprises two types of awareness: the subsidiary and the focal (Polanyi 1958, p. 57, also see: Mitchell 2006). Focal awareness is the conscious object of our awareness, whereas subsidiary awareness includes a variety of background clues that enable focal awareness. An example to understand these two kinds of awareness is the recognition of somebody's face: “we know a person's face, and can recognize it among a thousand [...] Yet we usually cannot tell how we recognize a face we know" (Polanyi 1966, p. 4). Polanyi goes on to explain that faces consist of a collection of particular physiognomics such as a nose, a mouth, eyes, ears, etc. In recognizing a face, we are not consciously aware of all the particulars of a face, but rather by integrating our awareness of its particulars, as subsidiaries, in order to recognize the whole, the face that is our focal awareness. This is, according to Polanyi, "the outcome of an active shaping of experience performed in the pursuit of knowledge" (ibid, p. 6). Therefore, in tacit knowing, the knower is the third essential element. These three elements make up a from-to relationship: the knower "attend[s] from the subsidiaries to the focal target" (Mitchell 2006, p.73).

According to Polanyi, the use of tools (e.g., technological instruments such as a probe us for ultrasound imaging) also involves 'incorporation' in our subsidiary awareness. He calls this process, in which the use of a tool moves from focal to subsidiary awareness, 'indwelling.' For example, when an ultrasound is made, a doctor dwells in the probe to observe organs that remain otherwise unseen: "It is not by looking at things (particulars), but by dwelling in them that we understand their joint meaning" (Polanyi 1966, p. 20). Because I have characterized the 'picture' doctors develop of their patients as an epistemic tool, we can understand the use of these tools in a similar fashion. The use of epistemic tools or theories become part of our subsidiary awareness, it is 'interiorized' to understand something, extending the cognitive apparatus of the knower. It is, however, important to note that subsidiary awareness is not necessarily unconscious awareness, because the use of the tool, such as the ultrasound apparatus or the epistemic tool, can always be brought back to the focal awareness, for instance when inspecting its proper functioning or adequateness, or, in the case of the epistemic tool, when new information becomes available. 
But, this is at the cost of focal awareness of the observed object. When one draws their attention away from the focal attention to the subsidiaries, the conception of the entity which is recognized or understood through the subsidiary use of technical or epistemic tools - is destroyed, in the same way as a pianist who, when turning her attention from the music to the separate notes and the movement of her fingers, will likely become confused and cease to play (ibid, p. 73). In other words, part of the 'triad' will remain unspecifiable at a specific moment in time, as it is impossible to focus one's direct attention on it. In summary, Polanyi recognizes that although during the act of knowing it is impossible to articulate which particulars make up the background clues of the subsidiary awareness, it is very well possible to make a reconstruction (although this is usually not a complete account of how knowledge is perceived). Furthermore, Polanyi explicates the relationship between what is tacitly known and what is consciously known.

I will now apply Polanyi's original conception of tacit knowing to analyse the skilful execution of epistemic activities in clinical decision-making that I have listed in the section epistemic activities. As mentioned in that section, I consider clinical decision-making as a system of practice consisting of three epistemic activities that each consist of several component activities. First, the gathering and critical assessment of relevant information, second the construction of a coherent 'picture' of the individual patient from these heterogeneous pieces of information, and third the application and adaptation of this 'picture'. Being competent in performing these specific epistemic activities is an important aspect of the expertise of doctors, which allows them to face the main epistemological challenges of clinical practice responsibly. In the following paragraphs, I will explain these epistemic activities as an interplay between subsidiary and focal awareness and thereby indicate how practitioners can be held accountable for how well they perform epistemic activities in decision-making.

\subsubsection{Gathering and assessment of information}

Physicians need to gather relevant information about the patient by taking the patient's medical history, performing physical examination of the patient, by ordering lab tests and images and searching the literature or textbooks. In all of these situations, the relevance and utility of the information need to be assessed - for example in the case of a literature search. As the clinical encounter usually commences with the question 'what brings you here today?' (Montgomery 2006), the first set of information that doctors collect is a patient's account of their symptoms. When doctors attend to their patients to gather information through a physical exam and medical history taking, theoretical information functions as subsidiary awareness, as background information about possible diagnoses and pathophysiological mechanism that cause the symptoms, from which clinicians direct 
their focal awareness to the conversation with the patient and the proceedings of the exam. As such, the subsidiary information functions as a conceptual and theoretical framework that allows coming up with and asking the right questions, interpreting the answers, and subsequently following up on them. The gathering of particular patient information thus requires the subsidiary use of theoretical knowledge whilst directing one's focal awareness to perform epistemic activities such as prioritizing certain questions and interpreting answers in light of prior knowledge. Conversely, while searching the scientific literature and textbooks, the particular signs and symptoms of a patient function as tacit knowledge, residing in a clinicians' subsidiary awareness, from which clinicians are able to direct their focal awareness, to the question to be answered, thereby assessing the relevance of the information that is coming across. In short, deciding on what information to use in a particular case, which is an epistemic activity, relies on the tacit use of knowledge of particular aspects of a patient.

As Collins and Evans contend, the subsidiary information that experts use tacitly is often selected unselfconsciously. However, it is a mistake to conclude, as they seem to suggest, that subsidiary information cannot be identified and evaluated and that choices that were made unselfconsciously are unjustifiable. It is possible to check, for instance, whether the theoretical information used in clinical decisions was up-to-date and relevant for the specific case at hand. Examples of such inquiries are: Is the information based on good quality science, and if such scientific support is not available what else warrants the use of that piece of information? When the patient's story is subsidiary, which information is given priority and for what reason? Did the physician obtain the story first-hand? Is the patient considered to be a trustworthy source? Did the patient provide enough information? These examples illustrate that, although knowledge is used tacitly and involves a considerable amount of choice and variability, physicians can be held accountable for the quality of knowledge they use and how they use it.

\subsubsection{Constructing the epistemic tool in the sense of a 'picture' of the patient.}

In order to construct a useful epistemic tool, (i.e., the 'picture' of an individual patient) it is necessary that the information gathered by a clinician - the written, scientific or general knowledge as well as patient-specific evidence such as lab results, imaging and clinical history - is comprehensive, relevant and up-to-date. Subsequently, the construction and use of such an epistemic tool involves tacit knowledge and epistemic activities. For example, in fitting the different pieces of information together, these pieces need to be weighted and mutually adjusted, for example by interpreting measurements in light of scientific theories or possible diagnoses, contextualizing theoretical knowledge and drawing creative relationships between different types of information. This requires epistemological 
choices and an active involvement of the epistemic agent. Tacitly, thus in their subsidiary awareness, prior experience with similar patients is used to draw analogies with the current patient. Through the subsidiary awareness of biomedical knowledge, the relevance of particular signs or symptoms can be assessed and weighted in relation to others. By subsidiary awareness of results from earlier tests, the results of new tests can be interpreted and fitted in with what is already known. When a disease develops over time, an up-todate picture of a patient is obtained through subsidiary awareness of the prior instances of the disease in that patient. Such uses of tacit knowledge allow doctors to fit together relevant information (the 'particulars') according to epistemic criteria, such as internal logical consistency, coherence with background knowledge, and comprehensiveness of information, thus directing their focal awareness to the 'whole,' the resulting 'picture' that is under construction.

\subsubsection{The adaptation and application of a 'picture' of the patient.}

The resulting 'picture' functions as an epistemic tool that allows epistemic actions, such as further reasoning, hypothesizing, and making diagnostic and treatment decisions. ${ }^{17}$ In these cases, the epistemic tool, similarly to physical tools, is used tacitly, focusing the attention on the goal of performing epistemic activities (e.g., to draw up a possible diagnosis and treatment plan). In Polanyi's words, talking about physical tools in quite similar ways as Collins and Evans, "we are attending to the meaning of its impact on our hands in terms of its [the physical tool's] effect on the things to which we are applying it" (Polanyi 1966, p. 13). The use of such a tool requires skills. Mastering cognitive skills in clinical practice is in that sense on the same footing as physical skills, as also recognized by Polanyi: "The art of the expert diagnostician [...] we may put it in the same class as the performance of skills, whether artistic, athletic or technical," (ibid, p. 6). In other words, the cognitive skills that enable a medical expert to perform diagnosis are similar to the skills of painters handling their brush and paint, a football player skilfully passing an opponent with a ball, or a surgeon closing a wound with sutures. And, similar to other skills,

17 It is important to note that an epistemic tool is a "tool in its own making" (Knuutila and Boon, 2011, p. 318): as it is being used to formulate questions and hypotheses it is adapted to newly gained insights. In other words, it develops through its use, in an interaction between tacitly held knowledge, experience, explicit reasoning, in consultation with others and by including new information. In addition, the epistemic criteria mentioned above (consistency, coherence, specificity and comprehensiveness) remain continuously relevant, both in the construction and use of an epistemic tool. Thus, although the epistemic activities differ between construction and use of an epistemic tool, a distinction between the two are analytical rather than being two consecutive, distinct steps in the diagnostic process. 
different levels of mastery can be obtained, from novice to expert level, through practice and experience. Analogous to the responsibility of clinicians to ensure their competence in physical skills, ${ }^{18}$ doctors have a responsibility to develop and cultivate their cognitive skills, which is part of their epistemological responsibility.

Finally, Polanyi's notion of tacit knowledge enables us to better understand two other important aspects of medical expertise playing a role in the three epistemic activities just listed, which set experts apart from novices. First, the ability of experts to deploy large amounts of information, including text-book knowledge and experience in their subsidiary awareness while focusing their attention on the patient-at-hand. When faced with cognitive tasks, a range of earlier acquired knowledge and information is tacitly invoked through which experts focus on the epistemic action. In contrast, novices still need to direct their focal attention to particulars such as theoretical information while talking to the patient or focus on patient particularities while searching for information. Analogous to the piano player who becomes confused when focusing on separate notes or finger movements, this focusing of attention impedes the execution of epistemic actions. Thus, similar to the mastery of physical skills, doctors and other experts master cognitive skills through practising and improving their ability to perform epistemic activities, being able to include more and more knowledge tacitly while being focused on the specific action.

A second aspect that sets experts apart from novices is the tacit recognition of relevant patterns. By having gained experience from diagnosing and treating many patients, expert clinicians have collected a wide range of exemplary cases including signs and symptoms, the diagnosis that followed, the choice of treatment and the clinical outcome. Clinicians are able to group these cases together according to similarities and differences between cases and distil what combination of signs and symptoms, measurements and other evidence suggests that a case belongs to a certain group. In interaction with patients or evidence, these patterns enable to recognize possible diseases and direct courses of action. Tacit pattern recognition can be compared to chess grandmasters - an example used by Polanyi - who recognise patterns in the way the pieces are positioned on the board without having to assess the position and possible course of each individual piece, and who can act accordingly. Such a catalogue of patterns is build up through practice and

18 In the Dutch health care law doctors carry a personal responsibility to develop and cultivate their competence in medical procedures ('geneeskundige handelingen') that are reserved to be solely performed by professional clinicians. 
from prior experience..$^{19}$ Important to our argument is that, very similar to theoretical knowledge acquired in formal education, this catalogue of patterns operates as subsidiary awareness, allowing physicians (and chess grandmasters) to recognize what is going on and how to handle it. This is not to say that pattern recognition is a justification for a medical decision at the same level as theoretical and evidence-based knowledge. Instead, it is an indispensable mechanism that fills the gaps not yet covered by formally-tested knowledge. Furthermore, pattern recognition enables physicians to direct their reasoning and search for further information. Although patterns are recognized tacitly, instantly and unselfconsciously, they are open to evaluation and physicians are responsible for the conclusions they reach on the basis of a recognized pattern.

\subsection{The epistemological responsibility of experts in clinical deci- sion-making}

One of the main criticisms against the epistemology of EBM is that it wrongly assumes that the role of expertise in medical decision-making can and should be reduced in order to increase objectivity and quality. Nevertheless, EBM has rightfully challenged "authority-based" decision-making, by demanding quality in accordance with scientific standards. In this chapter, I have reconciled EBM's call for quality decision-making with medical expertise. I have argued that what seems to be missing in current accounts of medical expertise is an understanding of the role of the systematic reasoning in medical decision-making about individual patients. Therefore, I have argued that a more detailed analysis of what medical expertise entails is needed. I have first turned to Collins and Evans, who have highlighted that expertise is about what experts can do rather than primarily about what they know, and who have pointed out that tacit knowledge is a central trait of experts. In addition to their analysis, which focuses on cultural integration, mastering physical skills and epistemic content, I have referred to Hasok Chang's account of epistemic activities and argued that an understanding of expertise must also include the role of cognitive skills that enables experts to perform certain epistemic activities. Furthermore, it should include an explanation of how tacit knowledge allows experts to

19 Donald Schön (1983) characterizes this process as 'seeing as': a professional sees a specific situation as another, recognizing that it is both similar in some ways and at the same time different in other ways (hence, they do something different from seeing it as an instance of a general category or law). To do this, practitioners build up a repertoire of examples, images, understanding and actions from experience. See Chapter 1. 
practice their expertise. Michael Polanyi's original notion of tacit knowledge is richer and more detailed than that usually put forward by authors defending medical expertise. Polanyi describes tacit knowing as a triad consisting of three components, the knower, their subsidiary awareness, and their focal awareness. This triadic conception allows for a more fine-grained analysis of the epistemic actions that make up decision-making for diagnosis and treatment, and of how tacit knowledge is a specific trait of experts in comparison to novices.

An important result of elucidating the role of tacit knowledge in expertise is that it clarifies how medical experts can be held accountable for epistemic activities in medical decision-making. I have argued that cognitive skills and competence in epistemic activities are considered to be crucial aspects of medical expertise and doctors have a responsibility to develop, acquire and cultivate these skills and competencies in a similar way as they have a responsibility to develop, acquire and cultivate physical skills such as surgical skills. I follow Collins and Evans in their claim that tacit knowledge plays a pivotal role in expertise, but I refuse to regard it as inarticulate and therefore hidden from evaluation. Rather, by referring to Polanyi's original conception of tacit knowing, I showed that there is a distinction between subsidiary and focal attention and that knowledge in one's subsidiary awareness can be opened up to scrutiny, so that doctors can be held accountable for what information is used tacitly, be it textbook knowledge, outcomes from RCTs or experience. What information is used, in either one's subsidiary or focal awareness, should be justifiable by the knower. By giving an account of how medical experts employ tacit knowledge and how they become skilled in performing epistemic activities, I have detailed what the epistemological responsibility of doctors entails.

The debate about the role of the expert versus EBM in decision-making reflects a similar debate in the philosophy of science. Similar to EBM advocates, philosophers of science seem to fear that the dismissal of the idea of objective, detached knowledge paves the way for subjectivism or relativism. Each of the authors quoted in the introduction, however, emphasize that an approach that assigns epistemic agents a central role allows a better evaluation of epistemic products than dogmatic adherence to general concepts such as truth, reality and objectivity. Chang, for instance argues that focus on epistemic activities allows normative evaluation, assessing the criteria scientists use to evaluate their own and each other's activities (for instance, truthfulness or relevance). It also allows external agents to make independent judgements of scientific research practice that take the complexities and specificities of the practice into account. De Regt stresses that truth is not the main criterion to evaluate the quality of scientific models or theories. Rather, in order to facilitate understanding, scientific theories or models should be truthful with respect to the 
empirical phenomena that they model or describe, depending on the aims of the knower, as well as intelligible, depending on the skills of the knower. In addition, De Regt argues that non-objective elements (such as skills and aims) do not necessarily mean subjective: "Skills are acquired within a community and assessed by that community. This guarantees that judgments based on skills are not arbitrary" (De Regt 2009, p. 37). Boon (2017b) mentions several epistemic criteria to evaluate epistemic tools. ${ }^{20}$ Some of these criteria refer to impersonal characteristics, such as empirical adequacy, logical consistency and coherence with accepted knowledge, but many others refer to epistemic use and the user such as relevance in view of the epistemic purposes, simplicity and validation in view of epistemic uses. In short, these authors emphasize that non-objective does not have to be the same as subjective. In contrast, more attention to epistemic agents and epistemic use allows for a more adequate evaluation, in the sense of better fitting the practice of science. The framework of expertise that I present here gives guidance to how judgments made on the basis of skills can be evaluated as well, responding to the fear of relativism that is evoked by accounts that emphasize the role of the knower in epistemology.

In conclusion, a crucial aspect of (medical) expertise is competence in performing epistemic activities, which requires cognitive skills and, similar to physical skills, mastering cognitive skills entails internalisation or tacit knowledge. Doctors can and should be held accountable for how well they perform epistemic activities in decision-making. This means that they can be held responsible for acquiring, developing and maintaining competence in cognitive skills, and also that they bear responsibility for the quality of the knowledge and reasoning processes. This is what I have called their 'epistemological responsibility'.

20 These are: empirical adequacy, reliability and relevance in view of epistemic purposes; simplicity in the sense of manageability \& tractability; balance between generality \& specific in view of epistemic aims; explanatory \& predictive reliability; logical consistency; coherence with accepted knowledge relevant to epistemic uses; integration of knowledge from different fields and levels; validation in view of epistemic uses. 


\section{References}

Boon, M. (2017a), 'Philosophy of science in practice: A proposal for epistemological constructivism', in H. Leitgeb, et al. (eds.), Logic, Methodology and Philosophy of Science - Proceedings of the 15th International Congress (CLMPS 2015) (College publications), 289-310 ISBN: 978-1-84890-229-9

Boon, M. (2017b), 'An engineering paradigm in the biomedical sciences: Knowledge as epistemic tool', Progress in Biophysics and Molecular Biology, 129, 25-39 DOI: 10.1016/j.pbiomolbio.2017.04.001.

Boon, M. and Knuuttila, T. (2009), 'Models as epistemic tools in engineering sciences: a pragmatic approach', in A. Meijers and Dov M. Gabbay (eds.), Philosophy of Technology an Engineering Sciences (Handbook of the philosophy of science; North Holland: Elsevier Science \& Technol), 687-720 ISBN: 978-0-444-51667-1

Chang, H. (2009), 'Ontological principles and the intelligibility of epistemic activities.', in H. W. De Regt, S. Leonelli, and K. Eigner (eds.), Ontological principles and the intelligibility of epistemic activities (Pittsburgh: Ontological principles and the intelligibility of epistemic activities) ISBN: 9780822971245

Chang, H. (2012), Is Water H2O? - Evidence, Realism and Pluralism (Boston Studies in the Philosophy and History of Science; Dordrecht: Springer) ISBN: 978-94-007-3931-4.

Chang, H. (2014), 'Epistemic Activities and Systems of Practice: Units of Analysis in Philosophy of Science After the Practice Turn.', in L. Soler, et al. (eds.), Science After the Practice Turn in the Philosophy, History and Social Studies of Science. (London: Routledge), 67-79 ISBN: 978-0415722957

Code, L. (1984), 'Toward a 'Responsibilist' Epistemology', Philosophy and Phenomenological Research, 45 (1), 29-50 DOI: 10.2307/2107325.

Collins, H. and Evans, R. (2007), Rethinking Expertise (Chicago, London: The University of Chicago Press) ISBN: 978-0-226-11360-9.

Collins, H. M. (2001), 'Tacit Knowledge, Trust and the Q of Sapphire', Social Studies of Science, 31 (1), 71-85 DOI: $10.1177 / 030631201031001004$.

de Regt, H. W. (2015), 'Scientific understanding: truth or dare?', Synthese, 192 (12), 3781-97 DOI: 10.1007/ s11229-014-0538-7.

Dreyfus, H.L., Dreyfus, S. E., and Athanasiou, T. (1986), Mind over machine: the power of human intuition and expertise in the era of the computer (The Free Press) 231 ISBN: 0-02-908060-6.

Goldman, Alvin I. (2016), 'Expertise’, Topoi, 1-8 DOI: 10.1007/s11245-016-9410-3.

Greenhalgh, T. et al. (2014), 'Evidence based medicine: a movement in crisis?', BMJ, 348, g3725 DOI: 10.1136/ bmj.g3725.

Guyatt, G. H. et al. (1992), 'Evidence-based medicine: A new approach to teaching the practice of medicine', JAMA, 268 (17), 2420-25 DOI: 10.1001/jama.1992.03490170092032.

Hempel, C.G. (1965), Aspects of scientific explanation (New York: Free Press) ISBN: 0029143403

Henry, S. G. (2010), 'Polanyi's tacit knowing and the relevance of epistemology to clinical medicine', J Eval Clin Pract, 16 (2), 292-7 DOI: 10.1111/j.1365-2753.2010.01387.x.

Knuuttila, T. and Boon, M. (2011), 'How do models give us knowledge? The case of Carnot's ideal heat engine', European Journal for Philosophy of Science, 1 (3), 309-34 DOI: 10.1007/s13194-011-0029-3.

Loughlin, M. (2008), 'Reason, reality and objectivity--shared dogmas and distortions in the way both 'scientistic' and 'postmodern' commentators frame the EBM debate', J Eval Clin Pract, 14 (5), 665-71 DOI: 10.1111/j.1365-2753.2008.01075.x.

Loughlin, M. (2009), 'The basis of medical knowledge: judgement, objectivity and the history of ideas', J Eval Clin Pract, 15 (6), 935-40 DOI: 10.1111/j.1365-2753.2009.01318.x. 
Loughlin, M. (2010), 'Epistemology, biology and mysticism: comments on 'Polanyi's tacit knowledge and the relevance of epistemology to clinical medicine", J Eval Clin Pract, 16 (2), 298-300 DOI: 10.1111/j.13652753.2010.01388.x.

Malterud, K. (2001), 'The art and science of clinical knowledge: evidence beyond measures and numbers', The Lancet, 358 (9279), 397-400 DOI: 10.1016/S0140-6736(01)05548-9.

Mitchell, M.T. (2006), Michael Polanyi: The art of knowing, ed. J. Beer (Library of modern thinkers; Wilmington, Delaware: ISI books) ISBN: 978-1932236903.

Montgomery, K (2006), How Doctors Think: Clinical Judgment and the practice of medicine (New York: Oxford University Press) ISBN: 9780195187120.

Polanyi, M (1958), Personal Knowledge: Towards a Post-Critical Philosophy (1974 edn.; Chicago and London: The University of Chicago Press) ISBN: 041515149X.

Polanyi, M (1966), The Tacit Dimension (Chicago and London: The University of Chicago Press) ISBN: 9780226672984 .

Schön, D. (1983), The Reflective Practitioner - How professionals think in action. (New York: Taylor \& Francis Ltd.) ISBN: 978-0465068784.

Tanenbaum, S. J. (1993), 'What Physicians Know', New England Journal of Medicine, 329 (17), 1268-71 DOI: 10.1056/nejm199310213291713.

Tonelli, M. R. (1998), 'The philosophical limits of evidence-based medicine', Academic Medicine, 73 (12), 1234-40

Tonelli, M. R. (2006), 'Evidence Based Medicine and Clinical Expertise', Virtual Mentor: Ethics Journal of the American Medical Association, 8 (2), 71-74 DOI: 10.1001/virtualmentor.2006.8.2.ccas1-0602.

Worrall, J. (2002), 'What Evidence in Evidence-Based Medicine? ', Philosophy of Science, 69 (S3), S316-S30 DOI: $10.1086 / 341855$.

Wyer, P. C. and Silva, S. A. (2009), 'Where is the wisdom? I--a conceptual history of evidence-based medicine', J Eval Clin Pract, 15 (6), 891-8 DOI: 10.1111/j.1365-2753.2009.01323.x. 


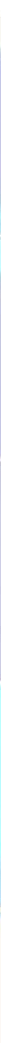




\section{Disciplinary perspectives and interdisciplinary research, a problem-oriented account}

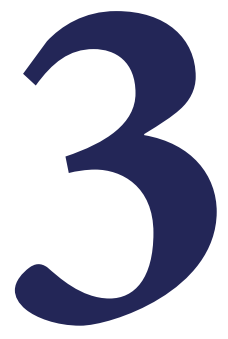

An adapted version of this chapter is accepted for publication as: M. Boon, S. van Baalen and M. Groenier (Forthcoming) Interdisciplinary expertise in medical practice: Challenges of using and producing knowledge in complex problem-solving. Medical Teacher

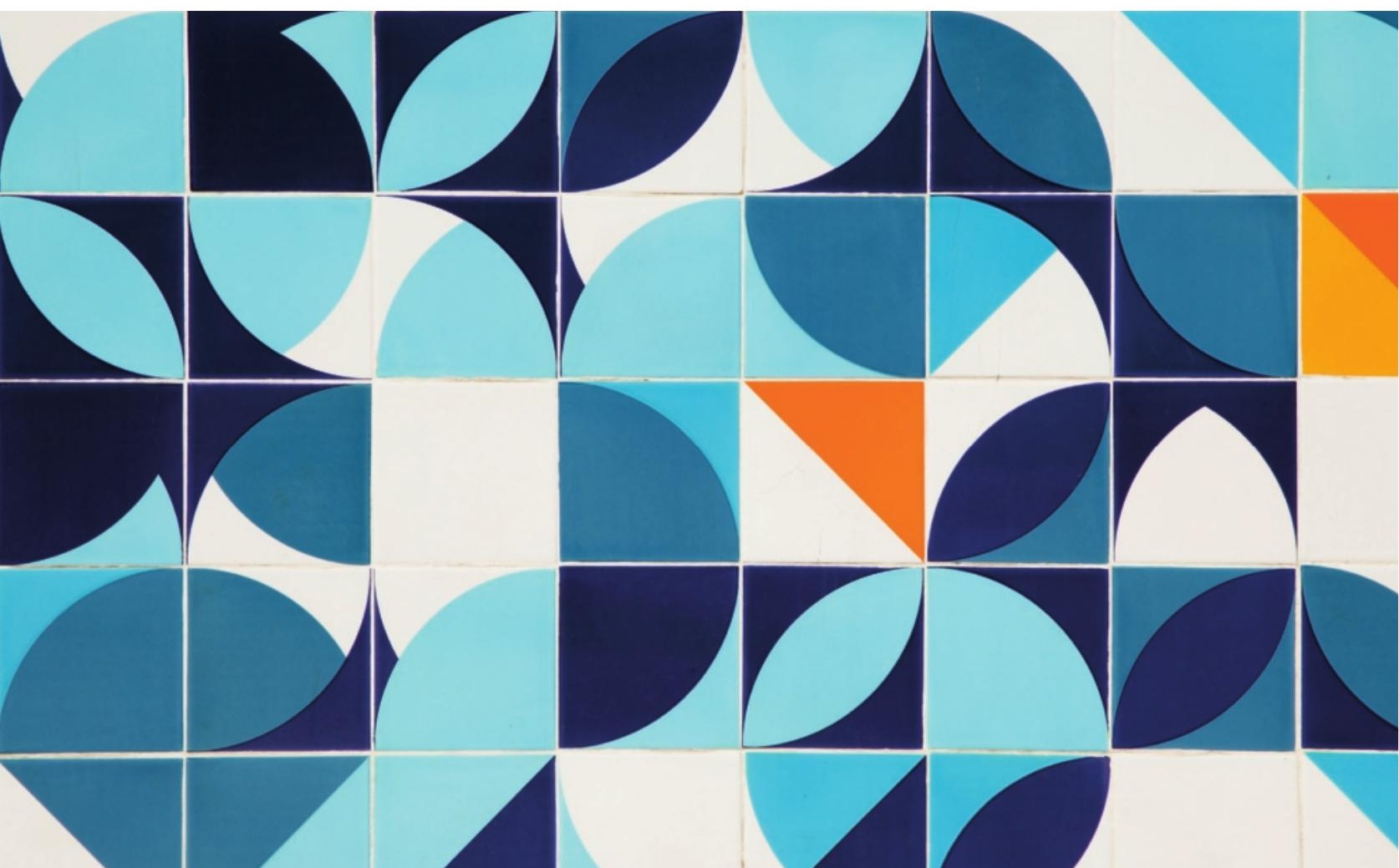




\section{Abstract}

Expertise, according to Fleck, Kuhn, and Collins and Evans, is obtained or learned by being immersed in a scientific community. I will argue that this implies that experts develop a disciplinary perspective that shapes how they deal with a target system. I view disciplinary perspectives as coherent sets of aspects such as goals, phenomena of interest, objects of investigation, ways of modelling, theories and concepts, methodology and instruments, and epistemic values and scientific ideals. In this chapter, I analyse an interdisciplinary research project in technical medicine to illustrate what I mean by 'disciplinary perspectives' and how disciplinary perspectives shape the construction of knowledge about a target system. Based on this analysis, I argue that interdisciplinary research aimed at problems in professional practices (also called 'real-world' problems) is not primarily about the integration of theories or concepts. Instead, I present a 'problem-oriented' account of interdisciplinary research, in which multiple disciplinary experts who each 'exercise' their own disciplinary perspective in dealing with aspects of the target system interact around a problem. Rather than aiming at integration of theories or concepts, interaction means that the experts, each having their own disciplinary perspective and thereby having their own take on the target system in regard of the aspects mentioned exchange epistemic results. This means that reasoning from one perspective provides input in another. Coming up with an interdisciplinary solution, therefore, involves going back and forth between multiple disciplinary perspectives in order to use them in reasoning about the target system and effectively combine them in a solution that is internally coherent as well as consistent with all perspectives involved. When disciplinary experts are engaged in interdisciplinary research collaborations they need specific cognitive abilities that are not automatically learned when someone becomes an expert within a specific discipline. Therefore, I argue that for interdisciplinary research, researchers should obtain interdisciplinary expertise: the cognitive ability to connect, translate, and establish links between disciplinary knowledge of the target system, by taking into account that knowledge is generated by disciplinary perspectives. I argue that this involves higher-order cognitive skills at two levels: the first level concerns the ability to use multiple disciplinary perspectives to reason about a target system whereas the second concerns the ability to reflect on multiple disciplinary perspectives to identify their differences and similarities in order to identify where gaps, opportunities and conflicts might arise and how this can be resolved into a solution that is at least acceptable to each discipline involved. Finally, I turn to technical medicine, an educational program that aims to train interdisciplinary professionals. I argue that the expertise of technical physicians is not fundamentally different from the interdisciplinary expertise of disciplinary researchers engaged in interdisciplinary, but more advanced. Technical physicians are specialized in solving a certain 
type of interdisciplinary problems (medical problems that require technological solutions) and are familiar with the disciplines involved to such an extent that they can take the role of 'connector, facilitating profound interactions between and contributions by disciplinary experts, which might not succeed if left to disciplinary experts themselves.

\subsection{Introduction}

One of the central claims in this dissertation is that knowers play an active and indelible role in the construction and use of knowledge and that to evaluate the quality of knowledge in practice we should turn our attention to what experts do when they construct and use knowledge in practice. In Chapter 1 and 2 I focused on clinical practice and outlined what attending to experts and what they do implies for clinical decision-making. In Chapter $1 \mathrm{I}$ argued that instead of deferring to clinical guidelines - in which scientific knowledge is evaluated according to rigid rules preferring certain methods over others - clinicians have an epistemological responsibility for making sound and well-informed decisions, based on all relevant and reliable information available. In Chapter 2 I argued that understanding this epistemological responsibility requires a closer examination of medical expertise and that having expertise involves having (cognitive) abilities to perform epistemic activities. In this chapter, I will argue that these abilities are shaped by a disciplinary perspective. To illustrate this, I will leave clinical decision-making for another practice related to medicine: the development of a clinical imaging tool, in which multiple disciplinary perspectives are involved. In addition, I will argue that a more comprehensive understanding of interdisciplinary research follows from this account. In my account, interdisciplinary research does not necessarily involve the integration of theories and concepts from different disciplines. Rather, disciplinary perspectives interact around a problem concerning a specific target-system, each contributing to a solution that is internally coherent and consistent with each discipline involved. This requires the ability to perform specific epistemic activities, which involves interdisciplinary expertise. I will explicate what I mean with this type of expertise and touch upon how it can be taught by referring to a new educational program training interdisciplinary professionals - technical medicine.

In Chapter 2 I refer to Collins and Evans' (2007) skills-based conception of expertise. In their view, to be an expert means having tacit knowledge: "tacit knowledge is the deep understanding one can only gain through social immersion in groups who possess it," (Collins \& Evans 2007, p. 6). In other words, expertise, the required skills and (tacit) knowledge are rooted in certain groups or communities in which relevant knowledge, 
methods and epistemic values are shared. In addition, institutional elements facilitate the internal cohesion of these groups. Within academia and medicine, such groups are usually called 'disciplines'. In this chapter, I accept Collins and Evans' view that being an expert implicates belonging to a certain group, and that being an expert means being an expert in something. However, where Collins and Evans' analysis focuses on the social embedding of experts within groups, I will focus on the epistemological underpinnings and implications of belonging to a discipline. My starting point is the idea that methods, ways of measuring, reasoning and modelling are defined, shared and learned within a discipline (Collins and Evans 2007; Fleck 1979; Kuhn 1970) and that this shapes how knowledge is constructed and used (MacLeod 2018).

In addition to the content of a discipline (e.g. theories and concepts) and procedural aspects (e.g. methodology and ways of modelling) a disciplinary perspective also entails normative and metaphysical aspects, guiding how epistemic activities are performed and evaluated. Authors whom I have discussed in the previous chapters point to such aspects. Steven Toulmin (2001) describes professional practices in which scientific knowledge is used to solve real-world problems as follows:

"These practices begin with intelligent analyses of the factual soil from which our problem spring, but such actions bear fruit only when they are guided by ideals that make rational assessments stepping stones to reasonable decision. In these terms, a doctor skilled in molecular biochemistry alone is not the kind of professional the future calls for, any more than an engineer who knows only how to compute the size of girders capable of providing a given strength, or an economist who knows only how to calculate the rates of interest needed to maintain a desired return on investment," (Toulmin 2001, p. 213).

In other words, professional practices are not only a matter of rational deliberation that is informed by results from scientific research but also by a set of (epistemic) values and ideas about the aims of the practice that guide judgments and decisions. Schön (1983) makes a similar argument, saying that when practitioners frame a situation as another (in terms of their differences and similarities, see Chapter 1 for more details) they perform a kind of thought experiment on the situation, exploring the implications and unexpected consequences of framing that situation in that particular way. Through a sequence of these attempts to frame and change the situation, it becomes understood by the practitioner. The evaluation, and eventually accepting, of a specific way of framing is, according to Schön, grounded in a practitioner's appreciative system (Schön 1983). Khushf (1999) refers to similar conceptual frameworks to explain how practitioners assess the judgments based on scientific knowledge in practical situations, but argues that these frameworks are rooted 
in (scientific) communities. Khushf argues that the 'feeling of accord' (a feeling that a concept is 'right' or 'fitting') that leads to accepting the purposiveness of a preliminary formed diagnosis (an as-of-yet unspecified universal that goes up for testing) is guided by a scientific ideal. According to Khushf, this ideal is a meta-theoretical understanding of the practice within which a judgment is made as well as of its ends, which is the result of the historical development of science (Khushf 1999).

In short, according to these authors, scientists and professionals are guided by scientific ideals concerning the aims of their work as well as (epistemic) values and metaphysical background pictures that guide what problems they consider relevant and how solutions are judged. In other words, the performance of epistemic activities is guided by a conceptual framework that finds its roots in collectives. Together, the content, the normative aspects and the procedural aspects of the shared conceptual framework form a coherent whole, which, metaphorically speaking is the disciplinary perspective through which experts look, think, interpret and explain, in other words, perform epistemic activities, often without being aware of 'having a perspective'. In the following paragraph, I will further elaborate on the disciplinary perspectives and their role in practice. After that I will present an example case concerning the development of a clinical imaging tool, in which multiple disciplinary perspectives are involved, to illustrate what I mean by 'disciplinary perspectives.' Then, I will argue that this view has implications for our understanding of interdisciplinary work. I will argue that interdisciplinary work does not necessarily mean integration of theories or concepts from disciplines. Rather, it involves the interaction of disciplines around a problem concerning a target system, in which professionals with different disciplinary perspective exchange epistemic results. Finally, I will argue that this type of work requires interdisciplinary expertise and will explain in more detail what this type of expertise entails and how it can be taught in the Paragraphs 3.7 and 3.8.

\subsection{Disciplinary perspectives}

My primary claim for this chapter is that 'knowledge' of a specific target-system is generated by an expert within a specific discipline or specialism. Within this discipline, scientific ideals, epistemic values as well as specific ways of measuring, reasoning and modelling shape how an expert 'sees' a certain target-system. This collection of aspects typical of a 
discipline or specialism forms a disciplinary perspective. ${ }^{21}$ This idea can be traced back to Kant, who argued that the world does not speak for itself - or in other words that objective knowledge is not generated by observing phenomena that create an unaltered imprint in our mind (in contrast to what is believed in positivist or empiricist philosophy, or what Boon (2017) calls the 'representational view' of science). Instead, the human cognitive system plays an inherent and indelible role in generating knowledge from experiences of the world, by constructing an explanation of these experiences by structuring and conceptualizing observed phenomena. According to Kant, to do this the mind employs regulative principles. ${ }^{22}$ Differently said, people inevitably employ and invent all kinds of concepts to interpret what they 'see.' Examples are basic concepts such as 'time,' 'space,' 'object,' 'property,' 'process,' 'causes' and 'interaction.' Less abstract are concepts used in counting and comparing, in quantifying and qualifying (e.g. size, weight, smaller, bigger, heavier). Similarly, there are the concepts in logic, mathematics and science used to generate knowledge about the world. According to Kant, these concepts are not a property of the world independent from human cognition. Instead, they sprout from the human mind. Without cognitive activities, i.e. without inventing and employing concepts to interpret, structure and systematize information, there is no knowledge - not even at the most basic level of immediate experiences (Boon 2017).

An early philosopher of science who connected thinking to groups or communities is Ludwig Fleck. Fleck (1979) refers to thought styles and thought collectives to describe how experts from a certain (professional or academic) community adopt similar ways of

21 Ronald Giere (2006) has put forward an account involving perspectives in his book scientific perspectivism. He argues that both scientific observations and theories are perspectival, that these perspectives are partial and of limited accuracy, that scientific claims apply to the fittingness of a perspective and that representation is a relation between theory, world, actor and purpose: 'S uses $\mathrm{X}$ to represent $\mathrm{W}$ for purposes $\mathrm{P}$, (Giere 2006, p. 60). In this chapter, rather than claiming a perspectival nature of scientific representations, I argue that disciplines have a perspectival character. There are, nevertheless, several similarities between my notion of disciplinary perspectives and Giere's notion of perspectivism: disciplinary perspectives are partial and of limited accuracy, multiple disciplinary perspectives form a "plurality of overlapping perspectives, which are compatible in one sense, which are all perspectives on the same world [or target system], but don't add up to an absolute view of the world," (Brown 2009, p 219).

22 Boon (2017) mentions: the principle of induction; the principle that every event has a cause; the principle that nature forms a system according to laws; and the principle of the unity of nature (p. 299). Chang (2009) lists ontological principles that make epistemic activities intelligible. For example counting involves the principle of discreteness, prediction involves uniform consequences and explanation. 
perceiving and thinking. Fleck argues that there are three factors involved in cognition, i.e., the individual, the thought collective, and objective reality. Thought styles originate within a certain community and guides what problems members of that communities find relevant and how they approach these problems. "It is characterized by common features in the problems of interest to a thought collective, by the judgment which the thought collective considers evident, and by the methods which it applies as a means of cognition," (Fleck 1979 p.99). In short, the thought style determines the problems members of collective are interested in and how they approach investigating these problems, while at the same time it is also a disposition to overlook certain aspects. Fleck influenced the better-known work of Thomas S. Kuhn, and his central concept paradigms and scientific community are often erroneously equated to Fleck's thought styles and thought collectives (Mößner 2011). As Fleck, Kuhn considers knowledge to be deeply rooted in social communities, and both conclude that this defines the world the individual scientists inhabit.

In Kuhn's account, a paradigm is like a 'perspective' or a 'conceptual framework'; a background within which a scientific practice of a specific discipline is embedded, and which guides and enables that practice. Central to Kuhn's account is the notion of a paradigm shift (which is where Kuhn differs from Fleck, who envisions a more continuous change of thought styles). Revolutionary new theories could not have emerged or become accepted within the existing paradigm of their days - they involved a radical change. Over time, Kuhn's notion of paradigm has acquired a narrow and a wide meaning. In the Postscript to the second edition of The Structure of Scientific Revolutions, Kuhn (1970) aimed to specify the wider meaning of paradigm in terms of a disciplinary matrix. With regards to this term, Kuhn wrote: "For present purposes I suggest 'disciplinary matrix': 'disciplinary' because it refers to the common possession of the practitioners of a particular discipline; 'matrix' because it is composed of ordered elements of various sorts, each requiring further specification" (Kuhn 1970, 182). ${ }^{23}$ The first type of component is 'symbolic generalizations,' which refers to the theoretical content of the discipline such as its laws (providing a syntax of the field in formalized terms, i.e. $\mathrm{F}=\mathrm{ma}$, as well as expressions in ordinary language, i.e. "elements combine in constant proportion by weight"). The second type of component is the 'metaphysical part of paradigms,' which consists of the shared commitments to such beliefs as: 'all perceptible phenomena are due to the interaction of qualitatively neutral atoms in the void,' or, alternatively, 'to matter and force,' or 'to fields.' Importantly, Kuhn stresses that scientific models explaining the phenomena may be taken metaphysically (i.e.

23 In several texts, Kuhn stresses that he does not attempt an exhaustive list, but only notes the main sorts of components of a disciplinary matrix (e.g., 1970, 182). 
literally) or heuristically, as in cases where the model is clearly intended as an analogy, a 'seeing-as', such as in 'the molecules of a gas behave like tiny elastic billiard balls in random motion.' Kuhn describes the third sort of component as 'values' used to judge theories, rebutting the idea that 'truth' is the ultimate epistemic value by which theories are accepted or rejected. The fourth and last component that Kuhn (1970) points out, he calls 'exemplars', which aligns with the narrow view of paradigms. Kuhn described 'exemplars as "concrete problem solutions, accepted by the group as, in a quite usual sense, paradigmatic" (Kuhn 1977, p. 298). Exemplars are illustrations of the symbolic generalizations. They are examples of problem-solutions by means of which students, first, learn how to relate symbolic generalizations to problems (e.g., the inclined plane, the conical pendulum, and Keplerian orbits), and next, by learning to see new problems as similar to those of the exemplars, will be able to apply the problem-solution strategies illustrated by means of the exemplar to new situations. Following Kant, Fleck and Kuhn, I argue that knowledge does not speak for itself. Rather, it is guided by an experts' disciplinary perspective that consist of the basic theories and concepts that shape perception and observation and structure and systematize information, as well as the main goal of a discipline, the phenomena of interest, the objectives of investigation, the way(s) of modelling, the methodology and instruments and the scientific ideals and epistemic values.

In the following paragraph, I will illustrate the concept of 'disciplinary perspective' through an example of a research project that I have worked on myself as a researcher. The goal of this project is to develop a new clinical imaging tool, i.e., diffusion magnetic resonance imaging (diffusion MRI), to characterize the microstructural makeup of kidney tumours. The target system of this study is the combination of the MRI-machine, the software necessary to produce images, and the patient with a (suspected) kidney tumour, including the wider practice of care of these patients in which the tool under development should function. Diffusion MRI allows for visualization and quantification of water diffusion without administration of exogenous contrast materials and is, therefore, a promising technique for imaging kidney tumours. In earlier studies, several parameters derived from diffusion MRI studies were found to be able to differentiate between different tumour types found in the kidney (Chandarana et al. 2012; Feng et al. 2016; Rheinheimer et al. 2012). Imaging methods available in current clinical practice can detect the size and location of kidney tumours, but tumour type and malignancy can only be determined histologically after surgery. The purpose of the study is to assess whether other diffusion MRI derived parameters, that are obtained by fitting to more advanced diffusion models (van Baalen et al. 2017; van der Bel et al. 2017) can better differentiate between malignant and benign kidney tumours (Van Baalen et al. under review). Being able to make this distinction could potentially prevent unnecessary surgery in patients with non-malignant 
tumours. To develop a system that functions properly, multiple experts with different disciplinary perspective are involved: from MRI physics, MR diffusion-weighted imaging, image processing, (kidney) tissue physiology, and kidney tumour clinical care. I will now give a description of each of these disciplinary perspectives, first describing the field in general and then highlight some of the aspects of the disciplinary perspective. See Table 1 for an overview of these aspects for each disciplinary perspective.

\subsubsection{MRI physics}

Magnetic resonance imaging is based on the physics of magnetism and the interaction of tissue components with radio magnetic fields. The main component of the human body that clinical MRI machines are sensitive to is (the number of) water molecules or, more specifically, hydrogen nuclei (protons). These protons can be thought of as rotating or spinning, producing (tiny) magnetic fields. By placing tissue that contains protons in a relatively strong magnetic field (usually 1.5 or 3 Tesla emitted by a large coil that surrounds the body), the tiny magnetic fields of these protons will align themselves with the direction of the strong magnetic field. By then applying a series of radiofrequency pulses, protons will be pushed out of balance and rotate back to their original state, causing a magnetic flux that causes a change in voltage which is picked up by receiver coils in the MRI machine. The rate with which protons return to their original state, the relaxation time, is influenced by the makeup of their environment, and will, therefore, differ for different tissues, resulting in image contrasts between tissues. This property is often expressed as $\mathrm{T} 1$ or $\mathrm{T} 2$, which are different measures of relaxation time. To be able to form images of the signal, magnetic field gradients are applied, spatially varying the field, which enables to differentiate between signals from different locations. Computer software using mathematical formulas 'translate' the signal into a series of images. See figure 3.1 for a schematic overview of the MRI machine (A) and the proton spins (B). 


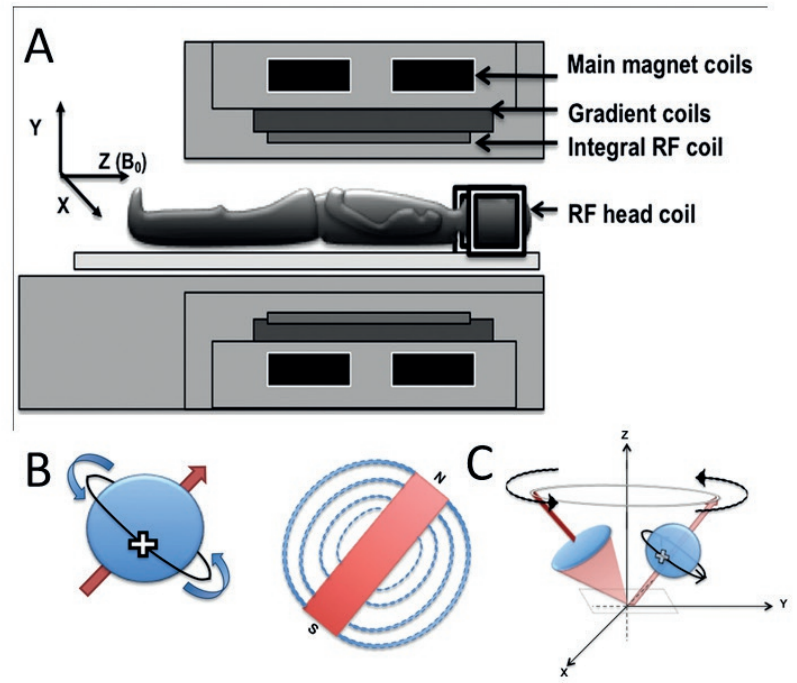

Figure 3.1: MRI physics. ${ }^{24}$

\subsubsection{The disciplinary perspective}

Using the elements of the matrix (i.e., goal, phenomena of interest, objects of investigation, way(s) of modelling, theories and concepts, methodology and instruments, epistemic values and scientific ideals), the disciplinary perspective of MRI physics can be specified as follows: The goal of MRI physics is to develop machines that induce and manipulate the nuclear magnetic resonance (NMR) signal and produce accurate representations of the object of imaging based on this signal. The phenomena that MRI physics deals with are magnetic fields, proton spins, radiofrequency pulses, relaxation times and coils (notably, not the (tumour)tissue that is imaged using MRI itself). The objects of investigation in this field involve developing new hardware (coils or other components) to improve image quality or to enable imaging of specific body areas, developing new acquisition schemes that allow new signal contrasts and hence visualizing new tissue aspects, and software development. The behaviour of the phenomena of interest and their interactions are modelled through mathematical equations, some of which stem from basic physics (i.e. those relating to magnetism), while others are more specific to MRI, (i.e., relax-

24 Reproduced from ['Understanding MRI: basic MR physics for physicians', Currie S, Hoggard N, Craven IJ, et al. Postgrad Med J; 89:209-223, copyright (2013)] with permission from BMJ Publishing Group Ltd 
ation times and formulas describing the relationship between the acquired signal and the image). Acquisition sequences are often visualized in a sort of block schemes that follow conventions from the field. Theories and concepts that are fundamental to this field are classical electrodynamics (describing (electro)magnetic fields, flux, induction, currents and coils), nuclear magnetic resonance (describing the magnetic moment of nuclei caused by resonance due to a magnetic field), relaxation and Fourier transform (fundamental to transforming the measured signal into images). Subfields are arranged according to acquisition method (e.g., diffusion MRI, functional MRI, etc.), body area or organ (e.g., abdomen, limbs, head, brain, heart) or pathology (e.g., tumours), or, more fundamental, hardware or software development. Methods of investigation in this field are, phantom experiments (to visualize or quantify known and controllable processes in MRI), experiments with healthy volunteers (to investigate the most adequate acquisition and modelling protocol for certain body areas), (clinical) trials with patient populations (to investigate the clinical value of a certain imaging protocol) and computer simulations (to simulate the effect of varying parameters). Practical constraints in this disciplinary perspective are formed by the physical MRI apparatus (i.e., bore size, magnetic field strength, gradient strength, etc.) that limits what objects can be imaged, the possible imaging parameters that can be obtained (i.e., field of view, signal to noise ratio, resolution, etc.) and the computing power of the computer with which data is transformed into images. The epistemic values of this disciplinary perspective are consistency between the theory, predictions and the phenomenon that is produced and measured, i.e., the MRI signal, reliability of production and manipulation of the MRI signal - which is often established in terms of reproducibility, similar results are obtained for similar cases - and validity of the accuracy of the images produced as representation for imaged object (i.e., a part of the human body).

\subsubsection{Diffusion MRI}

Diffusion MRI is a subfield of MR imaging, that is based on a specific way of obtaining the NMR signal. This is based on the random (Brownian) motion of water molecules in tissue. This motion is restricted by tissue components such as membranes and macromolecules and therefore water molecules move, or 'diffuse,' at different rates in different tissues, depending on the microstructure of tissues. The contrast between tissues is thus based on a different 'diffusion signal' in different tissues. To measure this, additional magnetic field gradients are applied which results in a signal attenuation proportional to the diffusion rate, as water molecules 'move' out of their original voxel due to diffusion. 

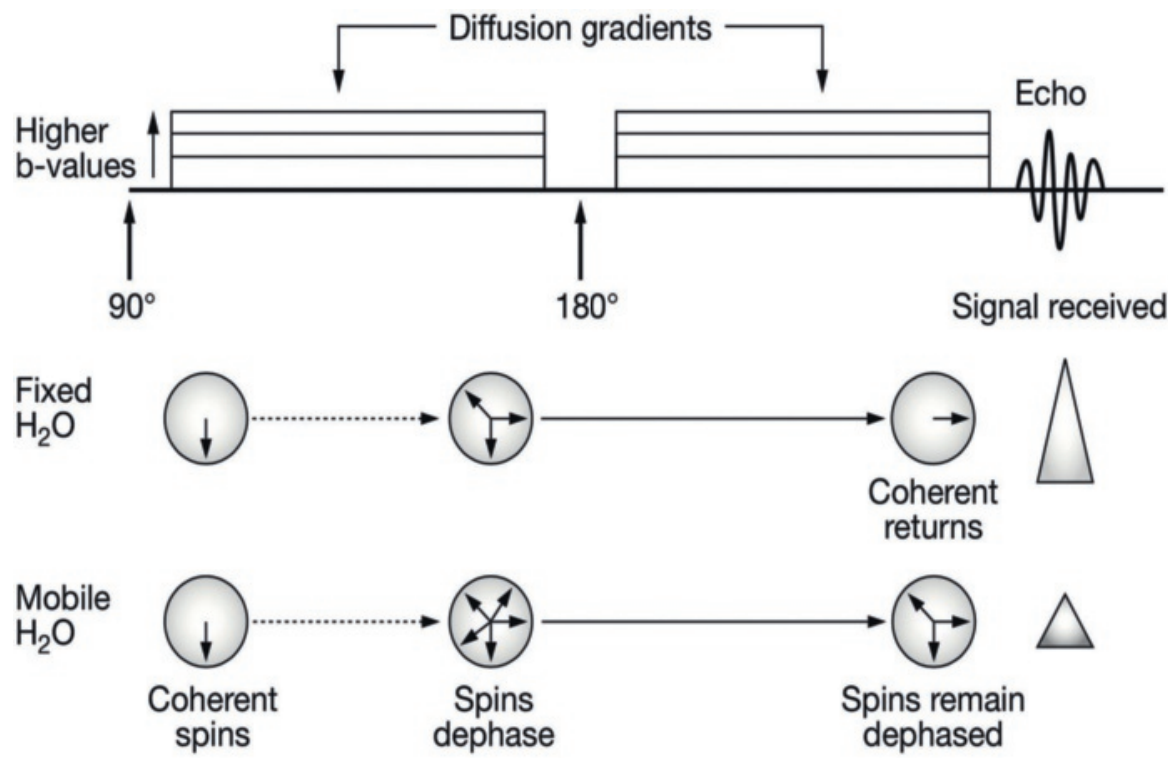

Figure 3.2: schematic 'block scheme' of diffusion MRI signal acquisition. In case of diffusion ('mobile $\mathrm{H}_{2} \mathrm{O}^{\prime}$ ) protons move out of their original location, resulting in a decrease of signal (smaller triangle under 'signal received'). ${ }^{25}$

The method for acquiring diffusion-weighted images with an MRI machine (i.e., the 'acquisition sequence' of applying radiofrequency pulses and switching gradients on and off, see figure 3.2) is designed to gain sensitivity to the water molecules moving ('diffusing') from their original location and is therefore prone to other complications and artefacts than 'conventional' MRI. For example, because the diffusion effect is an effect of signal attenuation, larger voxels or longer scan time is necessary to obtain sufficient signal-to-noise ratio (SNR). To obtain diffusion parameters, the measured signal is fitted to an exponential function, in which the strength of diffusion weighting, expressed as $b$-value, the diffusion-weighted signal and the unweighted signal are used to determine the diffusion coefficient.

During each 'scan', a series of diffusion-weighted images is made for the whole field of view (e.g., thirty diffusion-weighted scan of a kidney). The direction of the magnetic field

25 Reprinted by permission from Macmillan Publishers Ltd. Patterson, D.M. et al. (2008) Technology insight: water diffusion MRI - a potential new biomarker of response to cancer therapy. Nature reviews clinical oncology 5(4) 
gradients is different for each of these images, which allows detecting a difference in diffusion rate in different directions. By fitting the measurements to different models of diffusion, different properties (or 'diffusion parameters') can be obtained. The simplest of these models assumes an exponential relationship between the signal attenuation and the rate of diffusion. By fitting the measurements to this model a parameter that reflects the rate of diffusion (the 'apparent diffusion coefficient,' ADC) can be obtained. An example of a more complex modelling of the diffusion signal is 'diffusion tensor imaging,' (DTI). If the tissue has a clear directional organization (e.g., neural tissue consisting of fibre bundles), the direction of this organization can be determined by making a large number of diffusion images with a great variation of directions of magnetic field gradients. When diffusion is larger in one direction than others, the assumption is that diffusion is less restricted by membranes and macromolecules in that direction, and more restricted in the others, hence that tissue components are mainly structured parallel to that direction. This is called 'anisotropic diffusion'. The measurements obtained by this method are then fitted to a model called the 'diffusion tensor' in order to obtain parameters that provide information on the rate and direction of diffusion.

\subsubsection{The disciplinary perspective}

Using the elements of the matrix, the disciplinary perspective of diffusion MRI can be specified as follows: The goal of diffusion MRI is visualizing microstructural properties of tissues by probing the diffusion NMR signal. The phenomenon that this (sub)field studies is the NMR signal attenuation which is assumed to be the result of water diffusion in tissue. This signal is modelled using several mathematical equations (e.g., the exponential model or the diffusion tensor model) relating the acquired signal to diffusion properties of the tissue. In addition to the theoretical foundations of MRI physics mentioned in the previous paragraph, another fundamental theory to the field is the specific acquisition protocol (the 'acquisition sequence' of applying radiofrequency pulses and switching gradients on and off) that is used to acquire a diffusion signal with MRI. The current objects of investigation and technological advancement in this subfield firstly relate to developing models that more accurately reflect tissue physiology, e.g., with more complex shapes than the diffusion tensor, or multi-exponential fits to model more water motion processes with different velocities. Secondly, diffusion-weighted imaging is applied to different pathologies to investigate the feasibility of detecting changes in tissue structure due to these pathologies. Methods of investigation in this subfield are, similar to larger field of MRI, phantom experiments (to visualize or quantify known and controllable processes in MRI), experiments with healthy volunteers (to investigate the most adequate acquisition and modelling protocol for certain body areas), (clinical) trials with patient populations (to investigate the clinical value of a certain imaging protocol) and computer 
simulations (to simulate the effect of varying parameters). Practical constraints in this disciplinary perspective are similar to the MRI physics perspective, hence formed by the physical MRI apparatus and the computing power of the computer with which data is transformed into images. However, as the diffusion MRI requires the collection of more data, the computing power is of greater importance in diffusion MRI than conventional MRI. The epistemic values in this subfield of MRI are similar to those mentioned in the previous paragraph, although in this particular field, much research is also directed at socalled 'validation', which aims at proving some similarity between diffusion MRI results and anatomy or physiology.

\subsubsection{Signal and image processing}

The signal acquired by MRI machines undergoes many processing steps before they appear as images on the screen. Some of these steps are performed automatically by the MRI system (e.g. deciphering spatial information from the signal to create images) while others require standardized operations in the software package supplied by the manufacturer, and yet other, more advanced, manipulations are performed in custom-made programs or software packages developed for specific research purposes. In the field of diffusion MRI, software packages that perform the most common fitting procedures, usually including recent advances in signal modelling are freely available for the scientific community. However, fitting the signal to uncommon or innovative models requires custom-made algorithms that enable fitting on a voxel-per voxel basis or of the average signal in pre-defined 'regions of interest'. In addition, as the diffusion MRI acquisition protocol is prone to specific artefacts, corrections for these artefacts need to be applied. Many diffusion MRI software packages also facilitate these corrections. However, diffusion MRI is originally developed for brain imaging, and investigating its feasibility in other organs has started more recently and only makes up a small part of the field. New applications are accompanied by additional or new challenges. For example, unlike the brain, kidneys (and other abdominal organs) move up and down as a consequence of breathing. As the calculation of diffusion properties requires combining the signal of multiple diffusion-weighted images at one location, motion artefacts can severely affect the results. This respiratory motion can be prevented during signal acquisition or corrected after. Some methods that should prevent motion artefacts during signal acquisition are not feasible in this case, while others do not completely prevent artefacts as a result of respiratory motion. Therefore, an important aspect of diffusion MRI for the kidneys is motion correction before further signal processing. This requires specific algorithms manipulating the images so that the structures in each separate image are aligned. The principle of alignment of these algorithms is based on the signal intensity of voxels, and the amount of deformation that is allowed in the script. Furthermore, as tissue structure and physiology in the kidneys 
differ from that in the brain, models (as described in 'diffusion MRI') need to be adjusted to that of the kidney. In short, available image processing software packages are usually not suitable to diffusion MRI of the kidneys, hence custom-made scripts are required.

\subsubsection{The disciplinary perspective}

Using the elements of the matrix, the disciplinary perspective of signal and image processing can be specified as follows: The goal of signal and image processing is to turn NMR signals into visualizations and to process images so as to correct for artefacts and to derive parameters from them. The phenomenon of interest in signal and image processing are images as arrays of measurements, each measurement represented by a number or intensity on a grey scale. In that sense, image processing is closely related to signal processing in general. Signals, or arrays of signals, are manipulated by fitting mathematical equations, extrapolation, histogram analysis, noise suppression, etc. Hence, mathematical modelling provides the theoretical basis of this disciplinary perspective. The object of investigation in this field is to improve these manipulations by developing better (e.g., more precise or accurate to the underlying structures) methods for fitting, extrapolation or analysis. Analyses are performed on computers, hence an important aspect of image processing is the available software, which can be quite user-friendly when processing algorithms are standardized for the type of images at hand. For more innovative image applications usually, custom-made algorithms need to be developed, which are programmed in general programming languages such as MATLAB and $\mathrm{C}++$. The central methodology of image processing involves mathematical operations and computer programming, while experts in this discipline think of images as arrays of matrixes of numbers, rather than a representation of shapes and structures inside the body. Simulations are used to study the effect of specific image processing steps. Practical constraints are primarily formed by the computing power of the computer with which data is transformed into images, but also by available software and scripts to realize this transformation. Important epistemic values are the accuracy and precision of the modelling and the validity of the processed signal to the imaged object.

\subsubsection{Medical biology}

In biology, the structure and working of the body is studied at several levels, from the interaction of proteins and other macromolecules within cells to the function of organs. In this research project, the organ of interest is the kidney. Functions of the kidneys are excretion of waste materials, control of blood pressure via hormone excretion, balancing the body fluid, acid-base balance and balancing salts by excretion or resorption of ions. Understanding these functions requires insights into the anatomy, tissue architecture and physiology of the kidneys. The main functional structures of the kidney are 1) the 
nephron, consisting of a tuft of capillaries (the glomerulus) surrounded by membranes shapes like a cup (Bowman's capsule), together responsible for the first filtration of water and small ions, and 2) the renal tubule that is responsible for more specific resorption and excretion of ions and water. The arrangement of small tubes that fan from the centre towards the outside (or cortex) of the kidneys allows maintaining variation in concentrations of ions, which helps to regulate resorption and excretion. See figure 3.3 for an overview of the anatomy of the kidney. Furthermore, each component of kidney tissue contains specific cell types with specific shapes and structures, such as the $\mathrm{Na}+/ \mathrm{K}+$ pump in tubule cells that regulate the concentrations of sodium and potassium in cells and the extracellular matrix, or the shape of 'podocytes' in Bowman's capsule, cells with footshaped processes, or 'pedicles,' that fit together like fingers leaving small slits for filtration. The kidneys also play an important role in blood pressure control, and to that effect are a crucial component of the renin-angiotensin-aldosterone system (RAAS), a hormone system that also involves the liver, several brain regions, the lungs and blood vessels, and regulates plasma sodium concentration and arterial blood pressure. In short, the kidneys are complex organs, consisting of many microscopic structures and fulfilling several crucial functions in the body.

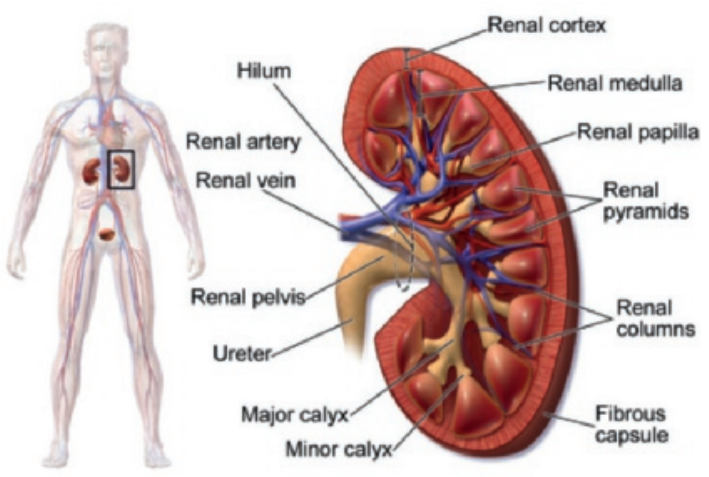

Kidney Anatomy

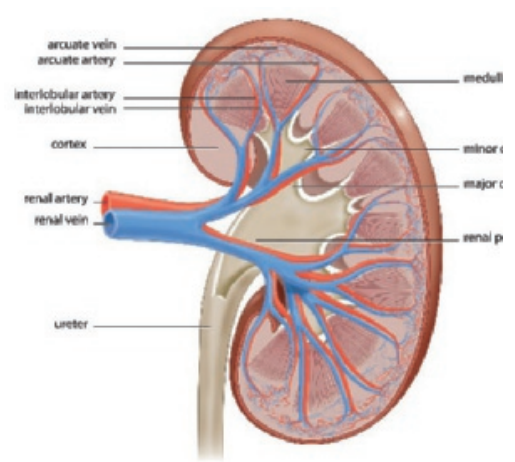

Figure 3.3: anatomy of the kidney ${ }^{26}$

26 From: Blausen.com staff (2014). “Medical gallery of Blausen Medical 2014”. WikiJournal of Medicine 1 (2). DOI:10.15347/wjm/2014.010. ISSN 2002-4436 and http://www.interactive-biology.com/3254/the-anatomyof-the-kidney/, 10-10-2018 


\subsubsection{The disciplinary perspective}

Using the elements of the matrix, the disciplinary perspective of medical biology can be specified as follows: the goal of medical biology is to understand and describe (by visualizations, simple equations or words) mechanisms of human physiology and pathology. The phenomena of interest in the biomedical perspective on kidneys concern the functions and functioning of organs, systems of organs, organ components, cells, cell organelles, etc. (= the phenomena of investigation) and expresses these functions in terms of (micro) architecture, shapes (of organs or cells), molecules (e.g. proteins, hormones and $\mathrm{Na}+$ / $\mathrm{K}+$ pump) and interactions. The object of investigation of the biomedical disciplinary perspective are the mechanisms by which organs etc. fulfil these functions which enable finding causal explanations of phenomena. These mechanisms are usually represented by means of schematic drawings or verbal descriptions. In other words, the way of modelling in this disciplinary perspective is causal-mechanistic, in contrast to imaging disciplines that focus on the morphological/ anatomical structures of organs and mathematical modelling of signals. In physiology, simple formulas are used, for example, to calculate the rate of excretion or resorption of a specific ion. Block schemes are used to express and visualize interactions between organs and the impact of the excretion of a specific hormone on other processes (e.g., inhibitory or stimulatory). Methods of investigation include manipulating cells, proteins and macromolecules in a laboratory environment. Theories and concepts include theories that describe and explain the functions and mechanism of an organ or a system of organs. For example, the mechanism of how the renin-angiotensin-aldosterone system regulates a system of hormone excretion by the kidney, the liver, lungs, the arteries, and the mechanism of how the pituitary gland regulates blood pressure and fluid balance, or the mechanism with which blood is filtered in the glomeruli. Methodology in this disciplinary perspective include methods common in molecular biology, such as studying the cellular architecture of tissues and how molecules (proteins) are produced, excreted, metabolized and taken up by cells. Practical constraints in this disciplinary perspective relate to the availability of instruments to observe or measure features of interest. The mechanisms of interest in this disciplinary perspective are not directly observable: they take place in a living body that cannot be picked apart to look at them. Hence, researchers have to come up with indirect measures and instruments to measure this. Epistemic values are the explanatory and predictive value of a mechanism, consistency with other known mechanisms and functions, and empirical adequacy, in the sense that mechanisms should fit with the clinical observations a clinician makes of his or her patient (from clinical history, physical exam and other observations). 


\subsubsection{Clinical practice concerning patients with kidney tumours}

Finally, an important disciplinary perspective in this research project concerns the patient with a kidney tumour. This perspective differs from the others in that it is not primarily a scientific discipline but a practice with its very own goals, logic, conventions, standardized knowledge and methodologies and epistemic standards (although scientific knowledge and research inform clinical practice). It overlaps with the biomedical perspective on healthy kidneys and on tumour formation. However, the biomedical aspects outlined above are only relevant to the clinical perspective insofar as they have an impact on the clinical aspects of kidney tumours, as signs and symptoms or as possible diagnostic or treatment pathways. Symptoms of kidney cancer include flank pain, haematuria (blood in urine), a palpable mass or general symptoms such as fatigue, loss of appetite or weight, but most kidney tumours are asymptomatic and found incidentally on abdominal ultrasounds, CT or MRI imaging. Several tumour types are distinguished, each with its own histological presentation (visible under the microscope), tumour growth rate and chance of metastases, the most common of which are clear cell renal cell carcinoma (RCC), papillary RCC, and chromophobe RCC. However all tumour types, non-malignant and uncommon tumour types included, appear the same on standard imaging modalities, i.e., as solid lesions. Other diagnostic tests include blood tests for certain ions and proteins and kidney function. When tumours are not metastasized, treatment consists of surgery removing the whole kidney or the part of the kidney that contains the tumour ('radical' or 'partial' nephrectomy). If surgery is not possible, other treatments include chemotherapy or radiation. After surgery, a pathologist examines the tumour tissue to determine the tumour type. Occasionally, the pathologist concludes that the removed tumour was benign.

\subsubsection{The disciplinary perspective}

Using the elements of the matrix, the disciplinary perspective of clinical practice concerning patients with kidney tumours can be specified as follows: the goal of this practice is to diagnose and treat patients with kidney tumours. The phenomenon of interest is 'kidney tumour' as a clinical phenomenon, as something that affects a patient's health and that requires blood tests and imaging to diagnose and come up with a treatment plan that is suitable to the patient. As I have argued in Chapter 1, suitability of treatment is not only dependent on the disease specificities, but also on other features specific for an individual patient, such as age, the presence of comorbidities, a person's general health or the presence of contra-indications to surgery. The objects of investigation that clinicians deal with are kidney tumours, firstly in terms of diagnosis, determining the presence and severity of the disease and next regarding treatment, determining a suitable treatment plan. In the first two chapters, I have laid out a method of modelling that clinician use: fitting togeth- 
er a 'picture' of a patient from heterogeneous sources of knowledge. The methodology of investigation for this disciplinary perspective is determined by clinicians interacting with kidneys in a specific way, i.e., not by directly looking at the organs themselves or the tissue in the microscope (as in the biomedical perspective), but by obtaining (mostly non-invasive) measurements that are often indirect signs of their functioning or integrity. Notably, imaging results appear to clinicians as ready-made pictures on the screen and clinicians focus on the outcome (e.g., shape, size, and presence of the lesions) and what that means to the specific patient, rather than the process with which the images are made (as in the other disciplines). Research in this area is mostly clinical trials, studying the effectiveness end efficacy of treatments to specific patient populations, or the sensitivity and specificity of diagnostic tools. Practical constraints are, firstly, posed by the workings of everyday clinical practice: doctors aim to treat patients with limited means. Both time and resources are scarce and therefore it is impossible to obtain complete information and with that absolute certainty about a diagnosis. In addition, treatment options are usually limited and each treatment is associated with side effects. Doctors deal with patients that have their own values, desires and expectation, which also limits the possible treatment options. Clinical trials deal with another range of practical constraints: for example, ethical constraints when dealing with human subjects and organisational constraints when dealing with large numbers of subjects. Epistemic values for this disciplinary perspective are relevance and reliability of information for the patient population and individual patients. Theories and concepts in this disciplinary perspective include theories about the nature of disease and treatment, such as the naturalist conception of disease, saying that the human body contains organs and organ systems with functions that can be disturbed by different causes ways, resulting in 'disease' (Boorse, 1975). 
Table 1

\section{Disciplinary Goal perspective}

\section{Phenomena of} interest

\section{Object of investigation}

\section{Modelling}

Magnetic fields, proton spins, radiofrequency pulses, relaxation times

Developing
hardware,
developing
acquisition
schemes, software
development

Mathematical modelling of diffusion signal Block schemes visualizing acquisition schemes

$\begin{array}{lllll}\text { Diffusion } & \text { Visualising } & \text { NMR signal } & \text { Modelling the } & \text { Mathematical } \\ \text { MRI } & \text { microstructural } & \text { attenuation due to } & \begin{array}{l}\text { diffusion signal, } \\ \text { modelling of }\end{array} \\ & \text { properties of tissues } & \text { proton diffusion } & \text { investigating the } & \text { the diffusion } \\ \text { by probing the } & \text { in tissue } & \begin{array}{l}\text { diffusion signal } \\ \text { diffusion NMR signal }\end{array} & & \text { in body tissues }\end{array}$

\begin{tabular}{|c|c|c|c|c|}
\hline $\begin{array}{l}\text { Signal and } \\
\text { image } \\
\text { processing }\end{array}$ & $\begin{array}{l}\text { Turning signals } \\
\text { into visualizations, } \\
\text { correcting artefacts, } \\
\text { etc. }\end{array}$ & $\begin{array}{l}\text { Images as arrays } \\
\text { of measurements }\end{array}$ & $\begin{array}{l}\text { Developing } \\
\text { better methods } \\
\text { for fitting, } \\
\text { extrapolating or } \\
\text { analysis of the } \\
\text { diffusion signal. }\end{array}$ & $\begin{array}{l}\text { Mathematical } \\
\text { modelling of } \\
\text { the diffusion } \\
\text { signal }\end{array}$ \\
\hline $\begin{array}{l}\text { Medical } \\
\text { biology }\end{array}$ & $\begin{array}{l}\text { Understanding } \\
\text { and describing } \\
\text { mechanisms of } \\
\text { human physiology } \\
\text { and pathology }\end{array}$ & $\begin{array}{l}\text { The function of } \\
\text { the the human } \\
\text { body at several } \\
\text { levels }\end{array}$ & $\begin{array}{l}\text { Mechanisms of } \\
\text { bodily functions }\end{array}$ & $\begin{array}{l}\text { Causal- } \\
\text { mechanistic }\end{array}$ \\
\hline $\begin{array}{l}\text { Patient with } \\
\text { a kidney } \\
\text { tumour }\end{array}$ & $\begin{array}{l}\text { Diagnosis and } \\
\text { treatment of patients } \\
\text { with kidney tumour. }\end{array}$ & $\begin{array}{l}\text { Kidney tumours } \\
\text { as a clinical } \\
\text { phenomenon }\end{array}$ & $\begin{array}{l}\text { Kidney tumours } \\
\text { in terms of } \\
\text { diagnosis and } \\
\text { treatment }\end{array}$ & $\begin{array}{l}\text { Fitting together } \\
\text { a 'picture' of a } \\
\text { patient }\end{array}$ \\
\hline
\end{tabular}




\begin{tabular}{llll}
\hline $\begin{array}{l}\text { Theories and } \\
\text { concepts }\end{array}$ & $\begin{array}{l}\text { Methodology/ } \\
\text { instruments }\end{array}$ & Practical constraints & $\begin{array}{l}\text { Epistemic values/ } \\
\text { scientific ideals }\end{array}$
\end{tabular}

\begin{tabular}{llll}
\hline $\begin{array}{l}\text { Classical } \\
\text { electrodynamics, } \\
\text { nuclear magnetic }\end{array}$ & $\begin{array}{l}\text { Phantom } \\
\text { experiments, clinical } \\
\text { trials with patients }\end{array}$ & $\begin{array}{l}\text { Physical MRI } \\
\text { apparatus, computing } \\
\text { power }\end{array}$ & $\begin{array}{l}\text { Consistency, reliability, } \\
\text { validity }\end{array}$ \\
transforms & $\begin{array}{l}\text { and/or healthy } \\
\text { volunteers }\end{array}$ & &
\end{tabular}

$\begin{array}{llll}\text { See 'MRI physics' } & \text { See 'MRI physics' } & \begin{array}{l}\text { Physical MRI } \\ \text { apparatus, computing } \\ \text { Diffusion MRI }\end{array} & \begin{array}{l}\text { Consistency, reliability, } \\ \text { validity }\end{array} \\ \text { acquisition } & & \text { power }\end{array}$

\begin{tabular}{|c|c|c|c|}
\hline $\begin{array}{l}\text { See 'MRI physics' } \\
\text { Matrix mathematics, } \\
\text { data science }\end{array}$ & $\begin{array}{l}\text { Mathematical } \\
\text { operations } \\
\text { and computer } \\
\text { programming }\end{array}$ & $\begin{array}{l}\text { Computing power and } \\
\text { available software to } \\
\text { transform data into } \\
\text { images. }\end{array}$ & $\begin{array}{l}\text { Accuracy and } \\
\text { preciseness of models } \\
\text { validity }\end{array}$ \\
\hline
\end{tabular}

$\begin{array}{llll}\begin{array}{l}\text { Theories describing } \\ \text { and explaining } \\ \text { (kidney) function }\end{array} & \begin{array}{l}\text { Molecular biology, } \\ \text { (i.e. looking at the } \\ \text { tissue through a } \\ \text { microscope) }\end{array} & \begin{array}{l}\text { Available measures and } \\ \text { instruments. }\end{array} & \begin{array}{l}\text { Explanatory and } \\ \text { predictive value of a } \\ \text { mechanism, empirical } \\ \text { adequacy, }\end{array}\end{array}$

$\begin{array}{lll}\begin{array}{l}\text { Theories about the } \\ \text { nature of disease and } \\ \text { treatment }\end{array} & \begin{array}{l}\text { Clinical history, } \\ \text { imaging, lab results, } \\ \text { pathology reports. } \\ \text { Clinical trials }\end{array} & \begin{array}{l}\text { Limitation of resources. Relevance, reliability, } \\ \text { Patient values, Ethical } \\ \text { and organisational } \\ \text { constraints for clinical } \\ \text { trials. }\end{array}\end{array}$




\subsection{Combining disciplinary perspectives}

To develop a new imaging tool for clinical practice that uses diffusion-derived parameters to characterize kidney tumours, each of the above perspectives needs to be effectively combined in regard to the target system. Each discipline has its own goal(s) and phenomena of interest, which are elements of the disciplinary perspective that an expert has. Each discipline is crucial for a successful solution because experts from different discipline have different views (i.e., disciplinary perspectives) on the target system (which consist of the MRI machine, the body containing kidney and kidney tumour, software operating the MRI system and processing the images, and the clinical practice in which the images will play a role). The disciplinary perspective of each disciplinary expert enables each of them to focus on and perform epistemic activities concerning the target system, each of them thus making different contributions. The issue is that, in order to achieve a common goal (developing an imaging tool that improves diagnosis and treatment of individual patients), disciplinary experts need to combine their expert views. Considering the differences between the relevant disciplinary perspectives sketched above already makes clear why this is a challenge.

This interdisciplinary endeavour - to develop an imaging tool that makes use of diffusion MRI to distinguish between different kidney tumour types in order to prevent unnecessary surgery - does not necessarily require integration of theories from different fields in order to produce a new, interdisciplinary understanding of the target system. Rather, experts having different disciplines are organized around the target system and interact in the process of studying and further developing knowledge, hypothesis, critical questions, insights and ideas regarding aspects of the target system. Experts usually approach the target system from their own disciplinary perspective and from that perspective come up with solutions appropriate to that discipline, for problems raised within that discipline. Conversely, an interdisciplinary product is achieved by interdisciplinary collaborative reasoning, where each expert, from their own disciplinary perspective, contributes in reasoning about phenomena indicated by other disciplinary perspectives. For example, the perspective of MRI physics allows for reasoning about how protons in tissue respond to magnetism and how this results in signals and contrast in images. However, applying MRI to specific body parts, also requires reasoning about the composition of tissues, for instance, high in water or fat, very solid or air-filled, the exact location and other structures in the proximity of the organ of interest, and whether the body part moves (e.g., respiratory motion, blood flow, heart beat or peristalsis of the intestines), in order to determine the most suitable acquisition protocol. Recognizing and including these additional aspects is facilitated by the biomedical disciplinary perspective. In kidney imaging, knowledge of 
the location and size of the kidneys (which belongs to biomedical expertise) determines the choice of MRI coil (which belongs to MRI physics expertise). Additionally, knowing that the intestines (containing gas and making specific movements as they digest food) are closely located to the kidney (which again belongs to biomedical expertise) introduces a challenge as this might cause artefacts (like gas-tissue transition artefacts and motion artefact, which belongs to MRI physics expertise) as does the movement of kidneys as a consequence of breathing. To predict or understand the specificities and complexities of kidney imaging with MRI thus requires experts from different disciplinary perspectives to interact, by moving between the perspective of MRI physics to understand how the desired signal contrast and undesired artefacts are produced, and the perspective of biomedicine to determine which of these issues are at play in kidney imaging, and again back to the perspective of MRI physics to reason about possible ways to prevent or correct for the undesired artefacts (see figure 3.4).
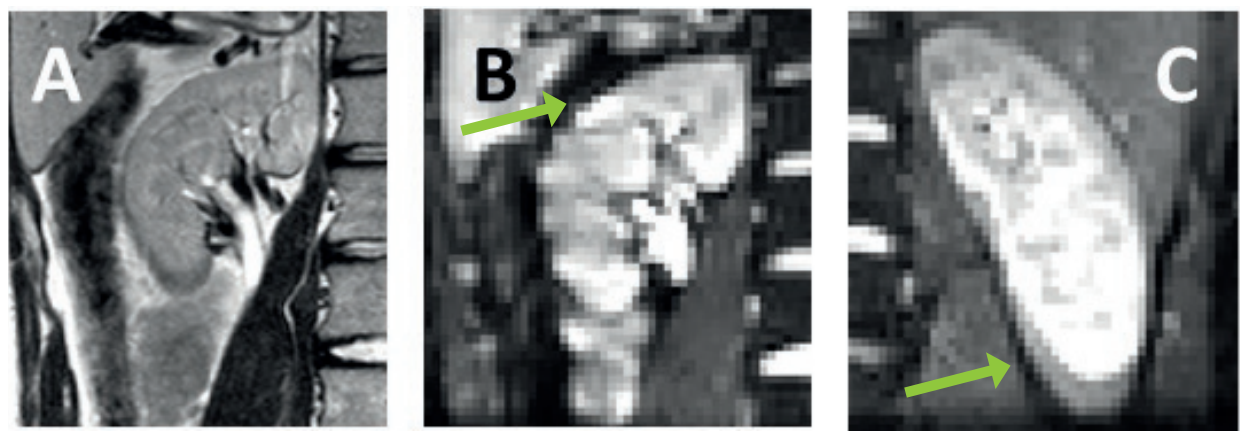

Figure 3.4: examples of common artifacts in diffusion-weighted imaging of the kidneys

(A) An anatomical (T2) scan of the kidney. (B) the transition between tissue types results in magnetic field inhomogeneities, which shows as so-called 'susceptibility' artifact at the edge of the kidney. (C) respiratory motion in the longitudinal direction results in blurring of the kidney underpole (arrow).

More specifically, in comparison with 'conventional' MRI (i.e., in the established clinical uses), other aspects become relevant in research that aims to develop diffusion MR imaging of kidney tumours. Firstly, because a specific 'acquisition sequence' of applying radiofrequency pulses and switching gradients on and off is used for the acquisition of the diffusion MR signal, this type of MRI is (more) prone to specific artefacts, for instance, artefacts caused by transitions between different kinds of materials such as tissue and air, the so-called 'susceptibility artefacts.' Moreover, because the image contrast is based on a signal attenuation, a larger voxel size is needed to obtain an adequate signal to noise ratio (SNR). Understanding and dealing with these specific aspects involves interdisciplinary collaborative reasoning about diffusion MRI signal acquisition from the perspective of MRI physics and taking into account the perspective of MRI diffusion - as this sub-dis- 
cipline of MRI considers the specificities related to the development of diffusion-weighted imaging technology. Secondly, the signal contrast in diffusion MRI differs from that of conventional MRI and is based on the principle of Brownian motion of water molecule in tissues, affected by microstructural properties of tissue, such as the density of macromolecules and membranes, rather than the behaviour (spins and relaxation - see Paragraph 'MRI physics') of water molecules interacting with other molecules within a certain tissue type. Again, hypothesizing about the expected outcome of diffusion parameters in the kidneys based on the signal contrast requires collaborative interdisciplinary reasoning of experts with different disciplinary perspectives. Experts with a biomedical perspective will focus their reasoning on the tissue architecture. They imagine, for instance, the structure and materials of the pyelum, which is the central part of the kidney where urine is collected and basically consists of a fluid-filled cavity, whereas the kidney cortex (the outermost area of the kidney) is highly vascularized, and the medulla (the middle part) consists of radially organized tubules. The biomedical expert may also include (possible) water motion processes in the kidney, such as the flow of pre-urine in the tubules, unrestricted free water in the pyelum, and blood flow in the large vessels in the kidney. Based on this picture, the biomedical expert can make a connection with the MRI and DTI experts by pointing out how diffusion (or movement) of water molecules will be affected in different types of kidney tissue. In this manner, the biomedical expert has made the diffusion processes in the kidney accessible to the diffusion MRI expert. The mechanism sketched by the biomedical expert can then be viewed from the perspective of diffusion MRI to hypothesize which aspects can be picked up by diffusion MRI and how they might be represented by parameters obtained by different diffusion MRI models. For example, as described in Paragraph 3.2.2., the DTI (diffusion tensor imaging) model allows obtaining parameters that represent the direction in which the highest rate of diffusion is detected (and thus water motion is least restricted). This might provide interesting outcomes in the medulla, as the medullar tissue is organised in tubules that radiate from the centre to the kidney cortex along which water molecules can easily diffuse (which can be detected using DTI, thus making visible the structure of the medulla, see figure 3.5). The parameters thus obtained can be mapped into an image of the kidney, and MRI experts looking at these images from a diffusion MRI perspective are able to reason about what the differences in parameters obtained for specific regions can tell about the structure of the underlying tissue (e.g., a high diffusion rate in one direction and not others means a high structural organisation). Subsequently, an expert with a biomedical perspective can reason about what this image tells about the integrity and function of the underlying tissue (e.g., when diffusion is similar in all directions in an area where this is not expected, it might mean that tubules 'leak' and thus do not function well). In addition, the expert with an image processing perspective develops scripts to translate the signal into specific numerical values, 

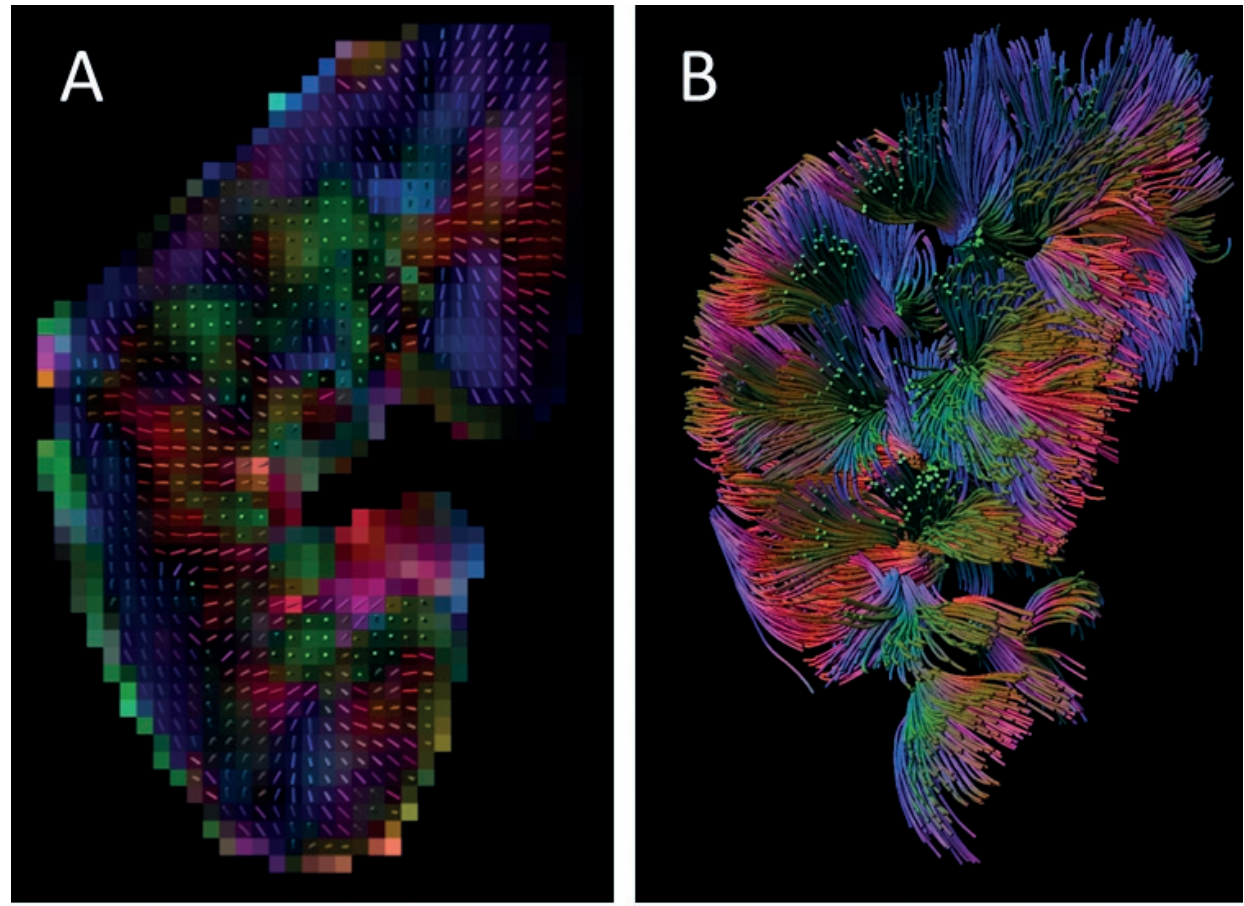

Figure 3.5: Diffusion tensor imaging and tractography in the kidney

(A) Lines show the preferred diffusion direction at different areas of the kidney. (B) these lines can be 'tracked' to visualize organised structures.

either for each voxel in the kidney volume to form parameter maps or for a 'region of interest' (e.g., the kidney tumour) to obtain a sort of 'measurement.' These parameters are obtained by fitting the signal to a mathematical equation that expresses the relations between the signal and a diffusion parameter. Concerning this process of fitting the signal to these mathematical equations, two aspects can be considered by experts involved in this process. First, the accuracy of the model to what it is supposed to be modelling, namely diffusion of water in renal tissue by the biomedical expert. Second the correctness of the fit between the model and the signal by the MRI expert. These two aspects (accuracy and correctness) bring forth different criteria of signal fitting with which the image processing expert has to deal. A model fit can be more or less accurate, which refers to how well it resembles the measured underlying tissue properties. On the other hand, a fit can be more or less precise, meaning that it follows all details of the measured signal (including noise and artefacts) more or less closely. These criteria require different evaluative approaches. Preciseness is usually evaluated in terms of mathematics through algorithmic evaluation (e.g. goodness of fit, a statistical evaluation of a model fit), which is preferred by the more technological disciplinary perspectives (i.e., MRI physics, diffusion MRI and signal and 
image processing) whereas accuracy requires some type of comparison with a (validated) parameter that represents the same or a closely related physiological property, which is preferred by the medical perspective (i.e., biomedical and clinical practice concerning patients with kidney tumours). In summary, to understand what information parameters obtained from diffusion MRI can provide about the kidneys, switching between the MRI physics, diffusion MRI, image processing and the biomedical perspective is required.

My analysis above aims to show that the epistemology of interdisciplinary collaboration between disciplinary experts - who, in this case, aim to develop an imaging technology for the diagnosis of kidney tumours - is better understood by taking into account (at a more abstract level) the disciplinary perspectives that each expert exercises, then in terms of 'representational knowledge' contributed from each discipline that must somehow be integrated. An advantage of analysing the collaborative interdisciplinary reasoning process in terms of disciplinary perspectives is, for instance, that a clinician -who takes the effort to understand the epistemological difficulties of a collaboration in terms of distinct disciplinary perspectives that can be explicated - may start to see that the image cannot be understood as a 'photograph', i.e., that there is not a straightforward relationship between the acquired signal and the images. Instead, by explicating the specific signal and image processing perspective of the MRI expert, the clinician starts to see that the signal is first fitted to a model before deriving parameters. Looking at this interdisciplinary teamwork in detail, we see that these experts move from one disciplinary perspective to another when discussing aspects of the target system. I will argue that recognition of the disciplinary perspectives as a cognitive framework within which each of them reasons and approaches the target system, helps to better understand each other. For example, as I have illustrated above, the expert with a biomedical perspective contributes with knowledge about the structure and functions of kidney including understanding of mechanisms that determine the (dis)function of kidney, the expert with a diffusion MRI perspective contributes by reasoning about the signal picked up by the machine, and the image processing expert contributes by designing or developing a way to translate the signal into specific numerical values.

\subsection{Interdisciplinary collaboration in developing medical tech- nology for clinical practice}

As has been emphasized, interdisciplinary collaboration in developing medical technology for clinical practice does not primarily aim at the integration of theories or concepts, but at an understanding of the target system that helps in developing the technology. The goal 
of this research project was to develop an imaging tool that makes use of diffusion MRI to distinguish between different kidney tumour types in order to prevent unnecessary surgery. The question that must be asked by the research team is whether the technology contributes to the intended clinical practice. This means that the parameters and images that are developed should provide information that is relevant to the clinical practice concerning patients with kidney tumours. For example, the information obtained by the imaging tool cannot be acquired by other, already existing procedures and contributes to clinically better decisions regarding treatment (e.g., a surgery can be prevented) in regard of a significant patient population. An additional requirement is that the new imaging technology is understandable and interpretable by clinicians. Meeting these requirements means that eventually, all experts engage in a clinical perspective. The clinical perspective involves practical issues such as defining the patient population, the patients' needs, what information is already available about these patients in standard clinical practice, the level of uncertainty in old and new diagnostic techniques, what treatment options are available, and how decisions between these options are made. Identifying these issues by exercising a clinical perspective provides input for further consideration exercising the other disciplinary perspectives. For instance, a biomedical perspective allows determining which physiological mechanisms that can possibly be characterised by this new imaging technology are clinically relevant to the patient population. The biomedical perspective states, for example, that tumours are usually highly vascularized and have a high cell density, and will, therefore, show differently on diffusion MRI from healthy tissue, which would make this an attractive technology to be developed. However, from a clinical perspective, it will be argued that most tumours can also be visualized with 'conventional' imaging modalities, and therefore the MRI-DTI does not simply amount to clinically relevant mechanisms or measurements. Hence, further directions for development of this technology need to be considered, exercising an MRI diffusion perspective, to find parameters that can provide new information about the tumour tissue and allows making distinctions between tumour types.

For a clinically relevant imaging modality, the challenge is thus to find a physiological mechanism that (a) distinguishes, in this case, between benign and malignant kidney tissue, (b) can be picked up by the diffusion signal and visualized or represented in diffusion parameters, and (c) that also warrants improved diagnoses and treatment. Again, assessing this requires contributions from all disciplinary perspectives.-For example, only by a collaboration between experts with a biomedical and a diffusion MRI perspective, respectively, can it be found that a certain parameter (e.g., fractional anisotropy, FA) is specifically indicative of a certain kidney cancer type. This conclusion has to be in agreement with both disciplinary perspectives, i.e., the known physiology of that tumour should 
support the idea that a specific structural arrangement of cells can be found, and the mathematical model applied to fit the signal and obtain parameters should either be one that is commonly accepted in the field of diffusion MRI or developed according to methodology accepted in that disciplinary perspective. The same goes for the signal and image processing protocol, developed by an expert from the field of image processing. Developing a new application requires innovative protocols to process the diffusion signal so that the preferred parameters can be obtained. However, the image processing field from which the existing protocols stem have established internal validity. Therefore, innovating these protocols should not only be aimed at being more adequate to the intended application, but also at maintaining scientific rigour by following established methodologies accepted in the disciplinary perspective of image processing.

\subsection{Disciplinary and interdisciplinary experts: The role of tech- nical medicine}

The 'disciplinary perspectives' that I describe above are embodied by different experts. In the research project that I describe here, several disciplinary experts were involved: the medical perspectives are embodied by a radiologists who is the expert on the interpretation of clinical images and the imaging options that are currently available to visualize properties of kidney tumours (and what properties they can visualize), and by a urologists who diagnoses and treats patients with kidney tumour, whereas the technical perspectives are embodied by a biomedical engineer who designs acquisition protocols and develops image processing scripts, and a computer scientist who developed a software program specifically for processing and visualization of diffusion-weighted MRI data. Although I have defended the importance of collaborative interdisciplinary reasoning, in the actual case, these experts did not directly interact much with each other. In fact, the professionals are located at a different institution in a different city and some of them have never met each other.

Instead of experts directly interacting with each other, my own role as a professional in technical medicine has been to take the role of 'connector', communicating with members of the research team, most often individually. In Paragraphs 3.7 and 3.8 of this chapter I will reflect on this specific role that technical physician can fulfil in interdisciplinary collaborations in more detail. Being the main investigator, I was in charge of collecting, processing and analysing data, in collaboration with the other experts. Communication often focused on practical things: which patients to include and when to plan the MRI scan. From my background in technical medicine, in which I have learned to systematically 
apply knowledge from several disciplines, including medical sciences, natural sciences and technology, I consider myself an 'interdisciplinary expert'. Below I will explicate what I mean by 'interdisciplinary expertise,' but first I will give an illustration of it by describing my (epistemic) activities as an interdisciplinary expert in an interdisciplinary research project. My role in this research project was to synthesize contributions from the disciplinary experts into a product that was both technologically sound (i.e., consistent with MRI physics, precise modelling of the signal, reliable acquisition and processing) as well as clinically relevant (i.e., using the tool in a reliable and valid way to obtain information about the patient's condition, that cannot be obtained in any other way). Therefore, communication also took place at a higher level: I was trying to uncover those features of the target system under development that would make it a success from the viewpoint of each disciplinary perspective.

For example, I talked with the urologists to figure out what information he would actually need to improve the treatment of patients with kidney tumours, which led us to ideas about what type of discriminations the technology should be able to make - that is, between different kidney tumour types, in other words, between kidney lesions consisting of a specific tissue and cell type. To formulate a research question in terms of diffusion MRI, this clinical question (what kidney tumour type does my patient have?) has to be translated into a question that can be answered using diffusion MRI. This means knowing what kind of differences in tissue microstructure lead to differences in the diffusion MRI signal (e.g., those that restrict water motion) but also the specific properties of kidney tumour types that might lead to those differences (e.g., a higher cell density). This allows making a prediction of the expected diffusion signal in a certain tumour type. Thus, in addition to a clinical goal (to distinguish between kidney tumour types), my collaboration with the urologist had a technological goal: to develop the image acquisition and processing protocol to establish those diffusion parameters that are expected to differ between kidney tumour types. In other words, for this research project to be successful, I had to keep in mind two goals, a clinical one and a technological one, that would be evaluated from different disciplinary perspectives and that involve different epistemic values. A good interdisciplinary research outcome does not only have (integrated) contributions from multiple disciplinary perspectives but is also consistent with those perspectives in terms of content as well as methodology and epistemic values. Therefore, the challenge for interdisciplinary experts is to identify where this may cause conflicts (e.g., while the diffusion MRI disciplinary perspective values preciseness and accuracy of models and therefore focuses on correctly fitting the signal, the clinical perspective values the explanatory and predictive power of a measurement and therefore focuses on how the parameters obtained from fitting the signal relate to the patient's condition) and find ways to deal with this. 
To effectively combine the disciplinary perspectives of the urologist and MRI experts, I (as a professional in technical medicine) thus needed to be able to bridge between them. I aim to analyse the so-called interdisciplinary expertise of a professional in technical medicine in terms of two cognitive abilities, in which the notion of disciplinary perspectives introduced in this chapter plays a key role. Firstly, the technical medicine professional needs to recognize and get acquainted with each of the disciplinary perspectives on the target system of the experts (especially: goal, phenomena of interest, object of investigation, modelling, theories and concepts, methodology/ instruments, see Table 1). This does not mean that these professionals are experts in each of these disciplines (in that case, the involvement of disciplinary experts would not be necessary). Rather, they need to understand the disciplinary perspectives at a more abstracts level, to such an extent that they are able to effectively communicate and (partly) understand the views and approaches to the target system that results from that disciplinary perspective. Secondly, the technical medicine professional needs to connect the specific epistemic contribution of each expert. This requires reflecting on the different disciplinary perspectives as well, focusing on their practical constraints, epistemic values and scientific ideals (Table 1), in order to identify and formulate the medical and technological goals, the requirements that follow from these goals, how the results would be evaluated from their respective perspectives, and whether this would lead to conflicts in the design, execution and reporting of the study. The cognitive and epistemic tasks of communicating and connecting in an interdisciplinary research project require higher-order cognitive skills at different levels. 'Level 1 higher-order cognitive skills' allow performing epistemic activities from within disciplinary perspectives (in collaboration with disciplinary experts), as well as the switching between them. 'Level 2 higher-order cognitive skills' are a specific type of meta-cognitive skills, and concern the ability to reflect on the disciplinary perspectives themselves.

\subsection{Understanding interdisciplinarity}

My account of disciplinary perspectives and how they play a role in research aimed at a target system goes against usual conceptions of what interdisciplinarity involves. Interdisciplinarity is often defined in contrast to multidisciplinarity. In this type of definition, the characteristic difference between multi- and interdisciplinarity is that the latter achieves a certain integration or synthesis of theoretical or methodological concepts, whereas in multidisciplinary work theoretical concepts and methodological approach is kept intact (Huutoniemi et al. 2010; Klein 2010). However, most definitions of interdisciplinarity lack a clear idea of what integration means. Often, metaphors are used that are not wrong, but too limited. One metaphor about the integration of knowledge is that bits of knowledge 
are fitted together as in a jig-saw puzzle. Another is that someone's knowledge about a problem has certain gaps that can be filled by retrieving information from other disciplines. These metaphors about integration of knowledge are built on a positivistic or a scientific realist notion of knowledge, respectively. These two ideas about knowledge both heavily rely on the so-called empiricist view that observations and measurements are the ultimate sources of knowledge. At the same time, this implies that (scientific) knowledge represents aspects of observable (says the positivist) and unobservable (says the scientific realist) aspects of the world in a more or less literal way. In other words, these notions of knowledge (erroneously) suggest that knowledge simply and objectively represents aspects of the world, which implies that the world speaks for itself, and conversely, that (scientific) knowledge speaks for itself, and therefore, that combining knowledge from different disciplines is unproblematic i.e., the jigsaw-puzzle and information-gap metaphor suffice to understand knowledge integration. In contrast, I have argued that the world and scientific knowledge do not speak for themselves, but are shaped by a set of aspects grasped by the disciplinary matrix (and summarized in Table 1) like goals, phenomena of interest, object of investigation, ways of modelling, theories and concepts, methodology and instruments, and epistemic values and scientific ideals, together forming a disciplinary perspective.

Additionally, my conception of interdisciplinarity focuses on another type of integration than most authors in the philosophy of science seem to deal with, i.e., the integration of theory or concepts. Instead, in the interdisciplinary research in my case, aspects of the disciplinary perspectives are not integrated. Rather, what makes this research project interdisciplinary is that each disciplinary perspective contributes to the solution by coherently establishing links between ${ }^{27}$ perspectives. As I have illustrated, reasoning processes are

27 The notion of interdisciplinary as 'linking' disciplines or certain aspects of disciplines has been put forward earlier by Nancy Nersessian and Christopher Patton (2009), who have studied several biomedical engineering labs and argue that mental, physical and computer models function as "hubs for interlocking biological and engineering concepts, methods, and materials, mental and external representations in model based inference, design and history and research and learning." (Nersessian and Patton 2009, pg. 3) They call this 'interlocking models.' It is through these interlocking models that disciplines are combined into (epistemic) objects that both engineers and biologists can reason with. Hanne Andersen (2016) argues that in interdisciplinary research, team members with different disciplinary backgrounds interlock cognitive resources. With this notion, she aims to focus on "the process of interlocking partial contributions from different areas of expertise in the production of genuine research contribution in a cognitively divergent research field" (Andersen 2016, footnote 5). This requires of these disciplinary specialist to develop additional skills to their contributory expertise, which Anderson calls 'interlocking expertise.' 
crucial to how the linking of disciplines actually takes place. The outcome of reasoning from one perspective provides input - questions, problems, answers, hypotheses, and knowledge shaped by the disciplinary perspective - in another perspective in a way that eventually results in a problem solution that is internally coherent as well as consistent (i.e., that is not in conflict with commonly accepted theories, correct usage of instruments and epistemic standards) with the contributing disciplines. Rather than integration of disciplines, I propose the notion of disciplinary perspectives interacting around a problem concerning a specific target system.

Schmidt (2011) argues that there are multiple types of interdisciplinarity (ID), classified according to well-established distinctions in the philosophy of science; object-oriented ID ('ontology'); ${ }^{28}$ theory-oriented ID ('epistemology'); ${ }^{29}$ and method-oriented ID ('methodology'). ${ }^{30}$ Schmidt advocates a fourth type of interdisciplinarity, which according to him is the most needed as well as the most challenging type: problem-oriented interdisciplinarity. According to Schmidt, problems consist of three elements: an undesired (initial) state, a desired (goal) state, and the barriers to get from one to the other. Problems are external to disciplines and, in Schmidts conception, primarily societal. Schmidt argues that problem-oriented ID cannot be regarded as value-free research: "the notion of problem can be regarded as a reflexive term that calls for an explication of who is considering what as a problem and why," (Schmidt 2011, p. 258). Because the type of problems that Schmidt has in mind do not have clear solutions the way puzzles do, Schmidt excludes engineering sciences, as they are "able to come up with a new artefact to solve a technical problem,” (Schmidt 2011, p. 259). Therefore, at first sight, my case does not seem to fit well with Schmidt's conception of problem-oriented interdisciplinarity. However, I think that this overlooks a type of problem that engineering or engineering sciences often deal with: real-world problems that require (or might be solved by) technological solutions. In those

28 According to Schmidt, object-oriented ID is predominant in engineering sciences, where the focus is on the development of technologies and analyzing their consequences, whereas problem-oriented ID is much wider and comprehensive and not just about technological problems. According to Schmidt, theories and concepts may serve as means but are not ends of problem-oriented ID.

30 Developing methods is not a goal of problem-oriented ID. Rather, it is about selecting the right method to solve the problem; "Anything goes - if it serves for the solving of problems," (Schmidt 2011, p. 269). Here, I tend to disagree. If disciplinary perspectives are involved in solving the problem, then the most appropriate methods, or instruments from these disciplines must be selected, and used in accordance with the standards from that disciplines. 
cases, the problem is also loaded with value and calls for an explication of who is considering what as a problem and why, to define a workable direction for solving the problem. Additionally, a designed or developed artefact has to function in a complex real-world system and its exact use (by whom doing what) cannot be predicted, but nevertheless has to be taken into consideration while working on it. Hence, to present these as simple puzzles that are solved by coming up with a technological artefact passes over the complexities that make these types of problem interesting examples of his own framework.

Applying Schmidt's framework to my case reveals interesting aspects of the case and the type of interdisciplinarity that I am trying to construe. Unlike Schmidt's framework, I did not deal with a societal but a medical problem, but in my view the problem (the characterization of kidney tumours by a new type of MRI) is nevertheless a real-world problem in the sense that it is a problem external to specific disciplines: it is not necessarily a technical problem or a problem of our understanding of tumour physiology, and the solution will have to be taken up in a complex context involving many stakeholders with different tasks, expertises, purposes and needs as well as an institutional and technological system. The three elements that Schmidt mentions are fruitful to structure the understanding of the problem and goal of the project, and how the disciplines are involved in problem definition. The undesired (initial) state is the situation where patients receive unnecessary surgery because it is impossible to determine whether a patient suffers from a benign or malignant kidney tumour, the desired (goal) state is a situation in which doctors can non-invasively detect what type of kidney tumour patients have. Barriers include the lack of a method for detecting the kidney tumour type, which has to be developed, but later on also the acceptance and integration of the methods into the clinical system, as well as more practical barriers such as financial and institutional barriers. According to Schmidt, a large proportion of the epistemic work in interdisciplinary is setting the problem from the angle of the target solution: i.e., a problem is defined when a more desired state can be imagined. This requires involvement from multiple disciplines: to see the initial situation as undesired requires a clinical perspective, but to see it as a problem for which a solution can be developed requires imagining a goal state, or in other words the involvement of a discipline that offers a possible solution. In practice, the problem definition and set-up of the target system only emerged when I, in my role of technical medicine professional, started talking to clinicians and combining this with what diffusion MRI had to offer regarding their clinical questions.

Thorén and Persson (2013) also focus on real-world problems rather than the integration of theories and concepts. They present cases of interdisciplinary research in sustainability science to argue that traditional accounts of interdisciplinary research (focusing on 
the integration of theories and concepts) are inappropriate for that field and show that interdisciplinary solutions are often obtained without the integration of theories and concepts. Instead, Thorén and Persson introduce the notion of problem-feeding in which problems are moved from one discipline to another: "new social problems emerge as a result of changes in the natural world," (Thorén and Persson 2013, p. 346). In my example, I do not go as far as to claim that a medical problem emerges as a technical problem, but similar to Thorén and Persson I show that one of the first steps in coming up with an interdisciplinary solution is translating the medical goal (how can I distinguish between different kidney tumour types) into a technological goal, which in turn leads to all kinds of technological problems (e.g., how to correct for artefacts due to motion, and how to model the diffusion MRI signal). Thorén and Persson argue that there are two types of problem-feeding: unilateral and bilateral. In unilateral problem-feeding, one discipline is the source of problems for another discipline. According to Thorén and Persson, this is the type of problem-feeding that is best fitting to sustainability science. In my case, the empirical aim is not explanatory, as in sustainability science, but to intervene, to improve clinical practice hence to develop or design a technology. Therefore, supposedly, the (technological) solution feeds back into the medical discipline, which is a case of bilateral problem-feeding in Thorén and Persson's framework. According to them, for the solution to feed back into the problem-setting discipline, it should be admissible. Auxiliary (but not necessary or sufficient) for this type of disciplinary research is the existence of an ontological connection between the disciplines. In my case, the target system provides this ontological connection. ${ }^{31}$

In summary, Schmidt (2011) and Thorén and Persson (2013) advocate a problem-oriented approach to interdisciplinary research. Although there are important differences between the cases that they present and mine, their frameworks provide an understanding of what problems are and how disciplinary perspectives interact through problems. Their accounts show, for example, that much of the interdisciplinary epistemic work already goes into constructing an interdisciplinary problem definition. Instead of integration of theories and concepts of the perspectives in order to produce new theories or concepts (or even create a new discipline), the conception of interdisciplinarity that I defend here, like Schmidt's and Thorén and Persson's revolves around problem-solving concerning a

31 An important difference between my case and those that Thorén and Persson present is the interaction between the disciplinary perspectives. In their sustainability science cases, one discipline comes up with a problem, which is then solved by the other discipline without much interaction between the two disciplines, whereas in my case, there is an ongoing interaction between the different disciplinary perspectives. 
target system. The disciplines interact over a problem, where each perspective contributes to finding a solution, without blending or merging with the other disciplines. Rather, it is exactly because the perspectives have their distinct methods, content and values that each can make a valuable contribution that others cannot, and integrating them would weaken this because it reduces the internal coherency of the perspectives. From this problem-oriented understanding of interdisciplinarity, I will turn to my understanding of interdisciplinary expertise.

\subsection{Interdisciplinary expertise}

Important for understanding interdisciplinarity is to realize that disciplinary experts will 'look at' the target-system through their disciplinary perspective. They will 'see' or 'filter-out' aspects of the target system that can be dealt with in their own discipline. Experts involved in interdisciplinary work have to go back and forth between the disciplinary perspectives of all specialisms involved to promote (scientific) reasoning processes that are necessary for the development (turning the initial-undesired state into the goal state) of the target system and its components (i.e., the MRI machine, the patient and his/her condition, the software and clinical practice) to produce a solution that is coherent and consistent with each perspective involved. In other words, these professionals should have the ability to perform specific epistemic activities: the cognitive ability to connect, translate and establish links between disciplinary knowledge of aspects of the target system, not as in a jig-saw puzzle, but by taking into account that knowledge is generated from within disciplinary perspectives. It involves the cognitive ability to deal with the fact that 'knowledge' generated by specific disciplines is indelibly entangled with basic concepts, methods, technologies and specific ways of measuring, reasoning and modelling of that disciplinary perspective.

In the example of the interdisciplinary research project, I described my professional technical medicine role of being a 'connector' between the disciplinary perspectives, and claimed that my interdisciplinary expertise enabled me to perform epistemic tasks involved with interdisciplinary problems solving, such as using the different disciplinary perspectives to reason about the target system, to switch between the disciplinary perspectives, and to reflect on them. My aim here is to provide a more detailed account of interdisciplinary expertise.

To begin with, the concept 'interdisciplinary expertise' seems to be internally incoherent, because as laid out at the beginning of this chapter, expertise and learning a disciplinary 
perspective is usually associated with being immersed - socially and/or intellectually - in a scientific community or discipline. Fleck and Kuhn both emphasize the role of education for the establishment and continuation of a thought collective or scientific community: it is how one becomes a member of such a community and in turn is able to develop scientific knowledge. Mößner (2011) argues that in both Fleck's and Kuhn's view, “education becomes more of a kind of indoctrination than guidance to voluntary and reflective belief formation" (Mößner 2011, p. 364). Collins and Evans (2007) argue that the tacit knowledge required to become an expert is gained by being present in the practice, in the same way as children learn to speak a language by being around people who speak that language. Conversely, I have put emphasis on the role of disciplinary perspectives and the ability to recognize the role of the disciplinary perspective in one's own approaches in professional practice, as well as the ability to understand the workings of disciplinary perspectives of experts educated in other disciplines, especially, when collaborating in interdisciplinary problem-solving. A closer look at the expertise involved in interdisciplinary work is therefore necessary.

One of the few papers (Goddiksen and Andersen 2014) dealing with interdisciplinary expertise refers to the two levels of expertise that Harry Collins and Robert Evans (2007) put forward in their "skill-based view" of expertise (also see Chapter 2). Contributory expertise is defined with respect to a specific scientific domain, as "the ability to make a significant contribution to the development of the practice of the domain," (Goddiksen and Andersen 2014, p.6). Interactional expertise is (in Goddiksen and Andersen's words) "some degree of familiarity with the concepts used in a domain while not necessarily having the further skills needed to perform experiments and detailed mathematical manipulations" (ibid, p.6). According to Goddiksen and Andersen, interdisciplinary expertise involves the abilities to communicate across multiple disciplines and to integrate multiple disciplines. Therefore, in their account, interdisciplinary expertise requires to develop interactional expertise in the relevant domains in order to be able to communicate across domains. In addition, to be able to integrate domains requires developing relevant meta-reflective skills: "skills in critically reflecting on the problem-solving practices that a person is most familiar with" (ibid, p. 4). These include general critical thinking skills, but also skills in identifying and articulating assumptions and standards implicit in problem-solving practices.

Andersen and Goddiksen thus give a first indication of what kinds of skills are required for interdisciplinary research. However, I disagree with their emphasis on acquiring interactional expertise, because on their account this does not seem to go beyond understanding of theories, concept and vocabulary, whereas I argue that interdisciplinary expertise also 
involves being able to do something (i.e., perform epistemic activities, cf. Chang 2012, 2014) with these disciplinary perspectives. In Collin's and Evan's framework, doing something with a discipline is limited to contributory experts. The type of expertise needed for the kind of interdisciplinary research I envision here involves making contributions as well, but to problems external from a specific discipline. Hence, an account of interdisciplinary expertise implies shifting the attention from the content of the disciplines (theories, concepts, etc.) to the epistemic activities that an interdisciplinary expert should be able to perform in order to come up with solutions that effectively combine aspects from multiple disciplines to solve interdisciplinary problems and the cognitive skills that allow them to do this. In this chapter, I will argue that the ability to adequately deal with disciplinary perspectives is crucial to interdisciplinary expertise.

I distinguish two situations in which professionals engaged in disciplinary expertise need to have interdisciplinary expertise: disciplinary experts working in interdisciplinary research projects and 'interdisciplinary professionals' such as professionals in technical medicine whose task is to connect disciplines by facilitating interactions between disciplinary experts and their perspectives in view of a problem. The interdisciplinary expertise of disciplinary experts and interdisciplinary professionals is not fundamentally different, but gradual: an interdisciplinary expert has more interdisciplinary expertise than a disciplinary expert engaged in interdisciplinary research, but does not necessarily have disciplinary expertise. In the example above I show that interdisciplinary expertise involves the ability to effectively combine multiple disciplines by being able to go back and forth between several disciplinary perspectives and use the epistemic resources that they provide. The epistemic resources of a discipline include its methodologies, instruments, modelling practices and theoretical insights, and epistemic activities in a discipline require the ability to utilize these epistemic resources in epistemic tasks, which I have loosely indicated as to reason about the target system in light of the specific problem at hand. Interdisciplinary experts, therefore, have to master multiple disciplinary perspectives (both knowledge and skills) to the extent that they are able to use them for thinking about the target system, i.e., in performing epistemic activities, rather than only translating or communicating across disciplines. Working within real-world problem-contexts - rather than aiming at integrating scientific disciplines for the sake of science - also requires that interdisciplinary experts are able to evaluate what aspects of a discipline are relevant, and which aspects (or details) can be neglected. Additionally, it requires being able to recognize to what extent one's own grasp of a discipline is sufficient or whether collaboration with experts in other disciplines is needed, which then requires the ability of interdisciplinary collaboration as illustrated in the case above. 
As I have shown with the case above, the appropriateness of an interdisciplinary solution is judged, firstly by referring to its purpose, in other words, the problem it aims to solve (Chang 2012, 2014; Thorén and Persson 2013). Additionally, the interdisciplinary solution should be consistent with each disciplinary perspective, in the sense that it cannot conflict with key theories, methodologies, commonly accepted use of instruments, epistemic values or scientific ideals of these disciplinary perspectives. Therefore, to acquire interdisciplinary expertise, experts should know for each disciplinary perspective what aspect of the target system can be understood through it, using what methodologies and instruments, how this is modelled and what are commonly accepted theories and concepts about the target system. However, humans are cognitively limited, and the point of interdisciplinary collaborations is a division of intellectual labour (MacLeod 2018). The issue I aim to address in this chapter is finding a balance between requiring in-depth disciplinary expertise in multiple disciplines and insufficient understanding of other disciplines. Therefore, I aim to come up with an account of interdisciplinary expertise that respects the cognitive limits of experts. Central to my account is the idea that (a) the cognitive challenge of interdisciplinary collaborations for the experts involved is that each expert approaches the interdisciplinary problem from their own disciplinary perspective; (b) the notion of disciplinary perspectives can facilitate communication between disciplinary experts, without requiring each to become specialist in all fields; and (c) dealing with disciplinary perspectives in this very way requires so-called metacognitive skills. In short, an interdisciplinary professional, such as a professional in technical medicine, needs to have a high level of interdisciplinary expertise. However, he or she does not need to become a disciplinary expert in each discipline involved, but still needs to have some knowledge of the content of the disciplines involved, as well as the mentioned metacognitive skills needed to use disciplinary perspectives in thinking about an interdisciplinary problem. Conversely, disciplinary experts working in interdisciplinary projects have a high level of disciplinary expertise and additionally, need at least some interdisciplinary expertise.

\subsection{Teaching interdisciplinarity - technical medicine}

In the final paragraph of this chapter, I will turn to a case of interdisciplinary teaching to show how my understanding of interdisciplinary expertise can help overcome the epistemological difficulties that often play a role when teaching interdisciplinarity to students, i.e., the case of a new interdisciplinary educational program technical medicine.

In response to a rapid increase of innovative medical technologies that (will) find their way into clinical practice, a new kind of medical professional has been introduced. This 
professional - the technical physician - is specialized in applying, adapting and developing complex medical technologies in the diagnosis and treatment of individual patients (Groenier et al. 2017; Miedema 2015), which requires this professional to have adequate technological knowledge as well as insight in how to solve medical-technical problems. This professional, therefore, needs to combine two disciplines, and is therefore characterized as an interdisciplinary expert in the sense suggested above. As has already been argued, it cannot be expected that an interdisciplinary expert is an expert in both domains. As such, technical physicians are not doctors who are involved in the patient's complete medical pathway (but often in one particular stage in the diagnosis or treatment of a patient), neither are they engineers that develop or design medical devices. ${ }^{32}$

Technical medicine is an interdisciplinary profession. The educational program aims at experts who are able to systematically apply and combine knowledge from different disciplines —including medical sciences, natural sciences and technology — to solve problems concerning diagnosis and treatment of individual patients. The curriculum specifically intends to 'synthesize' technological and medical knowledge so that students learn to come up with solutions to clinical problems by integration of technology and medicine throughout the program (Miedema 2015, pg. 10). Technical medicine as a field or profession does not aim at integrating (in the sense of integrating theories and concepts) technical and medical disciplines. Rather, like sustainability in Thorén and Persson's account, technical medicine is a problem-oriented profession that collaborates with, and bridges between disciplinary experts (from medical sciences and biomedical engineering in particular) to solve problems that are specific to technical medicine (i.e., medical problems that require technological solutions). Therefore, students in technical medicine need to learn to pick out the relevant disciplinary perspectives for a specific problem and learn to collaborate with experts from these fields to solve this problem. As I have argued above, this also means that they are aware that disciplinary experts (with whom they will work) will 'look at' the target-system through their disciplinary perspective and 'see' or 'filter-out' only those aspects of the target system that can be dealt with in their own discipline. Technical physicians have to go back and forth between the disciplinary perspectives of doctors and technologists to promote an interdisciplinary collaborative (scientific)

32 An example of what technical physicians do in clinical practice is hyperthermia treatment. Hyperthermia treatment involves a technology that uses microwaves to locally heat tumor tissue in order to improve the effect of radiotherapy or chemotherapy. A specialized technical physician collaborates with the physician to perform this technologically complex treatment and to analyze the effects on individual patients. This and other examples can be found on http://www.nvvtg.nl/technische-geneeskunde/cases/ (in Dutch) 
reasoning process that is necessary for the development, implementation and uses of medical-technical systems, such as technologies for diagnosing and treating patients. The expertise of technical physicians is thus fundamentally different from the expertise that disciplinary professionals have, as it is not by means of immersion in a community that the expertise is obtained and evaluated. However, it is not fundamentally different from the interdisciplinary expertise that all professionals engaged in interdisciplinary work (should) have. Rather, technical physicians are specialized in solving a certain type of interdisciplinary problems (medical problems that require technological solutions) and are familiar with the disciplines involved to such an extent that they can take the role of 'connector' as I have described in the case. As a 'connector' technical physicians are able to build connections in interdisciplinary collaborations thereby facilitating profound interactions between and contributions by disciplinary experts, which might not succeed if left to disciplinary experts themselves.

If interdisciplinary expertise is fundamentally different from disciplinary expertise, learning this expertise is fundamentally different. How can students learn interdisciplinary expertise? In the analysis of the case, I have argued that interdisciplinary expertise requires higher-order cognitive skills at two levels. 'Level 1 higher-order cognitive skills' involved those skills that allow performing epistemic activities from within disciplinary perspectives, such as mathematics, research methods and problem-solving as well as the switching between them. For this, students in technical medicine should learn to reflect on their own learning, to be able to identify what they need to know and learn and where the gaps in their own and someone else's knowledge are. ${ }^{33}$ Therefore, I have argued that interdisciplinary experts also have a specific type of meta-cognitive skills that I called 'level 2 higher-order cognitive skills'. This involved the ability to reflect on the disciplinary perspectives to identify aspects as outlined in Table 1. In this manner, students learn to see differences and similarities between disciplinary perspectives and may start to understand the challenges of collaborative interdisciplinary problem-solving. My central claim in this chapter is that a first step to acquiring these higher-order cognitive skills is to make students aware of the role of disciplinary perspectives in doing scientific research that aims at problem-solving, which amongst other things involves making students aware that knowledge produced in scientific research is shaped by the disciplinary perspective. For

33 In addition, some other professional (social and personal) skills for interdisciplinary collaborations are necessary as well, for example; flexibility, communication skills, having an overview over all relevant disciplines (rather than focusing on depth), to be innovative, independent and to have an open, enthusiastic and social attitude. 
example, students can be trained to recognize how a disciplinary perspective shapes what experts within a certain discipline 'see' when they study a certain target-system. In the case I illustrated how this works in practice. This can be made explicit to students when teachers illustrate how they deal with a target-system from their disciplinary perspective, in other words, what questions they ask, how they perform measurements, how they draw conclusions about the target system, etc. Students can do similar exercises by listing the general aspects of disciplines they 'use' to solve a certain technical-medical problem. Additionally, interdisciplinary expertise not only involves understanding how different disciplinary experts 'see' a target system but also how they generate knowledge about the target system, i.e., how they do research. Therefore, teaching interdisciplinary expertise requires making explicit how disciplinary experts craft a 'picture' or 'model' of the target system. A recently published general methodology for constructing models can help in learning how to teach this in a systematic manner (Boon forthcoming).

\subsection{Conclusion}

In this chapter, I have argued that the abilities to perform epistemic activities, and thus to construct and use scientific knowledge, are shaped by disciplinary perspectives. These disciplinary perspectives can be seen as a coherent set of aspects such as goals, phenomena of interest, objects of investigation, ways of modelling, theories and concepts, methodology and instruments, and epistemic values and scientific ideals (as listed in Table 1). Disciplinary perspectives shape how experts 'see' a target system. It means that they first pick-out and define specific aspects of the broader target system as the object of their investigation. Additionally, it shapes the way in which experts think about investigating these aspects (also called the phenomena studied) in the sense that the disciplinary perspective guides in choosing the methodology and instruments used in the investigation; the ways of modelling these phenomena (e.g., mathematical, causal-mechanistic, statistical, morphological, etc.); the key concepts and theories; and scientific ideals and epistemic values typical of the discipline. Through an example of an interdisciplinary research project - the development of a new imaging tool for clinical practice - I have illustrated what these disciplinary perspectives mean in practice.

After that, I have also used the notion of disciplinary perspective to gain a better understanding of interdisciplinarity. I claim that traditional conceptions of interdiscipinarity usually focus on interdisciplinary research within science, which often aim at the integration of theories and concepts. Rather, my focus is on interdisciplinary problem-solving. I argue that in the case I present, the researchers do not aim at the integration of theories 
or concepts, nor is the integration of theories and concepts necessary in scientific research aimed at real-world problem-solving. Rather, experts enter into an interdisciplinary collaborative reasoning process, where each expert, from their own disciplinary perspective, contributes in reasoning about phenomena indicated by other disciplinary perspectives. In that sense, disciplines interact to solve a specific problem. In interdisciplinary collaborations of experts, disciplinary perspectives on the problem take turns, building on each other in gradually developing an interdisciplinary solution. In this collaboration, each expert performs epistemic activities that suit with his or her disciplinary perspective, and experts can understand each other's contributions by understanding how disciplinary perspectives work and having at least some understanding of the characteristic features of the disciplinary perspectives involved. If interdisciplinary collaboration works properly, disciplinary experts will be able to effectively go back and forth between different disciplinary perspectives in order to use them in reasoning about the target system and effectively combine them in a solution that is internally coherent as well as consistent with all perspectives involved. The solution is evaluated in reference to the overall purpose of the interdisciplinary project as well as its consistency with each relevant perspective. Hence, the outcome of interdisciplinary research is not a blend of each discipline, where each discipline is watered-down by the others and the outcome does not quite fit the (epistemic criteria of) any of the disciplines. Rather, good interdisciplinary research makes use of the fact that the contributing disciplines are well-established and have developed internal validity. In this approach, experts have the epistemological responsibility to ensure that their own contributions as well as the overall interdisciplinary outcome are consistent with their own discipline. Reaching this involves more than a straightforward process in which pieces of knowledge from different disciplines are fitted together like a jig-saw puzzle: having such a collaboration requires to reflect on epistemic values, methodology and content that may be different for each disciplinary perspective, in order to identify gaps, opportunities and conflicts. Interdisciplinary collaboration, therefore, requires a specific type of expertise, that I call interdisciplinary expertise. I use the case of teaching technical medicine to illustrate that developing this interdisciplinary expertise involves developing cognitive skills at two levels: those cognitive skills that allow one to perform epistemic activities from within disciplinary perspectives (level 1), and higher-order cognitive skills (level 2) that involve reflecting on disciplinary perspectives and how they shape knowledge use and construction. With these suggestions, I have not given a specific proposal for curriculum design for interdisciplinary learning, but have provided basic strategies for acquiring the higher-order cognitive skills required for interdisciplinary problem-solving. 


\section{References}

Andersen, H. (2016), 'Collaboration, interdisciplinarity, and the epistemology of contemporary science', Stud Hist Philos Sci, 56, 1-10 DOI: 10.1016/j.shpsa.2015.10.006.

Boon, M. (2017), 'An engineering paradigm in the biomedical sciences: Knowledge as epistemic tool', Progress in Biophysics and Molecular Biology, 129, 25-39 DOI: 10.1016/j.pbiomolbio.2017.04.001.

Boon, M. (under review). 'Epistemological Issues of Interdisciplinary Scientific Research for Real-world Problem-solving.'

Boorse, C., (1975), 'On the distinction between disease and illness,' Philosophy and public affairs, 5: 49-68

Brown, M. J. (2009), 'Models and perspectives on stage: remarks on Giere's Scientific perspectivism', Studies in History and Philosophy of Science Part A, 40 (2), 213-20 DOI: 10.1016/j.shpsa.2009.03.001.

Chandarana, H. et al. (2012), 'Diffusion-Weighted Intravoxel Incoherent Motion Imaging of Renal Tumors with Histopathologic Correlation', Investigative Radiology, 47 (12), 8 DOI: 10.1097/RLI.0b013e31826a0a49.

Chang, H. (2009), 'Ontological principles and the intelligibility of epistemic activities.', in H. W. De Regt, S. Leonelli, and K. Eigner (eds.), Ontological principles and the intelligibility of epistemic activities (Pittsburgh: Ontological principles and the intelligibility of epistemic activities) ISBN: 9780822971245

Chang, H. (2012), Is Water H2O? - Evidence, Realism and Pluralism (Boston Studies in the Philosophy and History of Science; Dordrecht: Springer) ISBN: 978-94-007-3931-4.

Chang, H. (2014), 'Epistemic Activities and Systems of Practice: Units of Analysis in Philosophy of Science After the Practice Turn.', in L. Soler, et al. (eds.), Science After the Practice Turn in the Philosophy, History and Social Studies of Science. (London: Routledge), 67-79 ISBN: 978-0415722957

Collins, H. and Evans, R. (2007), Rethinking Expertise (Chicago, London: The University of Chicago Press) ISBN: 978-0-226-11360-9.

Feng, Q. et al. (2016), 'Usefulness of diffusion tensor imaging for the differentiation between low-fat angiomyolipoma and clear cell carcinoma of the kidney', SpringerPlus, 5 (12) DOI: 10.1186/s40064-0151628-x.

Fleck, L. (1979), Genesis and development of a scientific fact (Chicago: University of Chicago Press) ISBN: 9780226253251.

Giere, R.N. (2006), Scientific perspectivism (University of Chicago Press) ISBN: 9780226292137.

Goddiksen, M. and Andersen, H. (2014), 'Expertise in Interdisciplinary Science and Education', (philsciarchive.pitt.edu/11151/).

Groenier, M., Pieters, J. M., and Miedema, H. A. T. (2017), 'Technical Medicine: Designing Medical Technological Solutions for Improved Health Care', Medical Science Educator, 27 (4), 621-31 DOI: 10.1007/ s40670-017-0443-z.

Huutoniemi, K. et al. (2010), 'Analyzing interdisciplinarity: Typology and indicators', Research Policy, 39 (1), 79-88 DOI: 10.1016/j.respol.2009.09.011.

Khushf, G. (1999), 'The Aesthetics of Clinical Judgment: Exploring the Link between Diagnostic Elegance and Effective Resource Utilization', Medicine, Health Care and Philosophy, 2 (2), 141-59 DOI: 10.1023/a:1009941101276.

Klein, Julie Thompson (2010), 'A Taxonomy of interdisciplinarity', in Robert Frodeman (ed.), The Oxford Handbook of Interdisciplinarity (Oxford: Oxford University press), 15-30 ISBN: 978-0-19-923691-6

Kuhn, T.S. (1970), The Structure of Scientific Revolutions, Second Edition, Enlarged, ed. O. Neurath (International Encyclopedia of Unified Science; Chicago and London: The University of Chicago Press.) ISBN: 0-22645803-2. 
Kuhn, T.S. (1977), The Essential Tension. Selected studies in scientific tradition and change. (Chicago and London: University of Chicago Press.) ISBN: 978-0226458069.

MacLeod, M. (2018), 'What makes interdisciplinarity difficult? Some consequences of domain specificity in interdisciplinary practice', Synthese, 195 (2), 697-720 DOI: 10.1007/s11229-016-1236-4.

Miedema, H.A.Th. (2015), 'Arts en Ingenieur: and ever the twain shall meet', (University of Twente).

Mößner, N. (2011), 'Thought styles and paradigms-a comparative study of Ludwik Fleck and Thomas S. Kuhn', Studies in History and Philosophy of Science Part A, 42 (2), 362-71 DOI: 10.1016/j.shpsa.2010.12.002.

Nersessian, N.J. and Patton, C. (2009), 'Model-based reasoning in interdisciplinary engineering', in A. Meijers (ed.), Handbook of the Philosophy and Engineering Sciences (Amsterdam: Elsevier), 687-718 ISBN: 9780-444-51667-1

Rheinheimer, S. et al. (2012), 'Investigation of renal lesions by diffusion-weighted magnetic resonance imaging applying intravoxel incoherent motion-derived parameters--initial experience', Eur J Radiol, 81 (3), e310-6 DOI: 10.1016/j.ejrad.2011.10.016.

Schmidt, J. C. (2011), 'What is a problem?: On problem-oriented interdisciplinarity', Poiesis \& Praxis, 7 (4), 249-74 DOI: 10.1007/s10202-011-0091-0.

Schön, D. (1983), The Reflective Practitioner - How professionals think in action. (New York: Taylor \& Francis Ltd.) ISBN: 978-0465068784.

Thorén, H. and Persson, J. (2013), 'The Philosophy of Interdisciplinarity: Sustainability Science and ProblemFeeding', Journal for General Philosophy of Science, 44 (2), 337-55 DOI: 10.1007/s10838-013-9233-5.

Toulmin, S. (2001), Return to Reason (Cambridge, MA \& London: Harvard University Press) ISBN: 9780674012356.

van Baalen, S. et al. (2017), 'Intravoxel incoherent motion modeling in the kidneys: Comparison of mono-, bi-, and triexponential fit', J Magn Reson Imaging, 46 (1), 228-39 DOI: 10.1002/jmri.25519.

van Baalen, S. et al. (under review) 'Mono, bi- and tri-exponential diffusion MRI modelling for renal solid masses and comparison with histopathological findings'

van der Bel, R. et al. (2017), 'A tri-exponential model for intravoxel incoherent motion analysis of the human kidney: In silico and during pharmacological renal perfusion modulation', European Journal of Radiology, 91, 168-74 DOI: 10.1016/j.ejrad.2017.03.008. 


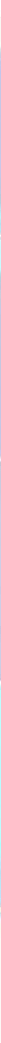


Developing an imaging tool for clinical practice

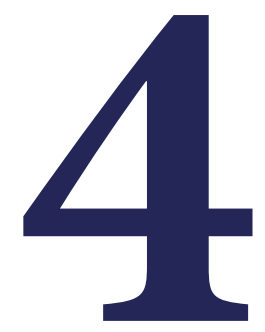

The empirical data in this chapter was collected during a study visit at Medical Humanities at the University of Sheffield.

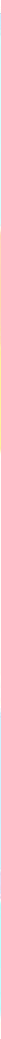




\section{Abstract}

In the past decades research into medical imaging modalities such as X-ray computed tomography (CT), magnetic resonance imaging (MRI) and ultrasound has rapidly increased, bringing forth many new outlooks on the human body. However, only a small fraction of these developments have found their way into clinical practice. In this chapter, I argue that this is because medical technologies are often developed with little regard for the specificities of the clinical practices at which these technologies are aimed. I will defend that developing an imaging tool for medical practice requires intensive collaboration between medical imaging developers and their (future) users - medical experts. I present a case study of a multidisciplinary research team involved in the development and introduction of a new and innovative MRI tool that has not been adopted into daily clinical practice yet, called hyperpolarized gas imaging. By zooming in on the actual reasoning processes in this team while they are discussing the images, I point out why interdisciplinary collaborative reasoning between experts with different disciplinary perspectives are imperative for the successful development of a clinical imaging tool. I show that it is not straightforwardly obvious what can be seen in a new medical image. Rather, establishing what is 'seen' and what the image can 'tell' about the patient's condition results from a search process in which experts search for something that is reliable, interpretable, useful and relevant for the specific context. In this search process, team members enter into multiple cycles of abduction - deduction - induction, in the sense of C.S. Peirce (1908), and coordinate and adapt their interpretations and contributions in such a way that they feed to each other. Suggestions and questions from the clinicians help the engineer to articulate follow-up research, whereas the interpretations by the radiologists help the clinician to better understand his patient's condition. This brings forth a collaborative and interdisciplinary reasoning process. The imaging technique, what and how it represents, and its interpretation (in the context of a specific patient population) are interdependent and therefore need to be established in a mutual interaction. In an iterative process, clinicians come to relate aspects of the image to aspects of their patients, while developers come to understand which features of the image are valuable to clinicians and subsequently tweak the technology so that these are highlighted amongst the abundance of other data. It is through the interaction of the technology, the image, and the experts' input that the representation and interpretation of an image become established. 


\subsection{Introduction}

In the past decades research into medical imaging modalities such as X-ray computed tomography (CT), magnetic resonance imaging (MRI) and ultrasound has rapidly increased, bringing forth many new outlooks on the human body. Quantitative imaging, three dimensional (3D) renderings and functional methods (e.g. evaluating perfusion, ventilation or flow) showed promising results in the assessment of a wide range of pathologies, but only a small number of these innovations have found their way into clinical practice. Despite efforts to involve clinicians in the introduction of new imaging tools and the assessment of the clinical value, the main challenge of medical imaging development remains the adoption of new imaging tools in day-to-day clinical practice.

An example of this relates to the case in Chapter 3. Diffusion-weighted magnetic resonance imaging (DW-MRI) that probes the random movement of water molecules ('Brownian motion') was first introduced in 1984 as an additional biophysical parameter that can be determined by MRI technology. This has grown into a vibrant research field. Within this research field, a range of new parameters quantified using diffusion MRI to characterize a range of tissue characteristics throughout the human body have been developed, in addition to an array of models for accurate and precise modelling of the diffusion signal, and number of software packages to investigate and visualize diffusion. The research project in Chapter 3 is an example of an attempt to develop a clinical application of diffusion-weighted MRI, but the outcome of this research has not yet reached clinical practice. Current clinical use of diffusion MRI is limited to the diffusion parameter that was first introduced and is the least complex, i.e., the apparent diffusion coefficient (ADC), which is a measure for the magnitude of diffusion and most often used to detect a stroke in the brain.

The issue that developments in biomedical science and technology (the 'lab bench') are not finding their way into clinical practice (the patient's 'bedside') has been raised in the wider context of biomedical research. As a response, 'translational research' has become a buzzword in the field, and the term is used for a large set of research activities, from biomarker development to clinical studies of a new medical tool. The abundance of this term in policy reports, the titles of academic research papers, scientific journals, and research institutes is indicative of how important 'translation' is taken to be (van der Laan and Boenink 2015). In the biomedical research context, policy-makers, funding institutions, researchers and physicians perceive a gap between fundamental or basic research and clinical application (Butler 2008). In this chapter, I aim to address a specific cause of the gap between basic technological research and clinical application, which is the 'gap' 
between the expertise of (medical imaging) technology developers and the expertise of their users, causing that medical technologies are often developed in isolation, i.e., disregarding the specificities of clinical practices at which these technologies are aimed. I will defend the view that developing an imaging tool for medical practice requires intensive collaboration between experts from both sides, whereas an assumption underlying current research and development practices is that innovation takes place in different stages, which starts with technology development and ends with adopting the technology into clinical practice. In this view, different experts play bigger or smaller roles in different phases of innovation. Being able to provide (additional) information on patients and their condition is an important requirement for the adoption and acceptance of new imaging tools. This is what medical technologies aim to offer. Why then are imaging technologies often not adopted in clinical practice? A crucial element of this gap in medical imaging development revolves around an epistemological question: how can images be a source of information for diagnosis and treatment decisions in clinical practice? In this chapter, I focus on an epistemological analysis to explain why the construction of images from technologically generated signals (such as in diffusion MRI) requires expertise from both the developers of technology and the users of these images - which implies that the development of imaging technologies requires interdisciplinary research projects in which the collaboration between technological and clinical practice is crucial. Conversely, in most current research projects aiming to develop imaging technology for clinical practice, this is not the case - clinicians are only included at a later stage after the technology has been developed.

In Chapter 3, I have argued that experts generate knowledge from a specific disciplinary perspective that shapes how they see and investigate aspects of a target system. I presented an example of an interdisciplinary research project aimed at the development of a new imaging tool for clinical practice, in which clinicians actually take part. In the analysis of that case, I aimed to illustrate that multiple disciplinary perspectives are involved, and how these perspectives are linked. In this analysis, it was shown that reasoning within one perspective provides input in another, as an iterative process that eventually results in an interdisciplinary solution, without actual integration of theories or disciplines.

In the current chapter, I will analyse another case, in which I zoom in on actual interdisciplinary collaborative reasoning processes in an interdisciplinary research project in order to point out why an exchange between experts with different disciplinary perspectives is imperative for the successful development of a clinical imaging tool. I have studied a multidisciplinary research team involved in the development and introduction of a new and innovative MRI tool that has not been adopted into daily clinical practice yet, called hyperpolarized gas imaging. This team consists of a clinician (a consultant respirato- 
ry clinician), a radiologist (specialized in thorax-imaging), a radiographer, and an MRI engineer. A team meeting in which they discuss the first two sets of hyperpolarized gas images from patients with lung disease that the clinician has trouble diagnosing has been video-recorded. I zoom in on the interdisciplinary collaborative reasoning processes in interactions between these professionals who collaborate to find out how to interpret the images in view of how this new tool may contribute to the diagnosis and treatment practice of the clinician (the consultant respiratory clinician).

In the philosophy of science, there are several accounts of how images can function as evidence providers, which basically analyse how images are representations of the imaged object. For example, several authors assume that the evidentiary status of images depends on the extent to which technology provides a direct, causal link between the object and the resulting image (e.g., Hacking 1982; Perini 2012) while other authors focus on the interaction (hermeneutics) between the image, the user of the image, and the resulting interpretation (Friis 2015; Rosenberger 2009; Suárez 2004). These contrasting views on how images and visualizations provide evidence align with the two sides of the 'gap' between engineers and clinical practice, and with the two types of research in each of these fields. In their own research, engineers tend to focus on providing evidence for the image being a faithful representation of what is being imaged (e.g., organ morphology or tissue structure), which basically consists in aiming at a direct, causal link between object and image. The required subsequent research steps are concerned with the interpretation of images by clinicians (and often expected to be performed by medical experts): first developing images (or 'maps') that are easily accessible by clinicians and after that establishing a correlation between an imaging result and a specific pathology, which agrees to the hermeneutic interpretation that focuses on the interaction between image and user (e.g., clinicians). Although my analysis in terms of two philosophical views helps to clarify the gap between approaches in the two research practices involved in developing new medical imaging technologies, it does not yet bridge the 'gap' between basic research and clinical application, nor does it explain why the gap needs to be bridged.

Through philosophical analysis of (video-recorded) interactive reasoning processes in interdisciplinary research aiming at the development of an imaging technology, I aim to uncover the epistemology of this type of interdisciplinary collaborations. This includes aiming to understand if and in what way knowledge is used and produced in the context of the development of medical imaging tools. There are two ways in which knowledge plays a role. Firstly, the development of technology involves scientific research, which uses and produces knowledge. Secondly, clinicians will use the imaging tool as evidence providers to produce knowledge about patients and their conditions. Epistemological questions that 
I ask in my philosophical analysis are, for example: What does it mean for an image to serve as evidence in diagnosis and treatment decisions? How can different experts bring their specific expertise together to figure out the epistemic value of the images, and how do these images gain meaning in a specific medical context? How does expertise (knowledge, skills, reasoning methods) add to this process? And finally, how can the quality of this process be warranted such that the images can be considered a reliable source of information for the clinical decisions made on their basis?

Combining empirical material with philosophical literature, I will argue that the 'evidence value' of a new imaging tool is not only determined by the technology, nor is it purely a product of the (social) interaction between a clinical expert and the image. Hence the image as a diagnostic tool cannot be established by only proving the correlation of the image to the body's physiology. Based on my analysis, I will substantiate the following claims:

1. A medical image visualises an abundance of information from which it is not straightforwardly obvious how the image relates to the patient. Rather, establishing what is 'seen' and what the image can 'tell' about the patient's condition results from a search process in which experts search for something that is reliable, interpretable, useful and relevant for the specific context.

2. The imaging technique, what and how it represents, and its interpretation (in the context of a specific patient population) are interdependent and therefore need to be established in a mutual interaction. Clinicians come to relate aspects of the image to aspects of their patients, while developers come to understand which features of the image are valuable to clinicians and subsequently tweak the technology so that these are highlighted amongst the abundance of other data. It is through the interaction of the technology, the image, and the experts' input that the representation and interpretation of an image become established in an iterative rather than linear development process.

In Paragraph 4.2., I will present an overview of the philosophical literature on images. After that, in Paragraph 4.3., I describe the research that is done by a multidisciplinary team consisting of a clinician, a radiologist, a radiographer, and an engineer collaborating on the development of a new MRI imaging technique for clinical practice. Finally, in Paragraph 4.4. and 4.5., I present my analysis of their interdisciplinary collaborative reasoning concerning the patient, the images, the technology and the interpretations. Through fragments of the discussions between the team members, I illustrate in what sense their reasoning is collaborative. 


\subsection{Philosophical literature on imaging and scientific representa- tion}

The philosophical literature deals with imaging and scientific representation in several ways. In the philosophy of science, authors focus on the question of justification of the use of images as evidence for scientific claims. Usually, this question revolves around representation: images represent certain aspects of the world in one way or another, which usually relies on structural similarities between objects and images (Giere 2006; Hacking 1981), or on the causal relationship between objects and images provided by the imaging technology (Perini 2012). Both accounts, however, neglect the epistemological challenges of generating these structural similarities and establishing such causal relationships. Other authors in the philosophy of science focus on the interpretation by pointing out that crucial aspects of scientific representations are the kind and ease of inferences they allow (Kulvicki 2010; Suárez 2004), which, however, does not explain how an image becomes meaningful to the user as this requires taking into account epistemic aspects such as background knowledge, expertise and experience. Conversely, the phenomenological literature also focuses on the interpretation of images, but explores this in terms of gestalt and tacit knowledge, thereby explaining how an image becomes meaningful to a user (Friis 2015; Rosenberger 2009). However, these phenomenological accounts are largely individual, only explaining how images become meaningful to the person interacting with the images, and cannot account for the more collaborative processes in which, I claim, these images gain meaning in a specific medical context.

\subsubsection{Images as evidence for (scientific) claims}

The first question about imaging that philosophers aim to answer is whether and how images are representations of the object of imaging. Ian Hacking (1981) attempts to answer this question for observations made using a microscope in Do we see through a microscope. A microscope does not produce images in exactly the same way as other imaging technologies. Using a microscope, researchers enter in a direct interaction with the object and the technology, allowing them to adjust the view and manipulate the specimen. Other (medical) imaging technologies usually produce static images of objects to be viewed at a later time, thus omitting the possible interactions between the user and the technology (one notable exception in the clinic is ultrasound). This difference between microscopes and other imaging modalities seems relevant when one talks of 'seeing through' but Hacking extends his analysis to other types of microscopic imaging such as the electron microscope and the acoustic microscope and asks whether we could speak of 'seeing' in these cases. To do this, he omits the word through: "the micrograph is more to the point: we study photographs taken with a microscope," (Hacking 1981, p. 149). He contrast 
viewing a drawing of a cell he has studied before (case '(I)') with "using a digitized and reconstructed image" (case '(II)') and argues: "Even if I am 'looking at the cell' in case (II), in (I) I am only looking at a drawing of the cell. What is the difference? The important feature is that in (II) there is a direct interaction between a wave source, an object and a series of physical events that end up in an image of the object.[...] in case (II) we have a map of interactions between the specimen and the imaging radiation"(Hacking 1981, p. 150). If this map is a good one, Hacking considers the use of these technologies as 'seeing with a microscope' (although he admits that this might be a liberal extension of the notion of seeing). We may then ask how we assess whether a technology produces a good map. When is it a 'truthful' map? In Hacking's view, there are two reasons that microscopes produce good maps. First, he mentions that biologists routinely use grids, made to determine the location of specific aspects of a sample. Because these grids have exactly the same structure under the microscope as expected from their fabrication (i.e., drawing and then shrinking), what is seen under the microscope can be considered truthful. Secondly, Hacking argues that different types of microscopes, using different physical principles (e.g., the acoustic microscope, the electron microscope, the polarizing microscope) produce maps in which similar features of a cell are visible. ${ }^{34}$

Ronald Giere (2006) puts forward that, due to differences in physical principles, scientific instruments (including imaging technologies) provide a specific perspective on the world, depending on what aspects of reality an instrument is sensitive to. For example, a computed tomography (CT) scanner detects x-rays, a positron emission tomography (PET) scanner detects positrons, and a magnetic resonance imaging (MRI) scanner detects changes in magnetic fields, thus offering different ways of representing the human body. Both Giere and Hacking argue that images represent the world through structural similarities with the object in the real world. Hacking argues this by means of the shrunken grid that is visible under that microscope. Giere illustrates it metaphorically with an example of maps representing certain structural aspects of a city. However, Giere expands on Hacking by arguing that scientist, being intentional agents, play an active role in the choice of perspectives and the way they draw conclusions from a representation, which he summarizes with the statement: "S uses X to represent W for purposes P" (Giere 2006, p. 60). In this equation, X can be anything from computer-generated images to photographs, diagrams, theories and models. Hence, Giere introduces an approach to scientific representation that is sensitive to the use of images for scientific reasoning,

34 William Wimsatt (2007) in Re-engineering philosophy for limited beings calls this method to establish and test experimental findings by different methods 'robustness.' 
their epistemic purpose. According to Giere, when multiple perspectives (i.e., instruments) provide a (structurally) similar representation of something that is evidence of there being something there, but not in a way that escapes perspectives. Here, Giere differs from Hacking. Hacking may agree with Giere that instruments provide a specific perspective on the target phenomenon. However, according to Hacking, combining the perspectives of various microscopes, and our ability to interfere with cells under the microscope gives us reason to be convinced of the existence of these structures, independent from the methods that are used to visualize them. Conversely for Giere, all knowing in science is perspectival or in other words, we can only know that $\mathrm{Y}$ according to perspective $\mathrm{X}$. For Giere and Hacking, an image does not speak for itself in any straightforward manner: in Giere's account, to 'read' an image, one needs to know the purpose with which the image was produced (what is it meant to represent), and the convention of reading that type of image. For Hacking, 'seeing' and the conviction that the observed objects are real are the product of combining many things, including interactions with the object, a long process of improving the technology, removing aberrations and artefacts, and the "admirable intersections with biochemistry, which confirm that the structures that we discern with the microscope are individuated by distinct chemical properties too," (Hacking 1981, p. 151). Hacking's and Giere's accounts show that images should be analysed in terms of the specific interactions between the object and the imaging technology (i.e., physical interactions), as well as interactions between the image and the observer, thereby providing a detailed understanding of imaging and representation.

Laura Perini (2012) uses images produced by the electron microscope to illustrate her argument that such 'mechanically produced images' - which she defines as "the products of automated imaging techniques involving an interaction between a specimen and imaging technology, which produces a two-dimensional visible array that is printed or screen-displayed" (Perini 2012, p. 154) - convey representational content because there is a causal link with nature: "not just a causal link between specimen and image, but through interpretative practices [in which users interpret the image in terms a specific kinds of concept, i.e., a dental x-ray in terms of teeth], a connection to the representational content of the image" (ibid, p. 168). Perini argues that image interpretation is constrained because automated imaging techniques are selective, sensitive to certain aspects of a specimen and indifferent to others. A link with the specimen persists because of this constraint. For Perini, the evidentiary role of images depends on whether or not there is a causal relationship between the object and the image.

Although Giere, Hacking and Perini acknowledge that technological instruments generating an image of an otherwise invisible object shape the image that is produced by picking 
up on specific physical aspects of a phenomenon and not others, they treat the resulting image as if it is not fundamentally different from images that result from having direct (unmediated) access to the imaged object. For instance, although Giere acknowledges that images are perspectives determined by the instrument used, his map metaphor does not include the role of technology in generating data upon which the image is constructed. Another issue that they pass over is that objects need to be specifically prepared for imaging in order to detect a signal. For example, cells need to be stained in order to make structures inside the cell visible under a microscope. Similar to how Giere, Hacking and Perini treat images, Kelly Joyce (2005) demonstrates that in popular speech MRI is often depicted as providing unmediated access to the physical body, which passes over the role of technicians' and physicians' background knowledge about MRI technology and images. In practice, however, according to Joyce, the use and quality of MRI images are also shaped by choices made by technologists, for example, the parameter settings (such as slice thickness and field of view) of the MRI machine influence the content of the image. Radiologists continuously make decisions on how to interpret certain features of an image in order to translate the images into a written report for the referring physician. And the referring physicians using the report to make clinical decisions about their patients need to combine the findings from MRI with findings from other tests and decide on the relative value of each of these findings. Joyce thus shows that it is not only the technology that shapes the image through its physical interaction with the object but also the professionals involved in the production and use of the images. While Joyce points to the social relations and processes that shape the production and use of MRI images, I will focus on the role of specific epistemological frameworks of users in these mechanisms.

\subsubsection{Interpretation of images}

To deal with the fact that images do not speak for themselves, other authors focus on the interpretation of images. Mauricio Suárez (2004) explains representation in terms of the "capacity of a source to lead a competent and informed agent to a consideration of the target," (Suarez 2002, p. 768) in addition to the representational force of an image, by which he means the image being "informative regarding the target of the representation," (ibid, p. 772). In his inferential conception of representation, the intended use and the skills and level of competence of the user of an image play a crucial role in ensuring valid reasoning based on the source. However, Suárez does not consider how users interpret images, (i.e., how background knowledge and aims informs interpretation) and what it is in images that makes interpretation possible. Kulvicki (2010) provides a first answer to this latter issue by working out the difference between images and graphs, on the one hand, and descriptions and related representations (like lists), on the other. What sets images apart from descriptions, according to Kulvicki, is that "they present information immediately 
across many levels of abstraction,"(Kulvicki 2010, p. 302). This allows agents to isolate the feature of interest and extract the specific required content from the wealth of information that the image represents in many different ways, depending on the agent's purpose. In addition, he argues that the special epistemic place that pictures, images and graphs can hold follows from the fact that "interpreting them mimics the way in which we glean information about our environment perceptually," (ibid, p. 311).

Authors in the phenomenological tradition also focus on the interpretation ('hermeneutics') of images. Jan Kyrre Berg Olsen Friis (2015) analyses the interpretation of images by radiologists in terms of phenomenological hermeneutics, in which perceivers (having background knowledge, experiences, etc.) become part of the hermeneutic circle where the perceiver continuously moves from the parts to the whole. In his view, radiologists' 'seeing' involves gestalt, "something that stands out against a background and enables us to identify patterns," that cannot be understood as a feature of the image itself, but only of the image in interaction with the perceiver (Friis 2015, p. 210). Through recognizing patterns, radiologists attribute meaning to images. According to Friis, interpretation by the perceiver of an image takes place in a pre-conscious and a reflective phase, and this process is shaped by the perceiver's background. Friis argues that visual perception is an embodied skill that is shaped by biology, society, experiences and training that together make up a personal 'horizon'. As a result, different radiologists can 'see' different things in the same image. According to Friis, horizons can also merge in a process of negotiation when several radiologists work together on the interpretation of an image. Robert Rosenberger (2009) argues (building on Ihde 1986) that images are multistable, meaning that they can be interpreted differently by applying a different hermeneutic strategy, a "framework of interpretation and interpretive skills" (Rosenberger 2009, p. 14). He applies this theory to an ongoing debate in neurology, where the two opposing parties interpret the same images differently. Questioning each party's hermeneutic strategy, Rosenberger argues, can suggest further trajectories for research by asking for a more detailed account of the morphologies present in the image according to rivalling theories.

These authors, although coming from different philosophical traditions (philosophy of science, and phenomenology) emphasize that images display an excess of data, whereas authors like Hacking seem to assume that the technology provides a limited amount of data in which the pattern can be recognized in a straightforward way. The challenge of interpretation, however, is not merely identifying a pattern, but also justifying that the pattern represents an aspect of the studied phenomenon. Conversely, Friis and Rosenberger argue that the issue is identifying a pattern or gestalt in the data, through which information from the images becomes available to the user after which further inferences 
can be made. However, pattern recognition relies on the users' epistemological framework, as emphasized by Friis (in terms of a personal horizon) and Rosenberger (in terms of frameworks of interpretation and interpretive skills). In addition to analysing how individual users perceive an image and draw inferences, I am interested in how experts with different disciplinary perspectives together identify relevant patterns in the excess of data in images.

\subsubsection{Alternative theories on imaging}

Authors in sociology provide an analysis of the process in which the evidentiary value of images is established within practices in which the collaborations between professionals are central. For example, Goodwin (1994) argues that "the ability to see relevant entities is not lodged in the individual mind, but instead within a community of competent practitioners," (Goodwin 1994, p. 626). Amann and Knorr Cetina (1988) argue that "just as scientific facts are the end product of complex processes of belief fixation, so is visual 'sense data - just what it is scientists see when they look at the outcome of an experiment - are the end product of socially organized procedures of evidence fixation," (Amann and Knorr Cetina 1988, p. 134). Other authors also take into account the role of specific technologies. Kathrin Friedrich (2010) proposes that the interpretation of medical images depends upon the development of shared 'sight styles' across radiologists in a clinic. Her account draws upon Ludwik Fleck's (1979) notion of 'thought styles' and 'thought collectives,' stressing the social processes and the role of technologies such as software, through which people come to see in the same way. Annamaria Carusi (2011) adds the idea of a visual practice, in which the interpretation of an image takes place in interaction, not only with others or with somebody's background knowledge but also with a physical configuration, usually specific for a certain discipline.

Carusi (2012) argues that, instead of seeing visualisations as cognitive aids to understanding, more attention should be paid to the epistemological role of visualisations. Carusi observed that visualisations in modern, data-intensive and computational science challenges the philosophy of science to reconsider its position on three interrelated key distinctions. First, the qualitative/ quantitative distinction: in the process of formulating scientific claims, scientists continuously move between quantitative parameters and visualisations of these parameters. Both inform each other and with each cycle, the visualisation and the interpretation of the qualitative parameters are modified. Second, the distinction between the subjective and the objective: in science, proof is usually provided through numerical analysis of a pattern and visual/qualitative information is often regarded as more subjective than quantitative information. However, the detection of patterns in large quantities of data is usually performed visually and qualitatively, by people. Finally, 
the distinction between the causal and the non-causal. Objectivity is often aligned with the causal or mechanistic as it seems to exclude subjective bias. The non-causal has been interpreted as relating to meaning, intention or reason. However, this distinction cannot be maintained in modern visualisation techniques, as in the algorithms processing and producing images both causal and intentional factors are involved. Instead of trying to pull the causal and non-causal apart - which is not possible when observing the resulting image or visualisation - epistemological attention should be paid to understanding how they are inter-related.

Bas de Boer et al. (2018) analyse scientific observations through instruments in terms of technological mediations. They argue that it is not only the objective features of an instrument (i.e., the physical interactions between the instrument and the world) that shape a scientific perspective, but also the interpretative framework of the user, while at the same time scientific instruments help interpretative frameworks or scientific theories 'come into being' by shaping the relationship between the scientists and their object of study. According to them "the human parts of a scientific collective must develop a shared epistemic stance with regard to the mediating technology, which allows for multiple coherent interpretations," (De Boer et al. 2018, p. 12). According to De Boer et al., the process of developing this epistemic stance is mediated by the social relations between scientist and the technologies that shape the content of the measurement. Additionally, it is shaped by the (epistemic) actions a collective wish to perform, while at the same time these actions are shaped by the scientific instrument.

Goodwin, Amann and Knorr Cetina, Friedrich, Carusi, and De Boer et al. point out that, in addition to imaging technologies playing an active part shaping the content of the image, and users (and their background knowledge and experience) having a central role in the interpretation of the images, there is a process in which both the content of the image, the working of the technology and the interpretations based on it are established. In the following, I aim to uncover (some of the) epistemological mechanisms underlying this process. I will argue that when imaging technologies and the images they produce are used in an innovative setting the question is not only whether images provide evidence for a certain claim, but also for what claims these images can provide evidence. Researchers are in a process of figuring out what an image represents, and what this means in their specific context, while at the same time tweaking both the technology and the visualisation it brings forth in order to bring out those features that are meaningful and relevant for their practice. 
In medical imaging, this process involves collaborations between professionals with different disciplinary background, i.e., clinicians and engineers, and usually radiologists and radiographers as well. Authors in phenomenology argue that background knowledge and experience play an important role for the interpretation of an image, while sociologists present analyses of how interpretations of images become established in a processes of negotiations between professionals (e.g., Amann \& Knorr Cetina 1988) and argue that groups of professionals develop common ways of looking at visualisations (Goodwin 1994, Friedrich 2010). In my analysis, I aim to uncover the epistemology underlying these processes of interpreting and establishing images by analysing the interdisciplinary collaborative reasoning that leads to epistemic and technological outcomes accepted by these professionals. I will argue that to 'see' something in the images that might serve as evidence for a clinical claim (i.e., evidence on the patient's condition) requires a search process for a pattern that allows making clinically relevant inferences. In this process, different experts have to be involved, because they ask different questions, formulate different hypotheses and interpret the images differently, according to their disciplinary perspective. At the same time, the involvement of expertise from different disciplines introduces challenges associated with interdisciplinary research (also see Chapter 3). Moreover, the concept 'evidence' is in this context a complex and messy one. Images are evidence of an interaction between the imaged object and the imaging technology, which results in a signal that is visualised in an image. The question is, however, how this interaction leads to evidence for a clinical claim. In other words, what patterns will be visible from this interaction (i.e., what the images will represent) and how these patterns relate to the clinical condition of the patient (i.e., how to interpret these patterns). Based on my analysis of the video-recorded deliberative processes, I will show that researchers and clinicians are jointly involved in a search process to find the clinical claims that can be made on the basis of these images. Simultaneously, there is a search process to prove the reliability of these claims. Evidence in this context thus might mean 'that which supports a clinical claim,' as well as 'that which supports that this is evidence for a clinical claim'.

\subsection{Case study}

We have observed and videotaped a mock collaborative task based on real practice of a multidisciplinary team working on the development of a new magnetic resonance imaging (MRI) tool for the diagnosis of airways diseases such as asthma and chronic obstructive pulmonary disease (COPD), called 'hyperpolarized gas MRI' (HP-MRI). For this technique, patients breath in a gaseous contrast agent, either hyperpolarized helium $\left({ }^{3} \mathrm{He}\right)$ or xenon $\left({ }^{129} \mathrm{Xe}\right)$ after which an MRI scan is made. Hyperpolarization refers to a process that 
increases the magnetic susceptibility of the gasses. The scanner is equipped with additional hardware to detect the signal of these gasses, which is different from the signal that is emitted by 'normal' human tissue (which is based on the presence hydrogen protons ${ }^{1} \mathrm{H}$ ) (Wild et al. 2013).

Asthma is one of the most common chronic diseases in children and young adults. It is an allergic inflammatory disorder of the airways that leads to characteristic wheezing episodes and shortness of breath with different severity and frequency, due to narrowing of the airways by constriction of smooth muscle in the bronchi in response to various allergens ('airway hyperresponsiveness'). This narrowing is (partially) reversible with therapy, usually consisting of administration corticosteroids, an anti-inflammatory drug via inhalers, often complemented by bronchodilators for the relief of bronchospasm during acute exacerbations (GINA 2018). COPD is characterized by progressive, largely irreversible airflow obstruction such as emphysema and chronic bronchitis. Symptoms are similar to asthma (i.e., airway hyperresponsiveness and shortness of breath). Therefore, it can be difficult to differentiate between asthma and COPD. One important distinction is reversibility of airways closure: while asthma is reversible, COPD is not. In addition, in COPD airway smooth muscle is not constricted, but the obstruction is the result of damage and loss of alveolar structure of the lungs. In addition, there can be overlap between the two diseases, for example, patients with long-standing asthma that has not been treated sufficiently may develop irreversible airflow reduction. However, it is important to correctly diagnose COPD and asthma, as they require different treatments (Murphy 2011).

The current gold standard to measure lung function is spirometric pulmonary function testing (PFT), generating so-called 'flow-volume loops', in which the measured flow of inhalation and exhalation is plotted against the inhaled and exhaled volume of air. The most important metrics that are acquired with spirometry are forced expiratory volume in one second $\left(\mathrm{FEV}_{1}\right)$, forced vital capacity $(\mathrm{FVC})$, their ratio $\left(\mathrm{FEV}_{1} / \mathrm{FVC}\right)$, and peak expiratory flow (PEF). For asthma, a diagnostic sign is the degree of reversibility of a decreased $\mathrm{FEV}_{1}$ post-bronchodilator (GINA 2018). However, spirometry is limited, as it only provides a global measurement and is relatively insensitive to functional changes in the small airways and gas exchange regions, while the results are very variable as they are dependent on patient effort. The additional value of HP-MRI over spirometry is that it provides regional information on lung ventilation. With HP-MRI, the whole volume of the lungs is scanned and regions that lack ventilation - and thus are not reached by inhaled hyperpolarized gas - can be visualized and quantified. These so-called ventilation defects can be caused by airway obstruction, in the case of asthma, or by damaged lung (alveolar) tissue, as in COPD (Roos et al. 2015). Therefore, it is expected that visualizing 
the extent and location of ventilation defects can contribute to making the distinction between asthma and COPD.

The multidisciplinary team that we have observed consists of a pulmonary consultant clinician ('clinician' - familiar with MRI from his clinical work), a radiologist (specialized in thorax imaging), an MRI physicist ('engineer' - specialized in heart \& lung imaging), and a radiographer. The members of this team had a history of working together, as they had previously collaborated on the introduction of a (relatively) new imaging tool in the practice of diagnosis and evaluation of pulmonary hypertension, called cardiac MRI (CMRI) (see Chapter 5 and Chapter 6 for a discussion of this imaging tool and its introduction into the clinic), and the evaluation of several metrics that can be determined using this technique). Furthermore, all members except for the engineer are involved in weekly multidisciplinary team meetings (MDTs) in which decisions concerning diagnosis and treatment of patients are collectively made (see Chapter 5 and Chapter 6 for a discussion of the role of MDTs in clinical decision-making).

We have videotaped a meeting of the team discussing the HP-MRI scans of two patients that were referred to the researchers by the consultant respiratory clinician (clinician, in short). The recording was transcribed and coded using NVIVO (QRS international Pty Ltd. Version 10, 2012). During the meeting, the images are displayed on a computer screen operated by the radiologist. The clinician is sitting at the desk next to the radiologist, viewing the images. The radiographer and MRI physicist are standing a bit further away from the desk but can see the screen and take part in the discussion. The video recorder was placed so that the screen, the radiologist and the clinician were in view of the camera. See Figure 4.1 for the layout of the room. The cases discussed during the meeting are patients that the clinician had doubts about because their clinical presentation differs from what he had expected based on other measurements and their history. These are the first patients that the clinician has referred for HP-MRI imaging, i.e., the cases for which HP-MRI might serve as input for decisions regarding diagnosis and treatment. The technology is primarily developed by a 'technology development team' that consists of the engineer, the radiographer, the radiologist, and $\mathrm{PhD}$ students and other researchers at the engineer's department. They have developed the hardware and software required to perform the image acquisition and processing, and have tested it on healthy volunteers and asthma patients (patients who were already diagnosed with asthma - to verify whether HP-MRI can represent this pathology). Now, the team is aiming to bridge the gap between the research practice in which these initial technological developments have been realised, and the clinical practice to which the tool should contribute. To do this, the clinician is included in the further development of these images, by referring patients that he thinks 
might benefit from this tool and taking part in meetings like the one in this case study. Immediately after the meeting, with the video recorder still on, we have asked the team to describe their task, which they do (by word of the radiologist) as follows: "multidisciplinary team meeting of a [...] emerging imaging technique, trying to understand... there's some clear clinical aspect to it, but it's trying to understand how much more it could do."

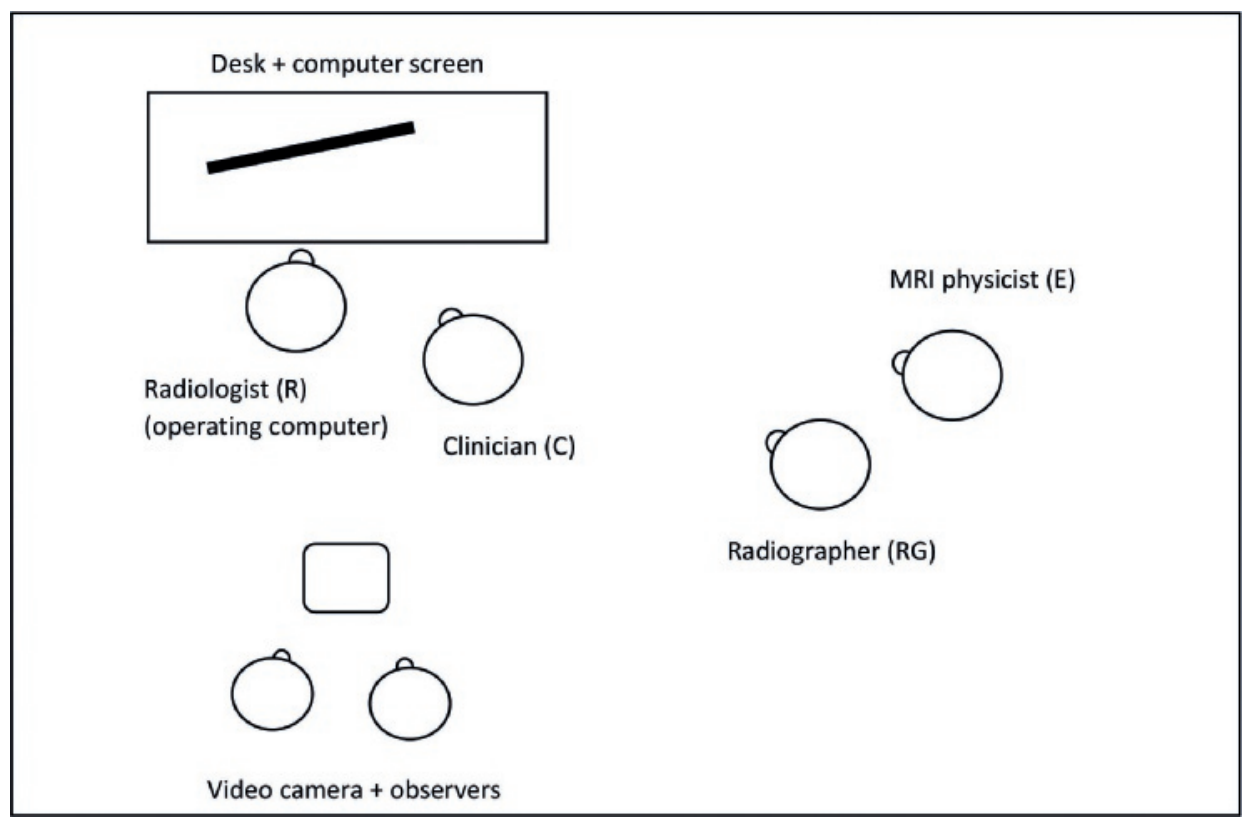

Figure 4.1: room layout during the video-observation

The process by which images of patients are obtained using HP-MRI is as follows. During each MRI scanning session with a patient a range of images is produced at different levels of inhalation (volumes of gas inhaled), namely, at the level (volume) of air or gas that remains in the lungs after normal expiration (i.e., functional residual capacity, FRC) plus one litre of inhaled (hyperpolarized) gas (i.e., FRC + 1), and at the maximum level (volume) of air/gas a person can breathe in (i.e., total lung capacity, TLC). These images were made both before and after the patient used an inhaler with bronchodilators. The radiographer has processed the images so that several metrics (for example, the ventilated volume) are derived and several ways of visualizing are presented. Before the MDT meeting, the radiologist has reviewed the images and metrics and written a report of his findings and interpretations, which the clinician has read. 
Rather than describing the social interactions between these experts in terms of who has which role in the negotiation on what an image means, my analysis of the data aims at making explicit the interdisciplinary collaborative reasoning processes of knowledge formation in interactions between experts with different disciplinary perspectives. The quotes presented throughout the text below are stylized fragments of the original transcript, meaning that repetitions and hesitations are omitted to improve readability and understanding. Occasionally, additional information is filled in between brackets [] in the quote (additions to the quote in 'normal,' interpretations in italics) to bring out more clearly what is being communicated.

\subsection{Searching for evidence}

\subsubsection{Searching for evidence for a clinical claim}

In our video-observation, the clinician starts out by introducing the patient to the rest of the team. He describes how the patient has been having severe attacks of asthma that could not be reduced by medication. Therefore, he has referred the patient for a procedure in which the walls of the bronchi (large airways) are heated, reducing smooth muscle and hence opening up the airways to improve breathing. However, the patient is still having attacks.

\section{Quote 1}

Clinician: "The key question from this was whether there is evidence that despite having [retracted procedure - below indicated as 'the procedure'] he still has reversible airways disease? And if so, how extensive is that? Can you see [on the image] whether it's large airway, where he had the procedure, and is this a procedure failure, or is it small airway, and are we going beyond where his procedure was [supposed to work]"

In Quote 1 the clinician formulates the initial question he expects to be answered by the imaging technology. After a short discussion about the available evidence of the effectiveness of the procedure in the current medical literature, the radiologists summarizes the clinician's initial question, after which the clinician translates his question about what can possibly be observed in these images to what this means for the patient at hand, see Quote 2. 


\section{Quote 2}

Radiologist: "It's an interesting application because that's kind of like separating out problems in the large airways from problems in the small airway."

Clinician: "I'm now trying to work out how aggressive should I be with ongoing immunosuppression." [In case the patient suffers from 'small airway disease' treatment would consist of immunosuppresions, whereas if the patient (still) suffers from large airway disease it would mean that the procedure has not worked and other treatment is needed.]

Quote 3 illustrates that, when the team starts reviewing the images, the radiologist starts out with describing what he sees in the images - segmental defects throughout the lungs - while the engineer (together with the radiographer) draws connections to other cases of asthma patients that he has seen, and the clinician relates it to the expected effect of the procedure with this specific patient. The clinician's expectation is based on his clinical knowledge.

\section{Quote 3}

Radiologist: "This is the baseline FRC + 1 map [FRC = functional residual capacity, the volume of air that remains in the lungs after normal expiration, plus one liter of gas], [...] you can see there's multiple, dominantly segmental defects. And towards the anterior, it's pretty scattered, isn't it?"

Engineer: "that kind of mosaic pattern is quite interesting, we do see that in the asthmatics, do we [name radiographer]"?

Radiographer: "yea."

Engineer: "it's [...] clearly following the segmental structures of the lung"

Radiologist: "yea, sort of segmental, sub-segmental structures. We've got high spatial resolution, you can nicely visualise the edges of the defects."

Clinician: "I mean it seems almost inconceivable he's actually had [the procedure]. Doesn't it? Because, [...] you getting that level of segmental defect." [After the procedure, airway obstructions are expected to have been eliminated, improving ventilation and decreasing defects. Therefore, a smaller amount of segmentation defects are expected.]

The first result of this interdisciplinary collaborative reasoning process is a hypothesis about the patient proposed by the clinician: that the procedure did not have the intended 
effect of relieving airway obstruction. Subsequently, the team decides to test this hypothesis by looking at another set of HP-MRI images of the same patient, which have been made in the same MRI scanning session after using an inhaler to administer a bronchodilator. The team expected that the use of this medication relaxes the airways, leading to a better ventilation of the lungs. Their collaborative interdisciplinary reasoning is analysed as follows: if the ventilation defects observed (i.e., by the radiologist) in the pre-bronchodilator images are caused by spasms in the airways, these bronchi would open up after using a bronchodilator (i.e., predicted by the clinician), opening up the of obstructed ventilation regions. Therefore, fewer ventilation defects would be visible in the post-bronchodilator HP-MRI images, because the hyperpolarized gasses can now also reach those areas that were not-ventilated due to bronchospasm (i.e., concluded by the team). Conversely, if the bronchodilator does not affect the airways, the post-bronchodilator HP-MRI images would likely be similar to the pre-bronchodilator images. ${ }^{35}$ In order to test these hypotheses, in Quote 4, the team reviews the images made after administration of the bronchodilator and compares them to the pre-bronchodilator images. These images are made to assess the reversibility of the ventilation obstruction because this is considered to be a sign of asthma and of the treatability of airway obstruction. Hence, these images do not merely serve as evidence to support the hypothesis about the effect of the bronchodilator, but also for the question concerning the treatability of the airway obstruction.

35 In this video-recording, the team discusses several ways of visualizing data that can be derived from the HP-MRI, which I will not analyse here. 


\section{Quote 4}

Radiologist: "So this is the images now for the post-bronchodilator. [...] You see that a lot of the defects have improved."

[the radiologist and the clinician start by describing the ventilation defects that are visible on both the pre- and post-bronchodilator images, in are thus considered to be 'fixed']

Clinican: "yea, [but] you still kind of got that same ventilation inhomogeneity in the lower right lobe..."

[...]

Radiologist: "and that [inhomogeneity in lower right lobe] looks very similar [to the ventilation defect in the pre-bronchodilator scan], doesn't it? And this [pointing at another ventilation defect] looks fixed as well, up here, very similar pattern. That [ventilation defect], perhaps has filled in [with gas, hence is ventilated] a little." [...]

[after pointing out and describing the fixed ventilation defects, they discuss the reversible defects:]

Radiologist: “And then, you have got some striking differences here, so there's this large defect before, which is largely filled in. And the right, you've completely lost that. The segmental defect down here as well,"

Clinician: "yea"

Radiologist: "this is a kind of large segmental defect."

Clinician: "you'd have thought that if [the procedure] had worked...[there would not have been such large segmental defect that are also reversible.]"

[In sum, the radiologist and the clinician observe more reversible ventilation defects than fixed defects.]

In short, reviewing the post-bronchodilator images, the clinician and radiologist both conclude that many of the defects that were visible in the pre-bronchodilator images have been resolved after using the bronchodilator. Therefore, the radiologist, supported by the interpretation of the clinician (who hypothesized that improvement of defects may occur after administration of the bronchodilator in case the procedure has not worked), concludes that HP-MRI can visualize the reversibility of ventilation defects (rather than these defects being artefacts of the technology), and thus that these images can provide evidence for the occurrence of defects as well as for the reversibility of defects. Simultaneously, based on the radiologist's interpretation, the clinician concludes that the bronchodilator has brought about a (temporary) improvement in this patient, which demonstrates that the defects are reversible. The earlier procedure should have accomplished this, but based on the given interpretations, the clinician concludes that the procedure has not yet worked. In 
sum, in this interdisciplinary collaborative reasoning process, the lines of reasoning by the radiologist and the clinician, who each argue within different disciplinary perspectives, mutually support each other. Based on these findings, in Quote 5, the clinician infers a conclusion on how to continue the treatment of this patient.

\section{Quote 5}

Clinician: "So I think where this is helpful for me, means that I can feel justified in still continuing to be really aggressive with therapy [medication and/or repeating the procedure], because what I'm still seeing is reversible defects...

So if he comes to me and says "I'm having these bad episode" and "I'm still having brittle attacks" you know, [my first] interpretation [of the situation based on the clinical picture] could be that actually he's just lost confidence that [the procedure] has actually worked and things are OK [in this case, the underlying physiological problem of the patient's episode of shortness of breath would have been resolved, but the patient still experiences these episodes because of a lack of confidence in his ability to breath]. [...] Interpretation 2 is, he's in a mess, his [...] [procedure] hasn't done the job. And from the lung function data I think I'm comfortable using interpretation 2 anyway, but these data [the HP-MRI images] reinforce that."

The analysis above aims to illustrate that in order to use images in scientific and clinical reasoning, scientists and clinicians need to figure out what information they can derive from the image before it can be established as evidence for clinical claims concerning diagnosis and treatment. In the case of a new imaging tool (or the new application of an existing tool), it is not evident from the outset what patterns will be visible and how this will relate to the patient's clinical condition. Hence, establishing what evidence an image provides for what claims does not only entail proving that the image represents a certain aspect of patient's condition because this would require that the researchers already know what aspects of the patient's condition will be represented in the images. Instead, my analysis has shown that the radiologist needed to develop his interpretation of the images in a collaborative interdisciplinary reasoning process with the clinician. Therefore, what can be 'seen' in these images has to be figured out in a search process in which both the (future) users (e.g., clinicians) and the developers (e.g., radiologists) are engaged in many (micro-) cycles of asking questions - forming hypothesis - collecting 'evidence' - drawing conclusions.

The discussion sketched in quotes 1- 5 gives an example of how the team explores the information provided by the images and how they get to answer clinical questions on the 
basis of the images. This illustrates that the interpretation of images produced by a new technique is a search process consisting of several steps in which the expertise of each member plays an important role. Crucially, the way in which each expert interprets the images, asks questions for clarification and crafts new hypotheses depends on his or her specific disciplinary perspective, as I have argued in Chapter 3. This claim can be illustrated for the case at hand. Each of the team members has a specific disciplinary perspective that guides what experts 'see' in the images and what research question regarding this imaging technology they ask. In dealing with these research questions and purposes, each expert draws on specific theoretical and practical knowledge: the clinician has knowledge of the specific patient at hand, such as his or her clinical history as well as input from other sources of information, therefore he aims to understand whether and why a specific treatment did or did not work and whether other treatments could be successful, whereas the radiologist is an expert on reading (HP-)MRI images and is therefore interested in whether and how ventilation defects are visualized by the technology. Nevertheless, these experts also have a shared knowledge-base, in this case, about the physiology of asthma, the anatomy of the lung and thorax, and some specificities of treatments. Quotes 1-5 show that the members of the team are, on the one hand, faithful to their own purposes and question, and on the other hand, coordinate relevant knowledge from their own domain of expertise with knowledge from the domains of the other members. I interpret this interdisciplinary medical-technological practice as an intricate process of interdisciplinary collaborative scientific reasoning.

\subsubsection{Philosophical interpretation of scientific reasoning in real practice:}

\section{C.S. Peirce}

Surely, the quotes do not reflect the neat scientific reasoning that we find in textbooks. Instead, we are witnessing a far more complicated argumentation that is being conducted on several layers simultaneously. The layers in reasoning processes that occur in these kinds of professional practices can be interpreted through ideas from Peirce.

Charles S. Peirce (1908) is a pragmatist philosopher who recognized that scientific reasoning in practical situations is less neat and more intricate than philosophers in the logical positivist tradition would like to have it. He gave a reinterpretation of common logical terms, which I will use to interpret the discussion of the four experts and to distinguish several stages. In Peirce's account, scientific reasoning takes place in three stages: abduction, deduction and induction. According to Peirce, although these stages may be expressed as an algorithmic logic, - i.e., as a formal description of how propositions relate to each other - , in actual reasoning contexts it does not tell us how we ought to reason as it is dependent on matters such as background knowledge, purposes and conventions 
of a field, as well as personal elements like creativity or confusion. This is in line with the search process sketched in Quotes 1-5, where an experts' disciplinary perspective shapes epistemic aspects such as the purpose and expectations with which they approach the images, the kinds of questions they ask, the kinds of hypotheses they formulate, and the expert knowledge they introduce in coming to an interpretation of the images.

The first step in Peirce's framework is abduction. In epistemology, the term abduction has come to mean 'inference to the best explanation' - the idea that a hypothesis is accepted because it explains the empirical observations better than competing hypotheses. Peirce, however, defines abduction as something distinct from induction (although he has sometimes called it a 'form of induction' (see Hookway 2012). Whereas in induction a researcher establishes whether it is justified to believe a certain hypothesis, in abduction a researcher establishes the hypothesis he or she is interested in for further interrogation (through deduction and induction). Christopher Hookway (2012) analyses abduction not only as having a different function in Peirce's account but also a different logical form. This logical form involves coming up with a question or 'interrogative' that motivates us to find an answer through induction:

"[A]nd what is such an interrogation but first, a sense that we do not know something; second a desire to know it; and third an effort - implying a willingness to labour - for the sake of seeing how the truth may really be. If that interrogation inspires you, you will be sure to examine the instances; while if it does not, you will pass them by without attention," (Peirce quoted in Hookway 2012, p. 77).

Hookway shows that Peirce suggests that the questions that one comes up with depends on our background knowledge: it must fit in with our presuppositions about a situation, and we must not already know the answer: "our abduction question must be suggested by, and compatible with, the evidence we already possess," (Hookway 2012, p. 79). Hence, our question that is the result of abduction identifies a 'gap' in our knowledge that we wish to fill.

\subsubsection{Abductive reasoning towards a hypothesis in practice}

Peirce thus outlines a logical structure of how researchers come up with a hypothesis that is subsequently investigated and stresses the importance of motivation to investigate something. The elements that he takes to be relevant - i.e., having a gap in knowledge, and a motivation to investigate this gap, as well as already having background knowledge that allows for crafting a hypothesis - are all present in the team's collective reasoning as well. Hence, although the team's reasoning does not follow the neat text-book logic it is not 
unsystematic. It does follow a certain logical structure which was outlined by Peirce. In the following, I will use Peirce's account of logic to analyse the team's search process. In Quote 6 I give an example of an instance of abductive reasoning that leads to a hypothesis that needs to be tested. The clinician introduces the patient to the team members with a presupposition or expectation about the workings of a treatment: "if the procedure had worked, the patient would not have these episodes anymore." He continues with an observation that leads to an interrogative (a question): "what can explain this patient's symptoms?" which expresses that he is worried about his patient and confused about the situation, hence, motivated to find out.

\section{Quote 6}

Clinician: "And he's basically supposed [as a result of the procedure], at this point, therefore, to have much less bronchospasm. Because all the airway's smooth muscle has gone [is expected to have gone after the procedure] in the large airway. But despite the procedure and despite having apparently reversible twitchy airways [as was concluded from viewing the pre-and post-bronchodilator images], he is still having severe attacks and is on the verge of life-threatening asthma. You know, could he have a fatal episode? [because of a lack or wrong treatment]"

[The hypothesis that the clinician wants to be confirmed or rejected is: "The procedure did not work." Reformulated as an 'interrogative' (question): "Did the procedure work?"]

His desire (motivation) to explain his patient's symptoms led the clinician to formulate the diagnostic question with which the encounter in Quotes 1-5 starts, but also to select this patient for (experimental) imaging with HP-MRI as he suspects that it might provide additional information to help this patient. This reasoning implies that the clinician has an expectation (in the sense of 'hope') of what can be seen in the images (i.e., ventilation defects) before actually having seen them. Hence, to come up with the question in the first place, the clinician anticipated how the question was going to be answered and also had some idea of how the technology works, which is based on previous discussions with the engineer and the radiologist. Although the clinician's ideas may need to be adjusted after discussing the first images with the other experts (e.g., the radiologist), these specific background ideas has shaped the way he initially thinks about the phenomenon in question, i.e., about the patient's lungs with or without airway obstruction after the procedure as visualized with HP-MRI. Additionally, this specific background provokes the clinician to ask a clinical question (i.e., did the procedure cure the patient's previous bronchospasms?) 
that he might not have considered if it were not for the prospect of studying this patient on the HP-MRI.

\subsubsection{Deductive reasoning in practice}

Following abduction in which a question or interrogative is set, it must be answered using other types of reasoning. The first step is deduction, which has two parts. In the first, explication, the hypothesis is specified, making it as precise as possible. In the second, demonstration, consequences of the hypothesis are formulated through stringent reasoning. In Chapter 3, I have argued that much of the epistemic work in interdisciplinary collaborations goes into coming up with an interdisciplinary problem definition. The specification of the problem in Peirce's framework can be seen as an instance of this.

Applied to this case, the clinician lays out a clinical problem (Quote 6), but this problem has to be translated into something that can (or is expected to) be visualized using HPMRI, by specifying features in the clinical situation and relating them to features of the HP-MRI images. In this step, the team works out how the images produced by HP-MRI would look if the procedure had worked, thus translating the clinical question into a hypothesis in terms of what can be seen by means of the imaging technology: If the procedure has not worked, this patient will still have airway obstruction, and therefore the HP-MRI images will show ventilation defects. To make these inferences, a great deal of background knowledge - about lung anatomy, physiology, and diseases, and about the procedure of the treatment, as well as about the technology - is required, and throughout the meeting, small adjustments of the problem definition take place to clarify some of it. In the meeting these reasoning steps are not made explicit: the radiologist and engineer quickly turn to describe the observed 'ventilation defects' to study the clinical hypothesis. Regarding the second part of deduction (demonstration), in order to derive how the images might provide an answer to the clinical questions posed by the clinician, an understanding is needed of what features of the disease that can be defined in terms of 'ventilation defects' are relevant (e.g., the shape and location of these defects, and the reversibility of ventilation defects). Summing up, the hypothesis (that resulted from abductive reasoning above) is: "the procedure has not worked." Specifying this hypothesis and demonstrating it (i.e., deriving detectable consequences of the hypothesis) require to reformulate the hypothesis in terms of something that can be detected using HP-MRI, for which this team introduced the concept 'ventilation defects.'

\subsubsection{Inductive reasoning in practice}

Finally, in the last step, induction, these consequences ("if the procedure has not worked, this patient will still have airway obstruction, and therefore the HP-MRI images will 
show ventilation defects") are tested against reality by gathering data. According to Peirce, this starts with classification, in which a connection is made between the hypothesis and the phenomenon by formulating the concepts of the hypothesis in such a way that they correlate to objects in reality. In the case we present here the specified hypothesis and its demonstration (that resulted from deductive reasoning above) need to be tested. To understand the significance of the induction step, it is important to notice that, although it has been established that HP-MRI visualises ventilations defects, members of the team do not know yet exactly what counts as the 'data' that can be gathered with this technology and how this can be quantified (i.e., to indicate the level of ventilation defects) to confirm or refute the hypothesis. For example, in the first viewing of the HP-MRI, the engineer and radiologist make some effort to explicate the shape and location of the ventilation defects on HP-MRI, as can be seen in Quote 3, where they insist that ventilation defects correlate to the segments of the lung and call the defects 'segmental defects.' This draws a connection between the mechanism of the procedure (in which large airways - that ventilate specific segments of the lung - are treated), the conceptualised 'ventilation defects,' and the shape and location of these defects that are seen in the images. However, the fact that the conceptualized ventilation (segmental) defects are actually visible on the images, how they will be visible, and the level and extent of defects that are relevant in this case only becomes apparent in the collaborative process of studying these images. Finding out what counts as 'data' requires input from both domains: knowledge about the effect of the procedure on lung tissue and knowledge of what can be measured or visualized with HP-MRI. Hence, figuring out which image can provide information for which clinical case is actually a crucial part of the search process. For example, the research team in our study realized that an additional scan (a TLC scan, i.e., a total lung capacity scan, which is an image of lungs holding a larger volume of gas inside) could have provided relevant information, which illustrates that it is not obvious from the start where and how to get relevant information (see Quote 7). Part of the search process is, therefore, to determine where to obtain which information, in relation to what information is needed to answer the clinical question.

Finally, classification is followed by probation in which the hypothesis is tested. This last step is actually illustrated in Quotes 1-5, where the members of this team review the preand post-bronchodilator images of the patient's symptoms. 


\section{Quote 7}

Radiographer: "We didn't do TLC [total lung capacity, the maximum volume of air/ gas a person can breathe in] with this guy, we've got $\mathrm{FRC}+1$, pre and post bronchodilator." [the functional residual capacity plus one liter of HP gas, before and after inhalation of bronchodilator]

Clinician: “And why didn't you do TLC?"

Radiographer: "This was the first case we did, and... TLC was actually something we thought of afterwards."

Engineer: "So this is just why you're here, really. What exams would you like to... you know, what is most useful? I think the dynamic information [the effect that breathing in (more) air has on lung tissue] is useful, from what I've seen in the first patients."

\subsection{Searching for clinical claims}

Up to this point, I have analysed the interdisciplinary collaborative reasoning processes of a team of experts using (HP-MRI) images to answer clinical questions. It has been shown that, although the team already discusses HP-MRI images of actual clinical cases, the use of this type of images is not fixed and the team is still trying to figure out which clinically relevant information these images provide, that is, information relevant to decisions concerning diagnosis and treatment of patients. In short, the protocol of image acquisition and processing has not yet been fully established, even as to which (other) types of images are needed (such as a TLC scan, as shown in Quote 7). In other words, besides a process of searching for proof that the images provide evidence for a specific diagnosis, which involves to reliably correlate data of the patient with specific observations in the images, there is a search process to figure out for what clinical claims the images can provide evidence in cases where the patient's clinical presentation is not fully understood. In the latter case, images may provide information that leads to a clinical explanation - i.e., the images may help a clinician to understand the patient's problems, for instance, by testing the hypothesis that the patient's problems are caused by an 'underlying' pathology. An example is the exchange in Quote 8, which took place after the clinician had introduced the second patient. He explains that this is, again, a patient with a complicated set of problems where the patient has breathlessness that could have many causes and is much sicker than what would be expected. The clinician points out that spirometry measurements are not helpful in figuring out what to do with this patient. 


\section{Quote 8}

Clinician: "I'm struggling to work out how to approach his treatment, so evidence that this is asthma with ventilation inhomogeneity, and an understanding of the severity and his treatment response, would just help me navigate in a mine field that's really tricky."

Radiologist: [draws up the images on the screen] "There's a lot of small defects. Predominant small, patchy defects, some segmental."

Clinician: "Could you say this [pattern in the images] is small airways disease rather than large?"

Radiologist: "It suggests that, based on the size and the distribution of the defects. There are some which are a bit larger, like this one here, which could be a larger airway."

Clinician: "Because then it speaks to whether or not it might respond to the procedure. If you do the procedure. Or whether I'll try and use specific inhalers which targeted more toward small airways, for example."

The pattern visible in the second patient is different from the pattern in the first patient. The pattern is the result of the presence of a signal in certain areas. The presence of a signal visualised by the image indicates the presence of gas in the corresponding area of the lung. These areas are regarded as 'ventilated' whereas areas without a signal - i.e., areas that are not reached by the inhaled hyperpolarized gas - are thought not to be ventilated due to obstruction of the airway. This is what they have conceptualized as 'ventilation defects.' In the first patient, the defects were large and appeared to line up with the segmentation of the lungs known from anatomy (see Quote 3). Each of these segments is supplied by its own bronchus and artery. Hence, a segmental ventilation defect is thought to be caused by an obstruction in a larger bronchus, or a large airway. In the second patient, the ventilation defects are small and patchy and therefore thought to be caused by obstructions in smaller airways. Therefore, the team wonders whether the patchy pattern that is visible in the images from the second patient is evidence for small airways disease, whereas the pattern of large, segmental defects visible in the images from the first patient is evidence for large airways disease. This distinction is relevant to the clinician because obstructions in the large airways can be treated by the procedure, whereas obstructions in smaller airways require specific medication administered by inhalers.

In summary, this (first) viewing of HP-MRI images of two patients with ventilation obstructions thus provides clues that these images can be used to support a certain clinical claim, i.e., "the patient suffers from small/ large airway disease." These HP-MRI images 
are from two patients who have two different sets of clinical signs and symptoms that are thought (or assumed) to originate from two different conditions causing airway obstruction - small and large airway disease. However, small and large airway diseases in and by itself are 'unobservable' phenomena. It is a theoretical conceptualisation that helps explain the patient's signs and symptoms and how they relate to other measurements and possible treatments. Small and large airway disease are phenomena that can only be diagnosed indirectly and from the first viewing of the HP-MRI images, it cannot be established how the observed phenomena in the images (described as 'mosaic pattern' or 'patchy pattern') relate to these phenomena. In order to establish this relationship - and with that the usefulness of 'small/large airway disease, as visible on HP-MRI scans,' as evidence for asthma or COPD - it is necessary to find evidence to justify the claim that 'a mosaic pattern on HP-MRI scans is a sign of asthma.' To prove that this pattern is a reliable measure, the team searches for information that is reproducible, i.e., where 'a mosaic pattern on HP-MRI' is similar for similar cases of asthma, to prove that this information can be reliably obtained using HP-MRI.

\subsubsection{Searching for a pattern that is reliable, useful, interpretable and rele- vant}

This process of searching for what the image is an image of is not straightforward. As the radiologist mentions, the patterns in the images suggest that they can provide evidence to distinguish between small or large airways, but he is reluctant to confirm the interpretation proposed by the clinician. In order for this pattern to be reliable, it has to be reproducible. In other words, similar patterns have to be found in similar cases. However, establishing whether cases are similar is challenging, because there is no reliable reference. If the protocol for making the HP-MRI image (e.g., at which volume of inhalation, i.e., TLC or FRC+1, using which hyperpolarized gasses) is standardized and scans are made at fixed inhalation volumes, correlation with the current gold standard, spirometry, is possible. However, as pointed out by the clinician, spirometry is not a perfect measurement. In addition, the information provided by spirometry is limited and does not sufficiently enable clinicians to make those distinctions that are relevant for making diagnosis and treatment decisions (e.g., between small versus large airways defects and the reversibility of defects). Even if spirometry would provide reliable reference points, correlating it with the patterns in HP-MRI is insufficient to reliably confirm the information that the team wants to obtain from the images. That is, with spirometry they cannot confirm that a pattern of large, segmental ventilation defects is evidence for large airway disease whereas a patchy, mosaic pattern is evidence for small airway disease, because small versus large airways cannot be measured with spirometry as it only provides a general measure of the lungs that includes both small and large airways. 


\section{Quote 9}

Clinician: "Of course, the thing is what I'm not looking for from the MRI is just to give me a visual flow volume loop, because I've already got a flow volume loop. I'm looking to try and understand whether the disease is small airways or large, and then maybe start to correlate it with the types of drugs I might use, or to explain why they've got symptoms." [Distinguishing between small and large airways is relevant for the clinician because it leads to different treatment options.]

Engineer: "Which small airways and large airways, yea." [E confirms the question $C$ asks of the images].

Clinician: "And what proportion of any given set of... stuff is reversible, because we see patients with a wide variety of reversible airways disease, and knowing how hard to treat them falls a lot on... how much I can recover." [C asks E to focus his efforts on visualizing the ventilation using HP-MRI in such a way that it is informative regarding the reversibility of the disease, and whether it is small or large airway disease].

In addition to patterns that are reliable and thus reproducible, the team is also looking for patterns that are relevant, or in other words informative to the clinician to differentiate between plausible pathologies that cannot be distinguished using other measurements (see for example Quote 9). This leads to a paradoxical situation: for a new measure to be relevant, it is necessary that it provides information that cannot be obtained otherwise, but for the measure to be reliable it has to be checked against a fixed reference, which cannot be provided by another reliable measure. Hasok Chang (2008) has explored this paradox in Inventing Temperature in which he shows that in order for thermometers to provide a reliable and reproducible scale to measure heat, it is necessary to establish 'fixed points,' at which the temperature is always the same. However, establishing fixed points is a problem in the absence of a reliable measuring device: how can we judge that the fixed points are indeed fixed and not variable? And, more practically, how can the temperature at these fixed points be measured reliably? After consensus was reached that the freezing and boiling of water were the preferred fixed points for thermometry, the scientists that were developing the thermometer had to establish whether water freezing and boiling were indeed fixed. To do this, other instruments, called 'thermoscopes' based on the expansion of fluids were used. Thermoscopes did not provide an absolute measure of temperature according to a scale but were used to determine whether two points had the same temperature. This led many scientists to find that water boiling at different rates had different temperatures. Eventually, scientists started using the steam point of water rather than the boiling point, as they had theoretical reasons to consider it more fixed than the boiling point. Chang introduces the concept of epistemic iteration to characterize the process in which "successive stages of knowledge, each building on the preceding one, are created 
in order to enhance the achievement of certain epistemic goals" (Chang 2008, p. 46). In the case of the thermometer, the thermoscope was associated with the sensation of heat and cold, and the thermoscope was followed by the thermometer based on fixed points. Similarly, to prove whether HP-MRI can provide evidence regarding the reversibility of airway obstruction, and the occurrence of small versus large airway disease, researchers need to find a measurement or metric that can serve as a 'thermoscope' for airway obstruction - in the sense of indicating that two cases are indeed similar without having to provide more detailed information on the condition of the patient -, on which they can build, in subsequent iterative stages of improving the specificity of the measurement. The question is then, how can such a similarity be proven, and what is a relevant property for similarity in these cases: in what way do cases need to be similar. Solving this paradox is an (iterative) process of figuring out how and to what extent the validity of a pattern or measurement can be proven.

Next to reliability and relevancy is the search for a pattern that is useful (also illustrated by Quote 8), in other words, a pattern that not only provides information for making a relevant distinction between pathologies, thus for making a correct diagnosis, but also for making adequate treatment decisions. Ultimately, the aim of a new technology is to improve patient outcomes, that is, an improvement in the patient's condition. This requires a correct diagnosis as well as successful treatment that is also customized to the patient situation. Therefore, the clinician is looking for evidence for a condition that can be treated by a specific treatment (and hypothesizes a link between the patterns in the images and the treatment methods, such as the procedure, in Quote 8).

Additionally, there is a search process to find a visualisation and/or measure that is interpretable by the (future) user. The visualisation must be such that a user (radiologist and/ or clinician) can interpret the image more or less unambiguously for uses in diagnosis and treatment of patients. These last points are a search process that does not take place in one meeting. It is a developmental process (of the imaging technology, the scanning protocol, the image processing, the interpretation of the images in terms of the patient's condition, and the validation) which requires further experimenting with different ways of visualizing the data, presenting them to the user and discussing their interpretations, and using these preliminary findings or interpretations in clinical decisions and subsequently observing the outcome of these decisions. Based on this, small adjustments are made to image acquisition, image processing, and interpretation, in order to bring out those features that are found to be useful and interpretable, as well as relevant and reliable. Hence, the development of the technology is not finished once it first gets tested on patients. The steps that I have distinguished in the search process of the development of an imaging 
technology, in which different experts interact in an interdisciplinary playing field, makes it a process in which the imaging technique and what it represents are established simultaneously, following small, iterative steps of improvement, instead of a linear process in which the technology is first developed and then implemented.

\subsubsection{Searching for a metric}

In addition to providing images that visualize ventilation defects in the lung, the team has also come up with several metrics that can be derived from the images. The purpose of these metrics is to provide an objective, quantitative measure of the condition of the patient, one that does not require recognition or interpretation of a pattern. One of those is the global ventilation point, which is discussed in Quote 10.

\section{Quote 10}

Radiologist: "So the global ventilation point was $92.5 \%$ and that's where [name radiographer] is a [an expert].... do you want to talk quickly about how you actually....." Radiographer: "That's of the total thoracic volume. So we take proton images, in the same breath hold as the helium images, and we'd segment them both and from the ventilation images we get the ventilated volume, and from the proton images we get total lung volume. And then it's just a percentage."

Clinician: "Because that looks worse than $92 \%$, doesn't it?"

Engineer: "In that slice, yea, you've got to bear in mind the other slices are better ventilated. So you're making a global metric."

Clinician: “And it's not quality of ventilation it's so much as whether ventilation is present or absent. [Engineer and Radiologist simultaneously: "binary"]. So actually, these areas which are very poorly ventilated aren't contributing to that $92 \%$ ? They're ventilated."

Radiographer: "They're passed as ventilated."

Clinician: "So they're part of that 92?"

Radiographer: "Yes, exactly."

In the fragment, the radiographer introduces the 'global ventilation point' as a metric. As illustrated in the fragment, the metric is not intuitively obvious to the clinician. Firstly, he needs some additional information on how the metric is calculated in order to gain an understanding of what is included in the metric, i.e., the whole lung volume rather than one slice and each area that is ventilated is counted 'in' regardless of the strength of the signal. From this, the clinician starts forming an idea of what the metric tells him. However, what it seems to tell is different from what he expected from viewing the 
images. For, $92 \%$ is quite a high percentage whereas from the images - in which a large number of ventilation defects are visible - he expects a much lower number. This mismatch between the clinician's expectation and the metric results in a discussion about the interpretation of the metric. The radiologist explains that he can provide a more nuanced presentation of the metric in the written report. However, this still leaves the clinician in doubt about how the metric relates to his patient, as he wants to attribute meaning to the numbers. This requires not only analysing more patients so that the radiologist will develop a more detailed sense of the correlation of the number to the severity of the case, but also validating the number against the general picture the clinician has formed of the patient, based on many other sources of information (Chapter 1 and 2) and the eventual outcome after treatment. The 'global ventilation point' (in Quote 10) might be a very precise and reproducible metric for ventilation as measured by HP-MRI, which is what the engineer strives to accomplish, but for the clinician the metric also needs to be informative regarding the patient's condition, and it needs to add to as well as fit in with other information that informs the clinical picture. In other words, information that the imaging technology provides does not only have to be reliable (largely the domain of the engineer and radiographer, and to a lesser degree the radiologist), but it also needs to be relevant, useful and interpretable (largely the domain of the clinician and radiologist).

Arriving at metrics that meet these criteria requires a long iterative process, which progresses in small steps through an ongoing conversation between all members of the team who exchange interpretations and carefully coordinate knowledge from different knowledge bases. The video-recorded meeting was only at the beginning of this process. Throughout it, questions are answered and confusions are cleared up, but new questions were raised that will be clarified in small sub-studies. For example, in Quote 11, the clinician expresses his struggle with the metric and the radiologist suggests ways to improve the interpretability of the metric and to link it to the patient outcome (referred to as 'follow up'), supplemented by the engineer's suggestion to look at earlier images in order to develop a scoring that is helpful for the clinician. 


\section{Quote 11}

Clinician: "Because, you see, I was quite struggling with this one, it looks really patchy, but then apparently it's well ventilated. And, actually, I totally get how you're making your number. But what your number doesn't give me is the quality of the ventilation."

Radiologist: "Yea, no, I totally appreciate that. [...] And I think that the variation is important as well. But, we'll do a description as well. And, perhaps [score it as] mild, moderate and severe, to link it to the follow up, so you get an impression of... sometimes it's only in the texts of the report to provide that information."

[...]

Engineer: "We could go back retrospectively and look at them [HP-MRI images that were made earlier] qualitatively. We have got a body of images from last year." Radiologist: "Yea and then to do some, some scoring for comparison."

Clinician: "But I think knowing the limits, or knowing how you're going to report them, it helps me work out who I can send you that you find useful for baselining it. Who I would find useful for baselining, toward getting clinical info."

In addition, viewing the images while at the same time getting a sense of how the radiologist will score them (e.g., as mild, moderate or severe) also helps shape the clinician's idea of the kind of patients that he thinks the technology can provide useful information for, in other words, what clinical question can this technology help answer by providing what relevant evidence.

Similar events occur throughout the whole meeting: confusions, uncertainties or questions that arise during the exchange lead to new ideas for research or for possible clinically relevant interpretations, for example in Quote 12, when the engineer realizes that one of the things that the clinician is interested in is figuring out whether a patient suffers from large or small airway disease, and that this might be visualised by indicating whether ventilation defects follow the segmental structures of the lung. Therefore, he suggests a way to prove whether defects line up with the lung segments or not. This, in turn, leads the clinician to suggest how this information may be relevant for making treatment decisions as well. 


\section{Quote 12}

Engineer: "But what would be quite interesting for us to do in the future is, put these on top of the CT [which provides a visualisation of the lung tissue, including the segments of the lung], and have the lobes and segments, segmented, than you can start to see. Which segments and which feeding bronchus is actually, that starts, you know, has each bronchus been, bronchiole been treated the same. And do a segmental analysis."

Radiologist: "That would be good."

Clinician: "You'd even consider whether you need to focus your [procedure] efforts more on one part of the lung [instead of applying the procedure to the whole lung]."

\subsubsection{Searching for the best representation of the data}

Unlike photographs or X-ray, MRI images are not a map of interactions between a certain waveform and the human body. Rather, it is a reconstruction of a complex procedure based on interactions between protons, or in case of HP-MRI, helium or xenon atoms, at a quantum mechanical level, producing a frequency signal that is, at first, collected in a virtual space called ' $\mathrm{k}$-space' before a spatial distribution can be constructed. Therefore, an important area in which adjustments can be made to bring out relevant features and facilitate useful interpretations is in how the data is presented. In the previous paragraphs, we have shown that the way images are presented should accord with how the user (the clinician) will use the image for deriving conclusions in diagnosis and treatment, and what kind of images they are familiar with. Kelly Joyce (2006) reconstructs how the representation of the MRI data is transformed after introduction to clinical practice. Initially, MRI scanners produced full-colour images and an array of numbers was printed alongside the anatomic pictures. Driven by radiologist's preference for viewing this information in the form of images (rather than as arrays of numbers) and their familiarity with representations produced by other imaging technologies (e.g., X-ray and computed tomography $(\mathrm{CT})$ ), these were later changed to into grey-scale images. In her words "there is nothing natural or inevitable about how MRI is presently designed or interpreted," (Joyce 2006, p.1). Megan Delehanty (2010) sketches a similar process for CT and shows that the data display format has a large impact on the ability to make certain discriminations. Any cognitive advantage that images might have for a specific user such as the clinician, over other forms of data representation (e.g., numerical), is highly dependent on the chosen display format (e.g. full colour or grey-scale).

In short, there is a considerable amount of leeway to shape how the data recorded by MRI is visualized, and the choices that are made have an impact on the use and interpretation of 
the images. The search process therefore also involves figuring out what way of presenting the data may lead to representations that are interpretable to the clinician. This is a process that takes multiple iterations and is not resolved in one meeting. Over the course of the exchange, the research team discusses several ways of presenting the data and the engineer also provides several other suggestions, as illustrated in Quotes 13 and 14.

\section{Quote 13}

Engineer: "That's where I think the histograms of the ventilation images come in. What would be nice, down in the bottom corner is a histogram distribution of that image. So if you could see the histogram of the distribution of the ventilation, alongside the slice, and something like the full width half max. Or the kurtosis of that histogram and then you've got, you've got two numbers, which...”

\section{Quote 14}

Engineer: "So the level of greenness and redness in there is the factor, you can't really see it on this colour scan. [...] It looks green or red, but the intensity here is response to the magnitude of the change in ventilation as well... before and after. So there are visual ways of looking at that. These histograms, and we can explain that in a bit more depth, because this is something I think we should [lean] more towards, like every pixel is a measurement in its own right and it's not just a.... ventilated/ not ventilated.

Clinician: "It's trying to show me how I should think about these numbers? Because that's key thing."

Radiologist: "It gives you an overall positive and negative change."

Figuring out what can be seen in the data and how to present it in such a way that it is interpretable takes time, experimentation and close collaboration between different experts. As illustrated above, what is seen in images is very dependent on the way the images are presented. However, as pointed out by phenomenologists, what is seen in the images, the gestalt, is also shaped by the observer's background knowledge and experience (Friis 2015; Rosenberger 2009). What phenomenologists do not take into account is that what is seen is also established in collaborations like the one I have sketched out in this chapter. I have characterized the development and use of new imaging technologies as a search process for patterns that are reliable, relevant, useful and interpretable. This search process involves figuring out for what clinical claims the images can provide evidence, how proof for the 
validity of this evidence can be obtained, and how to present the data in such a way that relevant features can easily be identified. This is not a linear and straightforward process but takes many iterations in which confusions and questions lead to new ideas to tweak the technology and its interpretations that are followed up in subsequent meetings and tested against the clinical practice by relating them to clinical outcome.

\subsection{Conclusion}

The success of developing a new imaging tool for clinical practice hinges on the reliability, relevance, usefulness and interpretability of the tools for the intended uses (e.g., diagnosis). Therefore, a close collaboration of developers with future users, i.e., the clinicians, is imperative for this type of research practices. In Chapter 3 I have argued that different experts bring different disciplinary perspectives to the table and that these perspectives shape how they deal with a target system, including the different epistemic values with which they evaluate the result. Here, I have zoomed in on the interactions between different experts, firstly to illustrate how interdisciplinary collaborative reasoning works in practice. I show that from their different perspectives experts ask different questions and provide different, but equally valuable insights on the developing technology. Their contributions feed to each other: suggestions and questions from the clinicians help the engineer to articulate follow-up research, whereas the interpretations by the radiologists help the clinician to better understand his patient's condition. In this way, medical imaging development is not a straightfoward linear process of developing a technology and then introducing it into practice but is an iterative one where new interpretations lead to new research questions and directions to further tweak and develop the tool.

In this chapter, I show that establishing what is 'seen' and what the image can 'tell' about the patient's condition is a search process in which the user(s) search for something that is reliable and relevant for the specific context. Reliable, in this search process means that measures are obtained in a technologically sound manner and that measures are a good representation of the pathophysiological process. Establishing the reliability of images is usually seen as belonging to the domain of engineers, who do this prior to discussing the images with clinicians. Relevant means that it provides information about the patient that has an impact on diagnosis and treatment decisions. This task is usually attributed to the clinician, or at least to a later stage in the development process after the reliability of a measure has been established. However, in this chapter, I showed that it is not evident from the outset exactly what pathophysiological processes will be visualized by the images and 
how they will appear on the images (although all team members have expectations and presuppositions about this). A good example of this can be found in Quote 8, where the clinician, the radiologist and the engineer together reflect on whether a certain pattern (i.e., patchy versus segmental) represents a certain pathophysiology (i.e., small versus large airway disease). It is by finding relations between the patient's history, signs and symptoms (provided by the clinician), and the patterns in the images, that the team develops an idea of what these images can represent. Deciding which of these relations are worth further investigation is directed by the expected relevance for the clinicians' practice. This then leads to further investigations. For example, the discussion in Quote 9 provided direction for subsequent research to establish whether the images reliably represent these pathologies. This is what happens in Quote 12, where the engineer comes up with a way to combine the new images with familiar ones to check whether the pattern aligns with known anatomy. This step in the development process is the outcome of a shared search process. In other words, reliability and relevance are intertwined and need to be established side by side, not one after the other.

A central place for this development process is the multidisciplinary team (MDT) meeting such as the meeting that I have analysed in this chapter. In these meetings, team members coordinate and adapt their contributions to each other to collaboratively establish what they 'see' in these pictures and what that means for clinical practice. For example in Quote 4 the clinician and the radiologist describe what they see in the image, and check this with each other ('and that looks very similar, doesn't it?'). In a similar way, they coordinate and adapt their epistemic aims and research questions, as I show in Quote 7, to establish together what is worth looking at in these images and what about them is worth further investigation.

That these interdisciplinary collaborative reasoning processes of coordination, adaptation and searching are imperative for the development of a useful imaging tool is also evident in Quote 10. The engineer and radiographer have come up with a metric that should provide an objective measure for the severity of lung obstruction. They have worked on a metric that represents the HP-MRI signal throughout the lung in one number as well as possible. However, this metric is not intuitively conceivable to the clinician, as he seems to be seeing something else when he looks at the images. In Chapter 3, I have mentioned that there is a difference between the preciseness and the accuracy of a model. A similar type of distinction seems to be in play here as well: the metric might very precisely reflect the signal in the sense that each pixel where a signal is detected is counted and each pixel with no signal is not, but it might not be an accurate metric in the sense that it does not represent the severity of the ventilation obstruction. Without the involvement of a clini- 
cian, the researchers with a technological focus (i.e., the radiographer and the engineer) run into the risk of overly focusing on the preciseness of the metric, as this is what they can control by improving the technology, whereas the clinician is more interested in an accurate metric. Conversely, when a clinician reviews the metric without receiving direct feedback from the technological team members, he might dismiss it because his interpretation of the number does not fit in with his interpretation with the images. In other words, to establish the meaning of the metrics for clinical practice, the team members need to coordinate their interpretations of the image in relation to other interpretations of other measurements.

Finally, I want to point out two further aspects of the interactions that I have presented here. The interpretation of the images, the empirical goals, and research questions of team members converge over time, in interdisciplinary collaborative reasoning processes that focus on small details concerning the overall development of MRI technology. It is through reviewing the details of the images and the patient's conditions that a team ultimately establishes how and in what way the imaging tool can have an impact on clinical practice and in what direction the development of the imaging tool should proceed. See for example Quote 11, where the mismatch between the calculated number (the metric) and the observed pattern leads to the decision to review a larger set of images in order to obtain a more nuanced understanding of the metric. Secondly, the task in which the team is engaged in the case that I present in this chapter can be considered interdisciplinary in much the same way as I have presented in Chapter 3. Similar to that case, team members are not concerned with developing a new theory, and hence with integrating theories or concepts from their respective fields. Rather, theory plays an instrumental role in better understanding what they see in the images and how that relates to the patient's condition. What is ultimately the goal of these collaborations is to develop a technologically sound tool that has an impact on clinical decision-making concerning diagnosis and treatment of individual patients. 


\section{References}

Amann, K. and Cetina, K. Knorr (1988), 'The Fixation of (Visual) Evidence', Human Studies, 11 (2/3), 133-69

Butler, D. (2008), 'Translational research: Crossing the valley of death', Nature 453, 840-42 DOI: 10.1038/453840a

Carusi, A. (2011), 'Computational Biology and the Limits of Shared Vision', Perspectives on Science, 19 (3), 300-36 DOI: 10.1162/POSC_a_00044.

Carusi, A. (2012), 'Making the Visual Visible in Philosophy of Science', Spontaneous Generations, 6 (1), 106-14 DOI: $10.4245 /$ sponge.v6i1.16141.

Chang, H. (2008), Inventing Temperature (Oxford: Oxford Studies in the Philosophy of Science) ISBN: 9780195337389.

de Boer, B., Te Molder, H., and Verbeek, P.P. (2018), 'The Perspective of the Instruments: Mediating Collectivity', Foundations of Science, DOI: 10.1007/s10699-018-9545-3.

Delehanty, Megan (2010), 'Why Images?', Medicine Studies, 2 (3), 161-73 DOI: 10.1007/s12376-010-0052-2.

Fleck, L. (1979), Genesis and development of a scientific fact (Chicago: University of Chicago Press) ISBN: 9780226253251.

Friedrich, K. (2010), “Sehkollektiv’: Sight Styles in Diagnostic Computed Tomography', Medicine Studies, 2 (3), 185-95 DOI: 10.1007/s12376-010-0050-4.

Friis, J.K.B.O. (2015), 'Gestalt descriptions embodiments and medical image interpretation', Ai \& Society, DOI: 10.1007/s00146-015-0615-6.

Giere, R.N. (2006), Scientific perspectivism (University of Chicago Press) ISBN: 9780226292137.

GINA, global initiative for asthma (2018), 'Online appendix to global strategy for asthma management and prevention', <https://ginasthma.org/2017-online-appendix-global-strategy-for-asthma-management-andprevention/>, accessed 30-08-2018.

Goodwin, C. (1994), 'Professional Vision', American Anthropologist, 96 (3), 606-33

Hacking, I (1981), 'Do we see through a microscope?', in P.M. Churchland and C.A. Hooker (eds.), Images of Science (62; Chicago: The University of Chicago Press) ISBN: 9780226106540

Hookway, C. (2012), 'Interrogatives and Uncontrollable Abductions', The pragmatic maxim: essays on Peirce and pragmatism. (Oxford: Oxford University Press), 77-82 ISBN: 9780199588381 DOI: 10.1093/ acprof:oso/9780199588381.001.0001

Ihde, D. (1986), Experimental phenomenology: An introduction. (Albany: State University of New York Press) ISBN: 0-88706-199-0.

Joyce, K. (2005), 'Appealing Images: Magnetic Resonance Imaging and the Production of Authoritative Knowledge', Social Studies of Science, 35 (3), 437-62 DOI: 10.1177/0306312705050180.

Joyce, K. (2006), 'From numbers to pictures: The development of magnetic resonance imaging and the visual turn in medicine', Science as Culture, 15 (1), 1-22 DOI: 10.1080/09505430600639322.

Kulvicki, J. (2010), 'Knowing with Images: Medium and Message', Philosophy of Science, 77 (2), 295-313 DOI: $10.1086 / 651321$.

Murphy, A. (2018), 'Knowing the differences between COPD and asthma is vital to good practice. The pharmaceutical journal.', <https://www.pharmaceutical-journal.com/learning/learning-article/knowingthe-differences-between-copd-and-asthma-is-vital-to-good-practice/11085597.article?firstPass=false $>$, accessed 30-08-2018.

Peirce, C.S. (2018), 'A Neglected argument for the reality of God', <http://en.wikisource.org/wiki/A_Neglected_ Argument_for_the_Reality_of_God>, accessed 24-0.

Perini, L. (2012), 'Image Interpretation: Bridging the Gap from Mechanically Produced Image to Representation', International Studies in the Philosophy of Science, 26 (2), 153-70 DOI: 10.1080/02698595.2012.703478. 
Roos, J. E. et al. (2015), 'Hyperpolarized Gas MRI: Technique and Applications', Magnetic resonance imaging clinics of North America, 23 (2), 217-29 DOI: 10.1016/j.mric.2015.01.003.

Rosenberger, R. (2009), 'A Case Study in the Applied Philosophy of Imaging: The Synaptic Vesicle Debate', Science, Technology \& Human Values, 36 (1), 6-32 DOI: 10.1177/0162243909337117.

Suárez, M. (2004), 'An Inferential Conception of Scientific Representation', Philosophy of Science, 71 (5), $767-79$ DOI: $10.1086 / 421415$.

van der Laan, A. L. and Boenink, M. (2015), 'Beyond Bench and Bedside: Disentangling the Concept of Translational Research', Health Care Analysis, 23 (1), 32-49 DOI: 10.1007/s10728-012-0236-x.

Wild, J.M. et al. (2013), 'Simultaneous Imaging of Lung Structure and Function with Triple-Nuclear Hybrid MR Imaging', Radiology, 267 (1), 251-55 DOI: 10.1148/radiol.12121153. 


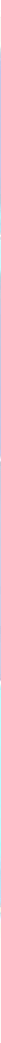




\section{A social-technological epistemology of clinical decision- making as mediated by imaging}

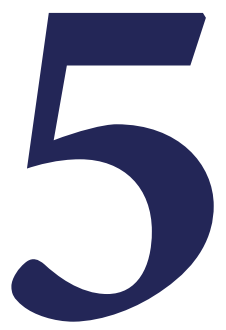

This chapter is published as: S. van Baalen, A. Carusi, I. Sabroe, \& D. G. Kiely (2016). A social-technological epistemology of clinical decision-making as mediated by imaging. J Eval Clin Pract, 23(5), 949-958. DOI: 10.1111/jep.12637

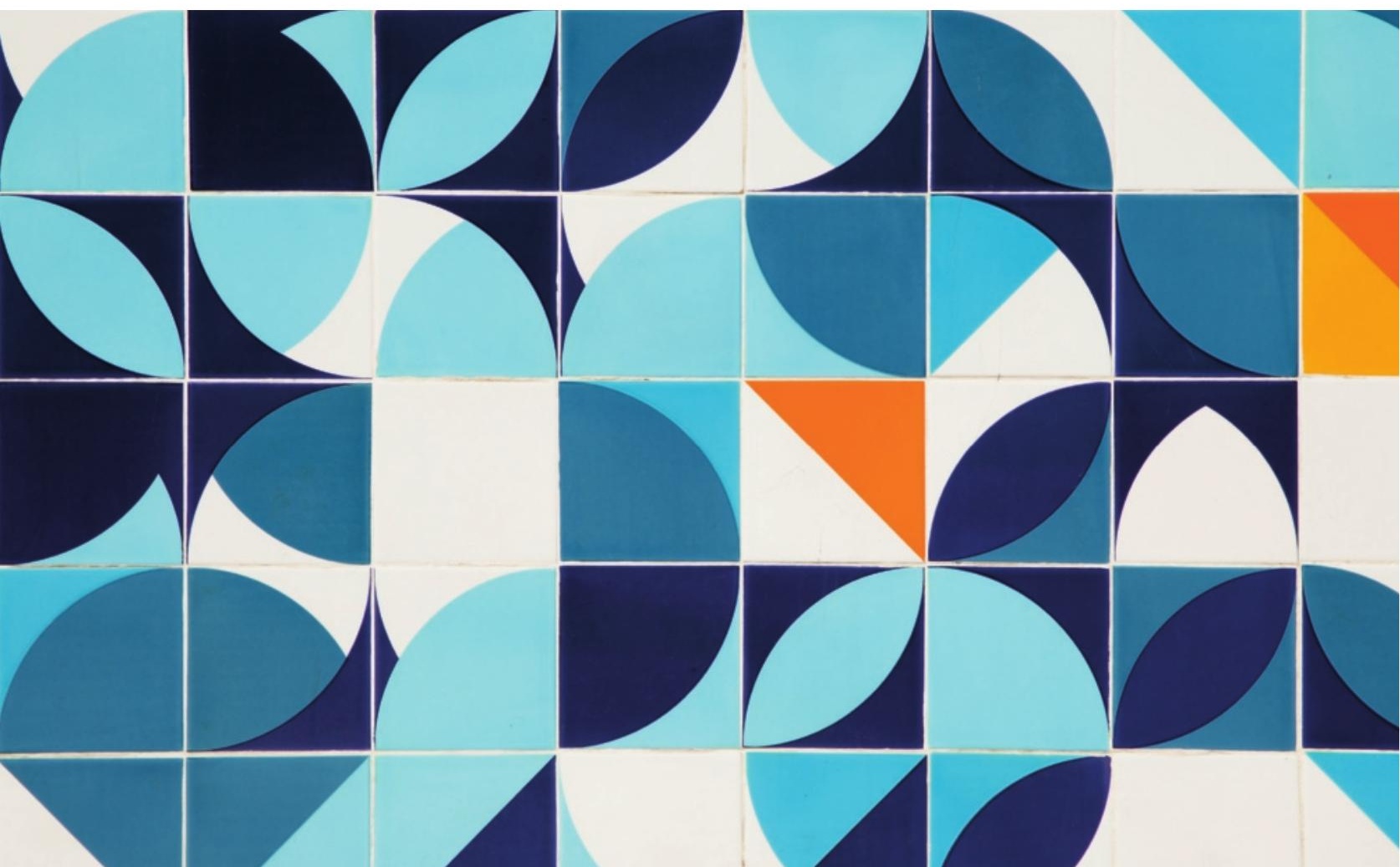




\begin{abstract}
In recent years there has been growing attention to the epistemology of clinical decision-making, but most studies have taken the individual physicians as the central object of analysis. In this paper we argue that knowing in current medical practice has an inherently social character, and that imaging plays a mediating role in these practices. We have analysed clinical decision-making within a medical expert team involved in diagnosis and treatment of patients with pulmonary hypertension $(\mathrm{PH})$, a rare disease requiring multidisciplinary team involvement in diagnosis and management. Within our field study, we conducted observations, interviews, video tasks and a panel discussion. Decision-making in the $\mathrm{PH}$ clinic involves combining evidence from heterogeneous sources into a cohesive framing of a patient, in which interpretations of the different sources can be made consistent with each other. Because pieces of evidence are generated by people with different expertises and interpretation and adjustments take place in interaction between different experts, we argue that this process is socially distributed. Multidisciplinary team meetings are an important place where information is shared, discussed, interpreted and adjusted, allowing to develop a collective way of seeing and a shared language. We demonstrate this with an example of image processing in the $\mathrm{PH}$ service, an instance in which knowledge is distributed over multiple people who play a crucial role in generating an evaluation of right heart function. Finally, we argue that images fulfil a mediating role in distributed knowing in three ways: first, as enablers or tools in acquiring information; second, as communication facilitators; and third, as pervasively framing the epistemic domain. With this study of clinical decision-making in diagnosis and treatment of $\mathrm{PH}$, we have shown that clinical decision-making is highly social and mediated by technologies. The epistemology of clinical decision-making should take social and technological medication into account.
\end{abstract}




\subsection{Introduction}

In recent years there has been a growing attention to the epistemology of clinical decision-making (Braude 2009; Kennedy 2013; Khushf 1999; Loughlin 2008, 2009; Montgomery 2006; Solomon 2008; Tonelli 2006, 2010). In this paper we argue that knowing in current medical practice has an inherently social character and that technologies such as imaging play a crucial and mediating role in these practices. The epistemology of clinical decision-making should take the social and technological mediation of clinical decision-making into account.

\subsubsection{Teamwork in clinical practice}

That teamwork is a crucial aspect in medical practice has been recognized in sociological studies of medical practice. For example, several studies (Cicourel 1990; Hindmarsh and Pilnick 2002; Koschmann et al. 2007; Koschmann et al. 2011) draw attention to teamwork in the operating theatre, while others describe teamwork in clinical decision-making. Aaron Cicourel (1990) characterises the clinical diagnostic process as socially distributed cognition, which "refers to the fact that participants in collaborative work relationships are likely to vary in knowledge they possess," (Cicourel 1990). Within this process, "physicians typically assess the adequacy of medical information on the basis of the perceived credibility of the source, whether the source is the patient or another physician" (ibid, p. 222). Through discourse, physicians assess the area and level of expertise of their coworkers and the reliability of their patient's account of his or her illness to be able to evaluate the robustness of the information provided.

Per Maseide (2006) also characterises medical decision-making as socially distributed cognition: "A ward conference, with a number of individual members with different qualifications, functions, responsibilities, skills and experiences, has knowledge and memory structures, procedures of reasoning and practical qualifications that are socially distributed and differ from the cognitive capabilities of the individual participants," (Maseide 2006, p. 44). He identifies four forms of evidence that "influence and regulate the judgments and decisions of medical practitioners" (ibid, p. 44): scientific evidence, evidence from personal experience, evidence as medical representation artefacts, such as images and pathology results and 'practical evidence,' which is, according to Maseide, closely integrated with forms two and three. This last form of evidence is cooperatively and collaboratively constructed: "practical medical evidence is generated, developed and made useful locally by medical practitioners," (ibid, p. 44). There are several seminal studies of the social aspects of decision-making in the tradition of distributed cognition. Trevor Cohen (2006), for example, considers decision-making in the context of a psychiatric ward, drawing upon a 
theoretical framework initially developed by Hutchins (1995). This framework considers cognition to be distributed across individuals and artefacts, combining internal representations (in the minds of individuals) and external representations (in physical media, such as shared whiteboards). The account proposed here covers similar terrain, in that it stresses the social - or distributed - nature of knowledge processes, and the role of media, such as images. However, as will be discussed later, there are important differences between distributed cognition theory and our own.

This paper's main concern is with epistemology, that is, it aims to shed light on the epistemology of clinical decision-making, and also to contribute to the development of philosophical epistemologies able to cope with these kinds of contexts. The paper argues that the social nature of clinical decision-making is an ineliminable aspect of its epistemology. By this we mean that an individualist epistemology, based on a traditional analysis of knowledge in terms of individual knowers, is not adequate as a basis for an account of knowledge in clinical contexts and that sociability is a necessary aspect of the epistemology of clinical decision-making. The social character of knowledge in other spheres has been recognized by many philosophers, an early example in the turn to social knowing in scientific contexts being John Hardwig (1991) who writes: "Knowing, then, is often not a privileged psychological state. If it is a privileged state at all, it is a privileged social state. So, we need an epistemological analysis of the social structure that makes the members of some teams knowers while the members of others are not," (Hardwig 1991, p. 697). The social structures of knowledge are increasingly acknowledged in philosophical studies of scientific practice (Andersen and Wagenknecht 2013; Andersen 2013; Hardwig 1985, 1991; Wagenknecht 2015), but this has not received the same degree of attention in philosophical accounts of clinical decision-making. For example, Kathryn Montgomery's (2006) detailed analysis of how physicians deal with uncertainty and incomplete information in clinical decision-making focuses on the individual doctor in clinical-patient interactions. Montgomery describes medical case conferences in one of her chapters, foregrounding the establishment of authority and hierarchy, as one aspect of the sociability of knowledge practices (Montgomery 2006). Cunningham (2014) shows the extent to which the sociability of clinical decision-making is increasingly acknowledged as a challenge in philosophy of clinical decision-making. He uses a distributed cognition theoretical framework as the core of his normative account of clinical decision-making (Cunningham 2013). While overlapping in our concerns, the socio-technological epistemology we propose differs in its theoretical orientation, as discussed in the concluding section of the paper. Finally, we note that the position we advocate does not imply a 'collective knower' over and above group members, but we will not enter into this debate here. Rather, our interest is in showing that the epistemology of decisions regarding diagnosis and treatment is not fully accounted for 
by appeal to individual knowers, and does not do justice to the complex coordination of team members with different expertises, where social interactions play a pivotal role. The social epistemology we propose is better able to develop an understanding of diagnosis and management decisions made in clinical teams that are becoming increasingly complex. Rapid changes in availability and quality of imaging, development of new and expensive drugs, and an increasing realisation of the need to place medicine in a social construct for patient benefit have driven the multidisciplinary team (MDT) to come to the fore as a central place for shared decision making.

The essential role of technologies is also not normally included in accounts of knowledge in clinical decision-making. Technologies are normally relegated to vehicles for evidence, but we argue that technologies, and evidence and knowledge claims made on their basis, coevolve with each other and play an essential role in mediating the social knowledge of clinical contexts. In this regard, images are most often analysed in terms of their ability to provide evidence for (scientific) claims. For example, Laura Perini (2012) analyses how mechanically produced images are structurally related to the shape of the specimen being imaged by being sensitive to certain aspects of a specimen and indifferent to others (Perini 2012). However, most medical imaging techniques are not purely mechanically produced, but have a substantive informational component too. For example, Carusi (2012) argues "embodied in the algorithm for image processing, there is a hybridity of causal factors (the way in which the algorithm organizes shapes and contours in the image) and intentional/ informational factors. The resultant images that are viewed for further interpretations are a hybrid of causal and non-causal factors" (Carusi 2012, p. 111). In other words, the image is not the result of a chain of causal factors, but of causal factors combined with factors like processing algorithms, that are programmed with an intention to filter, simplify or interpolate data. Hence, medical images cannot be simply regarded as "vehicles for seeing-in" the body and image technologies as "visual prosthetics" that provides direct access to the inside of patients (Semczyszyn 2010).

In addition, several authors have argued that the process of image analysis by radiologists or clinicians can be understood as a hermeneutic system. According to Jan Kyrre Berg Friis (2015) the interpretation of images takes place preconsciously. He characterises image interpretation in terms of the hermeneutic circle, in which the mind moves from parts to whole and back to make sense of an image. Friis invokes the concept of gestalt to analyse the interpretation of medical images. In his words, gestalts are "something that stand out against a background and enables us to identify patterns," (Friis 2015, p. 2) and that cannot be understood as a feature of the image itself, but of the image in interaction with the perceiver. The perceiver is in turn shaped by his or her background: Friis argues that 
visual perception is an embodied skill that is shaped by biology, society, experiences and training that together make up that a personal 'horizon' meaning that visual perception is variable from person to person. Therefore, two radiologists may interpret the same image differently, because of variability in their horizon. Robert Rosenberger (2009) argues that most images are multistable, meaning that they can be interpreted differently by applying a different hermeneutic strategy, informed by "theoretical commitments, explanations of the structures within the image's content, and relations to the imaging technologies," (Rosenberger 2009, p.15). He applies this theory to an ongoing debate in neurology, where the two opposing parties interpret the same images differently. Questioning each party's hermeneutic strategy, Rosenberger argues, can suggest further trajectories for research by asking for a more detailed account of the morphologies present in the image according to rivalling theories. These authors give an account of how image and interpreter interact, but omit an understanding of how this interaction shapes the social distribution of knowing and mediates the interaction between different members of clinical groupings. This is what we focus on in this paper. In her article on computed tomography (CT) images as diagnostic tools, Kathrin Friedrich (2010) proposes that the interpretation of these images depends upon the development of shared 'sight styles' across radiologists in a clinic (Friedrich 2010). Her account draws upon Ludwik Fleck's notion of 'thought styles' and 'thought collectives,' stressing the social processes and the role of technologies such as software, through which people come to see in the same way (Fleck 1979, 1986e [1947]). As will become apparent, the account we propose here is similarly oriented towards the development of shared modes of seeing.

In summary, we will argue that rather than focusing on the individual clinician's reasoning and knowledge, it is more fruitful to think of clinical decision-making as a form of social knowing, in which technologies play a key mediating role. In such a system, decision-making cannot be performed by any one individual, but is instead performed by an assemblage of people and instruments in coordinated actions. This paper examines clinical decision-making through a detailed study of image-assisted diagnosis and treatment of a pulmonary disease. The study shows the knowledge processes involved among the different epistemic agents with different expertises who collaborate on formulating decisions. We will show how in repeated interactions, medical teams cultivate a collection of stable, agreed upon orientations towards evidence and knowledge that establishes an inter-subjective framework within which claims and interpretations can be justified and decisions can be arrived at and shared by others. Medical images, such as X-rays and magnetic resonance imaging (MRI) scans, play an important role in these assemblages of distributed knowing. 


\subsection{Methods}

We have analysed clinical decision-making within a medical expert team involved in diagnosis and treatment of patients with a complex disease called pulmonary hypertension, over the course of nine weeks. Pulmonary Hypertension $(\mathrm{PH})$ is a rare and life-shortening disease characterised by an elevation of blood pressure in the pulmonary artery and an increased resistance in the pulmonary vasculature (Barst 2008). This results in an enlargement and decreased function of the right heart ventricle that causes breathlessness and limitation of exercise capacity that may be very severe (Kiely et al. 2013). $\mathrm{PH}$ has a definition given in terms of a measure produced by an invasive test of right heart catheterisation. It is further classified into five different categories (with a number of subdivisions) according to cause, ${ }^{36}$ for which different treatments are required (Galie et al. 2016). Images play a crucial role in establishing the cause of particular cases of $\mathrm{PH}$ and are therefore important diagnostic tools. A careful clinical history and a range of investigations are required to diagnose and categorise $\mathrm{PH}$. The treatment regime for the patient is based upon these tests and classifications and can range from drug treatments to heart and/or lung transplantation.

The medical team in our research worked in one of eight expert centres in the United Kingdom and Ireland that diagnose and treat $\mathrm{PH}$ and consists principally of pulmonary clinicians, a cardiologist, a nurse consultant, specialist pharmacists, radiologists, junior doctors, specialist nurses and a ward nursing team. Although a study of clinical decision-making is not complete without patients, patients were not included in this particular study for pragmatic reasons alone, as our ethical clearance did not extend to them. For this reason, the study focuses on how images are used for diagnosis, which is an aspect of the decision-making process where the division of epistemic labour falls more on the clinical team; a fuller study will also consider patients (Whitney 2003). Participants in the study were all members of the clinical team and invited to contribute to the study and/or collaborate on it. The team confers weekly in a ward MDT meeting discussing the management of the current ward patients and a radiology MDT meeting where current inpatients, patients in short-stay admissions for diagnostic testing, and outpatients may be discussed. Data were collected through observing weekly MDT meetings; performing eleven qualitative semi-structured interviews with members of the clinical team, and

36 1) Pulmonary arterial hypertension ( $\mathrm{PAH})$ either idiopathic or associated with other conditions, 2) $\mathrm{PH}$ due to left heart disease, 3) $\mathrm{PH}$ due to lung diseases and/ or hypoxia, 4) chronic thromboembolic $\mathrm{PH}$ and

5) $\mathrm{PH}$ with unclear and/or multifactorial mechanisms 
conducting a group discussion on emerging imaging technologies. MDT meetings were not video or audio recorded as we did not have ethical clearance for this; we recorded our observations in notes. In addition, we video-recorded a session of two radiologists collaboratively reporting an X-ray CT scan, and an interdisciplinary meeting to determine the usefulness of an emerging imaging technique. All recordings were transcribed and coded using NVIVO (QRS international Pty Ltd. version 10, 2012). The date used for this particular study are the semi-structured interviews, which had framed our data collection in terms of expertise, teamwork and the role of imaging technologies. The interviews were divided into three main sections: the interpretation and use of images, expertise and trust, and the introduction of new imaging modalities. The analysis of the data broadly followed these categorisations, but also looked for connections between them, using a grounded approach, that is, using the main topics and subtopics of the interviews as a first iteration, and an open coding approach, looking for relationships and groupings within and among these topics and subtopics, thereby establishing recurring and contrasting motifs and themes. In particular, we looked for connections between the MDT observations and the interviews with individual research participants, as is evidenced in the discussion below.

\subsection{Results}

\subsubsection{Social knowing in clinical decision-making}

Decision-making in the $\mathrm{PH}$ clinic involves combining evidence from heterogeneous sources, such as the patient's history, clinical examination, lab tests, images and measurements, awareness of personal and social circumstances, observations of the patient by clinicians on ward rounds and by ward staff or interactions with family. One of the key epistemological challenges of clinicians is to develop an account of every individual patient based on the available evidence, a process which involves the interpretation and adjustment of pieces of evidence so that they form a cohesive and consistent 'picture ${ }^{37}$ of that patient (van Baalen and Boon 2015). These pieces of evidence are generated and interpreted by different people; for example, radiographers generate images by operating the imaging apparatus when the patient is scanned, and by doing initial data processing - which is in a sense, already a first form of interpretation; radiologists interpret the images, but so too do clinicians, with different levels of expertise. Nurses and clinicians generate evidence through the patient history and clinical relationship, and their interpretation provides

37 We use the term 'picture' as it is used colloquially in the domain of our fieldwork, without any commitment to representationalism. A non-representational account of pictures is not at all unusual; see footnote 7 . 
the clinical questions that radiologists use to direct their interpretation of the images. In these respects, the process is distributed over people, working in different and overlapping contexts, at different points of the patient's encounter with the clinic. Part of the adjustment and interpretation of evidence occurs within interactions with different experts, who from their different expertises provide a specific outlook on evidence while fitting this in with other evidence requires the interpretations of other experts. MDT meetings play an important role in socially distributed knowing, for instance, by providing a space where information and interpretations can converge into a shared team decision, as also described by Maseide (2006) and Cicourel (1990).

In our epistemological analysis of team decision-making in the $\mathrm{PH}$ service, we will demonstrate how MDTs need to combine the individual expertises of team members to be able to fit together all relevant information that leads to a team decision and that therefore knowing in distributed.

\subsubsection{Multidisciplinary team (MDT) meetings}

We observed two types of MDT meetings: on the ward and in the radiology section. Here we focus on the second of these. In weekly radiology meetings, the $\mathrm{PH}$ team of our field study reviews the imaging of all patients in the last week, admitted for diagnostic testing, or admitted for acute management of a deteriorating clinical condition, and patients who are being (re)evaluated as outpatients. See Figure 5.1 for an overview of the seating in radiologist MDTs. Radiologists have prepared the meeting by reporting the available images and take a place behind the workstation to navigate the different images using a patient archiving and communication system (PACS). Images are shown at a large screen in front of the room, while clinicians (consultant respiratory physicians and a consultant cardiologist) take place at a table at the front of the room, consulting the medical records of the patients. Other attendees at MDTs are the radiographers who have scanned last week's patients, junior doctors, registrars and visitors. At most meetings at least two radiologists and two consultant clinicians are present. A usual interaction concerning imaging of one patient is structured as follows: the clinician opens with an introduction of the patient, a summary of the previous course of the disease, clinical signs and symptoms, other test results and sometimes a specific question. The radiologist then draws up the images, compares results from different imaging modalities, and compares current images with earlier images if available. They show specific findings in the images and sometimes ask for clarification about the patient's clinical history from the clinician to refine their evaluation. After a series of interactions, the radiologist summarises his or her view, with a response to the initial question, after which the clinician concludes the interaction by 
making a note of the shared conclusion and the follow-up plan for that patient, which they say out loud while writing it down in the patient's clinical record.

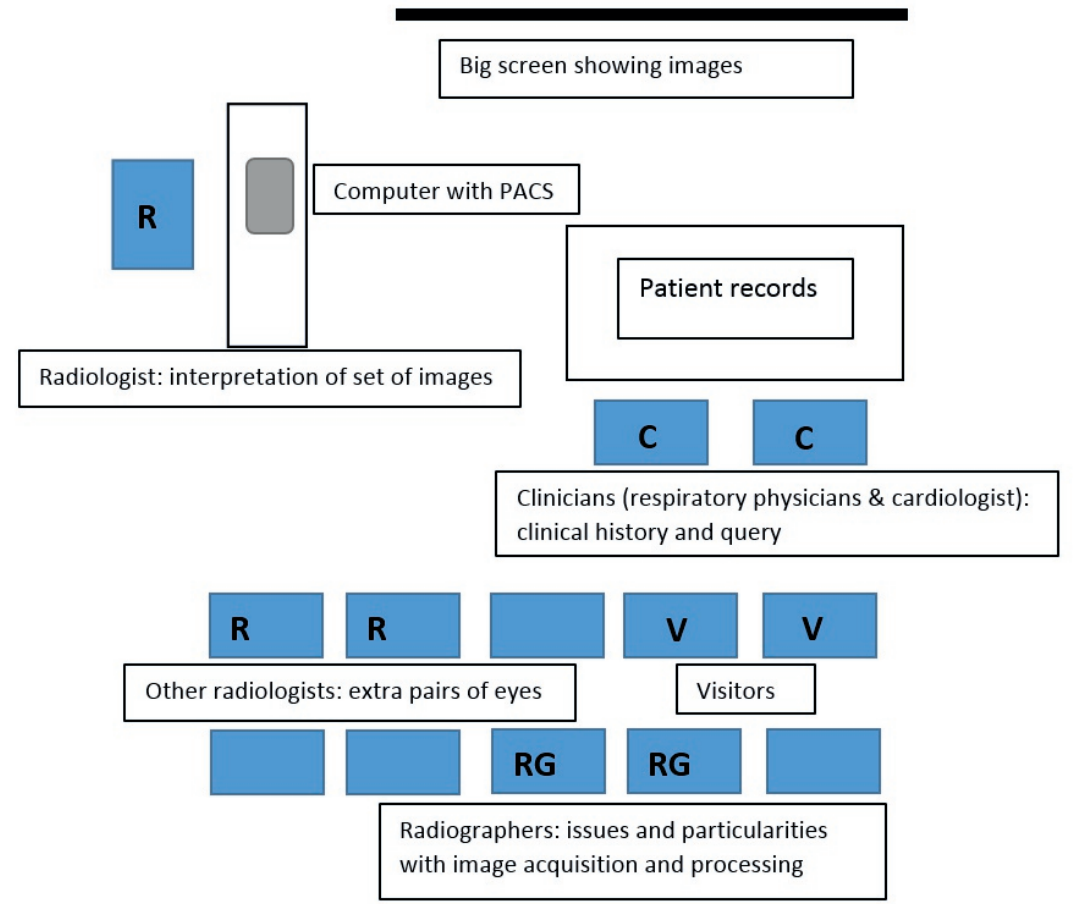

Figure 5.1: Room layout and contributions at weekly radiology multidisciplinary team meeting. PACS, patient archiving and communication system.

In these interactions, heterogeneous sources of knowledge and information are fitted into a group decision by hearing voices that represent a range of expertises. Team members have different knowledge and skills that stem from different backgrounds in training, but different experts also interact differently with individual patients, their bodies and medical instrumentation such as imaging. For example, the clinician has met the patient, has taken his or her medical history, performed a medical exam (e.g., listened to their heartbeat using a stethoscope) and has studied lab results. Radiologists receive only a short summary of the patient's history and the clinician's query, but spent much time studying and interpreting the imaging results. Clinicians decide whether a patient will go for imaging or not, which images modalities are used and which questions are asked, based on their knowledge of the patient and in the context of a diagnostic and management process aimed at understanding and treating an individual to improve symptoms and prolong life. Radiologists are guided by these questions, without having full access to how the cli- 
nician came to that question or what were their reasons for requesting a certain imaging exam for this patient. Conversely, physicians are guided by radiologists' interpretation of their patient's images to make clinical decisions, without having full access to their interpretation, or the expertise that leads them to make the interpretation (see Quote 1).

\section{Quote 1}

"Obviously, you can't read the entire patient notes. So you want a clear summary of what the patient has. Brief. And the best thing is if you've got a clear question of what you want to ask from the imaging. Sometimes the imaging... you could come to multiple conclusions, but if you've got a clear question that makes it a lot easier because you can answer that question and then everything else can be kind of incidental finding, if you find other things." (P3, radiologist)

In short, within these groups, the epistemic labour is distributed over different specialisms and experts, each with different roles and epistemic contributions. For example, radiologists are experts in the way anatomical or pathological structures appear on different imaging modalities, whereas clinicians have a complex understanding of pathological processes and how these present as signs and symptoms in patients. In exchanges in MDT meetings, these different expert approaches help interpret the separate pieces of evidence in relation to other available evidence, providing a refinement and enrichment of these interpretations that a single expert would not be able reach on their own (e.g., see Quote 2). Even though there are different roles and expertises, they must overlap sufficiently for the interactions among members to be meaningful; for example, clinicians in this group described themselves as having more expertise on the images relating to $\mathrm{PH}$ than other radiologists who do not specialise in this area. What is generated is a palimpsest of overlapping and superimposed knowledge rather than a jigsaw made up of discrete pieces that fit together.

\section{Quote 2}

"And that's why an MDT environment is so important... because... you need to have those cautionary people who understand the limitations, so usually the radiologists, to be able to advice the clinical team, about your level of confidence." (P8, radiologist)

These refined and enriched interpretations help to bring heterogeneous information together, into a shared framing of the patient, a collective understanding of the patient's 
illness that is built up from all pieces of evidence, where exchange between different expertises is necessary in order to adjust and reinterpret the available evidence in order to make them fit. Building this shared 'picture' of the patient allows them to come to a shared conclusion concerning the diagnosis and treatment for him or her, which is usually written down in the patient's clinical record while being voiced out loud by the consultant clinician. This voicing out loud underscores the shared ownership of the team conclusion and decision (see Quote 3).

\section{Quote 3}

"Actually within our services it's rather more about you take the opinions of your colleagues and the knowledge that you have as a team, and you work out how to apply that with yourself as an instrument of the team. So you're not placing yourself above anything, but it's more that you're filtering, taking in all things that you're told and trying to work out the best fit." (P1, consultant respiratory physician)

MDT meetings are geared toward consensus within the full team. In most meetings, more than one radiologist and more than one clinician are present, to have an extra pair of eyes and make sure that things are not missed, and also because a conclusion or interpretation that is shared by others is considered more reliable than when it is reached by a single person. Hence, one of the purposes of MDT meetings is to deal with uncertainty and incomplete information, which is a challenges of clinical decision-making (Montgomery 2006). The consensual nature of this process is one way of managing responsibility for the patient, and we would expect that distributed knowledge also leads to distributed responsibility. Members of the unit frequently mentioned that working in a team gave them more confidence in their decisions, see for example Quotes 4 and 5. ${ }^{38}$

\section{Quote 4}

"That's why it's really good we have group of at least two or three radiologists in the team. So we take consensus from the others as well, from the imaging. And if they all agree, than it's more reassuring and then we can say on our report or in the discussion, so for imaging this is what it is" (P4, radiologist) of the hospital; however there are tensions between this ultimate legal and organisational responsibility and the consensual nature of the decision-making process. These are interesting and important issues, but space does not allow them to be explored here. 


\section{Quote 5}

"Usually people come to a consensus. Ehm... and.... it's not just the two radiologists either, because there's a lot of expertise in the imaging from the clinicians as well. So we have like a collective... opinion." (P3, radiologist)

Hence, the process of fitting together heterogeneous sources of information into a coherent and consistent framing of a patient is a collaborative effort, and MDT meetings are an important place where information can be shared, discussed, interpreted and adjusted, allowing the development a collective way of seeing and a shared language. For example, after voicing the team's decision, the consultant clinician also mentions the right heart catheter measurements, which allows for a final check and last integration of all evidence, and which helps radiologists to get a feel for the correlation between the imaging and right heart catheter findings. MDTs continue to play their role when clinicians or radiologists are not in a meeting, first by knowing that they are held accountable for the quality, relevance and comprehensiveness of the information they provide at MDT meetings and second by shaping the information that is gathered in such a way that it fits the structure of the shared framework of the MDT.

In terms of epistemological responsibilities, it is interesting that these include responsibilities towards the sociability of the team. Team members have an epistemological responsibility to weigh up evidence according to their knowledge and also to open up their deliberation to others, justifying to others how they come to a certain interpretation while being sensitive to deliberations and interpretations from others. If we take an overarching epistemological responsibility of each person to be towards a sound shared decision regarding the care of patients, an aspect of that epistemological responsibility is inherently social in character. This means that it is not an epistemological plus a social responsibility, side by side, but both at the same time. This is seen in the more detailed example of image interpretation in the next section.

\subsubsection{Distributed knowing in image interpretation}

The structure of distributed knowing is especially clear when dealing with images. Kelly Joyce (2005) demonstrated how the use of MRI is local, embodied and contingent, for three reasons (Joyce 2005). Firstly because in the production of the image parameter choices by radiographer while scanning a patient influence the resulting image, secondly because the interpretation and translation of the image by trained radiologists produces a report that remains open to divergent interpretations and lastly because imaging can conflict with other available information and can be taken up by the clinician in various 
ways. No one in the PH team in our field work has complete knowledge of the images. Although radiologists are considered the experts when it comes to medical images, they perform their role within an assemblage of other medical professionals and instruments. As argued above, to fully evaluate imaging, it is necessary to fit with other evidence and interpretations of this evidence by other experts, such as the clinical story as provided by the clinician. In addition, important components in this knowledge-generating assemblage are the physical MRI, the work of the radiographers who operate the scanner and instruct the patient and subsequently process the data to produce high-quality images and metrics of the right kind, and software systems such as PACS to share, view and analyse imaging and to add reports. None of these components, clinicians, radiologists, radiographers, scanners and software, can be omitted from an account of knowledge generation in the context of PH diagnosis.

For example, the $\mathrm{PH}$ team make use of an MRI scan called cardiac magnetic resonance (CMRI) to assess the anatomy and function of the cardiac chambers. A typical CMRI sequence requires synchronisation of MRI information with a person's heart rhythm as measured via electrocardiogram (ECG) for as long as 40 minutes, which enables reconstructing a moving image of the heart during the cardiac cycle (this process is called 'cardiac gating'). This image sequence resembles a beating heart, and this is used to assess the function of the heart by visual assessment of chamber anatomy, contraction and potential leaking of heart valves. The right heart function is relevant for prognosis and disease severity, whilst the left heart is assessed to exclude left heart disease. In addition, images are processed to quantify predetermined parameters relevant to cardiac function, such as ejection fraction (the amount of blood pumped by the heart with each heartbeat) and calculated cardiac output (the amount of blood pumped per amount of time). For example, to measure the right ventricular ejection fraction, a measure held to be clinically important by correlating with disease severity and prognosis, the volume of the right ventricle is measured at two moments in the cardiac cycle: immediately before contraction (the end-diastolic volume) and immediately after contraction (the end-systolic volume). This is done by drawing the contour of the right ventricle in all slices covering the right ventricle volume for the two points in the cardiac cycle (Swift et al. 2012a). A radiographer draws the right ventricle contours after which a software program calculates the ejection fraction and other metrics characterizing the right ventricle function that are summarized in a report containing numbers and diagrams that the radiologists receive in PACS.

The above description of the production of one MR metric employed to make clinical decisions demonstrates how knowing in clinical practice is socially distributed. Radiographers make a knowledge claim by drawing the contour of the right ventricle, defining 
which part of the image refers to the ventricle wall and which to the inside of the ventricle. They are able to make such a knowledge claim because they have developed a way of looking at these images, as part of their education and experience, but more importantly in interaction with other experts, such as radiologists and researchers, who establish the relevance of this knowledge claim - demarcating the border of ventricle wall - by relating it to a clinically relevant metric - the right heart ejection fraction. For radiologists to evaluate the right ventricle ejection fraction, and thus make a knowledge claim about disease severity or prognosis, they require knowledge claims made by radiographers regarding the right ventricle wall in order for the measure to be processed. The two types of knowledge claims develop in tandem with each other, through iterative cycles during which the border of the right heart ventricle is picked out for clinical relevance, and the radiographers draw the border in such a way that the radiologists can use it for their decisions. In short, knowledge in this small instance is distributed over at least two people who both play a crucial role in generating an evaluation of the right heart function, and who both play their roles embedded within a broader team and other interactions, with other radiographers, radiologists and consultant clinicians. No one person has complete knowledge of the images, and the knowledge each does have is in virtue of their interactions with others. Each person is responsible for how he or she contributes to the knowledge of others and not only to their own piece of the puzzle. This contribution includes responsibilities towards openness to others and recognition of oneself as part of a team.

\subsubsection{The mediating role of imaging technologies}

In our field study, the physicists, radiographers and radiologists involved in $\mathrm{PH}$ imaging are highly specialized and have a long history of collaboration, developing methods to analyse and evaluate CMR images and metrics together. The technologies, for example, the scanner, the sequences facilitating the acquisition of CMR images, the image processing algorithms and the software tools that enable drawing the right heart ventricle contours, calculating the ejection fraction and sharing the results, play a crucial and active role in these processes. The technologies, the users, the ways of looking, and the possible knowledge claims coevolve with each other. Kelly Joyce (2006) demonstrates how MRI coevolved with ways of looking by describing its historical development. MRI was originally developed as a tool to measure the composition of materials in physics and chemistry (spectroscopy) and later, driven by the 'war on cancer' in the United States, attempted to be modified into a tool to measure tissue composition and ultimately into an imaging method. The images produced by MRI were initially in full colour and included an array of numbers. After being taken up by radiology, MRI scans were presented in grey scale, fitting the images radiologists were already familiar with and the existing technological constraints (Joyce 2006). In the field of PH, this coevolution is also evident. 
MRI, by producing a specific type of contrast, between different types of soft tissues, drives a specific kind of visualisation of the heart muscle, and the method of electrocardiogram-gating allows visualisation of movements of the heart during a complete heart cycle, enabling CMRI. Clinicians and radiologists involved in diagnosis and treatment of $\mathrm{PH}$, from being familiar with heart anatomy and physiology, recognise the relevant structures (i.e., septum, ventricles and valves), and from being familiar with what type of information is required in clinical practice, they recognize which relevant questions might possibly be answered by these types of imaging. However, they need to learn how to recognize deficiencies and how to evaluate function by relating images to clinical outcomes. Together with an ongoing and rigorous discussion, these interactions among radiographers, radiologists and clinicians, and the imaging technologies push the development and tweaking of acquisition sequences to improve image contrast for those specific practices, and image processing and analysis algorithms to produce relevant metrics such as right heart ventricle ejection fraction.

In repeated interactions, medical teams cultivate a collection of stable, agreed upon orientations towards evidence and knowledge that provides an intersubjective framework within which claims and interpretations can be justified and decisions can be arrived at and shared by others. Medical images play an important role in the building of these intersubjective frameworks in three ways: first as enablers or tools in acquiring information; second as communication facilitators, and third as pervasively framing the epistemic domain (Carusi 2008; Verbeek 2008, 2011). Through these three mediating roles cumulatively and simultaneously, imaging modalities are active shapers of the epistemic domain, for example, by shaping what counts as evidence for specific diagnoses, and by shaping classificatory structures and treatment régimes for diagnoses.

That images are enablers or tools in acquiring information is clearly evident throughout the history of imaging, as from the first X-ray, images have been a powerful means of pushing back the limits of observation. In the $\mathrm{PH}$ field, continued research on imaging such as CT and MRI has allowed a visual detection of several mechanisms causing PH (e.g., chronic blood clots in the lung, lung emphysema, left heart disease, etc.) leading to more reliable clinical categorisation and development of specific diagnosis and treatment approaches according to $\mathrm{PH}$-group. Because images play such a crucial role in the diagnosis and management of the disease, they play a prominent role in interactions and 
communications among the members of the medical team. ${ }^{39}$ This is obvious in the radiology MDTs, where the images are discussed; but also clear in the ward MDT meetings. These are led by different consultant clinicians in different weeks, and there is variation in the display and reference to displayed images depending on which consultant clinician leads; mostly the images relating to the patient discussed are displayed and discussed; at the very least, they are always mentioned and referred to. ${ }^{40}$

The third mediating role of images, as pervasively framing the epistemic domain, is closely related to its other two roles, but relates to the sheer scale of image use and research in the domain. MRI became routinely used in the PH unit that we studied since $2004 .^{41}$ Since its introduction, even artefacts of CMR imaging have been found to be diagnostically useful, as in the case of the so-called black blood artefact (Swift et al. 2012b), and other imaging technologies and other tests become less used. The introduction and development of a new imaging technique or modality usually runs alongside and piggybacks on an existing one, as it needs both to cohere with and go beyond the existing techniques and modalities. As we have discussed, the ability to interpret and use the images co-evolves with the development and implementation of the technology, and the expertise and skill with which images are interpreted are built up through continued use over lengthy periods of time, in interaction with other people. When an imaging modality overcomes a critical point and becomes dominant, it pushes out other pre-existing modalities. For example, in Quote 6 there is a radiologist describing how MRI perfusion in combination with accurate CT pulmonary angiography came to be used in their unit as an accurate, non-invasive test that ultimately replaced the more invasive pulmonary angiogram:

39 Images might also play a role in the interactions between clinicians and patients, however, we did not study these interactions in our field work.

40 An imaging modality that plays in important role in $\mathrm{PH}$ diagnosis is the echocardiogram, but interestingly this modality is hardly ever referred to, let alone displayed at MDT meetings. This probably has to do with the fact that echocardiograms are not shared via the same PACS system, with the user-specificity of US images and that interpretation of these images requires cardiologic expertise. Another modality that is relatively little referred to is electrocardiography (ECG).

41 The development of MRI methods for PH diagnosis and classification and the impact of these is another very interesting topic, but goes beyond the scope of this paper and will be discussed in other papers. 


\section{Quote 6}

P4:"...so looking at perfusion you could see the lungs taking up contrast. So if it's even in both sides of the lungs, then that's fine. But if you see like defects, you know like big chunks out of contrast that's missing. Than that's a feature of chronic thromboembolic disease. So we started noticing those.. changes."

[...]

P4: "before, we used to use, just contrast angiography, so what we used to was, to look at the pulmonary arteries, we used to just inject contrast and then just look at the flow of the contrast in the pulmonary artery. So very... it's an invasive procedure, so you have to have a catheter put in the groin and... but, that's more or less obsolete these days. So we don't, we hardly do one a year."

Interviewer: "do you notice anything... do you miss anything about images that.... modalities that you, you know... is there any time that you would say, well we could have seen that on...[a pre-existing modality]"

P4: "the thing is, because we don't do it that often, we're losing the skill to interpret the..."

[...]

P4: "So, you know, if somebody gives us a pulmonary angiography now, I think we'll all struggle to identify what's happening."

When imaging modalities became so embedded into the epistemic domain, it becomes difficult to get an external vantage point on them, and they, in their turn, become the standards against which continued imaging developments are assessed. The evidence for defects in the lung, for example, comes to be constituted by how this is visualized in perfusion MRI. In this way, images are pervasive mediators that can reshape the epistemic domain.

Thus, the epistemology of clinical decision-making is also ineliminably technological as well as being social; in fact, these two aspects cannot be divorced from each other, as they are one in virtue of being the other. The most powerful technological means for probing a clinical domain cannot be used, cannot even have meaning for that domain, outside of the social relations through which interpretations are engendered and decisions are grounded. However, this very process of using technologies effectively - which, we have seen, requires expertise and skill that are honed through social interactions - also makes it difficult to arrive at purely external assessments of specific technological developments once they become the norm, because the process of producing the expertise to interpret them can also, paradoxically, remove the ground for making a comparison. 


\subsection{Discussion}

The study we conducted is a small qualitative study of clinical decision-making in diagnosis and treatment of $\mathrm{PH}$, involving only one team in a relatively short frame of time. In this study, we focused on images and the clinical team using and developing them, whereas further studies need to broaden this out to consider others in the process, in particular nurses and patients; in addition further comparative studies of other $\mathrm{PH}$ teams would enable us to discover how specific our findings are to this team. Even so, we believe that it points to some important features of the social epistemology of image-mediated clinical decision-making. We have argued that clinical decision-making is highly social and mediated by technologies, in this case imaging technologies. Imaging, the ability to interpret images, social practices and the epistemic domain codevelop into a sociotechnical epistemic framework in which members of the clinical team exchange, discuss and fit together evidence toward a team opinion. These aspects of clinical decision-making mean that an individualist epistemology is inadequate. Instead, the epistemology of clinical decision making is ineliminably social and technologically mediated.

In this article we have emphasised the social nature of knowledge in the process of coming to a shared way of seeing, or what Friedrich (2010) labelled 'sight style'. This differentiates our account of socio-technological epistemology from the tradition of distributed cognition, as we do not invoke internal or external representations. Of course, the word 'representation' is frequently used in the clinic as elsewhere in scientific contexts, but our emphasis has been on how something comes to be agreed upon as a representation, and we do not take for granted in advance that anything actually is a representation since this assumes that it is already or a priori clear how to interpret it as a representation. On our account, it is the process whereby an image's status as being a representation of some aspect of the clinical situation is established, that is at issue: as in our example, images come to represent the size of the right heart ventricle through an interactional interplay 
between radiologists and radiographers that foregrounds the border of this ventricle and establishes a way of drawing it, rather than this being pre-given. ${ }^{42}$

Furthermore, our analysis implies that it is not enough to focus on the epistemological responsibilities of knowers operating as individuals, but that in order to understand the domain better, we also need to understand how epistemological responsibilities includes responsibilities towards sociability and technological mediation. In other words, epistemological responsibilities of physicians not only includes the gathering, interpretation and fitting together of evidence for each patient, but also an openness towards evidence and interpretations, and knowledge claims made on their basis by other team members, and making one's own interpretations accessible to others.

The socio-technological epistemology that we are proposing opens up several questions for further investigation: we have pointed to issues about responsibilities of clinical decision-making that need further analysis, as well as issues in the development and validation of new technologies and imaging tools. We end on a note regarding the potential of the socio-technological epistemology we propose to open up new roles for philosophers

42 There are further deep differences between our view and distributed cognition that cannot be dealt with here. These are briefly differences on the status of internal representations (our account takes a phenomenological approach and bypasses these entirely); the espousal of many cognitivist accounts of a computational theory or metaphoric framework of cognition, which we do not adopt; and the view that (for example in Cunningham 2014: 187) artefacts are a subset of tools that assist people to perform cognitive functions that they could otherwise perform for themselves. In the same tradition, images and visualisations have also been understood as distributed representations that aid visual thinking and communication in distributed cognitive systems (Gooding 2006). On our account, artefacts, images and visualisations are not only aids to thinking and communication, as this would take into account only the first and second mediating roles described above; rather they have a further active mediating role in establishing a shared way of seeing as a first step to shared modes of thought. The view taken in this article is an extension of nonrepresentationalist accounts of images and models that one of the authors has been systematically developing in several publications, for example Carusi (2016), Hoel and Carusi (2015) (see also further references to Carusi and Hoel publications on this topic in those articles), and Carusi (2012). Nonrepresentationalism about perception and knowledge is not a new position, but was most significantly advanced in philosophy by the phenomenology of perception of Maurice Merleau-Ponty (1962; originally published in 1945); since then it has had numerous proponents, including significant elaborations of the position in social sciences by, for example, Lynch (1988), Goodwin (1994, 1997), Sharrock \& Coulter (1998). In the tradition of cognitive sciences, it is espoused most notably by Noë (2004). 
and social scientists in participating in the formation of clinical teams. Rephrasing John Hardwig quoted earlier in the paper, our epistemological analysis of the social structure of clinical decision-making suggests that attending to the sociability of clinical decision-making is an essential aspect of what 'makes the members of some teams knowers while the members of others are not'. This attention is something that philosophers and social scientists could contribute to the understanding of clinical decision-making. 


\section{References}

Andersen, H. (2013), 'The Second Essential Tension: on Tradition and Innovation in Interdisciplinary Research', Topoi, 32 (1), 3-8 DOI: 10.1007/s11245-012-9133-z.

Andersen, H. and Wagenknecht, S. (2013), 'Epistemic dependence in interdisciplinary groups', Synthese, 190 (11), 1881-98 DOI: 10.1007/s11229-012-0172-1.

Barst, R.J. (2008), 'Introduction', in Barst. R.J. (ed.), Pulmonary arterial Hypertension: Diagnosis and EvidenceBased Treatment (John Wiley \& Sons, Ltd), 5 ISBN: 9780470059722 DOI: 10.1002/9780470997390.ch1.

Braude, H. D. (2009), 'Clinical intuition versus statistics: different modes of tacit knowledge in clinical epidemiology and evidence-based medicine’, Theor Med Bioeth, 30 (3), 181-98 DOI: 10.1007/s11017-0099106-4.

Carusi, A. (2008), 'Scientific visualisations and aesthetic grounds for trust', Ethics and Information Technology, 10 (4), 243-54 DOI: 10.1007/s10676-008-9159-5.

Carusi, A. (2012), 'Making the Visual Visible in Philosophy of Science', Spontaneous Generations, 6 (1), 106-14 DOI: 10.4245/sponge.v6i1.16141.

Carusi, A. (2016), 'Modelling Systems Biomedicine: Intertwinement and the 'Real”', in A. Whitehead and A. Woods (eds.), Edinburgh Companion to Critical Medical Humanities (Edinburgh: Edinburgh University Press) ISBN: 9781474400046

Chang, H. (2014), 'Epistemic Activities and Systems of Practice: Units of Analysis in Philosophy of Science After the Practice Turn.', in L. Soler, et al. (eds.), Science After the Practice Turn in the Philosophy, History and Social Studies of Science. (London: Routledge), 67-79 ISBN: 978-0415722957

Cicourel, A.V. (1990), 'The integration of distributed knowledge in collaborative medical diagnosis', in J. Galegher, R.E. Kraut, and C. Egido (eds.), Intellectual teamwork: social and technological foundations (Hillsdale, NJ, USA: L. Erlbaum Associates), 221-42 ISBN: 978-0805805345

Cohen, T. et al. (2006), 'A cognitive blueprint of collaboration in context: distributed cognition in the psychiatric emergency department', Artif Intell Med, 37 (2), 73-83 DOI: 10.1016/j.artmed.2006.03.009.

Cunningham, T. V. (2013), 'Socializing Medical Practice: A Normative Model of Medical Decision-Making', (University of Pittburgh).

Fleck, L. (1979), Genesis and development of a scientific fact (Chicago: University of Chicago Press) ISBN: 9780226253251 .

Fleck, L. (1986e [1947]), 'To look. To see. To know.', in R.S. Cohenand and T. Schnelle (eds.), Cognition and fact. Materials on Ludwik Fleck (Dordrecht: Reidel Publishin) ISBN: 978-94-009-4498-5

Friedrich, K. (2010), “Sehkollektiv': Sight Styles in Diagnostic Computed Tomography', Medicine Studies, 2 (3), 185-95 DOI: 10.1007/s12376-010-0050-4.

Friis, J.K.B.O. (2015), 'Gestalt descriptions embodiments and medical image interpretation', Ai \& Society, DOI: $10.1007 / \mathrm{s} 00146-015-0615-6$.

Galie, N. et al. (2016), '2015 ESC/ERS Guidelines for the diagnosis and treatment of pulmonary hypertension: The Joint Task Force for the Diagnosis and Treatment of Pulmonary Hypertension of the European Society of Cardiology (ESC) and the European Respiratory Society (ERS): Endorsed by: Association for European Paediatric and Congenital Cardiology (AEPC), International Society for Heart and Lung Transplantation (ISHLT)', Eur Heart J, 37 (1), 67-119 DOI: 10.1093/eurheartj/ehv317.

Gooding, D.C. (2006), 'Visual Cognition: Where Cognition and Culture Meet', Philosophy of Science, 73 (5), 688-98 DOI: $10.1086 / 518523$.

Goodwin, C. (1994), 'Professional Vision', American Anthropologist, 96 (3), 606-33 
Goodwin, C. (1997), 'The Blackness of Black: Color Categories as Situated Practice', in Lauren B. Resnick, et al. (eds.), Discourse, Tools and Reasoning: Essays on Situated Cognition (Berlin, Heidelberg: Springer Berlin Heidelberg), 111-40 ISBN: 978-3-662-03362-3 DOI: 10.1007/978-3-662-03362-3_6.

Hardwig, J. (1985), 'Epistemic dependence', The Journal of Philosophy, 82 (7), 335-49 DOI: 10.2307/2026523

Hardwig, J. (1991), 'The role of trust in knowledge', The Journal of Philosophy, 88 (12), 698-708 DOI: $10.2307 / 2027007$

Hindmarsh, J. and Pilnick, A. (2002), 'The tacit order of teamwork', The sociological Quarterly, 43 (2), 139-64 DOI: $10.1111 / j .1533-8525.2002 . t b 00044 . x$.

Hoel, A.S. and Carusi, A. (2015), 'Thinking Technology with Merleau-Ponty', in R. Rosenberger and P.P. Verbeek (eds.), Postphenomenological Investigations: Essays on Human-Technology Relations (Lanham, Boulder, New York, London: Lexington Books), 73 ISBN: 9780739194362

Hutchins, E. (1995), Cognition in the Wild (MIT press) ISBN: 0262082314.

Joyce, K. (2005), 'Appealing Images: Magnetic Resonance Imaging and the Production of Authoritative Knowledge', Social Studies of Science, 35 (3), 437-62 DOI: 10.1177/0306312705050180.

Joyce, K. (2006), 'From numbers to pictures: The development of magnetic resonance imaging and the visual turn in medicine', Science as Culture, 15 (1), 1-22 DOI: 10.1080/09505430600639322.

Kennedy, A. G. (2013), 'Differential diagnosis and the suspension of judgment', J Med Philos, 38 (5), 487-500 DOI: $10.1093 / \mathrm{jmp} / \mathrm{jht} 043$.

Khushf, G. (1999), 'The Aesthetics of Clinical Judgment: Exploring the Link between Diagnostic Elegance and Effective Resource Utilization', Medicine, Health Care and Philosophy, 2 (2), 141-59 DOI: 10.1023/a:1009941101276.

Kiely, D. G. et al. (2013), 'Pulmonary hypertension: diagnosis and management', BMJ, 346, f2028 DOI: 10.1136/ bmj.f2028.

Koschmann, T. et al. (2011), "'Can you see the cystic artery yet?" A simple matter of trust', Journal of Pragmatics, 43 (2), 521-41 DOI: 10.1016/j.pragma.2009.09.009.

Koschmann, T. et al. (2007), 'Formulating the Triangle of doom', 7 (1) DOI: 10.1075/gest.7.1.06kos.

Loughlin, M. (2008), 'Reason, reality and objectivity--shared dogmas and distortions in the way both 'scientistic' and 'postmodern' commentators frame the EBM debate', J Eval Clin Pract, 14 (5), 665-71 DOI: $10.1111 / j .1365-2753.2008 .01075 . x$.

Loughlin, M. (2009), 'The basis of medical knowledge: judgement, objectivity and the history of ideas', J Eval Clin Pract, 15 (6), 935-40 DOI: 10.1111/j.1365-2753.2009.01318.x.

Maseide, P. (2006), 'The deep play of medicine', Communication \& Medicine, 3 (1), 43-54 DOI: 10.1515/ CAM.2006.005.

Merleau-Ponty, Maurice and Smith, Colin (1996), Phenomenology of perception (Motilal Banarsidass Publishe) ISBN: 8120813464.

Montgomery, K (2006), How Doctors Think: Clinical Judgment and the practice of medicine (New York: Oxford University Press) ISBN: 9780195187120.

Noë, A (2004), Action In Perception Bradford Book, eds H. Putnam and N. Block (Representation and Mind; Cambridge, London: The MIT Press) ISBN: 978-0262640633.

Perini, L. (2012), 'Image Interpretation: Bridging the Gap from Mechanically Produced Image to Representation', International Studies in the Philosophy of Science, 26 (2), 153-70 DOI: 10.1080/02698595.2012.703478.

Rosenberger, R. (2009), 'A Case Study in the Applied Philosophy of Imaging: The Synaptic Vesicle Debate', Science, Technology \& Human Values, 36 (1), 6-32 DOI: 10.1177/0162243909337117.

Semczyszyn, N (2010), 'Signal into vision, medical imaging as instrumentally aided perception', (The University of Britisch Columbia). 
Sharrock, W. and Coulter, J. (1998), 'On what we can see', Theory \& Psychology, 8 (2), 147-64 DOI: $10.1177 / 0959354398082001$.

Solomon, M. (2008), 'Epistemological reflections on the art of medicine and narrative medicine', Perspect Biol Med, 51 (3), 406-17 DOI: 10.1353/pbm.0.0038.

Swift, A. J. et al. (2012a), 'Diagnostic accuracy of cardiovascular magnetic resonance imaging of right ventricular morphology and function in the assessment of suspected pulmonary hypertension results from the ASPIRE registry', J Cardiovasc Magn Reson, 14, 40 DOI: 10.1186/1532-429X-14-40.

Swift, A. J. et al. (2012b), 'Black blood MRI has diagnostic and prognostic value in the assessment of patients with pulmonary hypertension', Eur Radiol, 22 (3), 695-702 DOI: 10.1007/s00330-011-2306-0.

Tonelli, M. R. (2006), 'Evidence Based Medicine and Clinical Expertise', Virtual Mentor: Ethics Journal of the American Medical Association, 8 (2), 71-74 DOI: 10.1001/virtualmentor.2006.8.2.ccas1-0602.

Tonelli, M. R. (2010), 'The challenge of evidence in clinical medicine', J Eval Clin Pract, 16 (2), 384-9 DOI: 10.1111/j.1365-2753.2010.01405.x.

van Baalen, S. and Boon, M. (2015), 'An epistemological shift: from evidence-based medicine to epistemological responsibility', J Eval Clin Pract, 21 (3), 433-9 DOI: 10.1111/jep.12282.

Verbeek, P.P. (2008), 'Obstetric Ultrasound and the Technological Mediation of Morality: A Postphenomenological Analysis', Human Studies, 31 (1), 11-26 DOI: 10.1007/s10746-007-9079-0.

Verbeek, P.P. (2011), 'Expanding Mediation Theory', Foundations of Science, 17 (4), 391-95 DOI: 10.1007/s10699011-9253-8.

Wagenknecht, S. (2015), 'Facing the Incompleteness of Epistemic Trust: Managing Dependence in Scientific Practice', Social Epistemology, 29 (2), 160-84 DOI: 10.1080/02691728.2013.794872.

Whitney, Simon N. (2003), 'A New Model of Medical Decisions: Exploring the Limits of Shared Decision Making', Medical Decision Making, 23 (4), 275-80 DOI: 10.1177/0272989x03256006. 


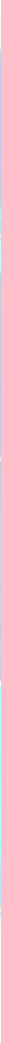




\section{Implicit trust in clinical decision-}

\section{making by multidisciplinary}

teams

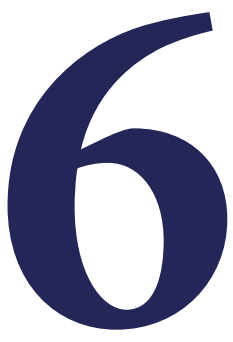

This chapter is published as: S. van Baalen \& A. Carusi (2017) Implicit trust in clinical decision-making by multidisciplinary teams. Synthese. DOI: 10.1007/s11229-017-1475-z

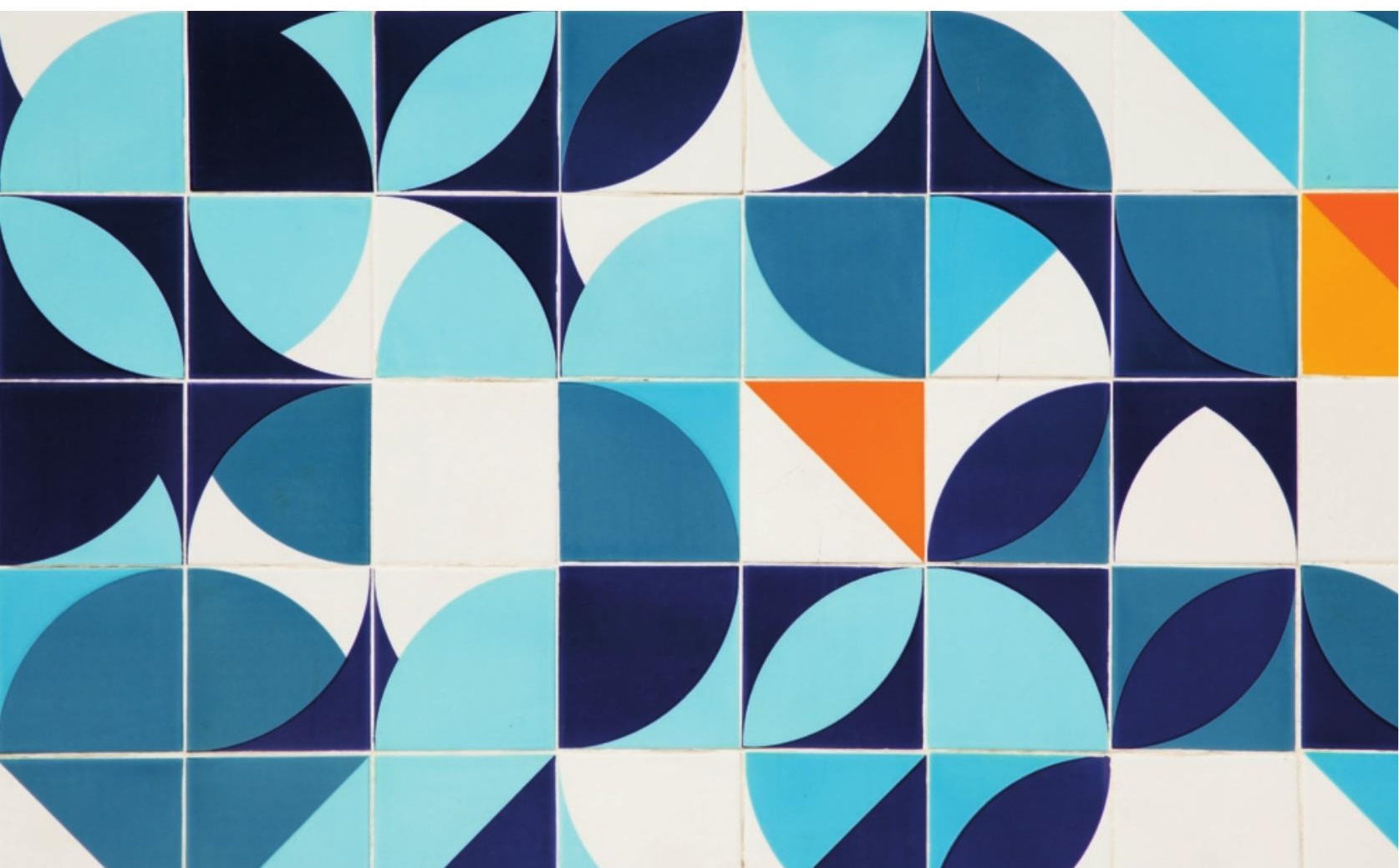




\section{Abstract}

In clinical practice, decision-making is not performed by individual knowers but by an assemblage of people and instruments in which no one member has full access to every piece of evidence. This is due to decision making teams consisting of members with different kinds of expertise, as well as to organisational and time constraints. This raises important questions for the epistemology of medicine, which is inherently social in this kind of setting, and implies epistemic dependence on others. Trust in these contexts is a highly complex social practice, involving different forms of relationships between trust and reasons for trust: based on reasons, and not based on reasons; based on reasons that are easily accessible to reflection and others that are not. In this paper, we focus on what it means to have reasons to trust colleagues in an established clinical team, collectively supporting or carrying out every day clinical decision-making. We show two important points about these reasons, firstly, they are not sought or given in advance of a situation of epistemic dependence, but are established within these situations; secondly they are implicit in the sense of being contained or nested within other actions that are not directly about trusting another person. The processes of establishing these reasons are directly about accomplishing a task, and indirectly about trusting someone else's expertise or competence. These processes establish a space of reasons within which what it means to have reasons for trust, or not, gains a meaning and traction in these team-work settings. Based on a qualitative study of decision-making in image assisted diagnosis and treatment of a complex disease called pulmonary hypertension $(\mathrm{PH})$, we show how an intersubjective framework, or 'space of reasons' is established through team members forging together a common way of identifying and dealing with evidence. In dealing with images as a central diagnostic tool, this also involves a common way of looking at the images, a common mode or style of perception. These frameworks are developed through many iterations of adjusting and calibrating interpretations in relation to those of others, establishing what counts as evidence, and ranking different kinds of evidence. Implicit trust is at work throughout this process. Trusting the expertise of others in clinical decision-making teams occurs while the members of the team are busy on other tasks, most importantly, building up a framework of common modes of seeing, and common ways of identifying and assessing evidence emerge. It is only in this way that trusting or mistrusting becomes meaningful in these contexts, and that a framework for epistemic dependence is established. 


\subsection{Introduction}

In clinical practice, decision-making is not performed by individual knowers but by an assemblage of people and instruments in which no one member has full access to every piece of evidence. This is due to decision-making teams consisting of members with different kinds of expertise, as well as to organisational and time constraints (van Baalen et al. 2016). In this paper we will argue that implicit trust plays a pivotal role in the effective performance of such assemblages and that medical imaging such as X-ray and magnetic resonance imaging (MRI) mediate the cultivation of these trust practices, by enabling the establishment of a shared 'space of reasons'.

In social epistemology, one source of evidence that has been widely discussed is evidence that is provided by testimony from others (e.g., see Lackey 2010). In the debate on the epistemology of testimony the central focus is how testimony can be the basis for justified belief or knowledge, which is traditionally answered in two lines of epistemological theories: reductionist and antireductionist. It is not the primary aim of this article to provide an in-depth discussion of the epistemologies of testimony, but very roughly, these two lines of thought can be characterised as follows: A reductionist account reduces trust to the reasons that support it, and assert that it is reasonable to accept someone's testimony when there is positive evidence of the reliability of testimony, while according to non-reductionist accounts testimony is similar to other sources of knowledge (such as perception or memory) that are basic, and justified unless there is evidence against them (Faulkner 2007; Lackey 2010; Origgi 2004). In addition to reductionist and antireductionist theories of testimony, some epistemologies of testimony claim that features of the interpersonal relationship between speaker and audience provide the epistemic value of the testimonial beliefs acquired (Kappel 2013). For example, several authors argue that speakers can be motivated to act in a trustworthy way when their audience is expecting them to and when betrayal will be associated with negative reactive emotions (Faulkner 2007; Pettit 1995).

The reliance on testimony within teams that collaborate to acquire some shared epistemic goal, such as multidisciplinary clinical teams making diagnosis or treatment decisions or interdisciplinary scientific collaborations, means that individual team members are epistemically dependent on each other (Andersen and Wagenknecht 2013; Hardwig 1985). Epistemic dependence, in turn, brings trust into play, since every member has gaps in their knowledge and abilities that require precisely the leaps of faith that are characteristic of trust (Lagerspetz 2015; Mollering 2001). Philosophers have argued that dealing with epistemic dependence in multidisciplinary teams requires epistemic trust (Hardwig 1991; Wagenknecht 2014). In these understandings of epistemic trust, trust involves dependence 
on another person for some belief or other input in one's reasoning or knowledge production, and an aspect that provides a reason for trusting, e.g., a disposition, an expectation, assumption, or communication in combination with confidence. In this paper we assume epistemic dependence between team members and focus on the reasons for trust in this team.

Trust of all forms fills in a gap between evidence and expectation: it occurs when there is an expectation that one's friend will return money loaned, that the babysitter will conscientiously take care of the children left in his care, that one's doctor has correctly identified which drug to prescribe for a treatment, that one's colleague correctly reports a radiograph, or a myriad other everyday expectations. For example, Guido Mollering claims that trust involves an expectation of a favourable outcome, the interpretation of available evidence and a mental leap enabled by suspension, bracketing the unknowable (Mollering, 2001). This approach accounts for moving from trusting based on a purely rational consideration of 'good reasons' to trust based on fragmentary information as the basis for further action. The gap is not one that can be inductively filled in. Epistemic trust relates to belief and knowledge; whereas moral trust relates to behaviour and actions as falling under the principles of 'right' and 'wrong' (broadly); and even more broadly, social trust is trust that others will comply with social or psychological 'rules', conventions or practices (Lewis and Weigert 1985; Mollering 2001).

Epistemic trust is characterised in many different ways, of which we cannot provide an overview here, but we will give some examples. Klemens Kappel (2011) argues that epistemic trust is a non-inferential disposition of an individual to believe what another individual asserts or transmits. According to Kappel, this can ground knowledge and justification when the disposition is discriminating and defeater-sensitive. Frost-Arnold (2014) argues that "trust involves taking the proposition that the trusted will act as expected as a premise in one's practical reasoning,"(Frost-Arnold 2014, p. 1957). McCraw (2015) argues that for $\mathrm{H}$ to place epistemic trust in $\mathrm{S}$ that $\mathrm{p}$ requires; $\mathrm{H}$ to believe that $\mathrm{p} ; \mathrm{S}$ to communicate that $\mathrm{p} ; \mathrm{H}$ to depend upon $\mathrm{S}$ for (H's belief that) $\mathrm{p}$; and that $\mathrm{H}$ has confidence in $\mathrm{S}$ with respect to $\mathrm{p}$ (McCraw 2015, p. 421).

A question that often arises is whether epistemic and moral trust can be distinguished in practice, or how closely related they might be. For example, in his analysis of epistemic trust, Faulkner (2007) distinguishes between two forms of trust. Predictive trust requires the audience to knowingly depend on the speaker, with an expectation, in the sense of predicting that something will happen (Falkner 2007, p. 464), whereas affective trust also assumes dependence but implies a different type of expectation, namely in the sense of 
expecting something of someone, such as expecting someone to tell the truth. In the latter type of trust, when the trusted person does not show any motivation to do what is expected of them the truster is susceptible to a certain reactive attitude, which is, according to Faulkner, resentment. In response to the objection that affective trust brings in other forms of trust that are not strictly epistemic - such as reasons of friendship or moral reasons (ibid, p. 464). Faulkner argues that affective trust is not moral, strictly speaking, because 'one criminal could affectively trust another to do something immoral' (ibid, p. 464). While it is not clear that a situation that is immoral necessarily contains no morality (such as loyalty), this mistakes the source of the objection, as to describe these as moral reasons does not imply 'moral' as positive evaluation, but rather 'moral' as in the sense of 'falling in the domain of the moral' (just as moral philosophers do not always behave morally). Faulkner argues that when affective trust is epistemic, it is so by virtue of its target: that is, when there is a presumption that another is telling the truth, then affective trust is epistemic. Interestingly, he goes on to write that "In the context of trusting a speaker for the truth, this presumption of trustworthiness provides an epistemic reason because it is the presumption that the speaker is telling the truth. What is right in this objection is that the grounding presumption of affective trust is rarely isolated. Trust is ordinarily motivated by a view of the relationship and values shared with the trusted, or what one desires to share with the trusted," (ibid, p. 464). Faulkner here goes some way to recognising that shared values play an indispensable part in affective trust, but he still wants to distinguish what makes this trust epistemic from these other values in which it is embedded. This is where we part ways. Following philosophers such as Bernard Williams, we would be more inclined to say that affective trust that takes the form of presuming a speaker is telling the truth also involves an expectation of truthfulness on the part of the speaker, and this is a moral quality (Williams 2002).

Another family of theories on epistemic trust characterize it as trust in the competence and goodwill of others, suggesting that epistemic trust also involves moral trust (Hardwig 1991; Origgi 2004). In moral philosophy, trust (and distrust) is often regarded as an emotional rather than cognitive state and includes an expectation about the future: that the vulnerable position you have put yourself in by entrusting someone with something you care about will not be exploited (Baier 1986; Jones 1996; Lahno 2001). There are different views as to how closely moral character and epistemic trust are related. For example, Origgi (2004) argues that moral character is only relevant in the assessment of goodwill and not of competence, and Adler (1994) and McCraw (2015) deny that moral character is involved in epistemic trust at all, claiming that it is possible to trust a person's report without trusting the person (Adler 1994), or that only competence and not goodwill is relevant for epistemic trust (McCraw 2015). Hardwig, instead, stresses that epistemic trust includes 
reliance on the person's moral character, of which an aspect is truthfulness, as well as their epistemic character, which includes competence, conscientiousness and epistemic self-assessment (Hardwig 1991, p.700). ${ }^{43}$ Moral aspects of trust play out in interpersonal relationships. For example, for Steven Shapin, to foreground the social aspects of science is also to foreground its moral aspects (evident throughout (Shapin 1994), but especially in Chapter 1); whereas for others, (such as in the overview by (Lewis and Weigert 1985) social trust is not necessarily moral, though it is normative. In this paper, we will tend to see the interpersonal in the broad moral terms of Shapin's perspective, for expediency in the present context, as we will not explore the kinds of norms social norms are.

A further distinction that is important for our account of knowledge is that between implicit and explicit forms of trust. There are different ways of understanding this distinction, but for our purposes we distinguish between forms of trust that are tacit in a situation of trust, or that trusters are not aware of (and need not be), and forms of trust that are tacit and can be brought to awareness or reflected upon, for example when there has been a disruption in trust (Hertzberg 1988; Lagerspetz 1998). All in all, we are more concerned with this latter form of implicit trust, in order to show how it operates. All forms of trust can be implicit or explicit.

In this article, we focus specifically on expertise, that is, specialised skills and knowledge not shared by all members of a team, and how members of a team who have only partial access to evidence due to differences in expertise face trust issues making everyday decisions. We do not take up the debate between reductionist and anti-reductionist epistemologies of trust, but instead focus on the question what it might mean to have a reason for trust, and aim to show that although team work in medical contexts demands trust based on reasons, in order for there to be reasons that are able to justify trust in the skills and expertise of others, basic forms of trust also need to be in place. The distinctions that are most important for our account are firstly, that between implicit and explicit trust, or as we prefer to put it, between trust with and without awareness; secondly, that between epistemic and moral or interpersonal trust. On this front we argue that while these two forms of trust have different targets, they support each other in actual situations.

'Character' is another word closely associated with ethics, especially in virtue ethics. We will not explore that in this paper, but a next step in the theoretical framework of trust in science would be the moral aspects of professional character. 
The question we have asked of trust in this article cuts across epistemic and moral aspects of trust. Rather than focusing on the rationality of clinical decision-making in team contexts, we focus on having reasons (Toulmin 2001), which holds for both aspects of trust. We accept that trust in contexts of clinical decision-making involves reasons of different kinds, and these may be epistemic (geared towards knowledge) or moral. These epistemic and moral aspects of trust are interdependent and therefore we think that trust in these teams cannot be reduced to either moral or epistemic aspects, but instead that any analysis of trust in team decision-making should not exclude moral aspects of trust. Since the members of clinical teams have a high level of epistemic dependence among one another, trust plays a crucial role in the team being a competent team as well as one made up of competent members, that is a team able to carry out tasks relating to the diagnosis and treatment of patients. Hardwig shifts the normative question about trust from individuals to teams: "Knowing, then, is often not a privileged psychological state. If it is a privileged state at all, it is a privileged social state. So, we need an epistemological analysis of the social structure that makes the members of some teams knowers while the members of others are not." (Hardwig 1991, 697) We agree with Anderson and Wagenknecht (2013) that trust in these contexts is not and ought not to be blind, but is and ought to be reflective, that there ought to be reasons supporting trust. Our question is this: What is it to have reasons to trust someone's expertise, skill or proficiency at a task? And here we argue that there are not clearly reasons in advance of trusting one's colleagues; reasons and trust are instead interwoven.

In this paper, we will elaborate on the trust relations between members of established teams with a history of working together, making collaborative decisions in day-to-day clinical practice, but whose difference in expertise means they are epistemically interdependent. The aim of the paper is to show the crucial role of implicit trust in a complex epistemic team such as a clinical decision-making team, and to shed light on how implicit trust operates: it's 'mechanisms.' The overall framework of our account is that there are two forms of implicit trust at play in teams: moral (that is, trust in the goodwill of others to participate in team work and collaboration, and/or in their professional ethos ${ }^{44}$ ), and trust that is epistemic (that is, trust in the competence, expertise and skills of others, in their epistemic dependability). Within the broad category of implicit trust (either epistemic or moral), we focus on two ways for trust to be implicit: Trust A, implicit in virtue of being without a prior act of reflecting on reasons, or bringing such reasons as there may be into awareness; Trust B, implicit trust by virtue of there being reflection on reasons,

44 See (Oakley and Cocking 2001) for an account of the ethical aspects of professionalism. 
but the reasons are directly about a task at hand, and only indirectly about the epistemic dependability of others. Our paper also focuses on a further aspect of these reasons in Trust B: the lack of reflection is not because they're accepted without question, but rather because the reasons do not precede the team, and therefore are absent until such time as they are established through ongoing interactions and 'doing together' in the team. Our fieldwork shows that the awareness and reflection of team members is directly oriented towards tasks undertaken together; out of this process reasons that can indirectly support (or not) trusting fellow team members emerge and are established.

We stress that our fieldwork is no more than a snapshot of an established team with a long history of collaborative decision-making, and this no doubt plays an role in laying down bases for ongoing trust practices, which obviously extend beyond the bounds of this empirical study. However, it is beyond the scope of any empirical study to establish some form of originary trust, or an $a b$ initio form of trust, on which other trust practices would be founded, since every empirical study would find that trust builds on something else. (The same cannot be said of mistrust consequent to a breach of trust, which can be traced to an event or action, but which also shows what trust was depending upon if this was not explicit.)

We argue that in the process of establishing criteria for jointly carrying out tasks, a shared framework or 'space of reasons' is established (Carusi 2009) within which having reasons for trusting each other's skills, expertise or competence gains traction and meaning. ${ }^{45}$ Crucially, this is a process in time: team members come into already established teams that already have shared practices; they are co-opted or brought into teams by already trusted team members. There is a great deal of implicit dependence on what is already in place, as teams collaborate on every day decision-making, and on developing and embedding new technologies in their practice. These frameworks are developed through many iterations of adjusting and calibrating interpretations in relation to those of others, establishing what

45 The idea of the 'space of reasons' draws upon several philosophical threads: firstly Wittgenstein's On Certainty (1969), where the question of what can count as a test or grounds for knowledge or doubt is raised (for example, Para 109-110), and the suggestion is made that all testing takes place within a system, 'And this system is not so much a point of departure as the element in which arguments have their life' (para 105). The term 'space of reasons' is borrowed from the discussion between Wilfrid Sellars, John McDowell and Robert Brandom (See for example Brandom 1995). The broad claim is that not just anything can count as a reason, but needs to be given intelligibility through interconnected norms and criteria in a context or system. 
counts as evidence for or against a claim, and ranking different kinds of evidence: to have a similar orientation to evidence in a clinical decision-making space is to be in a shared 'space of reasons.' Taking the use of imaging technologies for diagnosis and treatment of a complex disease as our central example, we argue that establishing a common way of seeing or mode of perception is essential to establishing what can count as a reason in the intersubjective framework, as argued by (Carusi 2008), developing the notion of the sensus communis from Kantian aesthetics. Carusi's suggestion that the visualisation software plays an important role in forming this common way of seeing is borne out by Kathrin Friedrich's (2010) analysis of computed tomography images. Friedrich shows that software plays an important mediating role in setting up shared 'sight styles' across a team (Friedrich 2010), drawing upon Ludwig Flecks' notion of 'thought collectives' and 'thought styles' (Fleck 1979, 1986e [1947]). While Friedrich focuses on the software interface of images (the Graphical User Interface or GUI), we focus on the interactions around image processing that lead to images that have the features required for clinicians.

In Paragraph 6.2. we will introduce the domain of our fieldwork, a clinical team working on diagnosis and treatment of a complex disease called pulmonary hypertension (PH). In this domain, the use of imaging is pervasive and therefore it plays an important role in our analysis. In Paragraph 6.3. we introduce our framework of implicit trust through our analysis of interdependence between team members with different expertises. Through an iterative process in repeated interactions team members converge on orientations towards evidence, establishing and cultivating a shared space of reason that provides a framework in which interpretations, reasons and justifications can be shared. In Paragraph 6.4. we argue that imaging mediates the establishment of a space of reasons by developing a shared way of looking and an agreement about what is worth looking at and what claims looking can support. We present an example of image processing of a specific MRI modality to illustrate how this works in practice. We conclude with an overview of the 'mechanism' of implicit trust that we derive from our analysis, and a discussion of issues with epistemological responsibility and consensus that arise from our account.

\subsection{Pulmonary hypertension}

Our account is based on a qualitative study of decision-making in image assisted diagnosis and treatment of pulmonary hypertension $(\mathrm{PH})$, within a medical expert team working in one of eight expert centres in the United Kingdom and Ireland that diagnose and treat PH. The team consisted principally of pulmonary clinicians, a cardiologist, a nurse consultant, specialist pharmacists, radiologists, junior doctors, specialist nurses and a ward nursing 
team and conferred weekly in a ward multidisciplinary team (MDT) meeting discussing the management of the current ward patients and a radiology MDT meeting where current inpatients, patients in short stay admissions for diagnostic testing, and outpatients may be discussed ${ }^{46}$. The clinicians that we have observed had been working together (in slowly changing membership compositions) for years. As each new member comes into the team, he or she is embedded into the already existent team with its well-established practices and forms of interactions. Junior members are trained by senior members; new senior colleagues are recruited by the existing team members; usually wider networks play a role here, such as acquaintance with supervisors or other colleagues of new recruits.

$\mathrm{PH}$ is defined by elevation of blood pressure in the pulmonary artery, the artery through which blood is pumped from the heart to the lungs, as measured by an invasive test called right heart catheterisation, and increased resistance in the pulmonary vasculature. This leads to enlargement and decreased function of the right heart ventricle that causes breathlessness and limitation of exercise capacity and is ultimately life-threatening. It is further classified into five different categories (with a number of subdivisions), ${ }^{47}$ for which different treatments are required. A careful clinical history and a range of investigations are required to diagnose and categorise $\mathrm{PH}$. The treatment regime for the patient is based

46 Data were collected through observing weekly MDT meetings; performing eleven qualitative semi-structured interviews with members of the clinical team and conducting a group discussion on emerging imaging technologies. In addition, we video-recorded a session of two radiologists collaboratively reporting an X-ray computed tomography (CT) scan, and an interdisciplinary meeting to determine the usefulness of an emerging imaging technique. MDT meetings were not video or audio recorded as we did not have ethical clearance for this; we recorded our observations in notes. All interviews were audio recorded, and recordings were transcribed and coded using NVIVO (QRS international Pty Ltd. version 10, 2012). Data for this particular study are the data from MDT observations and semi-structured interviews. The interviews were divided into three main sections: the interpretation and use of images; expertise and trust; the introduction of new imaging modalities. We used a grounded approach for the analysis of the data, broadly following these categorisations, but also looking for connections between them. In other words, we used the main topics and sub-topics of the interviews as a first iteration for analysis of transcripts and notes, and an open coding approach, looking for relationships and groupings within and among these topics and sub-topics, thereby establishing recurring and contrasting motifs and themes, particularly connections between the MDT observations and the interviews with individual research participants.

47 1) Pulmonary arterial hypertension (PAH) either idiopathic or associated with other conditions, 2) $\mathrm{PH}$ due to left heart disease, 3) $\mathrm{PH}$ due to lung diseases and/or hypoxia, 4) chronic thromboembolic $\mathrm{PH}$ and 5) $\mathrm{PH}$ with unclear and/or multifactorial mechanisms 
upon these tests and classifications, and can range from drug treatments to heart and/or lung transplantation.

In the $\mathrm{PH}$ clinic, team members have to combine evidence from heterogeneous sources, such as the patient's history, clinical examination, lab tests, images and measurements, basic textbook knowledge and results from clinical research to form a cohesive and consistent 'picture' of each individual patient based on the available evidence. Part of this process occurs within interactions between different experts, who from their different expertises provide a specific outlook on evidence, for example radiologists interpret images whereas clinicians provide clinical information from the patient's history and physical examination, and nurses from clinical interactions with patients and their family on the ward. Fitting the different interpretations together with other evidence requires the interpretations of other experts. In these respects, the process is distributed over different instruments and people, working in different and overlapping contexts, at different points of the patient's encounter with the clinic generating and interpreting evidence (van Baalen et al. 2016).

Even though there are many different sources of information relating to any particular patient, images play a dominant role in diagnosis and treatment in the team that we studied. A feature of this team is the close inter-relationship between image engineers, radiographers, radiologists and consultant clinicians. ${ }^{48}$ This means that expertises around imaging (producing, interpreting and using them in clinical contexts) are particularly pronounced, giving the opportunity to see how they operate, and how they interplay between expertise and technologies.

48 This team may not be representative of all clinical teams specialized in diagnosing and treating $\mathrm{PH}$ or of any other specialty. For instance, not all clinical team have such intensive collaborations with imaging engineers, and in this set up radiographers usually attended radiology MDT meetings, which is usually not the case for other teams. We did not assess the extent to which this team differs from others and how that impacts the validity of our analysis to other teams. 


\subsection{Implicit trust in a multidisciplinary team}

Clinical decision-making is distributed, combining expertises from a range of professionals contributing specific aspects to the framing of a patient, stemming from different knowledge, experience and skills as well as from different roles in practice. No individual member of the team has full access to every piece of evidence for two reasons: firstly, the evidence is gathered by different people (clinical history and examination by the consultant clinician(s); the echocardiograms and ECGs by the cardiologist(s); the images by the radiographer(s); the reports on the images by the radiologist(s), and so on). This division of labour regarding the gathering of evidence is due partially to pragmatic and organisational constraints. Secondly, the interpretation of evidence, such as ECG measurements or imaging, and the action to be taken due on its basis, such as prescribing a specific drug or recommending an organ transplant, requires expertise that is not available to all team members. As a consequence, every individual team member only has direct access to a fraction of the evidence, and team members are epistemically dependent on each other for the gathering and interpretation of evidence necessary to make clinical decisions (Andersen and Wagenknecht 2013; Hardwig 1985).

This relationship of epistemic dependence was evident throughout our fieldwork. It structurally organises the working week of the PH team, with weekly MDT meetings of two types: the ward MDT meeting and the radiology MDT meeting held on one morning each week, with the second immediately following the first. The ward MDT meeting is led by one of the consultant clinicians (on a rotating basis), with a mix of other members of the team caring for patients in the ward, such as junior doctors, pharmacists, nurses and others. The cases of the week are discussed, and immediately following the ward MDT meeting, one consultant clinician does a ward round, and at least one other consultant clinician (but usually more), attends the radiology MDT meeting where selected cases from the ward MDT meeting are discussed along with others that are already on file for the radiology MDT meeting. The relationship between radiologists and consultant clinicians is a good example of a division of expertise that brings trust into play. There is general acknowledgement that the radiologists are the experts on giving interpretations of the images, over the consultant clinicians, so much so that the consultants do not give a final judgement on the images to patients until such time as they have had a report from the radiologists, as is seen in Quote 1 from a consultant pulmonary clinician. However, this higher level of expertise is accorded to the cardiothoracic radiologists who specialise in pulmonary hypertension and who have been working with the team over many years, and not general radiologists or those not in the team (for example, see Quote 2 from a consultant pulmonary clinician). The consultant clinicians describe their own expertise 
as having been honed by working with the radiologists (for example, see Quote 3 from a consultant pulmonary clinician). However, the interactions among these different expertise groups, which have been developing in some cases over several years, have also created a situation where the expertise of each is developed in dialogue with the other: Quote 4 from a cardiothoracic radiologist and Quote 5 from a consultant pulmonary clinician illustrate this.

\section{Quote 1}

"I think I would always trust the radiology interpretation above and beyond my own. Because... I guess a lot of that comes down to your confidence. Ehm... and your certainty. As I look at more scans, and this is going to be an ongoing thing throughout my working career I guess, I will probably get more experienced and more confident in my own interpretation of scans. And I'm happy with certain degrees of that. And interpretation. But I still... But I still very much value and need, if you like, that to be... double checked by the radiology staff. So I would be very unlikely to formulate a final opinion on a patient without having had the scans reviewed with radiology. I would, I would come to my own conclusion, but I wouldn't finalise that until I'd had that double checked. Which is probably a bit different to certain people, I think it places different strengths and, different people have different degrees of confidence in their interpretation of things. Ehm.. so that's, but that's the way that I think about it." (P10, consultant pulmonary physician)

\section{Quote 2}

"Yea, you'd look at it [a report from a general radiologist] and them sometimes we'd take it back to ours [radiologist from the PH team] and they'd say "that's rubbish". So, I think, you know, we'd... yea, I would look at it. If I get a report, I always look at the scans and make sure that fits in with what... what I'm seeing." (P11, consultant pulmonary physician) 


\section{Quote 3}

P: "So I'm not bad in [interpretation of images regarding] lungs, lung parenchyma I think I'm actually quite good... Ehm... and... the different patterns in gas trapping and clearly we're good at the heart and the pulminovasculature, although we still miss clots occasionally, and stuff like that, so, some expertise. And in some selected areas more expertise than some radiologists, probably. Just in very selected areas. Intereviewer: "So how would you say you obtained that expertise?"

P: "Ehm... just sort of.... experience, reading, ehm... yea... experience and reading your bits and pieces and picking up instruments, pick up tips from the radiologists in the MDT over the years."

Interviewer: "So it's also the interaction with the..."

P: "yea, you also know what they're going to... you know what they... You see an image and you go "Oh, I know they're going to report that." Or what the differential diagnosis is going to be. Because you've seen it many times before." (P11, consultant pulmonary physician)

\section{Quote 4}

Interviewer: "Who do you think are the experts on the images?"

P: "Ehm... I think us radiologists, because we've got the background. And because we're dealing with images on a daily basis. We do... you know we do, we are familiar with what we're doing. But at the same time, I think what we say, or the decision that what we say on the images, does base heavily on what the clinicians come up with as well. So depending on the history, what they think the patient's background is. Then we can narrow our, our findings." (P4, consultant cardiothoracic radiologist)

\section{Quote 5}

Interviewer: "And do you influence each other in that process [...], I don't mean specifically about diagnosis, but just in what you're looking for?"

P: “yes, I think that's fair isn't it. I mean you point people in the... if you sit and work with people for several years, you're educating each other. So radiologists are educating us and we're educating the radiologists." (P7, consultant pulmonary physician) 
In these examples, we have focused on the interactions between radiologists and consultants, both groupings being clinicians but with different expertises. These are not the only forms of expertise in the team that will contribute to the diagnosis, treatment and care of patients, but we focus on them for the moment, firstly because radiologist-consultant interactions are extremely important in a domain where imaging plays a pivotal role, but secondly, they are fairly typical of what we found among other expertises in the group. It is important to note that while all of the examples we have discussed explicitly talk about trust, this is most likely because our questioning purposefully framed the issues in terms of trust. It is notoriously difficult to empirically grasp when people take themselves to be trusting within actual situations, but we wanted to know how our research participants would respond once trust was 'on the table' so to speak. That is, we wanted to know whether they would recognise themselves as trusting, and how they would discuss trust, what kinds of things came up around trust. In fact, all the research participants easily recognised the situations they worked in as situations in which they trusted, and none of the participants repudiated the very category. More interesting were the kinds of reasons that they gave: for example, they readily appealed to expertise (for example Quote 1), but they also pointed to aspects of the tasks they're carrying out: discrepancies between the radiology report and 'what I'm seeing' (Quote 2) (while what they're seeing is very much established in their constant interactions with each other). Quote 6 shows how quickly talk of trust becomes focused on the fine grain of evidence gathering: the discrepancy between the way an MRI 'looks' to the numbers ("the ejection fraction [given quantitatively] is very low ...”.). Quote 6 goes on to show that even when there is reason to be sceptical about something [the numbers], it is not directly about others' skills or competence but because of technical challenges and difficulties. (More about this in the next section). This was typical of our conversations with our research participants, where even in the abstract without naming any particular person, questions about trust were answered very quickly in terms of forms of evidence and how one is weighed up against another. As we shall argue below, this suggests that team members focus directly on the task at hand and only indirectly on reasons for trusting the expertise of others. 


\section{Quote 6}

Interviewer: "What makes you trust people more or less?"

P: "I think your experience, so if you have seen for a long period of time that they make the correct diagnosis, they can probably see things other people can't see. They [the other people] miss. And you trust them [the experience people] more." Interviewer: "It's your personal experience?"

P: "It's a personal experience. For example if you have the right ventricle, now talking about his ejection fraction, what looks to be absolutely normal [on the MRI scan] and the ejection fraction is very low... It's common sense. That's the other thing. It can't be true."

Interviewer: "So it's your own..."

P: "So you have to filter all the numbers, because the numbers... because of the technical challenges and technical difficulties, may be wrong." (P5, consultant cardiologist)

What we draw from these examples is evidence of the extent of interdependence between forms of expertise, and the extent to which trust is based upon familiarity and experience over long stretches of time (see also Jirotka et al. 2005). However, it is also striking that this familiarity and experience with each other goes further than providing a cumulative stock of evidence for trusting the expertise of the other. Rather, the consultant clinicians become better at reading the images as they learn to see how the radiologists would report them, and the radiologists refine their reports on the basis of the queries that the consultant clinicians direct to them. Thus, the consultant clinicians' confidence in the radiologists is not based just on more evidence of their trustworthiness. Rather, it is based on a mutual adaptation, one to the other, whereby the consultant clinicians come to see as the radiologists do (that is, to pick out the same features as they would for a report), and the radiologists bring to the foreground among the indefinitely many features of an image, those features that are important in the consultant clinicians' world. In the next Paragraph, we will give another example of this mutual adaptation, but here we pause in order to consider initial implications for trust.

Among the different members of the team more or less directly involved in diagnosis and treatment decisions, none explicitly mentioned moral trust; one participant among the image engineers mentioned this directly and without our asking or mentioning trust ourselves. See Quotes 7 and 8. This participant points to moral and interpersonal aspects of trust, and also gives reasons. The team members referred to in these quotes overlap with 
those we observed in the clinical teams, and our observations bear out a strong norm of conscientiousness and a shared norm of putting patients first.

\section{Quote 7}

"I think he's pragmatist, he's people person, and I respond to trust. For me it was trust. I like the guy, I'd do anything to help him out. Within reason that I can. And, as clinicians go, he's a very very open, friendly and non-political person, and I kind of warmed to that kind of personality. You can tell with him that he's a... his main priority is patient care." (P9, MRI physicist)

\section{Quote 8}

"We founded our collaboration on a need to get something going, and that's always a good starting point. You work on something and you get it working and you see that it's actually been useful.” (P9, MRI physicist)

The first implication is that the forms of trust described in the interviews and observed in our fieldwork is implicit, in these senses: trust in the skills and expertise of others (for example, as in Quotes 1 and 4) implicitly assumes that those others are not withholding their competence, that they do their work conscientiously and with goodwill and according to shared norms. This is moral/interpersonal trust, generally of the kind we have labelled Trust A (although as we saw in Quote 7, it can also be brought into awareness). There is also implicit trust in the form of Trust B: that is, it is not directly about team members' competence or reliability. The ease with which our questions about trust were answered suggests that there is a form of trust that is tacit though fairly easily available for reflection. In day-to-day clinical practice, these experts are not explicitly or directly concerned with who they trust and why. When they do reflect on trust, what they tend to talk about is how they assess evidence, not people. The reasons for trusting are not consciously or explicitly reflected upon when collaborating. Instead, team members attend to the task at hand and reflect on how it is executed and what are the standards for a good completion of the task. Several of the quotes also mentioned the interactions between people with different expertise, through which the way they accomplish their tasks are calibrated to each other. This is an important aspect of the intersubjective framework for trust, or the 'space of reasons'. In the next section, we will expand on how this is established. 
That trust practices are implicit does not mean that trust is blind. Most authors agree that it takes time and experience for a person to become acquainted with another individual and trust is something that builds up gradually during that time, in a process that can be more reflective then implicit (Baier 1986; Jones 1996; Origgi 2004; Pettit 1995). Susann Wagenknecht (2014) has studied the strategies of scientists in interdisciplinary research teams to handle epistemic dependence in practices of incomplete trust. For example, through dialoguing practices and explanatory responsiveness (being able to address the listener's epistemic needs) researchers can probe the competence of the others and by asking them to explain something, can assess whether the explanation makes sense on a 'meta-disiciplinary' level (i.e., the logic of the other's reasoning). In addition, researchers can assess impersonal characteristics, such as track record and training to evaluate the other's trustworthiness (Wagenknecht 2014). Hence, trust is placed in the other on the basis of prior experiences and critical reflection on those experiences and the other's competence, as the consultant clinician in Quote 6 also contends.

While it is important to note that implicit trust is not blind trust, it is just as important to note that its sight is not simply given, and that it has to learn to see: that is, it has to learn what are possible reasons for trusting the expertise of others, since these are, by definition, not within the purview of everyone in the team. We claim that what counts as reasons for trust in the expertise and competence of others is established indirectly, while attending to other things besides trust. Therefore, it is worth looking at what members of the teams with different expertises do attend to. In the MDT meetings that we observed, and in several of the interviews, a main preoccupation was frequently that there is convergence of different views. This arises in different ways. We will give a concrete example of such convergence regarding imaging in the next paragraph (Paragraph 6.4.), but first we will lay out how convergence operates in weekly MDT meetings.

As we have noted, in our PH case study, the clinicians and radiologists conferred in weekly radiology MDT meetings. In these meetings, generally the consultant opens with introducing the patient by giving a summary of the previous course of the disease, the clinical signs and symptoms, other test results and the specific queries that they have. Then, the radiologist presents the images, shows specific findings and compares them with images from different modalities and with earlier images if available and sometimes asks for clarification about the patient's clinical history from the clinician to refine their evaluation. After what can be quite protracted discussion and going back and forth between images, and sometimes metrics, the consultant concludes the interaction by making a note of the shared conclusion and the follow-up plan for that patient. This is spoken out loud while 
the consultant is writing it down in the patient's clinical record. This allows for a shared 'ownership' of the decision.

In these types of repeated interactions, medical teams cultivate a collection of stable, agreed upon orientations towards evidence and knowledge that builds up an intersubjective framework within which claims and interpretations can be justified, and decisions can be arrived at and shared by others. Face-to-face meetings, such as these MDT meetings play an important role in the establishment of these shared frameworks. They provide a place where orientations towards evidence are coordinated and calibrated, where interpretations can be shared and explained and where experts learn from each other, developing a shared basis for diagnosis and treatment of the population of patients that they specifically manage collaboratively. In this process, pulmonary consultants and radiologists learn from each other and come to interpret evidence such as imaging and the clinical background in a similar way as their colleagues with a different background, as the pulmonary consultants express in Quote 3 and 5 and a radiologist in Quote 4.

An important aspect of this process is the validation of measurements, imaging and interpretations for the local situation (the clinical team as well as patient population, see Quote 9 from a consultant pulmonary clinician) and the calibration of different types of evidence provided and obtained by different experts. For example, after voicing the team's decision, the consultant clinician also mentions the right heart catheter measurements, which allows for a final integration of all evidence and helps radiologists to get a feel for the correlation between the imaging and right heart catheter findings which are considered to be the gold standard measurement to diagnose $\mathrm{PH}$. 


\section{Quote 9}

Interviewer: "so, and this validation, is it about... correlating it to other information, or is it also about... learning the interpret the images?"

P: "I think it's both. Because if somebody showed me a paper today that said, you know, it proved from a group of... a hundred pulmonary hypertension patients that a MR cardiac output and estimates of certain parameters were just as good as the right heart catheter... then I'd say that's very good, but I'd want to see, you kind of also want to see it in other centres, and in your own patient population. And, you'd still want to build up your own experience to feel that that was correct within your unit [...] So if you got a paper from a.. an eminent... unit in Amsterdam, or somewhere, saying how fantastic MRI scan is. That may work perfectly well for them... but will it work for you? With the department you have, with the scanner you have and with the staff you have processing and reporting your scans. Probably, because they're very good here. But you don't actually know that until you see it for yourself, do you? So there's whole different levels of validation." (P10, consultant pulmonary clinician)

In short, by correlating and calibrating findings from different sources as well as interpretations by different experts in repeated interactions, such as MDT meetings, the medical team develops an inter-subjective understanding of what counts as evidence, how different pieces of evidence should be handled and fitted together to produce a 'picture' of a patient and make diagnosis and treatment decisions. Instead of reflecting on who they trust and for what reasons, what the team devotes a great deal of energy to is building an intersubjective framework where what counts as a 'good report' or a 'good question' or a 'good decision' is given shape. It is only within that inter-subjective framework that what counts as a reason for trust can meaningfully be reflected upon when it is actually reflected upon. This intersubjective framework in which the different experts come to have shared orientations, a shared way of seeing (in the perceptual and conceptual senses) provides something that team members can look at and consider, when they are considering whether they trust the outputs of the expertise of others. We call this an intersubjective space of reasons, where members share similar orientations to what counts as a reason or justification for interpretations, judgements or decisions, and for the trustworthiness of experts with whom researchers are in a relationship of epistemic dependence.

So far we have considered the relationship between radiologists and consultants, which by its nature, is geared towards the images used for diagnosis and treatment decisions. With our next example, we dig further down in the relationships around an imaging intensive 
team, which not only uses images but also researches and develops new imaging tools and techniques. Therefore, apart from the clinical team with its relationships extending toward staff and patients, there is also, on the other side, its relationships extending to the radiography department of the hospital, and the imaging engineers, computer scientists and others.

\subsection{Imaging mediates trust practices in clinical decision-making: developing common ways of seeing}

One way of establishing an intersubjective space of reasons - what counts as something to reflect on and what might be a reason to explicitly trust or mistrust - is through the establishment of what is worth looking at.

The PH team in our case study makes use of an MRI scan called cardiac magnetic resonance imaging (CMRI) to assess the anatomy and function of the cardiac chambers. This specific MRI sequence synchronises a person's heart rhythm with MRI data, resulting in a reconstruction of the cardiac cycle that resembles a beating heart. These images are used to assess the function of the right heart for prognosis and disease severity, and of the left heart to exclude left heart disease, by visual assessment of chamber anatomy, contraction and potentially leaking heart valves.

CMRI imaging is a recent development in $\mathrm{PH}$ diagnosis and within the $\mathrm{PH}$ team, the clinicians, radiologists and radiographer had to establish together what is worth looking at in these images. For example, they have to agree that the right ventricle function and morphology are relevant signs of the disease progression, that CMRI can be used to assess the right ventricle function and morphology and, more basically, which part of the image refers to the right heart. Through a process of tinkering with the image acquisition protocol and processing, image interpretation, calibration with clinical outcome by radiographers, radiologists and clinicians, and an ongoing dialogue about which images are most useful, the attention of the whole team is directed at those aspects of the images that they come to agree are worth looking at. Going deeper than the corresponding interpretations and orientations to reasons that we discussed in the previous section, this process around the development of new imaging protocols allows for an alignment of vision that underlies those interpretations and reasons. Alignment at this level produces shared modes of looking at the images, or common modes of perception, the 'sight styles' that we have already mentioned in Paragraph 6.1. When these are brought into play, imaging shifts from its role as being what expertise is about - discussed in Paragraph 6.3. - to playing an 
important role in bringing about alignment at this rather deep level, that makes possible the 'space of reasons' within which trust practices can take hold.
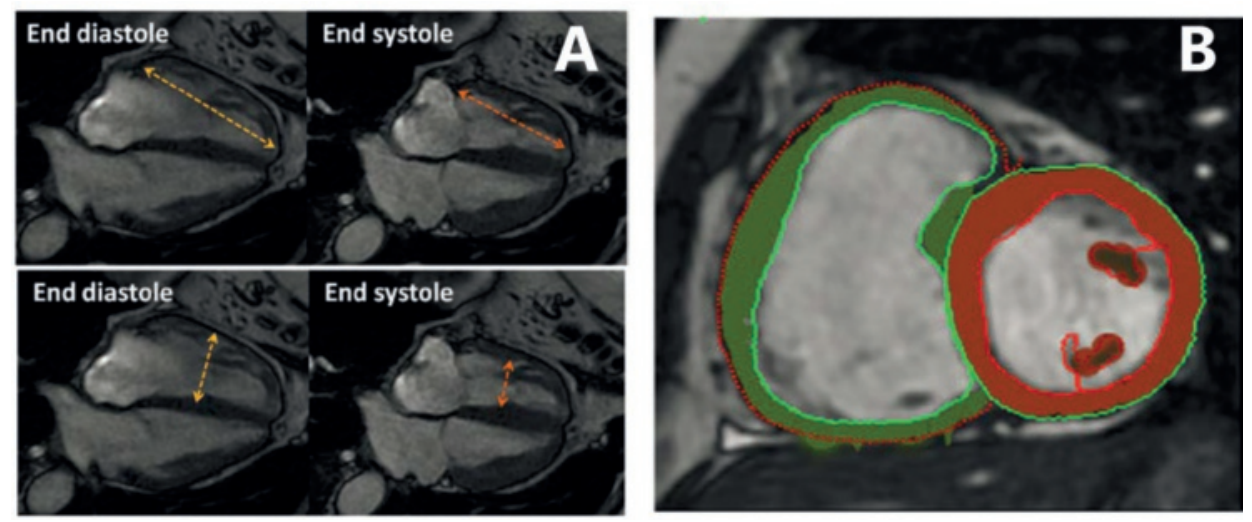

Figure 6.1: Drawing contours to measure the right ventricle ejection fraction (RVEJ)

(A) Cross section of the heart, that provides a "four chamber" view: all four chambers of the heart are visible. The volume of the right ventricle (upper right chamber) is measured at two moments in the cardiac cycle: immediately before contraction (the end-diastolic volume, left) and immediately after contraction (the end-systolic volume, right). (B) An example of the drawing of a contour of the right and left ventricle wall. Source: Rajaram (2013)

To illustrate this point, we discuss an example in more detail: that is, the drawing of the contour of the right heart ventricle (see Figure 6.1). This example is drawn from another central relationship for this team of highly image oriented medical practitioners, that is, the relationship between radiologists and radiographers, who produce the images and process them in the first instance.

In addition to visual assessment, the CMRI images are also processed to quantify predetermined parameters such as the right ventricular ejection faction (RVEJ), a measure held to be clinically important by correlating with disease severity and prognosis. To measure RVEJ, the volume of the right ventricle is measured at two moments in the cardiac cycle: immediately before contraction (the end-diastolic volume) and immediately after contraction (the end-systolic volume), by drawing the contour of the right ventricle in all slices covering the right ventricle volume for the two points in the cardiac cycle. A radiographer draws the right ventricle contours after which a software program calculates the ejection fraction and other metrics characterizing the right ventricle function that are summarized in a report containing numbers and diagrams which the radiologists receive in PACS.

The production of this contour of the right ventricle and its associated metrics is another illustration of distributed expertise, since the radiologists have to trust the radiographers' 
skill in producing and processing the raw data of the images. Team members have to agree that defining the end-systolic and end-diastolic volumes are good ways to determine RV ejection fraction and that this metric is clinically relevant. Radiographers draw the contour of the right ventricle, defining which part of the image refers to the ventricle wall. In this way, they are making a knowledge claim, which radiologists need to be able to trust. In iterative interactions with radiologists and engineers, the 'right' way of drawing the contour so that the appropriate metrics can be derived from it, is determined. Together they bring to the fore this contour, and define how it needs to be. Because several people have been involved in the development of this metric, there can be discussions regarding how an image looks, to the eye, and the metrics. These discussions around weighing up qualitative and quantitative features of images occur frequently, Quote 6 being but one example which acknowledges how the 'numbers can be wrong' and can be inconsistent with how something 'looks'. That is, these specific features about the images are what are focused upon, in the production and interpretation of the images, and all produce a discourse of reasons around the images in terms of which expertise of people, but also the images themselves, are taken to be trustworthy or not. All these things have been established through a long history of interacting and collaborating, aligning their way of looking at CMRI images in the identification of the right ventricle wall and calibrating their interpretation of the metric with clinical outcome. Indeed, as one of our participants noted, skills for older technologies are displaced in the development of skills needed for newer technologies, as they are no longer practiced (see Quote 10 from a consultant cardiothoracic radiologist).

\section{Quote 10}

P: "before, we used to use, just contrast angiography, so what we used to do was, to look at the pulmonary arteries, we used to just inject contrast and then just look at the flow of the contrast in the pulmonary artery. So very... it's an invasive procedure, so you have to have a catheter put in the groin and... but, that's more or less obsolete these days. So we don't, we hardly do one a year.

[...]

Interviewer: "do you miss anything about imaging modalities that you, you know... is there any time that you would say, well we could have seen that on [another older imaging modality]..."

P: "the thing is, because we don't do it that often, we're losing the skill to interpret the... [...] So, you know, if somebody gives us a pulmonary angiography now, I think we'll all struggle to identify what's happening." (P4, consultant cardiothoracic radiologist) 
At this deep level of engendering ways of looking, medical images play an important role in assemblages of distributed knowing. Images fulfil this role in two ways, firstly as epistemic objects that can be distributed among all members of a team, as well as interpreted and discussed, thus facilitating communication and sharing of information and thus mediating the establishment of a space of reasons for trust and distrust. Images allow different experts to converge on their interpretation of a case by being an object to refer to, to relate their own and other's interpretation as well as other evidence from the case to. As shared objects that anchor shared interpretations, images are often regarded as more objective than some other types of evidence, such as clinical history or physical examinations, as pointed out by the consultant clinician in Quote $11 .^{49}$

\section{Quote 11}

Interviewer: "An image... is seen as more objective than..."

P: "It's more objective. The other thing is that you can see the structure in front of you. The structure for example of the heart, and the function of the heart. So you can't doubt that, if you have a heart that's not functioning well, it's pretty obvious. Not always, but many times." (P5, consultant cardiologist)

Secondly, the sharing of images and communicating through them produces shared vision through which the members of the assemblage come to see and perceive in a common way. According to Goodwin (1994), "the ability to see relevant entities is not lodged in the individual mind, but instead within a community of competent practitioners," (Goodwin 1994, p. 626). In medical teams, the ability to see relevant entities is developed in interactions between clinicians, who know and can relate to the clinical case, radiologists who interpret the images in terms of the disease as well as technological limitations and radiographers who are aware of the actual production of images. In these interactions they establish what can be seen on the images and how this relates to clinical outcome. Developing shared vision like this, the medical team develops an even stronger basis for

49 It might be objected that the increasing use of images is a sign of reducing the need to trust others, and indeed, even oneself. Images, and in particular, the quantification that is associated with many types of images, is often rhetorically associated with greater objectivity (Joyce 2008). However, this is not really borne out by fieldwork, including our own, since how the imaging modalities come to be used in specific contexts, how they are taken and processed, involves skills and expertise at every step, and therefore precisely the kind of teamwork that we have been describing here 
sharing interpretations, and building up a shared orientation towards what counts as reasons for accepting knowledge claims of others.

A shared way of seeing coevolves with the development of new imaging modalities. In our field study, the physicists, radiographers and radiologists involved in $\mathrm{PH}$ imaging have a long history of collaboration, developing methods to analyse and evaluate CMR images and metrics together. The technologies, e.g., the scanner, the sequences facilitating the acquisition of CMR images, the image processing algorithms and the software tools that enables drawing the right heart ventricle contours, calculating the ejection fraction and sharing the results, play a crucial and active role in these processes. The technologies, the users, the ways of looking, and the possible knowledge claims coevolve with each other. MRI, by producing a specific type of contrast, between soft tissues, drives a specific kind of visualisation of the heart muscle, and the method of ECG-gating allows visualization of movements of the heart during a complete heart cycle, enabling CMRI. Clinicians and radiologists involved in diagnosis and treatment of $\mathrm{PH}$, from being familiar with heart anatomy and physiology, recognise the relevant structures (i.e., septum, ventricles and valves), and from being familiar with what type of information is required in clinical practice, they recognise which relevant questions might possibly be answered by these types of imaging. However, they need to learn how to recognise deficiencies and how to evaluate function by relating images to clinical outcomes. Together with an ongoing and rigorous discussion, these interactions between radiographers, radiologists and clinicians, and the imaging technologies pushes the development and tweaking of acquisition sequences to improve image contrast for those specific practices, and image processing and analysis algorithms to produce relevant metrics such as right heart ventricle ejection fraction. Such developments further reinforce the trust framework within which medical decision-making operates.

\subsection{Discussion}

Social epistemology addresses knowing in terms of social interactions, such as team collaborations, or social environments, such as institutions or scientific communities. Focusing on social aspects of knowing implies that attention must be paid to epistemic dependence and trust. In this paper we have examined how trust operates in an image intensive clinical setting. Our goal was to uncover the 'mechanisms' of implicit trust in a complex epistemic system where team members with different roles and expertises have to collaborate to formulate shared diagnosis and treatment decisions and imaging technologies play an important role. Elsewhere, we have studied the socio-technological 
epistemology of clinical decision-making and image interpretation as a social and collective activity (van Baalen and Carusi et al. 2016). By studying how implicit trust operates in a clinical team, we have elaborated further on these practices

Figure 6.2 illustrates the different forms of trust that we take to be at play in clinical decision-making teams. Trust A is trust that is given without awareness or reflection on reasons (although there may be reasons), whereas in Trust B there is reflection, but this is directly on accomplishing a joint task; in the process of accomplishing the joint task, reasons for assessing the task indirectly also become reasons for trusting (or not) team members. Without these reasons that are jointly 'owned' it is difficult to see what trusting the expertise of another (whose expertise one precisely does not share) would actually consist in.

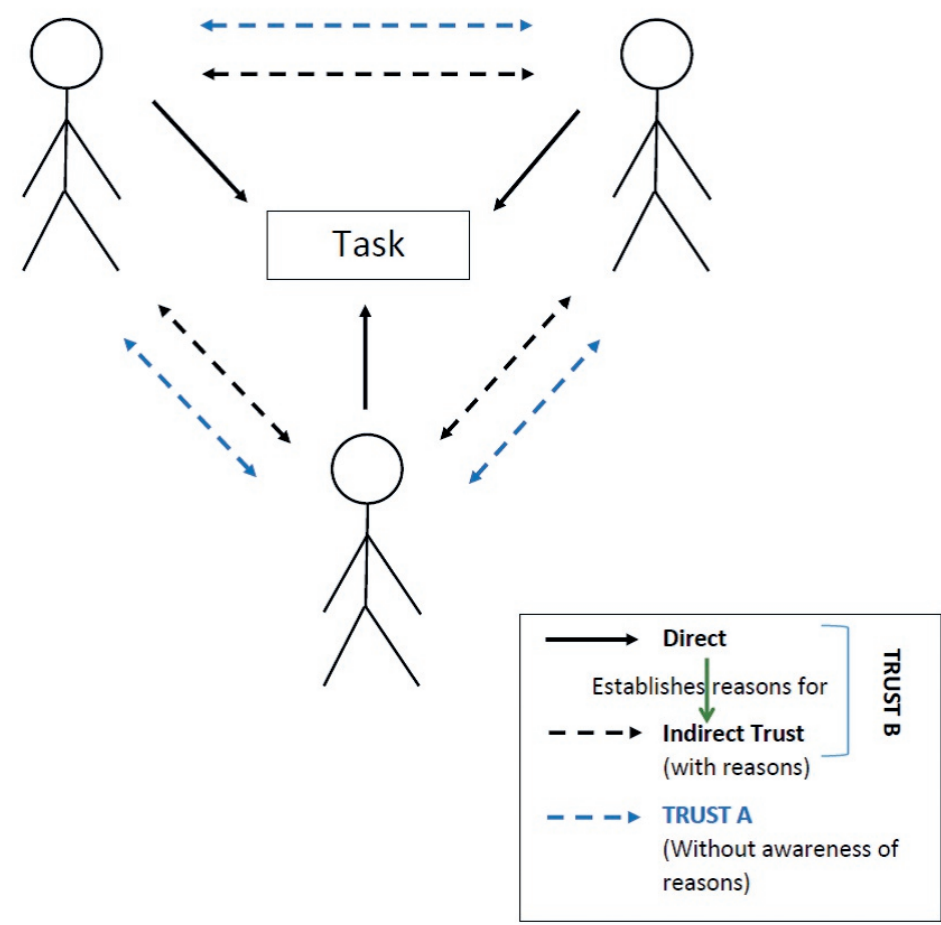

Figure 6.2: Diagram of the different forms of trust at play in clinical decision-making teams. 
Through our fieldwork and conceptual analysis we have identified several characteristics of trust practices: trust practices a) are indirect, in that they do not directly evaluate the level or kind of expertise of the trustee; b) are implicit in that they do not reflect on reasons for trust in advance, or there may well not be reasons in advance; c) are interactional and highly contextualised in joint tasks; d) require labour from both the truster and the trustee, and a reversibility between them (the truster is also the trustee and vice versa); e) iterative, they develop and evolve over time; f) operate by building up a common stock of appropriate reasons for accepting evidence or information or not that truster and trustee both share and both have access to. The common stock of appropriate reasons for accepting evidence or information is also a common style or way of assessing evidence or information, and is built up through forging common modes of perception, 'sight styles' in which technologies play a mediating role. Through these means the expertises of the others are less opaque, more accessible and comprehensible for collaborators with another specialty, and can be brought to awareness and reflected upon if needs be in ways that are meaningful across the different expertises. Our example of the measurement of an MRI metric shows that there is also an overlapping of reasons for trusting different things or different entities: reasons for trusting an image or a number overlap with trusting the expertise of a person who is involved with producing or interpreting that image or number. We would go further and claim that trusting the expertise of someone else on whom one is epistemically dependent is rarely directly aimed at that person's expertise, but is instead nested into ways of trusting evidence and information that are engendered through iterated interactions, which bring collaborators into a common space of reasons. Quote 8 expresses this well: "I mean we founded our collaboration on a need to get something going, and that's always a good starting point. You work on something and you get it working and you see that it's actually been useful". In this quote, we see the social bond of 'getting something going', and in the other by the same participant, Quote 7, we see the dependence of epistemic trust on other forms of trust: moral and interpersonal, attested by a positive evaluation of the other's character ("he's a pragmatist, he's a people's person") a common commitment to shared norms and values ("his main priority is patient care", "non-political") and pragmatic ends ("a need to get something going").

We turn now to a brief consideration of two further challenges that arise from our account of implicit trust in multidisciplinary clinical settings. Even though trust is implicit, reasons for trusting can come to be attended to and reflected upon; but these reasons are not pre-given. It is only through participating in the creation of an intersubjectively shared framework for assessing evidence and information, that reasons for trusting or distrusting emerge. In other words, we claim that trust and reasons for trust are interwoven and develop in the same situation of epistemic dependence in collaboratively completing a 
task, while team members attend to the task at hand and criteria for the execution of that task. As we have argued, establishing and cultivating a shared space of reasons requires agreement about what is worth looking at, what specific pieces of evidence say about specific patients, and in the clinical team we observed this agreement was usually reached through deliberation and consensus. However, as some of the participants in our field study and several authors (e.g., see: Esser 1998, Solomon 2006, Urfalino 2014) have pointed out, this is not without its problems.

Groupthink, group dynamics and social pressure may impede the quality of a group decision reached through deliberation and consensus for several reasons. Consensus agreement does not necessarily reflect the opinions or input of all the members of a group equally, because rather than a summation of all opinions, consensus is the conclusion that no one in the group objects to (Urfalino, 2014). In addition, usually one team member has the responsibility to summarise all inputs into a group decision, which involves weighting all inputs. In that process, the input from people with more authority or a stronger voice can be given more weight hence have more influence on the resulting group decision. Additionally, since in these meetings consensus is the desired outcome, minority opinions may feel pressured not to voice their opinion, thus consensus may discourage disagreement (Solomon 2006). Dissenting individuals may feel pressured to change their mind, or not share knowledge of contrary evidence, therefore, deliberation and consensus may not be the best way to collect and fit together all available knowledge or opinions. We have not assessed whether groupthink and other social dynamics associated with consensus affected the decision-making processes in our fieldwork.

However, this leads to a deeper issue, that has potentially serious implications. It is difficult to see from which perspective or foothold an evaluation of the practices and intersubjective framework is possible. For example, the development and implementation of a new technology or technique requires a great investment of time and practice to develop the shared way of seeing that is necessary for it to operate well in a context geared towards providing reasons for clinical decision-making. As we have noted (in Paragraph 6.4.), in the development of the skills needed for the new technique, other techniques could become obsolete because the skills for using them are no longer practiced. In this case, at least one of the bases for external comparisons and evaluations of the practices and intersubjective framework in operation in a team, can be eroded. This can lead to skewed practices, that could be either not beneficial or even harmful to health. ${ }^{50}$ How this is balanced against 
other bases for comparison and evaluation is something we have not broached in this article. We will try, however, to give an outline of how both of these challenges, that of groupthink/dynamics and that of independent evaluation, might be broached.

To return to a distinction mentioned in the introduction, the account that we are offering does not fall into either reductionist nor anti-reductionist epistemologies of trust. It is a holist account of trust, that stresses the interdependencies between different kinds of trust. It might be thought of as a circular mode of trust, and this is the underlying worry of both the challenges we have mentioned. There is certainly bootstrapping in the account of trust we are advocating, but it is not necessarily vicious or undermining of trust. Moral or interpersonal trust in the character and professionalism of others does a lot to lay the ground for epistemic trust in the expertise and skills of others, in specific instances when the diagnosis and treatment of specific patients is at issue; implicit epistemic trust might bootstrap on moral or interpersonal trust, sufficiently to 'get things going'. The intersubjective space of reasons could well give rise to a form of 'groupthink' and enclosed local practices: this is a real and not only hypothetical possibility. However, to draw from this the normative lesson that this form of trust is weak or unreliable would be incorrect. To insist on 'independent' reasons to remedy this potential 'groupthink' is not helpful because there may not be ways of actually applying these independent reasons meaningfully within the team. Meaningful reasons are criteria that are shared by teams. The remedy instead is to look at the broader settings in which clinical teams are embedded. Clinical teams are highly porous entities, and not at all self-enclosed. They can subsist for quite some time with changing members; new members bring in their background education and experience; there are often visitors and others who attend MDTs; they operate in hospitals with organisational structures that link them to other hospitals and institutions, with mechanisms of oversight; many members of the team also undertake research and publish and are therefore also subjected to the scrutiny of peer reviewers. Thus, shared by the team does not imply closed off within the team, impervious to scrutiny. It is these overlapping contexts of institutions of trust that result in the holist form of trust we have been describing not being necessarily subject to vicious circularity. Instead, it is a web of interconnected forms of trust that are made more robust through their interconnection. In order to address the 'bootstrapping' problem, we need to be able to view practices from different distances and perspectives: from the close granular view of how teams with distributions of expertise across members operate, medium-scale views of how they are embedded within clinics and institutions; and larger more distant views of how they operate across clinics and institutions. The normative implications are that the justification for trust practices need to be sought in these interconnections between establishing what can count as reasons for trusting expertise, and the intersecting contexts in which reasons 
are produced, communicated and exchanged from within teams, to across institutions. An applied philosophy of medicine could do valuable work in shedding light on the nature of these interconnections and how they contribute to the robustness of trust practices.

In conclusion, a social epistemology of clinical decision-making involves directing attention to epistemic dependence between team members with different expertises and roles in the clinic. Epistemic dependence, in turn, requires trust. In this paper we have given an account of the trust implied in epistemic dependence as a form of implicit trust in these teams, that has both epistemic and moral elements. Through implicit trust, teams build an intersubjective framework, in which reasons for trusting the expertise of others on whom each is dependent are very closely intertwined with reasons relating to identifying and assessing evidence. It is only within such a common framework that trusting or mistrusting become meaningful in these contexts. Trusting well in clinical decision-making teams is what one does while attending to other things than expertise, most importantly, agreeing on what are good reasons for recognising a task well done. 


\section{References}

Adler, J. E. (1994), 'Testimony, Trust, Knowing', Journal of Philosophy, Inc., 91 (5), 264-75 DOI: 10.2307/2940754. Andersen, H. and Wagenknecht, S. (2013), 'Epistemic dependence in interdisciplinary groups', Synthese, 190 (11), 1881-98 DOI: 10.1007/s11229-012-0172-1.

Baier, A. (1986), 'Trust and Antitrust', Ethics, 96 (2), 231-60

Brandom, R. (1995), 'Knowledge and the Social Articulation of the Space of Reasons', Philosophy and Phenomenlogical Research, 55 (4)

Carusi, Annamaria (2008), 'Scientific visualisations and aesthetic grounds for trust', Ethics and Information Technology, 10 (4), 243-54 DOI: 10.1007/s10676-008-9159-5.

Carusi, Annamaria (2009), 'Implicit Trust in the Space of Reasons and Implications for Technology Design: A Response to Justine Pila', Social Epistemology, 23 (1), 25-43 DOI: 10.1080/02691720902741423.

Esser, J.K. (1998), 'Alive and well after 25 years: A review of groupthink research', Organizational behavior and human decision processes, 73 (2), 116-41 DOI: 10.1006/obhd.1998.2758.

Faulkner, P. (2007), 'On Telling and Trusting', Mind, 116 (464), 875-902 DOI: 10.1093/mind/fzm875.

Fleck, L. (1979), Genesis and development of a scientific fact (Chicago: University of Chicago Press) ISBN: 9780226253251.

Fleck, L. (1986e [1947]), 'To look. To see. To know.', in R.S. Cohenand and T. Schnelle (eds.), Cognition and fact. Materials on Ludwik Fleck (Dordrecht: Reidel Publishin) ISBN: 978-94-009-4498-5

Friedrich, K. (2010), “Sehkollektiv’: Sight Styles in Diagnostic Computed Tomography', Medicine Studies, 2 (3), 185-95 DOI: 10.1007/s12376-010-0050-4.

Frost-Arnold, K (2014), 'The cognitive attitude of rational trust', Synthese, 191, 1957-74 DOI: 10.1007/s11229012-0151-6.

Goodwin, C. (1994), 'Professional Vision', American Anthropologist, 96 (3), 606-33

Hardwig, J. (1985), 'Epistemic dependence', The Journal of Philosophy, 82 (7), 335-49 DOI: 10.2307/2026523

Hardwig, J. (1991), 'The role of trust in knowledge', The Journal of Philosophy, 88 (12), 698-708 DOI: $10.2307 / 2027007$

Hertzberg, Lars (1988), 'On the attitude of trust', Inquiry, 31 (3), 307 - 22

Jirotka, M. et al. (2005), 'Collaboration and Trust in Healthcare Innovation: The eDiaMoND Case Study', Computer Supported Cooperative Work (CSCW), 14 (4), 369-98 DOI: 10.1007/s10606-005-9001-0.

Jones, K. (1996), 'Trust as an Affective Attitude', Ethics, 107 (1), 4-25

Joyce, K. (2008), Magnetic Appeal: MRI and the Myth of Transparency. (Ithaca and London: Cornell University Press) ISBN: 9780801474569.

Kappel, K. (2013), 'Believing on trust', Synthese, 191 (9), 2009-28 DOI: 10.1007/s11229-013-0376-z.

Lackey, J. (2010), 'Testimony: acquiring knowledge from others', in A.I. Goldman and D. Whitcomb (eds.), Social Epistemology: Essential Readings (Oxford University Press) ISBN: 978-0195334616

Lagerspetz, Olli (1998), Trust: The tacit demand (1: Springer Science \& Business Media) ISBN: 0792348745. Lagerspetz, Olli (2015), Trust, Ethics and Human Reason (Bloomsbury Publishing) ISBN: 1441146091.

Lahno, B. (2001), 'On the Emotional Character of Trust', Ethical Theory and Moral Practice, 4 (2), 171-89 DOI: 10.1023/a:1011425102875.

Lewis, J.D. and Weigert, A. (1985), 'Trust as a Social Reality', Social Forces, 63 (4), 967-84 DOI: 10.2307/2578601. McCraw, Benjamin W. (2015), 'The Nature of Epistemic Trust', Social Epistemology, 29 (4), 413-30 DOI: 10.1080/02691728.2014.971907.

Mollering, G (2001), 'The Nature of Trust: From Georg Simmel to a Theory of Expectation, Interpretation and Suspension', Sociology, 35 (2), 403-20 DOI: 10.1177/S0038038501000190. 
Oakley, J. and Cocking, D. (2001), Virtue Ethics and Professional Roles (Cambridge and New York: Cambridge University Press) ISBN: 9780521027298.

Origgi, G. (2004), 'Is trust an epistemological notion?', Episteme, Cambridge University Press (CUP), 1 (1), 61-72 DOI: ijn_00000653.

Pettit, P. (1995), 'The Cunning of Trust', Philosophy \& Public Affairs, 24 (3), 202-25 DOI: 10.1111/j.10884963.1995.tb00029.x.

Rajaram, S. (2013), 'Imaging in pulmonary hypertension: the role of MR and CT', (University of Sheffield).

Shapin, S. (1994), A Social History of Truth: Civility and Science in $17^{\text {th }}$ Century England (Chicago \& London: Universiy of Chicago Press) ISBN: 9780226750194

Solomon, M. (2006), 'Groupthink versus The Wisdom of Crowds: The Social Epistemology of Deliberation and Dissent', The Southern Journal of Philosophy, 44 (S1), 28-42 DOI: 10.1111/j.2041-6962.2006.tb00028.x.

Toulmin, S. (2001), Return to Reason (Cambridge, MA \& London: Harvard University Press) ISBN: 9780674012356.

Urfalino, P. (2014), 'The Rule of Non-Opposition: Opening Up Decision-Making by Consensus', Journal of Political Philosophy, 22 (3), 320-41 DOI: 10.1111/jopp.12037.

van Baalen, S. et al. (2016), 'A social-technological epistemology of clinical decision-making as mediated by imaging', J Eval Clin Pract, DOI: 10.1111/jep.12637.

Wagenknecht, Susann (2014), 'Facing the Incompleteness of Epistemic Trust: Managing Dependence in Scientific Practice', Social Epistemology, 1-25 DOI: 10.1080/02691728.2013.794872.

Weisz, G. (2003), 'The emergence of medical specialization in the nineteenth century.', Bulletin of the History of Medicine, 77 (3, Fall 2003), 536-74 DOI: 10.1353/bhm.2003.0150.

Williams, B (2002), Truth and Truthfulness : an Essay in Genealogy (Princeton \& Oxford: University of Princeton Press) ISBN: 9780691117911.

Wittgenstein, L. (1969), On Certainty (New York: Harper Torchbooks) 


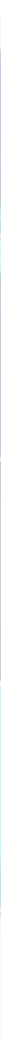


Discussion and conclusions

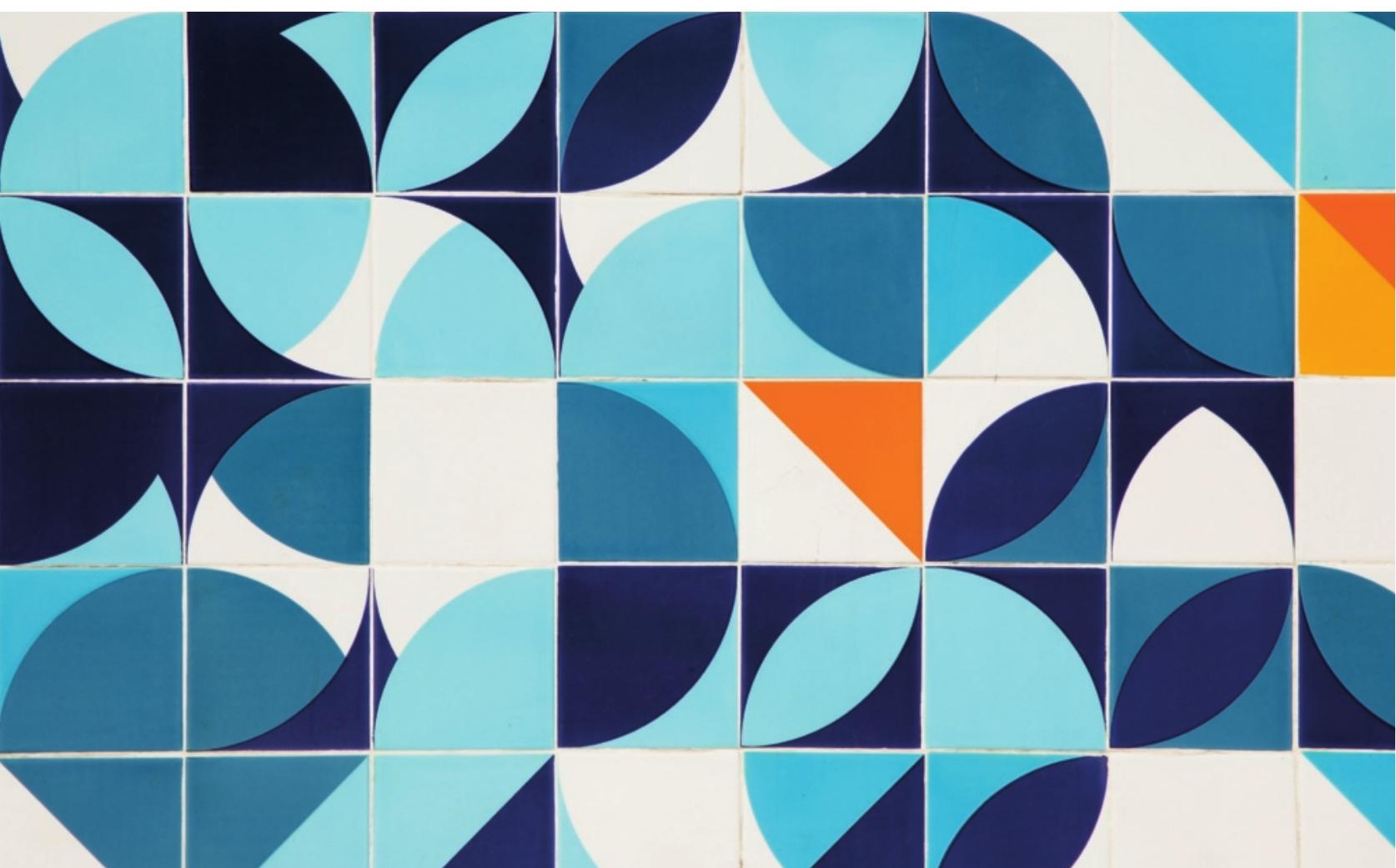




\section{Discussion and conclusions}

In this dissertation, I have focused on knowing in medicine and the role that expertise, imaging technologies and interdisciplinarity play in the construction and use of knowledge in this professional practice. I have turned to medical practice and the professionals who operate within this practice, their goals, their values and their cognitive abilities, to study how knowledge is constructed and used in medical practice. In Chapter 1, I have defended the claim that doctors have epistemological responsibility for their clinical decisions, being responsible for the collection, critical appraisal, interpretation and fitting together of heterogeneous sources of evidence into a 'picture' of the patient. In Chapter 2, I argued that understanding and evaluating medical expertise involves an account of the epistemic activities that clinicians should be able to perform and the cognitive skills that allow them to do this. In Chapter 3, I claimed that experts acquire a disciplinary perspective by being trained within a domain that shapes how they deal with a target system. An interdisciplinary solution is achieved by taking each perspective on the target system and using each of them to reason about a phenomenon of interest studied or dealt with from one disciplinary perspective (i.e., which problem concerning this phenomenon has to be addressed, and how, leading to what solution) and how that affects the phenomenon of interest studied by experts from another disciplinary perspective. In Chapter 4, I argue that, for the development of a new imaging technology for clinical practice, epistemic contributions from future users, the clinicians, is imperative. Technology developers and clinicians enter into a process of interdisciplinary collaborative reasoning to establish what is 'seen' in the image and what it can 'tell' about the patient's condition. In Chapter 5, I argue that knowing in current medical practice has an inherently social character - in the sense of being distributed over professionals with different expertises and roles who collaborate to establish a shared decision and that medical imaging plays a mediating role in these epistemic collaborations. Finally, in Chapter 6, I defend the claim that the collaborative character of epistemic activities in clinical decision-making involves complex social practices of trust. Trust in these practices is implicit, in the sense that trusting the expertise of others occurs while the members of a team focus on other tasks, most importantly, building up an intersubjective framework of common ways of identifying and assessing evidence. It is within this intersubjective framework that trusting or mistrusting becomes meaningful in professional (collaborative) settings. 
In the introduction, I have introduced two more general claims that are developed throughout the dissertation, that bring out new ways of approaching epistemological questions and challenges in practice:

I. Knowers play an active and irreducible role in the construction and use of knowledge in practice.

II. Knowing in real-world practices is inherently collaborative.

These claims refer generally to practical fields in which knowledge is used and constructed to intervene in a real-world situation, such as engineering, education and policy-making. As mentioned earlier, I regard the construction and use of knowledge for and in clinical practice as an interesting case for the philosophy of science in such practical fields. I have argued that in these practices (scientific) knowledge and other sources of information are used to construct new knowledge about the specific situation at hand. In Chapter 1, I have called this a 'picture' of a patient, and in Chapter 2 I have argued that this 'picture' is constructed such that it can be used as an epistemic tool for reasoning about the diagnosis and treatment of the patient. Constructing a 'picture' of a specific situation that can be used as an epistemic tool for reasoning about that situation requires a variety of types of evidence, from general empirical or causal laws to very localised, qualitative and rich descriptions. The epistemic quality of these types of evidence cannot be captured in simple rules, as is presupposed by 'evidence-based' movements such as EBM and by traditional, theory-oriented philosophy of science. Rather, the quality of evidence is assessed by diverse epistemic criteria, not only empirical adequacy, logical coherency and consistency with commonly accepted scientific knowledge, but also relevance and utility to the specific situation. In this account, the role of the knower - the professional who uses scientific knowledge to construct knowledge of a specific situation - takes a prominent place. Usually, philosophers of science seem to fear that a dismissal of the idea of objective, detached knowledge paves the way for subjectivism or relativism. Against this, I have argued that an approach that emphasizes that epistemic agents play a central role in the use and construction of knowledge allows a better evaluation of epistemic products than dogmatic adherence to general concepts such as truth, reality and objectivity. Therefore, I introduce an account in which knowers are responsible for how well they perform epistemic activities, which means that they can be held accountable for acquiring, developing and maintaining competence in cognitive skills, and also that they bear responsibility for the quality of the knowledge and reasoning processes, which I have called 'epistemological responsibility.' 


\section{Expertise}

The subtitle of this dissertation refers to three key concepts around which my analysis of knowing in medical practice revolves: expertise, imaging technologies and interdisciplinarity. In my analysis of expertise, I have turned the focus from what is known to who is the knower and how does he or she comes to know. This turn brings with it specific issues, some of which I have addressed in this dissertation. Firstly, the question of how practitioners gain the ability to use and construct knowledge in practice. In Chapter 2 I have argued that it involves expertise, which includes experience, knowledge and skills. More specifically for this dissertation's theme, it involves cognitive skills that enable knowers to perform specific epistemic activities. Expertise is usually learned within a discipline: experts have expertise in a certain field. As experts gain expertise in a certain field, they also adopt a disciplinary perspective that allows them to perform specific epistemic activities, but which also defines what phenomena are of interest, how those phenomena are studied and modelled, and for what purpose, as well as the epistemic criteria that are used to evaluate results. However, most real-world problems - the problems usually dealt with in professional practices - require input from multiple disciplines and involve a division of epistemic labour. Hence, in professional practices experts with different background expertises and professional roles must collaborate. It is in this sense that knowing in real-world practices has an inherently interdisciplinary character.

\section{Interdisciplinarity}

I have studied two types of real-world interdisciplinary knowledge practices - the development of imaging technology for clinical practice, and clinical decision-making. In Chapters 3 and 4 I illustrate the exchange between experts with different expertises, each making different contributions that suit their disciplinary perspective, building on each other to develop an interdisciplinary solution that is internally coherent as well as consistent with each disciplinary perspective. I claim that such interdisciplinary collaborations are imperative to develop an imaging tool that takes into account how the image can provide evidence for decision-making in the intended clinical practice. Developing a technology that is reliable, relevant, useful and interpretable requires input from the imaging technology developer (e.g., an engineer) and the future user (e.g., a clinician). By an empirical study of a multidisciplinary team, consisting of a clinician, a radiologist, a radiographer, and an engineer collaborating on the development of a new MRI imaging technique for clinical practice, I illustrate that what is 'seen' in an image and what it 
can 'tell' about the patient's conditions is established in an interdisciplinary collaborative reasoning process.

In Chapters 5 and 6, I have analysed decision-making by a multidisciplinary medical expert team involved in diagnosis and treatment of patients with pulmonary hypertension. In this practice, in a similar way as my analysis in Chapter 1, heterogeneous types of information - such as the patient's history, clinical examination, lab tests, images and measurements, awareness of personal and social circumstances, observations of the patient by clinicians on ward rounds and by ward staff, or interactions with family - are fitted in to a 'picture' of the patient. These pieces of evidence are generated and interpreted by different people, with different expertises and professional roles. In that sense, knowledge of a patient is distributed among team members. In this account of distributed knowing in clinical practice, team members are epistemically dependent on each other to gain access to pieces of relevant evidence that they do not usually have from their own disciplinary perspective. In some cases, this requires epistemic trust in other team members. In Chapter 6 I have analysed the relationships between trust and reasons for trust, and show that reasons for trust are not sought or given in advance of a situation of epistemic dependence and that they are implicit in the sense of being contained or nested within other actions that are not directly about trusting another person. Reasons for trust are established while team members are busy on a collaborative task, building up a framework of common ways of identifying and assessing evidence. In other words, trust enables collaborations in multidisciplinary teams, but is also established within those collaborations. To get collaborations off the ground team members assess the trustworthiness of their collaborators on other grounds that fall in the 'moral' domain, for example, the goodwill of others. In that sense, epistemic trust is intertwined with other types of trust in interdisciplinary collaborations.

This collaborative account of knowing in practice brings up several issues when relating it to the responsibility account - which was originally aimed at individual professionals that I present in Chapters 1 and 2. Firstly, I assert that heterogeneous sources of evidence are fitted into a 'picture' of a patient that is used as an epistemic tool for further reasoning. How does this work in multidisciplinary teams? Does each team member develop his or her own picture of a patient that each of them then uses to (individually) reason about the patient before combining these interpretations into a shared decision? Or is the basis for a shared decision a 'picture' of a patient that is developed collaboratively. In my view, the latter is the case. Team members are busy gathering, assessing and interpreting evidence from their own disciplinary perspective or clinical role. These pieces of evidence and their interpretation are coordinated and adapted in relation to each other in an interdisciplinary 
collaborative reasoning process - which usually takes place in multidisciplinary team (MDT) meetings - in such a way that they cohesively fit into a 'picture' of the patient that is consistent with the contributions from all team members. Thus, although team members do not have direct access to every piece of evidence, they each contribute to and have a similar understanding of this shared picture of the patient. It is precisely in this process that team members establish what evidence is worth taking into account, how it is interpreted, how it is integrated into a 'picture' of a patient and what that means in terms of the patient's condition and diagnosis and treatment of the patient. This common way of dealing with evidence - a collection of appropriate reasons for accepting evidence that all team members share and have access to - is established in repeated interactions between team members. This creates an intersubjective framework within which (reasons to) trust and mistrust become meaningful.

Secondly, the account of collaborative knowing that I have proposed seems to be at odds with the epistemological responsibility that I have claimed physicians have: being responsible seems to require that a knower has direct control over the processes with which knowledge is constructed and used. Implicitly trusting, on the other hand, implies leaving certain aspects of knowledge construction in the hands of others without explicit consideration. Although this is potentially a serious issue, what I have aimed to show is that although trust is implicit, it is not blind - reasons for trusting can come to be attended to and reflected on; but these reasons are not pre-given. The reasons for trusting are not about trusting directly, but about the task that team members are involved in collaboratively. Trust operates by building up a collection of appropriate reasons for accepting evidence that all team members share and have access to. It is only through participating in the building of this collection, and thereby in the creation of an intersubjectively shared framework for assessing evidence and information, that reasons for trusting or distrusting emerge. As a result, the expertises of the others are less opaque, more accessible and comprehensible for collaborators with another disciplinary perspective, and can be brought to awareness and reflected upon if needed in ways that are meaningful across the different disciplinary perspectives. All team members contribute to creating this intersubjective framework of reasons to accept evidence or not, and adapt their own contributions and standards for assessing evidence to those of others, making sure that it is fitting with their own disciplinary perspective (i.e., it does not conflict with commonly accepted theories, correct usage of instruments and epistemic standards). In other words, in addition to giving an account of knowing in clinical practice in which collaborative knowing and implicit trust are crucial to how multidisciplinary teams operate, I also aim to give an account of how clinicians take part in interdisciplinary collaborations in an epistemically responsible manner. The shared framework allows team members to evaluate 
and assess the contributions from others in the process of interdisciplinary reasoning while contributing to the intersubjective framework in a way that is consistent with their own disciplinary perspective ensures that collaboratively produced knowledge lives up to epistemological standards established in wider (disciplinary) scientific communities.

\section{Imaging technologies}

The third concept I refer to in the title of this dissertation is imaging technologies. Imaging technologies appear in this dissertation in two ways: as a tool that is under development and the focus of interdisciplinary research collaborations, and as a mediator of interdisciplinary collaborative clinical decision-making. To start with the latter, in the multidisciplinary medical team involved in the diagnosis and treatment of pulmonary hypertension that I have observed, imaging was routinely used for diagnosis and follow up. In Chapter 5 I argue that images play an important role in the interactions between team members, being displayed and referred to during multidisciplinary team meetings. In that way images function as a focal point that facilitates the communication between team members: they can point at the images to illustrate or corroborate their interpretation, and other members can use the same image to check their own interpretation of the same image or of other evidence in relation to this image. This allows members of teams to calibrate different types of evidence provided and obtained by different experts. By repeating such exchanges an intersubjective understanding of what counts as evidence and how different pieces of evidence should be handled and fitted together to produce a 'picture' of a patient is established. In that way, medical images play a crucial, mediating role in the functioning of multidisciplinary medical teams.

In addition, images play a mediating role in trust practices. In Chapter 6 I illustrate how team members collaboratively establish what is worth looking at in new images, by presenting the example of drawing the contour of the right heart ventricle. I argue that through a process of tinkering with the imaging technology and an ongoing dialogue about which images are most useful, the attention of the whole team is directed at those aspects of the images that they come to agree are worth looking at and how this relates to clinical outcome. As a result, the vision of all members is aligned and the team has developed a common 'sight style' which provides an even stronger basis for sharing interpretations and building up a shared orientation towards what counts as reasons for accepting knowledge claims of others - in other words, it contributes to building the intersubjective framework of common ways of identifying and assessing evidence. In short, images mediate interdisciplinary collaborative reasoning processes by facilitating 
exchange, framing the epistemic domain and providing a basis for trust practices by supporting the development of an intersubjective framework.

The other way imaging technology appears in this dissertation is as a tool under development and the focus of interdisciplinary research. In Chapter 4, I show how a team of professionals with different expertises enter into an collaborative interdisciplinary reasoning process to figure out what can be 'seen' in new images - called hyperpolarized gas MRI - and what it can 'tell' about a patient. In that chapter, I also introduce a paradoxical situation: for a new measure to be relevant, it is necessary that it provides information that cannot be obtained otherwise, but for the measure to be reliable it has to be checked against a fixed reference, which cannot be provided by another reliable measure. In other words, a new imaging technology has to be coherent with but also go beyond an established imaging (or other measuring) technology. I refer to Hasok Chang, who argues that it starts with identifying 'fixed points' of reference and a way to measure them, without having to establish an absolute scale. After that, it entails a process of epistemic iterations building towards an increasingly precise and reliable tool.

The clinical team that I studied in Chapters 5 and 6 were also involved in the introduction of new MRI tools for the diagnosis of pulmonary hypertension. In Chapter 5 I refer to the introduction of an MRI perfusion scan in combination with a CT angiogram which ultimately replaced the more invasive pulmonary angiogram. In that chapter, I argue that a new imaging technique runs alongside and piggybacks on an existing one. For a while, both imaging modalities are used in the clinic and evaluated in relation to each other, the existing imaging tool providing a reference for the new one. Over this period of time, as the imaging modality is being developed further by tweaking the technology so that it shows those features that are relevant to answer clinical questions, the ability (the expertise and skills) to interpret and use the images also develops. After a while, the new imaging technology becomes the standard of care, and the expertise and skills required to interpret the existing images get lost. The evidence for defects in the lung, for example, comes to be defines in terms of how these defects are visualized in perfusion MRI instead of in contrast angiography.

In Chapter 6 I present the example of cardiac MRI (CMRI) in which I illustrate how radiographers, in collaboration with radiologists and clinicians, define the contour or the right heart ventricle in order to calculate a metric called the right ventricle ejection fraction (RVEJ). I use this example, to illustrate how the technologies, the users, the ways of looking, and the possible interpretations coevolve with each other. MRI produces a specific type of image contrast that enables radiologists to visualise soft tissues and drives a specific 
kind of visualisation of the heart muscle. The method of ECG-gating allows visualization of movements of the heart during a complete heart cycle thereby enabling CMRI. Clinicians and radiologists involved in diagnosis and treatment of patients with pulmonary hypertension $(\mathrm{PH})$ are familiar with heart anatomy and physiology and can therefore recognise the relevant structures (i.e., septum, ventricles and valves) in these image. In addition, they are familiar with what type of information they require in clinical practice and therefore recognise which relevant questions might possibly be answered by CMRI. However, clinicians and radiologists also need to learn how to evaluate the function of the right heart as constituted by the CMRI and the metric (RVEJ), which is obtained by drawing the contour of the right ventricle. Learning this is achieved by relating images to specific patients and their clinical outcomes. Together with an ongoing and rigorous discussion, these interactions between radiographers, radiologists and clinicians, on the one hand, and the imaging technology on the other, push the development and refinement of the technology, thereby improving the information that can be obtained for diagnosis and treatment decisions in the $\mathrm{PH}$ practice. In this case of developing and introducing a new imaging technology, the interdependence between the development of the imaging technique and how users learn to interpret it is illustrated. This is an example of the process of empirical iteration that Hasok Chang has in mind for the development of new measuring tools. In addition, it is an example of how, in practice, professionals deal with the paradoxical situation pointed out above, and manage to establish what information can be derived from new images.

In short, the imaging technology, the interpretations, and the expertise and skills required to effectively use technology coevolve with each other in interdisciplinary collaborations between team members with different expertises over lengthy periods of time. In the process, skills to use other techniques that initially provided a reference point become obsolete over time. This raises the question of how the use of an imaging tool by a particular clinical team - after its use has been established in that team and has taken over the use of other (imaging) technologies - can be objectively evaluated. As I have argued, the expertise and skills required to use the images are developed within the team and therefore, it is seems unfeasible to evaluate the epistemic value and use of an imaging technology from an external point of view. So the interdisciplinary collaborative reasoning process by which new medical imaging technologies are developed and implemented seems to imply circularity as regard to evidence for the value of the tool in practice. Evaluating the value of the imaging tool requires expertise and skills that can only be developed within a multidisciplinary team and by using the tool in practice, for which the value of imaging tool still needs to established. However, the fact that expertise and skills are developed within a team does not mean that it is closed off from external epistemic criteria. Rather, 
the experts involved in the development and introduction into clinical practice of the technology, are experts in a certain domain and therefore have a knowledge and skill base that is shared by a wider scientific community that has commonly accepted methods and epistemic values. Coming to agree on what is worth looking at in an image or how that can be interpreted in relation to a patient's condition is not solely an internal or social process of the team. Rather, during the process each expert assesses whether the interpretation is consistent with methodology, theories or epistemic values from their own scientific community. This safeguards the process of imaging technology development from circularity, which again builds on the notion of epistemological responsibility. In the discussion of Chapter 6 a similar point is made about trust practices, arguing that although meaningful reasons for trust may not exist independent from the team, this does not have to imply circularity or vicious bootstrapping because a medical team is not a closed-off entity and reasons are communicated across other medical and scientific institutions. In this dissertation, I aimed to provide a fine-grained epistemological analysis of interdisciplinary collaborations of a multidisciplinary team aiming at the development of new imaging tool for clinical practice. A better understanding of the epistemological processes by which medical teams are open to external epistemic criteria would also require zooming out, performing (applied philosophical) analysis of the institutional and scientific community-wide processes by which reasons, expertise, skills and knowledge are shared, adapted and established. However, this analysis falls outside of the scope of this dissertation and would be an interesting topic for follow-up research. 


\section{Methodology}

Finally, I want to address the methodology of this dissertation. In the introduction, I claim that one goal of the analyses in this dissertation is to provide a vocabulary to knowers (experts) involved in (interdisciplinary) reasoning processes, in order for them to discuss, explain and justify their epistemic activities. Therefore, I have analysed several real-life practices of clinical-decision-making and imaging technology development. I have performed a qualitative study of clinical decision-making within a medical expert team involved in the diagnosis and treatment of patients with pulmonary hypertension (PH), a rare disease requiring multidisciplinary team involvement. Within this field study, I conducted observations, interviews, video tasks and a panel discussion. In addition, I have videotaped a collaborative task of a multidisciplinary team working on the development of a new magnetic resonance imaging (MRI) tool for the diagnosis of airways diseases such as asthma and chronic obstructive pulmonary disease (COPD), called 'hyperpolarized gas MRI' (HP-MRI). Furthermore, one of the case studies in this dissertation was based on my own experience from being involved in the development of an imaging technology for clinical practice. The methodological approach to analysing empirical materials (e.g., real-world case studies) in philosophy has not been crystallized (yet), and philosophers deal with many different types of empirical materials in many different ways (from rigorous and detailed ethnography, to analysis of discussions on internet fora, and surveys). Traditionally, philosophy is concerned with introspective conceptual analysis concerning a range of issues such as knowledge, morality, values, mind, and language. Recently, the idea that, to study these concepts requires a closer look at how they play out in real-life contexts gains traction, which has led to the approach of 'empirical philosophy.' However, the relation of philosophical, conceptual analysis to empirical material is still widely debated among philosophers engaged in empirical work, and many questions are still unanswered. Examples of methodological questions that are open for debate in empirical philosophy are: Is philosophical analysis fundamentally different from theory formation through empirical study? Should empirical philosophy develop its own research methodology, or can it adopt methods from other disciplines? And in the latter case, how closely should empirical philosophy follow these, more established, methods?

In the philosophy of science, more specifically, a similar shift towards empirical philosophy can be identified. As mentioned earlier in this dissertation, traditional philosophy of science is mainly concerned with scientific laws and theories and how truthful they are, in the sense of how well they represent the world. In contrast to this approach, the society for philosophy of science in practice (SPSP) also concerns itself with how knowledge is generated in 'real-world' scientific research practices, i.e., what activities are required 
to generate knowledge. In their mission statement, ${ }^{51}$ one of the points of attention listed is: "it is crucial to have a productive interaction between philosophical reasoning and a study of actual scientific practices, past and present." According to scholars who have adopted the issues that the philosophy of science in practice (PSP) aims at, this requires that philosophers study (cases of) research practices in which (scientific) knowledge is generated, rather than only the outcome of these practices. In other words, not only should the scientific theories, concepts and laws as they are written in journal articles or textbooks be studied, but also the processes by which they were constructed. As a result, philosophers of science in practice deal with similar issues to those that I have mentioned above. Within SPSP, scholars have discussions about how the empirical data needed for philosophical conclusions can best be obtained, how it should be handled and what type of philosophical conclusions can be derived from this data. In the following paragraph, I will aim to provide an outline of how I have dealt with these types of questions in my research.

This dissertation is a contribution to the philosophy of science in practice (PSP) project and also aims to contribute to its discussions about method. I have turned my attention to practices and knowers in order to gain a well-informed conceptual understanding of how knowledge is generated in these practices, which is one of the central assumptions on method in PSP. By developing a vocabulary of philosophical concepts to understand the practices that I have studied, I have developed a conceptual framework.

This conceptual framework should allow practitioners to obtain a deeper understanding of their own epistemic practices and activities and provide guidance as to how they might improve or justify them. For instance, my analysis of the concept of disciplinary perspectives might help them understand that they see a target system differently from their co-worker who has a different disciplinary perspective, which might help to develop interdisciplinary solutions (see Chapter 3 for a more detailed discussion). Providing a conceptual analysis and introducing new concepts to describe this practice also allows practitioners to identify and evaluate aspects of their practice that they were not able to see or think about before. ${ }^{52}$ Practitioners are thereby enabled to further reason and gain new insight about their practice, which then leads to further development and adaptation of the conceptual framework.

51 See: http://www.philosophy-science-practice.org/about/mission-statement

52 This idea has been introduced by Joe Rouse (2011). Articulating the world: Experimental systems and conceptual understanding. International Studies in the Philosophy of Science, 25(3), 243-254 DOI: $10.1080 / 02698595.2011 .60524610 .1080 / 02698595.2011 .605246$ 
In that sense, the conceptual framework developed in philosophy and actually used by practitioners, can be seen as similar to the development and use of an epistemic tool in, for instance, medical practices: clinicians construct and use a 'picture' of their patients that functions as an epistemic tool for further reasoning about diagnosis and treatment. As I have argued in this dissertation, regarding knowledge (or in this case, a conceptual framework) as an epistemic tool introduces other criteria for accepting that knowledge than truthfulness. More specifically, the conceptual framework should be adequate for the studied practice, which implies that the framework must be empirically adequate. Also, rather than being comprehensive or 'complete,' the comprehensiveness of the resulting conceptual framework must comply with the epistemic aim (i.e., the intended epistemic or explanatory uses) of the framework. Furthermore, the conceptual framework (or vocabulary developed in this thesis) should be sufficient, but also understandable and manageable for the epistemic users (experts in medical practice), as otherwise, it will not serve as a fruitful tool. ${ }^{53}$ Finally, an epistemic tool is open-ended and flexible. It can be adapted for use in other contexts or to fit changes or developments of that practice and new insights about it.

By interpreting the conceptual frameworks as epistemic tools rather than a (philosophical) theory, I aim to emphasize that there is no passive representational relationship between the vocabulary that I have developed in this dissertation and the practice for which it is developed. Instead, the vocabulary has been developed in an interaction that is mediated by a knower - in this case, me - who is actively involved in the construction of the vocabulary. This may raise the question whether such an approach and interpretation of the vocabulary developed in my research is ultimately very personal and subjective. Indeed, throughout my research project, I have made choices regarding the empirical data while pre-existing ideas and questions I had and my own disciplinary perspective(s) have shaped what aspects I pick out from an overabundance of data as worthwhile for further analysis. However, in accordance with interpreting the developed conceptual framework as an epistemic tool for specific purposes and practices, instead of striving for objectivity and detachment, the more important measure for the scientific quality of the framework is whether it meets criteria such as empirical adequacy, coherency, intelligibility and usefulness. In the research project, there have been many ways in which this was safeguarded and warranted. For example, most of the notions were developed

53 See Chapter 2 and Boon, M. (2017), 'An engineering paradigm in the biomedical sciences: Knowledge as epistemic tool', Progress in Biophysics and Molecular Biology, 129, 25-39 DOI: 10.1016/j.pbiomolbio.2017.04.001. For a more detailed account of knowledge as epistemic tools. 
in collaboration and interaction with others: (co-)supervisors, study subjects, reviewers and audiences at scientific conferences have commented on drafts of papers and by that had a smaller or larger impact on the analysis. In that way, my personal involvement does not necessarily imply subjectivity but makes it part of an intersubjective tradition and with that subject to certain standards and epistemic values. Additionally, as required in any scientific work, by displaying quotes, including references and providing arguments, I aim to make my line of argument and the evidence for my claims accessible to others for scrutiny. To finish, as an epistemic tool, the ultimate justification of this conceptual framework comprises its use. Therefore, I wholeheartedly invite practitioners to take up the vocabulary that I present in this dissertation and apply it to their practice, evaluate its fit to their own specific context and its fruitfulness to gain a deeper understanding of epistemic activities. It is only by this process - in which the tool will be adapted and improved - that the potential of this dissertation can be properly assessed. 


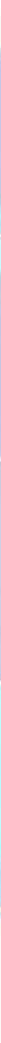




\section{Appendix}

Summary

Samenvatting

List of publications

Biography

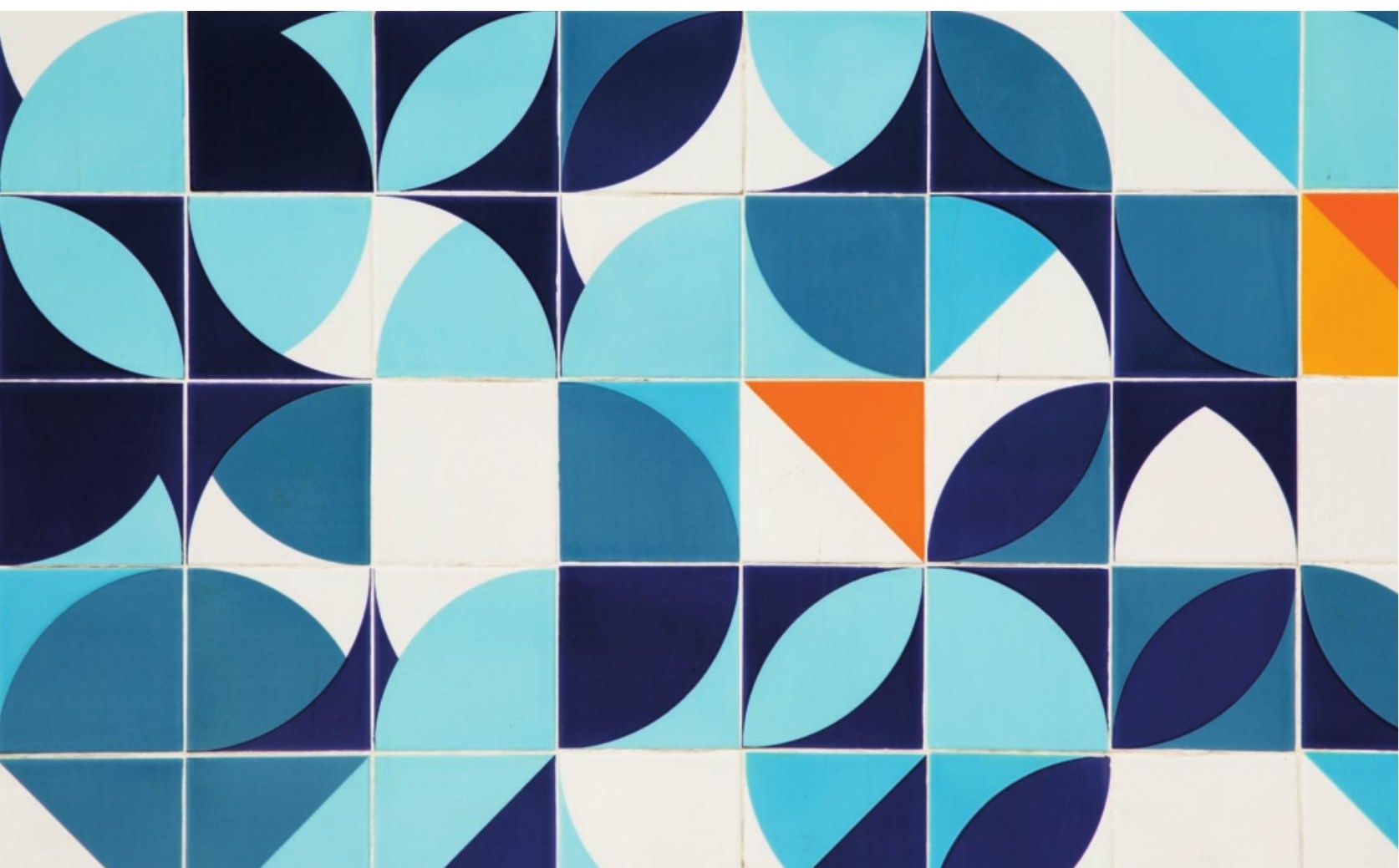




\section{Summary}

This dissertation deals with knowing in medical practice. More specifically, I have analysed different aspects of knowing in medical practice, addressing questions like: what kind of expertise is needed to use and produce knowledge; how is knowledge constructed in interdisciplinary collaborations; what role do social processes such as trust play in the construction and use of knowledge in multidisciplinary teams; how is information that is provided by new imaging technologies accepted as evidence to make diagnosis and treatment decisions; what role does knowledge play in developing imaging technologies; and, what role do imaging technologies play in the construction of knowledge for clinical decision-making. These are epistemological questions on the role of knowledge in decision-making concerning diagnosis and treatment in medical practice. In this dissertation, answering these questions diverts from traditional approaches in the philosophy of science by focusing on what people (i.e., epistemic agents such scientists, engineers, and medical professionals) do when they construct and use knowledge, and what criteria play a role in evaluating epistemic results.

I have taken a philosophy of science in practice approach, which combines a focus on the different roles that knowledge plays in medical practices with concepts from philosophy of science. This gave me the opportunity to develop an in-depth epistemological analysis of clinical decision-making, as well as an epistemological analysis of expertise, imaging technologies and interdisciplinarity in medical practice. One of my aims was to study why interdisciplinary collaborations are imperative to develop medical imaging technologies. Medical images do not speak for themselves but need to be interpreted, which requires an understanding of medical practice to establish for what relevant clinical claims (i.e., claims that impact diagnosis and treatment of individual patients) they might provide evidence, whereas an understanding of the imaging technology is required to establish the reliability (i.e., whether it is obtained in a technologically sound manner) of this evidence. Therefore, imaging developers (engineers) and users (clinicians) need to enter into a shared search process to establish what an image represents, by together establishing the relevance and reliability of the evidence an imaging tool can provide. To understand how they can effectively combine their different expertises, what makes these interdisciplinary collaborations difficult, and how epistemic difficulties with interdisciplinary research can be understood and dealt with, I have studied the development of new imaging tools for clinical practice in two ways. First, by working as a technical medicine researcher on the development of a new MRI tool, diffusion MRI to characterize kidney tumours, together with engineers, radiologists, urologists and physicists. By being involved in this research, I gained first-hand experience with what researchers do, epistemically, when they aim to 
develop a reliable and relevant imaging tool, and what epistemological issues and difficulties (for example, with interdisciplinarity) they encounter. Second, by investigating how a multidisciplinary team collaboratively analyses and interprets a new magnetic resonance imaging (MRI) tool for the diagnosis of airway diseases such as asthma and chronic obstructive pulmonary disease (COPD), called 'hyperpolarized gas MRI'. This allowed me to zoom in on the actual interdisciplinary collaborative reasoning process by which what an image represents and how it can be interpreted in the specific clinical context is established.

In addition, I studied how, in practice, medical professionals construct knowledge about their patients, using different sources of evidence such as medical history, clinical observations and medical imaging, and how professionals with different expertises collaborate in the construction of clinical knowledge. Therefore, I have performed a qualitative study of how a multidisciplinary team of medical experts involved in the diagnosis and treatment of patients with pulmonary hypertension, a rare disease, combine their expertises to come to a shared clinical decisions. This study was based on observations, interviews, video recordings and a panel discussion, and focused on analysing how these experts exchange and construct knowledge. In this multidisciplinary team, knowing was distributed over professionals with different expertises. This distributed character of knowing involves implicit trust. Trust in these practices is implicit, in the sense that trusting the expertise of others occurs while the members of a team focus on other tasks, most importantly, building up a framework of common ways of identifying and assessing evidence. It is within this intersubjective framework that trusting or mistrusting becomes meaningful in multidisciplinary clinical teams.

The result of this study is a conceptual framework, a vocabulary consisting of a cohering body of philosophical concepts (i.e., epistemological responsibility, epistemic tools, expertise, tacit knowledge, disciplinary perspectives, interdisciplinary expertise, interdisciplinary collaborative reasoning, distributed knowing and implicit trust) that clarify what scientists or medical professionals do when they construct and use knowledge, in biomedical research and medical practices that aim to develop and implement medical imaging tools. This conceptual framework should allow practitioners to obtain a deeper understanding of their own epistemic practices and activities and provide guidance as to how they might improve or justify them.

In current medical practice, the dominant paradigm for knowing in diagnosis and treatment of individual patients is evidence-based medicine (EBM). In Chapter 1, I argue that the methodology preferred by EBM and the way EBM envisions the application of scien- 
tific results (rule-based reasoning following guidelines) does not accommodate clinical decision-making because the generic information and reasoning conflicts with the goal of diagnosis and treatment of individual patients which requires integration of general and particular information. I show that EBM is based on a 'narrow view of science' and as a consequence promotes a hierarchy in which one type of scientific research (i.e., randomized controlled trials, or RCTs) is preferred and others (basic science and unsystematic observations) are devalued. EBM fails to acknowledge, for instance, that the gathering, interpretation and application of evidence require that medical professionals make judgments. By giving examples of alternative epistemologies, I aim to make plausible that good clinical decision-making involves other types of reasoning than mere algorithmic reasoning based on the outcomes of RCTs and the general rules in EBM protocols based on these outcomes.

Making justified decisions concerning diagnosis and treatment is more complex and refined than the rule-based reasoning that EBM theory promotes. It involves the gathering and assessment of many different types of information, qualitative and quantitative, general and particular, personal and detached, local and universal. Instead of dismissing professional judgment and expertise in medical decision-making as 'unscientific,' I argue that doctors have a responsibility to 1 ) gather and use relevant information and knowledge, 2) employ different types of reasoning for specific situations, and 3) make the intellectual effort to use these types of knowledge and reasoning so as to coherently fit together heterogeneous sources of information into a coherent 'picture' of a patient. This 'picture' must be constructed such that it enables further reasoning about the patient's condition, for instance, in formulating alternative hypotheses about the disease (the diagnosis), predicting which cure may work (the treatment-plan), or in explaining why a treatment causes side-effects. This responsibility of a doctor involves the combination of professional attitude and epistemological abilities, which is why I call it epistemological responsibility.

The importance of a doctor's epistemological abilities (of gathering, assessing, interpreting, integrating and using different types of knowledge and reasoning) for clinical decision-making concerning the diagnosis and treatment of individual patients, implies that the ability to deal with knowledge is an important aspect of a doctor's expertise. Therefore, in Chapter 2, I argue that a more detailed account of expertise will help to better understand the epistemological responsibility of doctors, in terms of how it can be developed and how it can be assessed. Therefore, I focus on epistemic tools and epistemic activities to analyse how knowledge and information are used in the reasoning process to reach clinical decisions. Performing activities and using tools well requires cognitive and metacognitive skills that are developed as a part of a professional's expertise. 
Following Collins and Evans (2007), I endorse a skill-based view, rather than a knowledge-based view, of expertise, but add that an important aspect of expertise is the ability to perform epistemic activities related to medical reasoning at an expert level. As a consequence, expertise does not only involve epistemic content and physical skills, but also cognitive skills of performing specific epistemic activities. According to Collins and Evans, tacit knowledge plays a pivotal role in expertise. However, they interpret tacit knowledge as inarticulate and therefore inaccessible for inquiry and evaluation by the expert themselves or others. By referring to Michael Polanyi’s $(1958,1966)$ original notion of tacit knowledge, in which knowing comprises to types of awareness, subsidiary and focal awareness, I argue that knowledge in one's subsidiary awareness can be opened up to scrutiny. This implies that doctors can be held accountable for their tacit uses of information, be it textbook knowledge, outcomes from RCTs or experience. Although during the act of knowing it is impossible to articulate which particulars make up the background clues of the subsidiary awareness, it is very well possible to make a reconstruction of this background and to explain how it justifies certain decisions.

In my account, the gathering and critical assessment of relevant information, as well as the construction of a coherent 'picture' of the individual patient from these heterogeneous pieces of information, and also the application and adaptation of this 'picture' to the specific situation at hand, are epistemic activities that clinicians should be able to perform skillfully. Focusing on the character of these epistemic activities allows understanding what medical expertise entails, and what role tacit knowledge plays in these activities. An important result of elucidating the role of tacit knowledge in expertise is that it clarifies why and how medical experts can be held accountable for epistemic activities in medical decision-making. Therefore, I argue that cognitive skills and the competence to perform epistemic activities are crucial to medical expertise, and additionally, that doctors bear the epistemological responsibility to develop, acquire and cultivate these skills and competence similar to, and as part of, their professional responsibility to develop, acquire, and cultivate physical skills such as surgical skills. EBM was introduced as an attempt to improve the quality of clinical decision-making by making it more objective. My analysis has shown, however, that EBM is based on flawed ideas about how knowledge is constructed and used and that it does not do justice to the crucial role of experts in assessing and judging which information is relevant, how to integrated it with other sources of information, and what can be concluded from it. I have argued that the laudable intensions of EBM proponents to avoid certain forms of subjectivity cannot be realized by holding on to objectivity as the main epistemic criterion, and that the notion of epistemological responsibility of doctors is better suited to evaluate the quality of decision-making. 
After having analysing knowing in medical practice by individual experts, in Chapters 1 and 2, I turn to epistemological aspects of interdisciplinary collaborations in medical practice (Chapter 3). The ability to participate in interdisciplinary projects to develop and implement technology for diagnosis and treatment in medical practice requires what I have called interdisciplinary expertise. I develop the notion of interdisciplinary expertise by presenting a case of an interdisciplinary research project aimed at developing a new imaging tool for the characterisation of kidney tumours. Expertise is usually acquired within a certain discipline and I argue that as a result experts have a disciplinary perspective that shapes what aspects of a target-system they will 'see' or 'filter-out,' and how they will approach research concerning the target system. I view disciplinary perspectives as coherent sets of aspects such as goals, phenomena of interest, objects of investigation, ways of modelling, theories and concepts, methodology and instruments, practical constraints, epistemic values and scientific ideals. By means of presenting the case of developing an imaging technology, and pointing out the different disciplinary perspectives that are involved (i.e., MRI physics, diffusion MRI, signal and image processing, medical biology, and clinical practice concerning patients with kidney tumors), I show that by having different disciplinary perspectives, each expert can focus on different aspects of the target system and perform different epistemic activities, hence making different contributions to the common goal.

Looking at the interdisciplinary teamwork in detail, I show that these experts move from one disciplinary perspective to another when discussing aspects of the target system. I defend that recognizing the disciplinary perspectives as cognitive frameworks within which each expert reasons and approaches the target system, helps in better understanding each other. What makes a research project interdisciplinary is that multiple disciplinary perspectives contribute to a problem solution that is internally coherent as well as consistent with relevant aspects of the contributing disciplines. I argue for a 'problem-oriented' account of interdisciplinarity, were disciplinary perspectives interact around a problem concerning a specific target system. Rather than viewing interdisciplinary research as aiming at integration of theories and concepts of the disciplines, interdisciplinary research in a problem-oriented approach involves that multiple disciplinary perspectives contribute to a problem solution that is internally coherent as well as consistent with relevant aspects of the contributing disciplines.

In addition to disciplinary expertise, experts involved in interdisciplinary research projects need to have interdisciplinary expertise which, in my view, crucially includes the ability to adequately deal with disciplinary perspectives. I argue that this involves cognitive skills at two levels: the first level allows the use of multiple disciplinary perspective 
to reason about a target system, whereas the second level allows reflecting on multiple disciplinary perspectives to their differences and similarities in order to identify evaluate where gaps, opportunities and conflicts might arise and how this can be resolved into a solution that is at least acceptable to each discipline involved. Finally, I turn to technical medicine, an educational program that aims to train interdisciplinary professionals. I argue that technical physicians have a well-developed form of interdisciplinary expertise. They are specialized in solving interdisciplinary problems (i.e., medical problems that require technological solutions), and are familiar with the disciplines involved to such an extent that they can take the role of 'connector,' facilitating profound interactions between and contributions by disciplinary experts (e.g., clinicians and engineers), which may not be successful if left to disciplinary experts themselves.

In Chapter 4 I investigate how a multidisciplinary research team, consisting of a clinician, a radiologist, a radiographer and an MRI engineer, collaboratively analyses and interprets a new MRI tool for the diagnosis of airway diseases, called 'hyperpolarized gas MRI,' that has not been adopted into daily clinical practice yet. This allowed me to zoom in on the actual reasoning processes and point out why epistemic exchange between different experts during the development of the imaging technology are imperative for the successful development of a clinical imaging tool. I argue that the success of developing a new imaging tool for clinical practice hinges on the reliability, relevance, usefulness, and interpretability of the imaging tool for the intended use (e.g., diagnosis). Therefore, future users, i.e., the clinicians, have to be involved by making empirical contributions in this type of research practices. Establishing the representation and interpretation of an image results from a collaborative search process. In this search process, team members enter into multiple cycles of abduction - deduction - induction, in the sense of C.S. Peirce, and team members coordinate and adapt their interpretations and contributions in such a way that they feed to each other. Suggestions and questions from the clinician help the engineer to articulate follow-up research, whereas the interpretations by the radiologists help the clinician to better understand his patient's condition. This brings forth an interdisciplinary collaborative reasoning process. In my analysis, I focus on the collaborative interdisciplinary reasoning processes to illustrate and support these claim.

Establishing how and imaging technique represents from a technological point of view, and what it represents from a clinical point of view involves interpretation of the workings of the technology and of the raw data, from the respective disciplinary perspectives, in the context of a specific patient population. Therefore, how and what the imaging technology represents is interdependent and therefore needs to be established in an interdisciplinary collaboration. what and how it represents, and its interpretation (in the context of a 
specific patient population) are interdependent and therefore need to be established side by side. In an iterative process, clinicians come to relate aspects of the image to aspects of their patients, while developers come to understand which features of the image are valuable to clinicians and subsequently tweak the technology so that these are highlighted amongst the abundance of other data. It is through the interaction of the technology, the image, and the experts' input that the representation and interpretation of an image become established.

In Chapter 5 I argue that knowing in current medical practice has an inherently social character and that imaging plays a mediating role in these practices in three ways: firstly as enablers or tools in acquiring information; secondly as communication facilitators, and thirdly as pervasively framing the epistemic domain by setting standards by which novel imaging tools are assessed. I have analysed clinical decision-making within a medical expert team involved in diagnosis and treatment of patients with pulmonary hypertension $(\mathrm{PH})$, a rare disease requiring multidisciplinary team involvement. Decision-making in the $\mathrm{PH}$ clinic involves combining evidence from heterogeneous sources generated by people with different expertises into a coherent 'picture' of a patient. In this socially distributed process, each expert needs to craft a coherent 'picture' of a patient that suits to their own discipline, which must also be consistent with relevant insights and information provided by experts from the other disciplines. It is in this way that the knowledge of a patient (consisting of different 'pictures' developed by disciplinary experts) is socially distributed. Multidisciplinary team meetings are an important place where experts interact and share, discuss, interpret and adjust information, which allows them to develop a collective way of 'seeing' and a shared language to communicate about it.

In Chapter 6 I argue that the distributed knowing character of clinical decision-making raises important questions for the epistemology of medicine, which is inherently social in this kind of setting and implies epistemic dependence on others. Trust in these contexts is a highly complex social practice, involving different forms of relationships between trust and reasons for trust: based on reasons that are easily accessible to reflection and others that are not. I focus on what it means to have reasons to trust colleagues in an established clinical team, who collectively carry out every day clinical decision-making. I show two important points about these reasons, firstly, reasons for trust are not sought or given in advance of a situation of epistemic dependence, but are established within these situations; secondly, that these reasons are implicit in the sense of being contained or nested within other actions that are not directly about trusting another person. The processes of establishing these reasons are directly about accomplishing a task, and indirectly about trusting someone else's expertise or competence. These processes establish an 
inter-subjective framework within which what it means to have reasons for trust, or not, gains a meaning and traction in these team-work settings. Based on a qualitative study of decision-making in image-assisted diagnosis and treatment of pulmonary hypertension $(\mathrm{PH})$, I show how an intersubjective framework is established through team members forging together a common way of identifying and dealing with evidence. In dealing with images as a central diagnostic tool, this also involves a common way of looking at the images, a common mode or style of perception. These frameworks are developed through many iterations of adjusting and calibrating interpretations in relation to those of other members of the multidisciplinary team, thereby establishing what counts as evidence and how to rank different kinds of evidence. Implicit trust is at work throughout this process. Trusting the expertise of others in clinical decision-making teams occurs in the process of building up a framework of common modes of seeing, and in establishing common ways of identifying and assessing evidence. It is only in this way, that trusting or mistrusting becomes meaningful, and that a framework for epistemic dependence is established. 


\section{Weten in de Medische Praktijk}

Expertise, Beeldvormingstechnologieën en Interdisciplinariteit

\section{Samenvatting}

Dit proefschrift gaat over weten in de medische praktijk. Om precies te zijn, ik heb verschillende aspecten van weten in de medische praktijk geanalyseerd, waarbij ik me heb bezig gehouden met vragen als: wat voor expertise is nodig om kennis te gebruiken en te produceren; hoe wordt kennis geconstrueerd in interdisciplinaire samenwerkingen; welke rol spelen sociale processen, zoals vertrouwen, in de constructie en het gebruik van kennis in multidisciplinaire teams; hoe wordt informatie die is verkregen met behulp van beeldvormingstechnologieën gebruikt als bewijs voor behandel- en diagnosebeslissingen; welke rol speelt kennis bij de ontwikkeling van beeldvormingstechnologieën; en, welke rol spelen beeldvormingstechnologieën in de constructie van kennis voor klinische besluitvorming. Dit zijn epistemologische vragen over de rol van kennis in de medische besluitvormingspraktijk. Voor het beantwoorden van deze vragen wijk ik in dit proefschrift af van traditionele benaderingen in de wetenschapsfilosofie door me te richten op wat verschillende mensen (namelijk, wetenschappers, ingenieurs en medische professionals) doen wanneer zij kennis construeren en gebruiken, en welke criteria een rol spelen bij het evalueren van epistemische resultaten.

De benadering in dit proefschrift is die van filosofie van wetenschap in praktijk. Deze benadering combineert een focus op de verschillende rollen die kennis speelt in medische praktijken met concepten uit de wetenschapsfilosofie. Hiermee kon ik een diepgaande epistemologische analyse van klinische besluitvorming ontwikkelen, evenals een epistemologische analyse van expertise, beeldvormingstechnieken en interdisciplinariteit in de medische praktijk. Een van mijn doelen was om te onderzoeken waarom interdisciplinaire samenwerkingen noodzakelijk zijn om medische beeldvormingstechnologieën te ontwikkelen. Medische beeldvorming spreekt niet voor zichzelf, maar moet worden geïnterpreteerd. Dit vereist een goed begrip van de medische praktijk om vast te kunnen stellen voor welke relevante klinische claims (claims die van invloed zijn op de diagnose en behandeling van individuele patiënten) beelden bewijs kunnen leveren, terwijl een goed begrip van de beeldvormingstechnologie is vereist om de betrouwbaarheid van dit bewijs vast te stellen (of het op een technologisch verantwoorde manier wordt verkregen). Daarom moeten ontwikkelaars (ingenieurs) en gebruikers (clinici) een gedeeld zoekproces aangaan om vast te stellen wat een beeld representeert. Samen stellen zij de relevantie en betrouwbaarheid vast van het bewijs dat een beeldvormingsinstrument kan bieden. Om te begrijpen hoe ze hun verschillende expertises effectief kunnen combineren, wat deze interdisciplinaire samenwerkingen moeilijk maakt en hoe epistemologische 
problemen in interdisciplinair onderzoek kunnen worden begrepen en aangepakt, heb ik de ontwikkeling van nieuwe beeldvormingsinstrumenten voor klinische praktijken op twee manieren bestudeerd. Ten eerste, door als technische geneeskundig onderzoeker samen met ingenieurs, radiologen, urologen en natuurkundigen te werken aan de ontwikkeling van een nieuwe MRI-tool, genaamd diffusie-MRI, voor de karakterisering van niertumoren. Door betrokken te zijn bij dit onderzoek heb ik uit de eerste hand ervaring opgedaan met wat onderzoekers doen, epistemologisch, wanneer ze een betrouwbaar en relevant beeldvormingsinstrument willen ontwikkelen en welke epistemologische problemen en moeilijkheden ze tegenkomen. Ten tweede door te onderzoeken hoe een multidisciplinair team een nieuwe MRI-tool analyseert en interpreteert voor de diagnose van luchtwegaandoeningen zoals astma en chronische obstructieve longziekte (COPD), genaamd 'hyper gepolariseerd gas-MRI'. Hierdoor kon ik inzoomen op het feitelijke interdisciplinaire collaboratieve redeneerproces waarmee wordt vastgesteld wat een beeld representeert en hoe het kan worden geïnterpreteerd in de specifieke klinische context.

Daarnaast heb ik onderzocht hoe, in de praktijk, medische professionals kennis over hun patiënten construeren, met behulp van verschillende bronnen van bewijs, zoals uit anamnese, klinische observaties en medische beeldvorming, en hoe professionals met verschillende expertises samenwerken in de constructie van klinische kennis. Daarvoor heb ik een kwalitatief onderzoek uitgevoerd naar hoe een multidisciplinair team van medische experts die betrokken zijn bij de diagnose en behandeling van patiënten met pulmonale hypertensie, een zeldzame ziekte, hun expertise combineert om tot een gedeelde klinische beslissing te komen. Deze studie was gebaseerd op observaties, interviews, video-opnamen en een paneldiscussie en was gericht op het analyseren van hoe deze experts kennis uitwisselen en construeren. In dit multidisciplinaire team werd weten gedistribueerd over professionals met verschillende expertises. Dit gedistribueerde karakter van weten vereist impliciet vertrouwen. Vertrouwen in deze praktijken is impliciet, in de zin dat vertrouwen op de deskundigheid van anderen plaatsvindt terwijl de leden van een team zich concentreren op andere taken, met name het opbouwen van een raamwerk van gemeenschappelijke manieren om bewijs te herkennen en te beoordelen. Het is binnen dit intersubjectieve kader dat vertrouwen of wantrouwen zinvol wordt in multidisciplinaire klinische teams.

Het resultaat van deze studie is een conceptueel kader, een vocabulaire bestaande uit een samenhangend geheel van filosofische concepten (epistemologische verantwoordelijkheid, epistemisch gereedschap ('epistemic tools'), expertise, impliciete kennis ('tacit knowledge'), disciplinaire perspectieven, interdisciplinaire expertise, interdisciplinair collaboratief redeneren, gedistribueerd weten en impliciet vertrouwen) die verduidelijken wat wetenschappers of medische professionals doen wanneer ze kennis construeren en gebruiken, in de medische 
praktijk en in de onderzoekspraktijk waarin medische beeldvormingstechnieken worden ontwikkeld. Dit conceptuele kader moet beoefenaars in staat stellen een dieper inzicht te verwerven in hun eigen epistemologische praktijken en activiteiten en handvatten bieden voor hoe ze deze kunnen verbeteren of rechtvaardigen.

In de huidige medische praktijk is evidence-based medicine (EBM) het dominante paradigma voor kennisvorming in diagnose en behandeling van individuele patiënten. In Hoofdstuk 1 beargumenteer ik dat EBM geen rekening houdt met klinische besluitvorming in de praktijk, omdat de door EBM geprefereerde methodologie en manier van toepassen van wetenschappelijke resultaten (algoritmisch redeneren volgens richtlijnen) in strijd zijn met het doel, namelijk diagnose en behandeling van individuele patiënten. Hiervoor is integratie van generieke en (patiënt-)specifieke informatie vereist. Ik laat zien dat EBM gebaseerd is op een 'nauwe visie op de wetenschap' en bijgevolg een hiërarchie promoot waarin een bepaald type wetenschappelijk onderzoek (gerandomiseerde gecontroleerde studies, of RCT's) de voorkeur heeft boven basale wetenschap en niet-systematische waarnemingen. EBM erkent bijvoorbeeld niet dat het verzamelen, interpreteren en toepassen van bewijsmateriaal vereist dat medische professionals een oordeel vormen. Aan de hand van voorbeelden van alternatieve epistemologieën probeer ik aannemelijk te maken dat goede klinische besluitvorming ander redeneerstijlen vereist dan louter algoritmisch redeneren op basis van de uitkomsten van RCT's en de algemene regels in EBM-protocollen op basis van deze uitkomsten.

Het maken van gerechtvaardigde beslissingen met betrekking tot diagnose en behandeling is complexer en verfijnder dan de op regels gebaseerde redenering die de EBM-theorie promoot. Het omvat het verzamelen en beoordelen van veel verschillende soorten informatie, kwalitatief en kwantitatief, generiek en specifiek, persoonlijk en onpersoonlijk, lokaal en universeel. In plaats van professionele oordeelsvorming en deskundigheid in medische besluitvorming als 'onwetenschappelijk' af te wijzen, pleit ik ervoor dat artsen een verantwoordelijkheid hebben om 1) relevante informatie en kennis te verzamelen en te gebruiken, 2) verschillende soorten redeneerstijlen te hanteren voor specifieke situaties, en 3) de intellectuele inspanning te leveren om dit soort kennis en redeneerstijlen te gebruiken om heterogene informatiebronnen op coherente wijze samen te brengen tot een samenhangend 'beeld' van een patiënt. Dit 'beeld' moet zodanig zijn geconstrueerd dat deze het mogelijk maakt om verder te redeneren over de conditie van de patiënt, bijvoorbeeld door het formuleren van alternatieve hypothesen over de ziekte (de diagnose), het voorspellen welke behandeling kan werken (het behandelplan), of het uitleggen waarom een behandeling bijwerkingen veroorzaakt. Deze verantwoordelijkheid van een arts omvat de combinatie van professionele houding en epistemologische vaardigheden, en daarom noem ik deze verantwoordelijkheid epistemologische verantwoordelijkheid. 
Het belang van de epistemologische vaardigheden van een arts (voor het verzamelen, beoordelen, interpreteren, integreren en gebruiken van verschillende soorten kennis en redeneerstijlen) voor klinische besluitvorming over de diagnose en behandeling van individuele patiënten impliceert dat het vermogen om met kennis om te gaan een belangrijk aspect is van de expertise van een arts. Daarom beargumenteer ik in Hoofdstuk 2 dat een preciezere invulling van expertise zal helpen om de epistemologische verantwoordelijkheid van artsen beter te begrijpen, in termen van hoe deze kan worden ontwikkeld en hoe deze kan worden beoordeeld. Daarom concentreer ik me op epistemisch gereedschap ('epistemic tools') en epistemische activiteiten om te analyseren hoe kennis en informatie worden gebruikt in het redeneerproces om tot klinische beslissingen te komen. Voor het uitvoeren van activiteiten en het gebruik van gereedschap zijn vaardigheden nodig die worden ontwikkeld als onderdeel van de expertise van een professional.

In navolging van Collins en Evans (2007), onderschrijf ik een op vaardigheden gebaseerd begrip in plaats van een op kennis gebaseerd begrip van expertise, maar ik voeg toe dat een belangrijk aspect van expertise het vermogen is om epistemische activiteiten met betrekking tot medisch redeneren uit te voeren op een expertniveau. Als gevolg hiervan omvat expertise niet alleen epistemische inhoud en fysieke vaardigheden, maar ook cognitieve vaardigheden voor het uitvoeren van specifieke epistemische activiteiten. Volgens Collins en Evans speelt impliciete kennis ('tacit knowledge') een cruciale rol in expertise. Ze interpreteren impliciete kennis echter als onuitspreekbaar en daarom ontoegankelijk voor onderzoek en evaluatie, zowel door de expert zelf als door anderen. Door te verwijzen naar Michael Polanyi's (1958, 1966) oorspronkelijke notie van impliciete kennis laat ik zien dat er een onderscheid is tussen ondersteunende en focale bewustzijn ('subsidiary' en 'focal awareness'), en dat kennis in iemands ondersteunend bewustzijn nader kan worden onderzocht om deze te evalueren. Hiermee kunnen artsen verantwoordelijk worden gehouden voor welke informatie impliciet wordt gebruikt, of het nu kennis uit leerboeken is, resultaten uit medisch-wetenschappelijk onderzoek of op basis van ervaring. Ook al is het in de activiteit van het toepassen van kennis niet mogelijk is om te verwoorden welke specifieke informatie wordt gebruikt in het ondersteunend bewustzijn, achteraf kan hier een reconstructie van worden gemaakt.

De epistemische activiteiten die clinici vaardig moeten kunnen uitvoeren zijn het verzamelen en kritisch beoordelen van relevante informatie, de constructie van een coherent 'beeld' van de individuele patiënt uit deze heterogene stukjes informatie en de toepassing en aanpassing van dit 'beeld' aan de specifieke situatie. Aan de hand hiervan is het mogelijk om te begrijpen wat medische expertise inhoudt, en welke rol impliciete kennis speelt in deze activiteiten. Een belangrijk resultaat van het ophelderen van de rol van impliciete kennis in expertise is dat het duidelijk maakt hoe medische experts verantwoordelijk kunnen worden gehouden 
voor die epistemische activiteiten die cruciaal zijn voor medische expertise. Artsen hebben een verantwoordelijkheid om hun bekwaamheid in deze activiteiten op dezelfde manier te ontwikkelen en onderhouden net als dat ze een verantwoordelijkheid hebben voor het ontwikkelen, en onderhouden van fysieke vaardigheden zoals chirurgische vaardigheden.

EBM is geïntroduceerd om de kwaliteit van klinische besluitvorming te verbeteren door dit proces objectiever te maken. Mijn analyse laat echter zien dat EBM gebaseerd is op gebrekkige ideeën over hoe kennis wordt opgebouwd en gebruikt, waarbij geen oog is voor de rol van experts in het beoordelen van de relevantie van informatie, hoe deze kan worden geïntegreerd met andere informatiebronnen en wat er uit kan worden geconcludeerd. Ik heb betoogd dat de lovenswaardige intenties van EBM-voorstanders om bepaalde vormen van subjectiviteit te vermijden niet kunnen worden gerealiseerd door vast te houden aan een specifieke interpretatie van objectiviteit als het belangrijkste epistemische criterium, en dat het begrip epistemologische verantwoordelijkheid van artsen beter geschikt is om de kwaliteit van besluitvorming te beoordelen.

$\mathrm{Na}$ het analyseren van kennis in de medische praktijk door individuele deskundigen, richt ik me in Hoofdstuk 3 op epistemologische aspecten van interdisciplinaire samenwerkingen in de medische praktijk. Het vermogen om deel te nemen aan interdisciplinaire projecten om beeldvormingstechnologieën te ontwikkelen voor diagnose en behandeling in de medische praktijk vereist wat ik interdisciplinaire expertise noem. Ik beschrijf een interdisciplinair onderzoeksproject gericht op het ontwikkelen van een nieuwe beeldvormingsmethode voor de karakterisering van niertumoren om het concept van interdisciplinaire expertise te ontwikkelen. Expertise wordt meestal binnen een bepaalde discipline verworven en ik beargumenteer dat deskundigen daardoor een disciplinair perspectief hebben dat bepaalt welke aspecten van een doelsysteem ze zullen 'zien' of 'uitfilteren'. Ik beschouw een disciplinaire perspectief als een samenhangende set van onderzoeksdoel, fenomenen, onderzoeksobjecten, manieren van modelleren, theorieën en concepten, methodologie en instrumentatie, praktische beperkingen, epistemische waarden en wetenschappelijke idealen. Door een analyse van de verschillende disciplinaire perspectieven die bij het onderzoeksproject betrokken waren (MRI-fysica, diffusie MRI, signaal- en beeldverwerking, medische biologie en de klinische praktijk met betrekking tot patiënten met niertumoren) laat ik zien dat elke expert zich kan concentreren op verschillende aspecten van het doelsysteem en verschillende epistemische activiteiten kunnen uitvoeren doordat zij verschillende disciplinaire perspectieven heeft. Op die manier kan elke expert verschillende bijdragen aan het gemeenschappelijke doel leveren. 
Met een gedetailleerde beschrijving van het interdisciplinaire teamwerk laat ik zien dat deze experts afwisselend verschillende disciplinaire perspectieven aannemen wanneer ze aspecten van het doelsysteem bespreken. Ik verdedig dat het erkennen van disciplinaire perspectieven als cognitieve kaders waarbinnen elke expert redeneert en het doelsysteem benadert helpt om elkaar beter te begrijpen. Daarbij pleit ik voor een 'probleemgerichte' benadering van interdisciplinariteit, waarbij experts vanuit hun eigen specifieke disciplinaire perspectieven een interactie aangaan rond een probleem met een specifiek doelsysteem. In plaats van interdisciplinair onderzoek te beschouwen als gericht op integratie van theorieën en concepten van de disciplines, impliceert een probleemgerichte benadering van interdisciplinair onderzoek dat meerdere disciplinaire perspectieven bijdragen aan een probleemoplossing die intern coherent is en consistent is met relevante aspecten van de bijdragende disciplines .

Naast disciplinaire expertise moeten experts die betrokken zijn bij interdisciplinaire onderzoeksprojecten interdisciplinaire expertise hebben. Interdisciplinaire expertise omvat, naar mijn mening, met name het vermogen om adequaat om te gaan met disciplinaire perspectieven. Dit impliceert dat experts cognitieve vaardigheden hebben op twee niveaus: het eerste niveau maakt het gebruik van meerdere disciplinaire perspectieven om over een doelsysteem te redeneren mogelijk, terwijl het tweede niveau reflectie op meerdere disciplinaire perspectieven omvat. Door te reflecteren kunnen verschillen en overeenkomsten tussen disciplinaire perspectieven worden geïdentificeerd om daarmee tot een oplossing te komen die niet conflicteert met de betrokken disciplines. Tot slot verwijs ik naar technische geneeskunde, een universitaire studie die interdisciplinaire professionals opleidt. Ik beweer dat technische geneeskundigen een goed ontwikkelde vorm van interdisciplinaire expertise hebben. Ze zijn gespecialiseerd in het oplossen van interdisciplinaire problemen (dat wil zeggen medische problemen waarvoor technologische oplossingen nodig zijn). Daarbij zijn ze voldoende bekend met de betrokken disciplines om de rol van 'connector' kunnen vervullen. Als connector faciliteren technisch geneeskundigen diepgaande interacties tussen de disciplinaire experts zoals clinici en ingenieurs.

In Hoofdstuk 4 onderzoek ik hoe een multidisciplinair onderzoeksteam, bestaande uit een clinicus, een radioloog, een radiologisch laborant en een MRI-ingenieur, een nieuwe MRItool voor de diagnose van luchtwegaandoeningen analyseert en interpreteert, genaamd 'hyper gepolariseerd gas-MRI.' Deze beeldvormingstechnologie wordt nog niet gebruikt in de dagelijkse klinische praktijk. Deze casus stelde mij in staat om in te zoomen op de feitelijke redeneerprocessen en aan te geven waarom epistemologische uitwisselingen tussen verschillende experts tijdens de ontwikkeling van een medische beeldvormingstechnologie noodzakelijk zijn voor de succesvolle ontwikkeling. Ik beargumenteer dat het succes van het ontwikkelen van een nieuwe imaging-tool voor de klinische praktijk afhankelijk is van 
de betrouwbaarheid, relevantie, bruikbaarheid en interpreteerbaarheid van de beeldvormende techniek voor de beoogde toepassingen (bijvoorbeeld diagnose). Daarom moeten toekomstige gebruikers, de clinici, worden betrokken bij dit soort onderzoekspraktijken door in een vroeg stadium een relatie te leggen met de toepassing in de klinische praktijk. Vaststellen wat beeldvorming representeert en hoe dit kan worden geïnterpreteerd is het resultaat van een gezamenlijk zoekproces. In dit zoekproces maken teamleden meerdere cycli van abductie - deductie - inductie, in de betekenis van C.S. Peirce, door. Suggesties en vragen van de clinicus helpen de ingenieur om vervolgonderzoek te articuleren, terwijl de interpretaties door de radiologen de clinicus helpen de toestand van zijn patiënt beter te begrijpen. Dit levert een interdisciplinair collaboratief redeneerproces op. In mijn analyse richt ik me op de interdisciplinaire collaboratieve redeneerprocessen om deze bewering te illustreren en te ondersteunen.

De beeldvormingstechniek, wat en hoe deze representeert en de interpretatie ervan (in de context van een specifieke patiëntenpopulatie) zijn onderling afhankelijk en moeten daarom tegelijkertijd worden vastgesteld. In een iteratief proces kunnen clinici aspecten van het beeld relateren aan aspecten van hun patiënten, terwijl ontwikkelaars begrijpen welke kenmerken van het beeld waardevol zijn voor clinici en vervolgens de technologie zodanig aanpassen dat deze worden benadrukt in de overvloed aan andere gegevens. Het is door de interactie van de technologie, het beeld en de input van de experts dat de representatie en interpretatie van een beeld worden vastgesteld.

In Hoofdstuk 5 beargumenteer ik dat in de huidige medische praktijk klinische besluitvorming een inherent sociaal gedistribueerd karakter heeft en dat beeldvorming een bemiddelende rol speelt in deze praktijken op drie manieren: ten eerste als hulpmiddelen bij het verwerven van informatie; ten tweede als communicatiefacilitator, en ten derde door het kaderen van het epistemologische domein, door normen tot stand te brengen waarmee nieuwe beeldvormingstechnieken worden beoordeeld. Ik heb de klinische besluitvorming geanalyseerd binnen een medisch expertteam dat betrokken is bij de diagnose en behandeling van patiënten met pulmonale hypertensie $(\mathrm{PH})$, een zeldzame ziekte waarvoor de betrokkenheid van een multidisciplinair team vereist is. Besluitvorming in de PH-kliniek omvat het combineren van bewijsmateriaal uit heterogene bronnen, verkregen door mensen met verschillende expertises, in een coherent 'beeld' van een patiënt. In dit sociaal gedistribueerde proces moet elke expert een samenhangend 'beeld' van een patiënt maken dat past bij zijn eigen discipline, maar dat ook consistent moet zijn met relevante inzichten en informatie die wordt verstrekt door experts uit de andere disciplines. Het is op deze manier dat de kennis van een patiënt (bestaande uit verschillende 'beelden' ontwikkeld door verschillende disciplinaire experts) sociaal gedistribueerd is. Multidisciplinaire team overleg- 
gen zijn een belangrijke plek waar experts communiceren en informatie delen, bespreken, interpreteren en aanpassen, waardoor ze een collectieve manier van 'zien' en een gedeelde taal kunnen ontwikkelen.

In Hoofdstuk 6 stel ik dat het sociaal gedistribueerde karakter van het construeren van kennis in klinische besluitvorming een epistemologische afhankelijkheid van anderen met zich meebrengt, wat vragen oproept voor de epistemologie van klinische besluitvorming. Vertrouwen in deze contexten is een zeer complexe sociale praktijk, waarin verschillende vormen van relaties tussen vertrouwen en redenen voor vertrouwen een rol spelen: vertrouwen gebaseerd op redenen die gemakkelijk toegankelijk zijn voor reflectie en andere die dat niet zijn. In dit hoofdstuk concentreer ik me op wat het inhoudt om redenen te hebben om collega's in een klinisch team dat zich dagelijks bezighoudt met gezamenlijke klinische besluitvorming te vertrouwen. Ik maak twee punten over deze redenen, ten eerste, ze worden niet gezocht of gegeven voorafgaand aan een situatie van epistemologische afhankelijkheid, maar worden gevestigd in deze situaties; ten tweede zijn ze impliciet omdat ze zijn ingesloten in andere activiteiten die niet direct te maken hebben met vertrouwen in iemand anders. De processen waarin deze redenen worden vastgesteld gaan direct over het uitvoeren van een taak en indirect over het vertrouwen in de deskundigheid of competentie van iemand anders. Deze processen vormen een intersubjectief kader waarbinnen wat het betekent om redenen voor vertrouwen te hebben, of niet, een betekenis krijgen in de context van het teamwerk. Op basis van een kwalitatieve studie van de besluitvorming aangaande de diagnose en behandeling van PH met behulp van medische beeldvorming, laat ik zien hoe een intersubjectief kader tot stand wordt gebracht doordat teamleden gemeenschappelijke een manier om bewijs te identificeren en te gebruiken bepalen. Bij het omgaan met klinische beeldvorming als diagnostisch instrument houdt dit ook in dat een gezamenlijke manier om beelden te gebruiken, een gemeenschappelijke perceptiestijl, wordt vastgesteld. De ontwikkeling van deze kaders doorloopt vele iteraties waarin interpretaties worden aangepast en gekalibreerd in relatie tot die van anderen, waarin wordt bepaald wat geldt als bewijs en waarin verschillende soorten bewijsmateriaal worden gerangschikt. Impliciet vertrouwen is aan het werk tijdens dit proces. Vertrouwen in de expertise van anderen in klinische besluitvormingsteams vindt plaats terwijl de teamleden bezig zijn met andere taken. Belangrijk hiervoor is het opbouwen van gemeenschappelijke manieren van kijken, en een gezamenlijke manier om bewijs te identificeren en evalueren. Op deze manier wordt vertrouwen of wantrouwen zinvol in deze context, en wordt een kader waarbinnen teamleden epistemologisch afhankelijk van elkaar kunnen zijn vastgesteld. 


\section{List of publications}

\section{This thesis}

M. Boon, S. van Baalen and M. Groenier (forthcoming) Interdisciplinary expertise in medical practice: Challenges of using and producing knowledge in complex problem-solving. Medical Teacher. DOI: 10.1080/0142159X.2018.1544417.

S. van Baalen \& A. Carusi (2017) Implicit trust in clinical decision-making by multidisciplinary teams. Synthese. DOI: 10.1007/s11229-017-1475-z.

S. van Baalen \& M. Boon (2017) Evidence-based medicine versus expertise - knowledge, skills and epistemic actions. Chapter 2 in: Knowing and Acting in Medicine. Robyn Bluhm (ed.). Rowman \& Littlefield, 21-38. ISBN: 978-178348810.

S. van Baalen, A. Carusi, I. Sabroe, \& D. G. Kiely (2016). A social-technological epistemology of clinical decision-making as mediated by imaging. J Eval Clin Pract, 23(5), 949-958. DOI: 10.1111/jep.12637.

S. van Baalen \& M. Boon (2015). An epistemological shift: from evidence-based medicine to epistemological responsibility. J Eval Clin Pract, 21(3), 433-439. DOI: 10.1111/jep.12282.

\section{Other}

S. van Baalen, M. Froeling, M. Asselman, C. Klazen, C. Jeltes, L. van Dijk, B. Vroling, P. Dik and B. ten Haken (forthcoming) Mono, bi- and tri-exponential diffusion MRI modelling for renal solid masses and comparison with histopathological findings. Cancer imaging. DOI: 10.1186/s40644-018-0178-0.

M. Boon and S. van Baalen (forthcoming) Epistemology for interdisciplinary research Shifting philosophical paradigms of science. European Journal for Philosophy of Science. DOI: 10.1007/s13194-018-0242-4.

S. van Baalen, A. Leemans, P. Dik, M. R. Lilien, B. Ten Haken, \& M. Froeling, (2017). Intravoxel incoherent motion modeling in the kidneys: Comparison of mono-, bi-, and triexponential fit. J Magn Reson Imaging, 46(1), 228-239. DOI: 10.1002/jmri.25519. 


\section{Biography}

Sophie van Baalen was born on February $19^{\text {th }} 1988$ in Woerden and grew up in Kockengen. After graduating from secondary school (VWO, RGS Broklede in Breukelen) in 2006, she started with the bachelor program Technical Medicine at the University of Twente. During her bachelor's degree, she participated in the Honours programme. In 2009 Sophie started two Master's degrees Robotics and Imaging (Technical Medicine) and Philosophy of Science, Technology and Society, both at the University of Twente. She graduated both degrees in 2014.

As part of her master's degree for Technical Medicine, Sophie worked on studying and developing diffusion magnetic resonance imaging for the kidneys at a one-year internship at the department of Pediatric Urology at the Whilhelmina Children's Hospital in Utrecht, supervised by dr. Pieter Dik and prof. dr. ir. Bennie ten Haken. Based on her observations of decision-making in the clinic, and discussions with physicians about their work during the internship, she studied the epistemological aspects of clinical decision-making and medical imaging as her master thesis for Philosophy of Science, Technologie and Society, supervised by prof. dr. ir. Mieke Boon.

Sophie won the Twente Graduate School Award in 2014 for her PhD proposal in which she combined the topics of both her master's degrees. From 2014 until 2018 she has continued her work at the departments of Philosophy and Magnetic Detection \& Imaging at the University of Twente, supervised by prof. dr. ir. Mieke Boon and prof. dr. ir. Bennie ten Haken. During her PhD she has performed field work at the University of Sheffield, supervised by dr. Annamarie Carusi.

Sophie is currently working as a researcher at the Rathenau Instituut, Den Haag. She lives in Gouda with her husband Jori and son Kasper. 




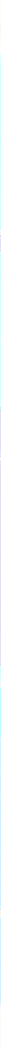

